\title{
Untersuchungen zur Energiegewinnung mit einem Solardach-Luft-Kollektor
}

\author{
Dissertation \\ zur Erlangung des Doktorgrades \\ der Fakultät für Agrarwissenschaften \\ der Georg-August-Universität Göttingen
}

vorgelegt von

Matthias Baum

geboren in Eschwege

Göttingen, im Juli 2010 
D 7

1. Referent: Prof. Dr. W. Lücke

2. Korreferent: PD Dr. E. Hessel

Tag der mündlichen Prüfung: 15. Juli 2010 


\section{Inhaltsverzeichnis}

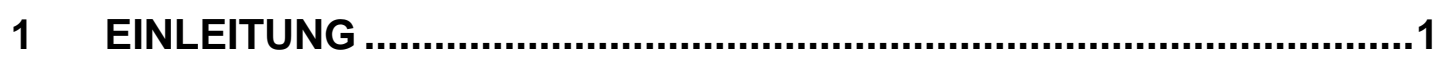

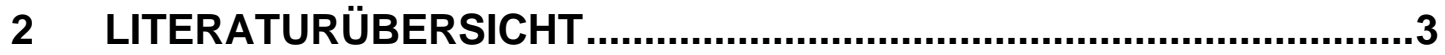

2.1 Einteilungsmöglichkeiten und besondere Eigenschaften von Solar-Luft-Kollektoren ...........................................................

2.2 Umwandlung des Strahlungsangebotes in einem Solar-

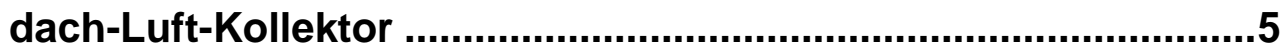

2.3 Thermische Energiegewinnung mit Solardach-Luft-

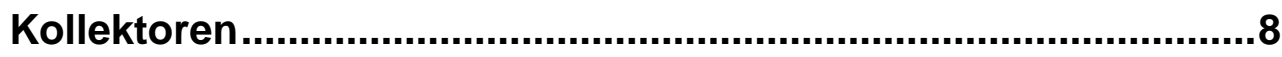

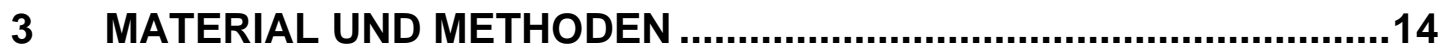

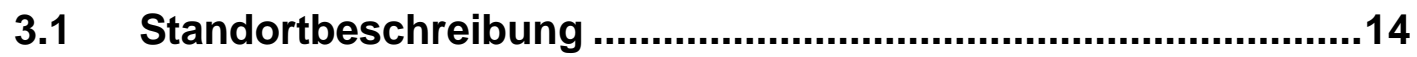

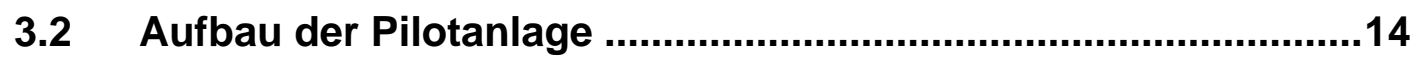

3.3 Messtechnische Gestaltung ...................................................24

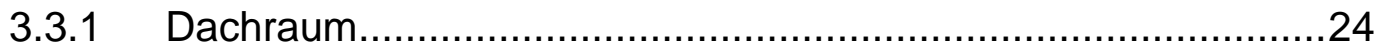

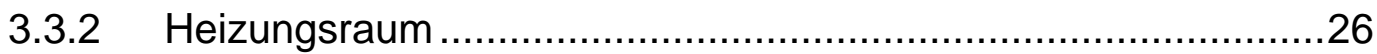

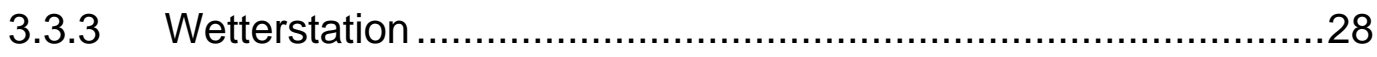

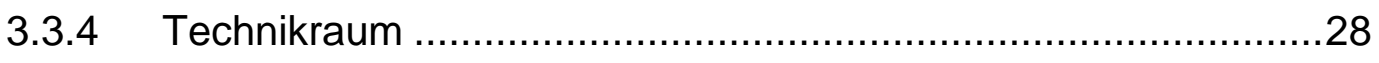

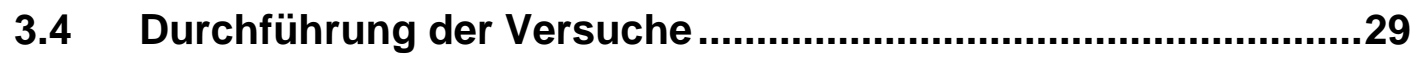

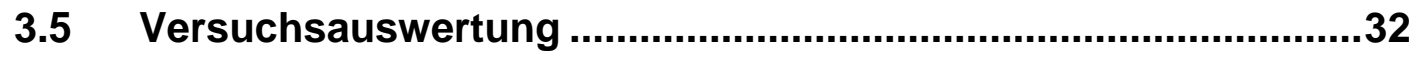


4 ERGEBNISSE .49

4.1 Solardach-Luft-Kollektor .......................................................49

4.1.1 Globalstrahlungsverlauf in Kollektorebene ..........................49

4.1.2 Temperaturerhöhung .................................................... 50

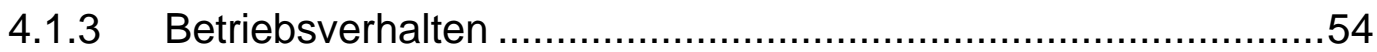

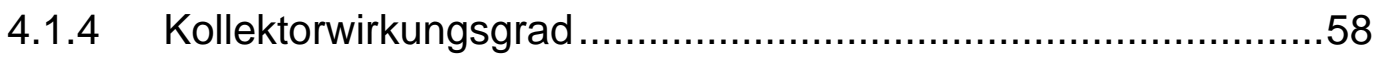

4.1.5 Einflussfaktoren auf den Luftvolumenstrom ........................61

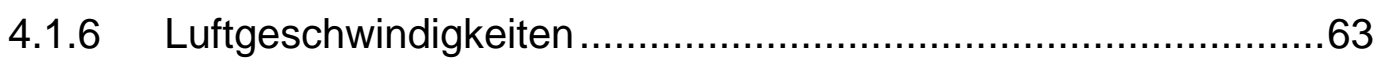

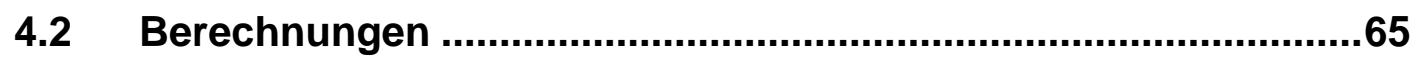

4.2.1 Energetische Betrachtung des Daches .............................65

4.2.2 Thermischer Energiebedarf der Jugendherberge...................72

4.2.3 Simulation der Wärmetauschertemperaturen .......................73

4.2.4 Simulation des Wärmepumpenbetriebes ..........................74

4.2.4.1 Betrieb bei vollständiger Wärmeabnahme ......................74

4.2.4.2 Betrieb bezogen auf die Wärmeabnahme in der Jugendherberge ....................................................... 78

4.2.4.3 Optimierung bei vollständiger Wärmeabnahme ..................83

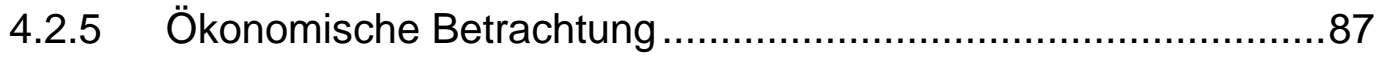

4.2.5.1 Investitionskosten der solaren Energiegewinnung ..............87

4.2.5.2 Energiebezugskosten und Amortisationszeitraum bei einer vollständigen Wärmeabnahme.

4.2.5.3 Energiebezugskosten und Amortisationszeitraum bezogen auf die Wärmeabnahme in der Jugendherberge.....

4.2.5.4 Energiebezugskosten und Amortisationszeitraum für die Optimierung bei vollständiger Wärmeabnahme .93 
5 DISKUSSION

5.1 Solardach-Luft-Kollektor ........................................................97

5.1.1 Globalstrahlungsverlauf in Kollektorebene ...........................97

5.1.2 Temperaturerhöhung und Betriebsverhalten........................98

5.1.3 Kollektorwirkungsgrad ........................................... 102

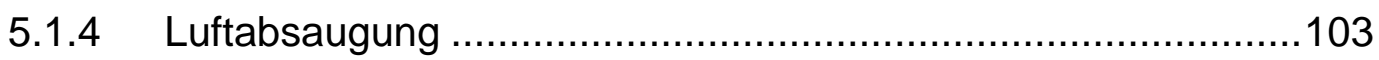

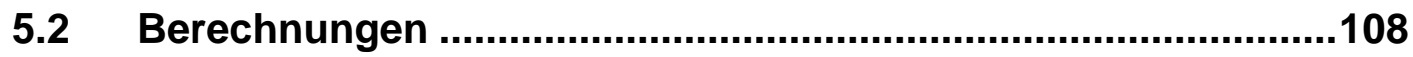

5.2.1 Energetische Betrachtung des Daches ............................108

5.2.2 Thermischer Energiebedarf der Jugendherberge..................111

5.2.3 Simulation der Wärmetauschertemperaturen ......................112

5.2.4 Simulation des Wärmepumpenbetriebes ..........................114

5.2.4.1 Vollständige Wärmeabnahme ..................................114

5.2.4.2 Wärmeabnahme in der Jugendherberge .....................118

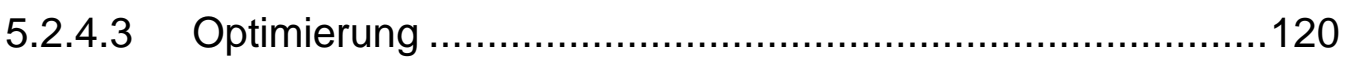

5.2.5 Ökonomische Betrachtung .......................................... 123

5.2.5.1 Investitionskosten der solaren Energiegewinnung .............123

5.2.5.2 Energiebezugskosten und Amortisationszeitraum .............124

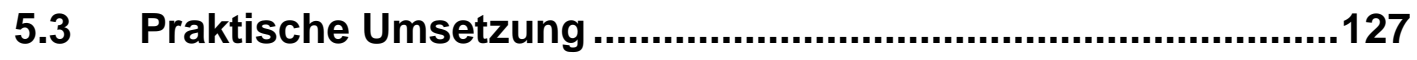

6 ZUSAMMENFASSUNG...........................................................129

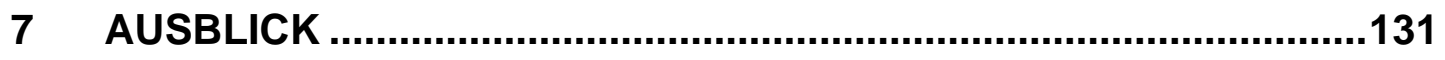

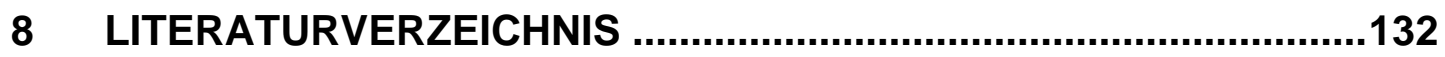




\section{Abbildungsverzeichnis}

Abbildung 1: Arten der Absorberumströmung, dargestellt am Beispiel eines abgedeckten Solar-Luft-Kollektors (Heidler et al. 2008 [40])

Abbildung 2: Schematische Darstellung der Wärmeübertragung an einem Solardach-Luft-Kollektor, verändert nach (Grimm 1984 [35])

Abbildung 3: Grundriss der Jugendherberge 15

Abbildung 4: Schematischer Aufbau eines Kollektorfeldes des Solardach-Luft-Kollektors mit Metalleindeckung .16

Abbildung 5: Schematische Darstellung der Luftabsaugung .18

Abbildung 6: Vereinfacht dargestellter Gesamtaufbau der Pilotanlage .19

Abbildung 7: Hydraulische Einbindung der Wärmepumpe mit Heißgasabschöpfung

Abbildung 8: Einbindung in den Heizungsregelungsprozess

Abbildung 9: Schematische Darstellung der Messwertaufnahme im Dachraum/Dach.

Abbildung 10: Schematische Darstellung der Messwertaufnahme im Heizungsraum.

Abbildung 11: Übersicht der Messdatenerfassung 29

Abbildung 12: Definition der Energiemengen .40

Abbildung 13: Globalstrahlungsverläufe in der Kollektorebene für ausgewählte sonnige Tage im Jahresverlauf in Abhängigkeit von der Zeit. 
Abbildung 14: Verlauf der Temperaturdifferenzen des Solardach-LuftKollektors für ausgewählte sonnige Tage im Jahresverlauf von Sonnenaufgang bis Sonnenuntergang in Abhängigkeit von der Zeit

Abbildung 15: Verlauf der Temperaturdifferenz des Solardach-LuftKollektors für einen ausgewählten sonnigen Frühlingstag (21.04.2009) in Abhängigkeit von der Globalstrahlung in Kollektorebene.

Abbildung 16: Temperaturdifferenzen des Solardach-Luft-Kollektors, dargestellt als lineare Regressionsgeraden, für ausgewählte sonnige Tage im Jahresverlauf in der ersten und zweiten Tageshälfte in Abhängigkeit von der Globalstrahlung in Kollektorebene

Abbildung 17: Temperaturdifferenzen des Solardach-Luft-Kollektors, der Dachraum- und Umgebungstemperatur mit dem Globalstrahlungsverlauf in Kollektorebene und der Spiegelung der Temperaturdifferenz der ersten Tageshälfte an einem sonnigen Frühlingstag (21.04.2009) in Abhängigkeit von der Zeit .

Abbildung 18: Temperaturdifferenzen des Solardach-Luft-Kollektors, der Dachraum- und Umgebungstemperatur mit dem Globalstrahlungsverlauf in Kollektorebene an einem wolkigen Frühlingstag (28.04.2009) in Abhängigkeit von der Zeit

Abbildung 19: Verlauf der Temperaturdifferenz des Solardach-LuftKollektors und der Globalstrahlung in Kollektorebene mit gleitenden Mittelwerten der Periode zwei an einem wolkigen Frühlingstag (28.04.2009) in Abhängigkeit von der Zeit 
Abbildung 20: Wirkungsgradverläufe des Solardach-Luft-Kollektors für ausgewählte sonnige Tage im Jahresverlauf in Abhängigkeit von der Zeit

Abbildung 21: Wirkungsgradverlauf des Solardach-Luft-Kollektors für einen ausgewählten sonnigen Tag im Frühling (21.04.2008) in Abhängigkeit von der Globalstrahlung in Kollektorebene 60

Abbildung 22: Wirkungsgradverläufe des Solardach-Luft-Kollektors, dargestellt als logarithmische Regressionsgeraden, für ausgewählte sonnige Tage im Jahresverlauf in der ersten Tageshälfte in Abhängigkeit von der Globalstrahlung in Kollektorebene

Abbildung 23: Geförderter Luftvolumenstrom der Kollektorfelder des Solardach-Luft-Kollektors mit Mittelwerten der einzelnen Versuchsgruppen.

Abbildung 24: Einfluss der untersuchten Faktoren des Solardach-LuftKollektors auf den geförderten Luftvolumenstrom .63

Abbildung 25: Luftgeschwindigkeiten in den Anlagenabschnitten des Solardach-Luft-Kollektors

Abbildung 26: Luftgeschwindigkeiten in den unterschiedlichen Anlagenabschnitten bei nur einem geöffneten Kollektorfeld

Abbildung 27: Thermische Nutzenergie des Solardach-Luft-Kollektors im Jahresverlauf bei einer Abkühlung der Lufttemperatur auf $10^{\circ} \mathrm{C}$ 66

Abbildung 28: Bereinigter thermischer Energiegehalt der Umgebungsluft im Jahresverlauf bei einer Abkühlung der Lufttemperatur auf $10^{\circ} \mathrm{C}$ 
Abbildung 29: Nutzungszeitraum der bereinigten thermischen Nutzenergie des Solardach-Luft-Kollektors und der bereinigten thermischen Nutzenergie der Umgebungsluft bei einer Abkühlung der Lufttemperatur auf $10^{\circ} \mathrm{C}$

Abbildung 30: Energieanteile an der bereinigten thermischen Nutzenergie des Solardach-Luft-Kollektors im Jahresverlauf bei einer Abkühlung der Lufttemperatur auf $10^{\circ} \mathrm{C}$ 69

Abbildung 31: Zusätzlich gewonnene thermische Energie durch den Solardach-Luft-Kollektor ohne den Energiegehalt der Umgebungsluft im Jahresverlauf bei einer Abkühlung der Lufttemperatur auf $10^{\circ} \mathrm{C}$

Abbildung 32: Bereinigte theoretisch mögliche Gesamtnutzenergie im Jahresverlauf

Abbildung 33: Thermischer Energiebedarf der Jugendherberge im Jahresverlauf 2008/2009

Abbildung 34: Abgenommene Wärmemenge der Jugendherberge $2008 / 2009$ 


\section{Tabellenverzeichnis}

Tabelle 1: Mittlere Luftvolumenströme der Kollektorfelder .62

Tabelle 2: Vergleich der bereinigten möglichen Gesamtnutzenergie mit der bereinigten Kollektornutzenergie für verschiedene Monate

Tabelle 3: Simulierte Wärmetauschertemperaturen für verschiedene Jahreszeiten

Tabelle 4: Leistungszahlen des Verdichters für verschiedene Jahreszeiten und Temperaturniveaus. 76

Tabelle 5: Arbeitszahlen bei vollständiger Wärmeabnahme für verschiedene Jahreszeiten und Temperaturniveaus.

Tabelle 6: Jahresarbeitszahlen bei vollständiger Wärmeabnahme für verschiedene Temperaturniveaus

Tabelle 7: Warmwasserverbrauch in der Jugendherberge 2008/2009

Tabelle 8: Wärmepumpenlaufzeiten bezogen auf die Wärmeabnahme in der Jugendherberge für verschiedene Jahreszeiten

Tabelle 9: Arbeitszahlen bezogen auf die Wärmeabnahme in der Jugendherberge für verschiedene Jahreszeiten

Tabelle 10: Jahresarbeitszahl bezogen auf die Wärmeabnahme in der Jugendherberge

Tabelle 11: Leistungszahlen des Verdichters bei einem optimierten Wärmepumpenbetrieb für verschiedene Jahreszeiten und Temperaturniveaus

Tabelle 12: Arbeitszahlen bei einem optimierten Wärmepumpenbetrieb für verschiedene Jahreszeiten und Temperaturniveaus

Tabelle 13: Jahresarbeitszahlen bei einem optimierten Wärmepumpenbetrieb für verschiedene Temperaturniveaus 
Tabelle 14: Investitionskosten der solaren Energiegewinnung .88

Tabelle 15: Energiebezugskosten für verschiedene Jahreszeiten und Temperaturniveaus bei einer vollständigen Wärmeabnahme

Tabelle 16: Eingesparte Jahresenergiebezugskosten bei einer vollständigen Wärmeabnahme

Tabelle 17: Amortisationszeitraum der Investitionskosten für die solare Energiegewinnung bei einer vollständigen Wärmeabnahme .....90

Tabelle 18: Energiebezugskosten für verschiedene Jahreszeiten bezogen auf die Wärmeabnahme in der Jugendherberge

Tabelle 19: Eingesparte Jahresenergiebezugskosten bezogen auf die Wärmeabnahme in der Jugendherberge .92

Tabelle 20: Amortisationszeitraum der Investitionskosten für die solare Energiegewinnung bezogen auf die Wärmeabnahme in der Jugendherberge

Tabelle 21: Energiebezugskosten für verschiedene Jahreszeiten und Temperaturniveaus bei einer Optimierung des Wärmepumpenbetriebes bezogen auf eine vollständige Wärmeabnahme

Tabelle 22: Eingesparte Jahresenergiebezugskosten für die Optimierung bei vollständiger Wärmeabnahme .95

Tabelle 23: Amortisationszeitraum der Investitionskosten für die solare Energiegewinnung bei einer Optimierung des Wärmepumpenbetriebes bezogen auf eine vollständige Wärmeabnahme 


\section{Symbol- und Abkürzungsverzeichnis}

\begin{tabular}{|c|c|c|}
\hline A : & Fläche & {$\left[\mathrm{m}^{2}\right]$} \\
\hline Abb.: & Abbildung & \\
\hline$a_{f}:$ & Anlagenfaktor & {$[-]$} \\
\hline $\mathrm{A}_{\mathrm{Q}}:$ & Querschnittsfläche & {$\left[\mathrm{m}^{2}\right]$} \\
\hline $\mathrm{A}_{\mathrm{R}}:$ & Rohrquerschnittsfläche & {$\left[\mathrm{m}^{2}\right]$} \\
\hline $\mathrm{A}_{\mathrm{r}}$ : & rechteckige Querschnittsfläche & {$\left[\mathrm{m}^{2}\right]$} \\
\hline AW: & Ausgangswert & {$\left[\mathrm{m}^{3} / \mathrm{h}\right]$} \\
\hline aWM: & abgegebene Wärmemenge & {$[\mathrm{kWh}]$} \\
\hline AZR: & Amortisationszeitraum & [a] \\
\hline $\mathrm{b}:$ & Breite & {$[\mathrm{m}]$} \\
\hline$\beta:$ & Arbeitszahl & {$[-]$} \\
\hline$\beta_{\mathrm{a}}:$ & Jahresarbeitszahl & {$[-]$} \\
\hline BGGA: & Bemessungsgrundlage Gasabrechnung & {$[\mathrm{kWh}]$} \\
\hline BWE: & Brauchwassererwärmung & \\
\hline BWP: & Bundesverband Wärmepumpe e. V. & \\
\hline $\mathrm{C}_{\mathrm{pD}}:$ & spezifische Wärmekapazität Wasserdampf & {$[\mathrm{kJ} /(\mathrm{kg} \cdot \mathrm{K})]$} \\
\hline $\mathrm{c}_{\mathrm{pL}}:$ & spezifische Wärmekapazität Luft & {$[\mathrm{kJ} /(\mathrm{kg} \cdot \mathrm{K})]$} \\
\hline $\mathrm{c}_{\mathrm{pW}}:$ & spezifische Wärmekapazität Wasser & {$[\mathrm{Wh} /(\mathrm{kg} \cdot \mathrm{K})]$} \\
\hline $\mathrm{d}:$ & Rohrdurchmesser & {$[\mathrm{m}]$} \\
\hline$\Delta \mathrm{h}_{\mathrm{v} 0}:$ & Verdampfungsenthalpie bei $0^{\circ} \mathrm{C}$ & {$[\mathrm{kJ}]$} \\
\hline$\Delta \mathrm{T}:$ & Temperaturdifferenz & {$[\mathrm{K}]$} \\
\hline
\end{tabular}


DIN: Deutsches Institut für Normung e. V.

DN: $\quad$ Nennweite

DNZ: Differenz Nutzungszeitraum

[h]

DVGW: $\quad$ Deutsche Vereinigung des

Gas- und Wasserfaches e. V.

DWD: Diffusionsoffene Dach- und Wandplatte

E: $\quad$ East

eAE: $\quad$ elektrische Antriebsenergie

$[\mathrm{kWh}]$

EAL: $\quad$ Elektroantriebsleistung $\quad[\mathrm{kW}]$

ED: $\quad$ Energiedifferenz $\quad[k W h]$

eEBK: $\quad$ eingesparte Energiebezugskosten [€]

EEWärmeG: $\quad$ Erneuerbare-Energien-Wärmegesetz

EN: $\quad$ Europäische Norm

$\varepsilon$ : $\quad$ Leistungszahl

$\varepsilon_{\mathrm{V}}: \quad$ Leistungszahl Verdichter $\quad[-]$

$\mathrm{E}_{\mathrm{th}}: \quad$ abgegebene thermische Energie [kWh]

$\eta$ Wirkungsgrad $\quad[-]$

$\mathrm{E}_{\mathrm{W}}$ : Wärmebedarf Warmwasserbereitung [Wh]

FTP: $\quad$ File Transfer Protocol

GBK: Gasbrennkessel

$\dot{\mathrm{G}}_{\mathrm{g}}$ : $\quad$ Globalstrahlung in der Kollektorebene $\quad\left[\mathrm{kW} / \mathrm{m}^{2}\right]$

GK: Gaskosten [€]

GMB: Gasmengenbedarf $\left[\mathrm{m}^{3}\right]$

GNE: Gesamtnutzenergie [kWh]

GWA: Gesamtwärmeabnahme [kWh] 


\begin{tabular}{|c|c|c|}
\hline h : & Höhe & [m] \\
\hline HP: & Heizungspufferspeicher & \\
\hline $\mathrm{h}_{1+\mathrm{x}}:$ & Enthalpie des ungesättigten Gemisches & {$[\mathrm{kJ} / \mathrm{kg}]$} \\
\hline $\mathrm{h}_{1+\mathrm{x}_{\text {Aus }}}:$ & Austrittsenthalpie der Luft & {$[\mathrm{kJ} / \mathrm{kg}]$} \\
\hline $\mathrm{h}_{1+\mathrm{x}_{\mathrm{Ein}}}:$ & Eintrittsenthalpie der Luft & {$[\mathrm{kJ} / \mathrm{kg}]$} \\
\hline $\mathrm{I}_{\mathrm{A}}$ : & Ausgangsstrom & {$[\mathrm{A}]$} \\
\hline $\mathrm{I}_{\mathrm{A}_{\left(30^{\circ} \mathrm{C}\right)}}$ : & Ausgangsstrom bei $30^{\circ} \mathrm{C}$ & {$[\mathrm{A}]$} \\
\hline IK: & Investitionskosten & {$[€]$} \\
\hline ISG: & Insektenschutzgitter & \\
\hline KF: & Kollektorfeld & \\
\hline KG-Rohr: & Kanalgrundrohr & \\
\hline KNE: & Kollektornutzenergie & [kWh] \\
\hline kPS: & kältester Pfufferspeicher & {$\left[{ }^{\circ} \mathrm{C}\right]$} \\
\hline l: & Länge & {$[\mathrm{m}]$} \\
\hline LA: & Leistungsaufnahme & \\
\hline LAN: & Local Area Network & \\
\hline LAT: & Luftaustrittstemperatur & {$\left[{ }^{\circ} \mathrm{C}\right]$} \\
\hline LET: & Lufteintrittstemperatur & {$\left[{ }^{\circ} \mathrm{C}\right]$} \\
\hline LVS: & Luftvolumen & {$\left[\mathrm{m}^{3} / \mathrm{h}\right]$} \\
\hline LZ: & Laufzeit & [h] \\
\hline $\mathrm{m}:$ & Masse & {$[\mathrm{kg} / \mathrm{d}]$} \\
\hline M-Bus: & Meter-Bus & \\
\hline min: & Minuten & \\
\hline$\dot{\mathrm{m}}_{\mathrm{L}}:$ & Massenstrom der Luft & {$[\mathrm{kg} / \mathrm{s}]$} \\
\hline
\end{tabular}


MMV:

Mischwasserverbrauch

[l]

MOP: $\quad$ Maximum Operating Pressure

MW:

mWLZ:

$\mathrm{N}$ :

$N+F$ :

NZGNE:

NZKNE:

$\varnothing$ :

PBVE:

$\mathrm{P}_{\mathrm{el}}$ :

$\varphi:$

$\pi:$

$\mathrm{p}_{\mathrm{L}}$ :

$\mathrm{p}_{\mathrm{s}}$ :

$\mathrm{P}_{\text {th }}$ :

$\dot{\mathrm{Q}}_{0}:$

RAL:

rLF:

RS:

SKWP:

SRT:

SSP:

STAW:
Mittelwert

$\left[\mathrm{m}^{3} / \mathrm{h}\right]$

[h]

North

Nut und Feder

Nutzungszeitraum Gesamtnutzenergie

[h]

Nutzungszeitraum Kollektornutzenergie

[h]

Durchmesser

Pufferspeicher Brauchwasservorerwärmung

elektrische Antriebsleistung

[W]

relative Luftfeuchtigkeit

$[-]$

$\mathrm{Pi}$

$[-]$

barometrischer Luftdruck

[Pa]

Sättigungsdampfdruck

[Pa]

abgegebene thermische Leistung

[kW]

Verdampferleistung

[W]

Farbtonnormung des Deutschen Instituts

für Gütesicherung und Kennzeichnung e. V.

relative Luftfeuchtigkeit

[\%]

Rohrsystem

Stromkosten Wärmepumpe

[€]

Solerücklauftemperatur

$\left[{ }^{\circ} \mathrm{C}\right]$

Solespreizung

[K]

Standardabweichung 
SVT:

$\mathrm{T}_{\mathrm{a}}$ :

Tab.:

$\mathrm{T}_{\mathrm{e}}$ :

$t_{L}$ :

ÜN:

UP:

UTC:

VDI:

$\mathrm{v}_{\mathrm{L}}$ :

$\dot{\mathrm{V}}_{\mathrm{L}}$ :

$\mathrm{VL}$ :

WBD:

$\mathrm{W}_{\mathrm{el}}$ :

WL:

WLZ:

WMZ:

$\mathrm{W}_{\text {Nutz }}$ :

WP:

WT:

WWV:

$\mathrm{x}_{\mathrm{L}}$ :

$\mathrm{Z}$ :
Solevorlauftemperatur

Austrittstemperatur

Tabelle

Eintrittstemperatur

Lufttemperatur

Übernachtungen

Umwälzpumpe

Universal Time Coordinated

Verein Deutscher Ingenieure e. V.

Luftgeschwindigkeit

spezifischer Luftvolumenstrom

Verdampferleistung

Wärmebedarfsdeckung

gesamte elektrische Antriebsenergie

Wärmeleistung

Wärmepumpenlaufzeit

Wärmemengenzähler

abgegebene Nutzwärme

Wärmepumpe

Wärmetauscher mit Trichter

Warmwasserverbrauch

Feuchtegehalt der Luft

Messintervall
[kWh]

$\left[{ }^{\circ} \mathrm{C}\right]$

[K]

[K]

$\left[{ }^{\circ} \mathrm{C}\right]$

$[\mathrm{m} / \mathrm{s}]$

$\left[\mathrm{m}^{3} / \mathrm{s}\right]$

[kW]

[kWh]

[kWh]

[kW]

[h]

[l]

$\left[\mathrm{kg}_{\mathrm{H}_{2} \mathrm{O}} / \mathrm{kg}_{\text {Luft }}\right]$

[h] 


\section{$1 \quad$ Einleitung}

Angesichts der steigenden Energiepreise und der zum 1. Januar 2009 eingeführten Nutzungspflicht von erneuerbaren Energien gewinnt in diesem Kontext die Sonnenenergie zunehmend an Bedeutung [6, 9]. Eine bekannte und vielfach angewandte Technologie ist die Nutzung von solarer Strahlungsenergie mit Hilfe von Kollektoren oder photovoltaischen Zellen. Die Umsetzung dieser kostenintensiven Lösungen ist jedoch derzeit nicht ohne finanzielle Förderung wirtschaftlich [57]. Weniger bekannt ist die kostengünstige Verwertung der Sonnenstrahlung durch die Nutzung der unter Dachflächen entstehenden Stauwärme. Bereits in den 80er Jahren konnte durch Grimm (1984) und Lücke (1984) nachgewiesen werden, dass mit handelsüblichen Metallprofilen eingedeckte Dachflächen in Verbindung mit einer Wärmepumpe zur Brauchwassererwärmung geeignet sind [34, 56]. Diese Alternative bietet sich besonders für Gebäude mit großen Dachflächen an. Die Vorteile der Stauwärmenutzung werden vornehmlich in einer erheblichen Kostenreduktion der solarthermischen Energiegewinnung gesehen. Die aktuellen Entwicklungen auf dem Energiemarkt gaben Anlass, das Konzept der Stauwärmenutzung erneut aufzugreifen und einem Praxistest zu unterziehen. Für die Praxiserprobung wurde der Neubau der Jugendherberge des Deutschen Jugendherbergswerkes in Dahme (Ostsee/Deutschland) mit einem Prototyp des Solardach-Luft-Kollektors mit Metalleindeckung ausgestattet. Das Solardach entspricht systematisch einem Solar-Luft-Kollektor ohne Abdeckung (Absorber), der in der Jugendherberge in Verbindung mit einer Wärmepumpe zur Brauchwasservorerwärmung und Heizungsunterstützung eingesetzt wird. Das Gebäude verfügt über große Dachflächen, die eine Wärmenutzung sinnvoll erscheinen lassen.

In der vorliegenden Arbeit geben die ersten Kapitel einen Überblick über den Stand des Wissens. Nach diesen Kapiteln wird das Betriebsverhalten der bestehenden Pilotanlage anhand von Untersuchungen bestimmt. Es werden zunächst Untersuchungen an dem Solardach-Luft-Kollektor durchgeführt und 
im Anschluss das dahinterstehende Nutzungssystem beurteilt. Es wird davon ausgegangen, dass Dachflächen, die mit handelsüblichen Metalldachprofilen eingedeckt sind, sich zur thermischen Energiegewinnung eignen und in Verbindung mit einer Wärmepumpe zur Brauchwassererwärmung und Heizungsunterstützung einsetzbar sind. Weiterhin wird erwartet, dass sich die Stauwärmenutzungsanlage für einen ökonomischen Betrieb in der Jugendherberge Dahme eignet. 


\section{2}

\section{Literaturübersicht}

\subsection{Einteilungsmöglichkeiten und besondere Eigenschaften von Solar-Luft-Kollektoren}

Der Hauptbestandteil eines Solar-Luft-Kollektors ist der Absorber, der die einfallende Solarstrahlung aufnimmt und in Form von Wärme an das Wärmeträgermedium Luft abgibt. Solar-Luft-Kollektoren können anhand der Absorberumströmung sowie in abgedeckte und nicht abgedeckte Kollektoren eingeteilt werden. Die Literatur nimmt weitere Unterteilungen nach Herstellungsart, dem Absorbermaterial und der Absorberbeschichtung vor, auf die jedoch im Folgenden nicht weiter eingegangen wird [28, 40, 44].

Die unterschiedlichen Arten der Absorberumströmungen sind von der jeweiligen Bauart des Solar-Luft-Kollektors abhängig. In Abbildung 1 werden die möglichen Absorberumströmungen am Beispiel eines abgedeckten SolarLuft-Kollektors dargestellt. Der überströmte Absorber liegt auf der Wärmedämmung auf und die Luft strömt zwischen dem Absorber und der transparenten Abdeckung durch den Kollektor. Die bei dieser Bauart entstehenden vorderseitigen Wärmeverluste werden durch einen unterströmten Absorber reduziert. Bei dieser Kollektorbauform strömt die Luft zwischen der Wärmedämmung und dem Absorber durch den Kollektor, ohne mit der transparenten Kollektorabdeckung in Berührung zu kommen. Der umströmte Absorber wird von der Ober- und Unterseite umströmt und erzielt dadurch einen verbesserten Wärmeübergang vom Absorber zum Wärmeträger. Durchströmte Absorber bestehen aus einer porösen Platte, die von vorne nach hinten durchströmt wird. Diese Absorberumströmung reduziert die vorderseitigen Wärmeverluste, wird in der Literatur jedoch als anfällig für Verunreinigungen beschrieben [25, 28, 40, 61]. 


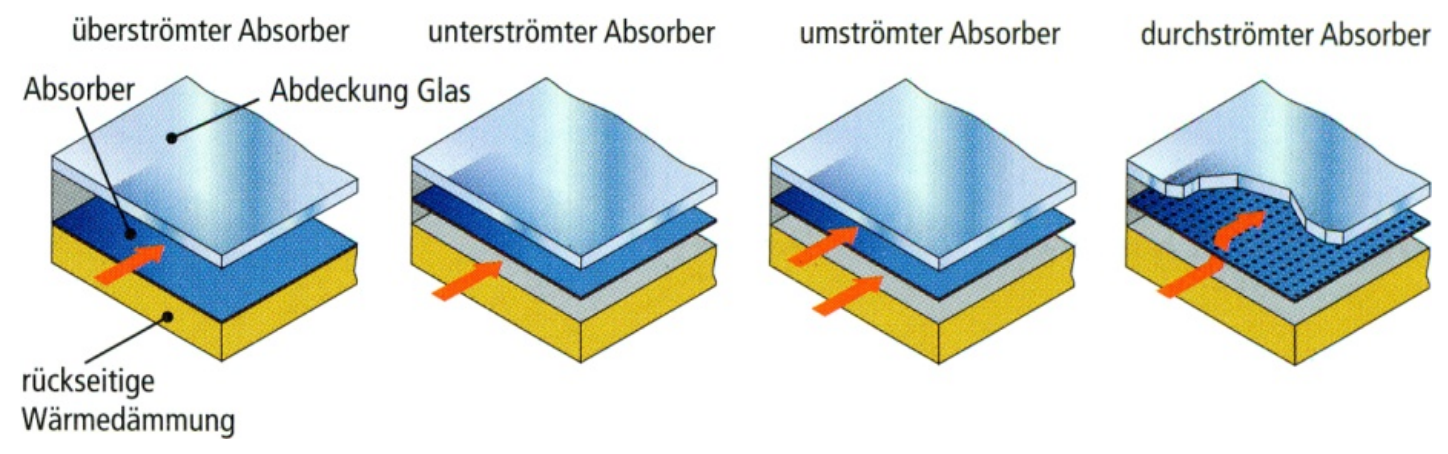

Abbildung 1: Arten der Absorberumströmung, dargestellt am Beispiel eines abgedeckten Solar-Luft-Kollektors (Heidler et al. 2008 [40])

Die Absorberumströmungen, unter- und durchströmt, sind bei nicht abgedeckten Kollektoren möglich. Nicht abgedeckte Kollektoren besitzen keine transparente Abdeckung und werden in der Literatur als Absorber bezeichnet $[28,40]$. Ein Absorber ist ein Flachkollektor, der direkt der Umgebungsluft ausgesetzt ist und in der Regel keine Wärmedämmung besitzt [43]. Dadurch sind die Wärmeverluste gegenüber abgedeckten Kollektoren deutlich höher. Absorber eignen sich aufgrund der fehlenden Abdeckung und der daraus resultierenden guten Anpassungsfähigkeit besonders zur Integration in Dachflächen [40]. Dachflächen mit integrierten Kollektoren (Absorbern) werden als Solardächer bezeichnet $[34,56,57]$. Bei Solardach-Luft-Kollektoren ersetzt ein aus Metall bestehender dunkel eingefärbter Absorber die Dacheindeckung des Solardaches $[34,56]$.

Solardach-Luft-Kollektoren sind aufgrund ihres Wärmeträgermediums Luft nicht so leistungsfähig wie flüssigkeitsdurchströmte Flachkollektoren und arbeiten auf einem niedrigen Temperaturniveau. Dies liegt neben der fehlenden transparenten Abdeckung in den physikalischen Eigenschaften der Luft begründet, die einige Nachteile gegenüber flüssigen Wärmeträgern, wie zum Beispiel Wasser, mit sich bringen. Dies ist zum einen die geringe spezifische Wärmekapazität der Luft. Daraus resultiert, dass für den Wärmetransport hohe Massen- bzw. Volumenströme notwendig sind. Zum anderen ist der Wärmeübergang vom Absorber bei Luftkollektoren durch die geringe Wärmeleitfähigkeit der Luft schlechter als bei ähnlich aufgebauten 
flüssigkeitsdurchströmten Flachkollektoren. Dies hat ebenfalls zur Folge, dass für die Wärmeübertragung auf andere Medien große Übertragungsflächen erforderlich sind. Des Weiteren eignet sich Luft nicht als Speichermedium aufgrund der geringen Wärmekapazität der Luft.

Bedingt durch diese physikalischen Eigenschaften haben Luftkollektoren eine geringere spezifische Leistung als Flachkollektoren und beanspruchen deshalb für die solarthermische Energiegewinnung eine größere Fläche $[28,40,52]$.

Demgegenüber stehen eine Reihe von Argumenten, die für Luftkollektoren sprechen. Aufgrund der geringeren spezifischen Wärmekapazität erwärmt sich Luft wesentlich schneller als Wasser. Dies ermöglicht bereits die Nutzung kurzer Sonnenscheinphasen zur Energiegewinnung. Der Aufbau eines Luftkollektors ist im Vergleich zu flüssigkeitsdurchströmten Kollektoren einfach. Aufgrund der physikalischen Eigenschaften von Luft sind keine sicherheitstechnischen Vorkehrungen bezüglich Frostschutz und Stillstandverhalten notwendig. Durch eine geringere Korrosivität der Luft erhöht sich die Lebensdauer von Luftkollektoren gegenüber flüssigkeitsführenden Kollektoren. Der einfache Aufbau und die Einsparung der eigentlichen Dacheindeckung reduzieren die Kosten von Solardach-Luft-Kollektoren gegenüber Flachkollektoren erheblich [28, 34, 40].

\subsection{Umwandlung des Strahlungsangebotes in einem Solardach-Luft-Kollektor}

Die auf die Erde treffende Solarstrahlung setzt sich aus der direkten Strahlung (gerichtete Sonnenstrahlung) und der diffusen Strahlung (Himmelsstrahlung) zusammen [44].

Der Aufbau des Solardach-Luft-Kollektors besteht aus dem dunkel eingefärbten, gut wärmeleitenden Absorber (Metallblech), einem luftdurchströmten Zwischenraum und dem Kollektorboden. Der Absorber hat die Aufgabe, die einfallende Solarstrahlung zu absorbieren und in thermische Energie umzu- 
wandeln. Durch die absorbierte Solarstrahlung erwärmt sich der Absorber und gibt Wärme an die durch den Kollektor strömende Luft ab [40].

Die Wärmeübertragung erfolgt im Allgemeinen durch Konvektion, Wärmeleitung und Wärmestrahlung und wird durch Temperaturdifferenzen ausgelöst. Dabei ist die Wärme stets bestrebt, von einem Medium mit höherer Temperatur auf ein Medium mit niedriger Temperatur überzugehen [43]. Konvektion ist der Wärmetransport zwischen einem strömenden Medium und einem festen Körper. Es wird zwischen der natürlichen und erzwungenen Konvektion unterschieden. Die natürliche Konvektion entsteht durch die Auftriebskraft, die durch Dichteunterschiede hervorgerufen wird. Bei der erzwungenen Konvektion erfolgt die Strömung durch Druckdifferenzen, die zum Beispiel durch einen Ventilator erzeugt werden $[40,43,44]$. Der Wärmefluss in einem festen Körper oder ruhenden Medium wird als Wärmeleitung bezeichnet. Die Wärmeleitfähigkeit eines Stoffes ist neben der Temperaturdifferenz von der molekularen Struktur des Stoffes abhängig [40]. Wärmestrahlung ist eine elektromagnetische Strahlung, die nicht an eine Materie gebunden ist. Sie wird von jedem Körper emittiert sowie absorbiert. Die Strahlungseigenschaft eines Köpers ist abhängig von der Stoffart, Temperatur und der Beschaffenheit der Oberfläche [44].

Für einen guten konvektiven Wärmeübergang in Luftkollektoren ist eine möglichst turbulente Luftströmung erforderlich. Die turbulente Strömung ist unter anderem von der Strömungsgeschwindigkeit, Kanalgröße (hydraulischer Durchmesser) und der kinematischen Viskosität der Luft abhängig [43]. Die Berechnung der Reynoldszahl ermöglicht die Beurteilung des Strömungsverhaltens bezogen auf das Vorliegen einer laminaren oder turbulenten Strömung [49]. Eine Verbesserung der Turbulenz kann durch das Anrauen der Absorberunterfläche und Querschnittsverengungen im Strömungskanal erzielt werden [34, 56, 61]. Weiterhin ist es möglich, durch Lamellen oder feine Profilierungen die Oberfläche des Absorbers für einen besseren Wärmeübergang zu vergrößern [34]. 
Die Abbildung 2 zeigt, dass die Umwandlung der Solarstrahlung in thermische Energie mit Verlusten behaftet ist. Optische Verluste entstehen durch Reflexionsverluste der einfallenden Solarstrahlung am Absorber. Die Reflexionsverluste und deren Abhängigkeit vom Einstrahlungswinkel sind jedoch bei nicht abgedeckten Kollektoren relativ gering [40]. Der größte Anteil der Verluste wird durch thermische Verluste hervorgerufen. Der erwärmte Absorber gibt durch Strahlungsaustausch mit der Atmosphäre, Konvektion und durch Wärmeleitung an den Kollektorboden einen nicht mehr nutzbaren Wärmestrom ab [34]. Die Abstrahlungsverluste an die Atmosphäre werden durch die Absorbertemperatur und die Himmelstemperatur beeinflusst [25]. Konvektive Wärmeverluste durch die Außenluft werden durch den Einfluss von Wind verstärkt [25, 40]. Der erwärmte Kollektorboden verursacht Wärmeverluste durch Wärmeübertragungen an den Firstraum. Weiterhin entstehen an den Kollektorseiten Wärmeverluste, die jedoch im Vergleich zur Kollektorbreite gering sind [34].

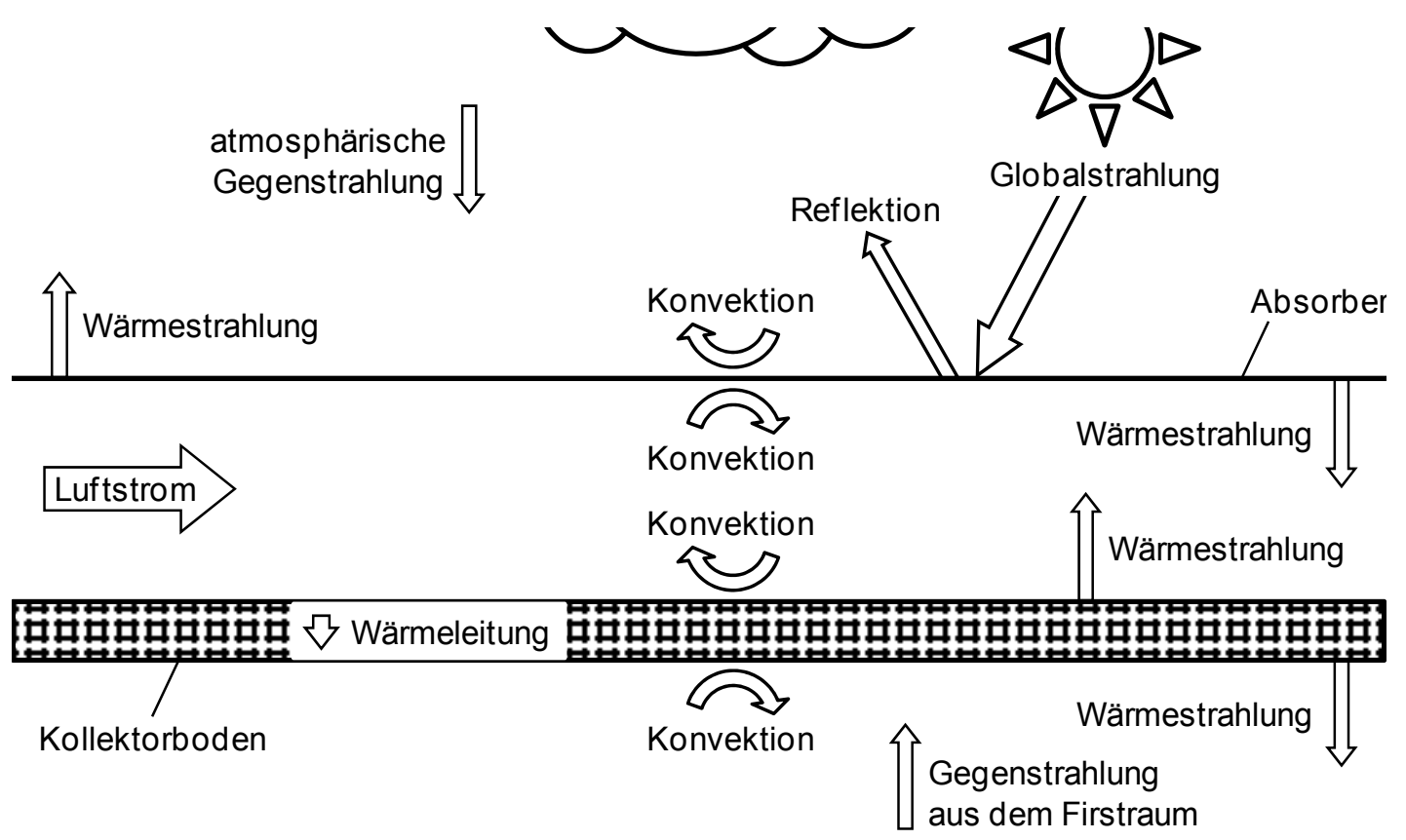

Abbildung 2: Schematische Darstellung der Wärmeübertragung an einem SolardachLuft-Kollektor, verändert nach (Grimm 1984 [35]) 
Das Verhältnis der in Wärme umgewandelten Energie zur eingestrahlten Energie wird durch den Absorptionsgrad ausgedrückt. An den Absorptionsgrad ist nach dem Kirchhoffschen Gesetz bei gleicher Wellenlänge stets ein identischer Emissionsgrad gebunden. Der Emissionsgrad beschreibt das Verhältnis zwischen der absorbierten Energie und der an die Umgebung abgestrahlten Energie. Die Abstrahlleistung des Absorbers steigt nach dem Stefan-Boltzmannschen Gesetz proportional zur vierten Potenz der absoluten Temperatur [44]. Bei höheren Absorbertemperaturen steigen somit die thermischen Verluste aufgrund der zunehmenden Wärmeabstrahlung.

Durch die Verwendung von selektiv beschichteten Oberflächen ist es möglich, diese Verluste zu reduzieren. Die Selektivbeschichtung besitzt ein hohes Absorptionsvermögen und verringert die Abgabe der langwelligen Infrarotstrahlung durch ihre veränderte Schichtstruktur [40,52]. Verluste, die durch Wärmeleitung des Kollektorbodens hervorgerufen werden, können durch eine Dämmung verringert werden [56].

\subsection{Thermische Energiegewinnung mit Solardach-Luft-Kollektoren}

Die thermische Nutzung von Solarenergie mittels verschiedener Kollekorsysteme ist wissenschaftlich weitestgehend untersucht. Im Gegensatz dazu liegen wissenschaftliche Untersuchungen über den Einsatz von Solardach-Luft-Kollektoren nur in einem begrenzten Umfang vor. Einen Überblick über die Möglichkeiten der thermischen Energiegewinnung mit Solar-Luft-Kollektoren bieten Filleux und Gütermann (2005) sowie Hastings und Mørck (2000) [28, 39]. In Europa sind jedoch Solar-Luft-Kollektoren relativ wenig verbreitet. Dies liegt darin begründet, dass der größte Anwendungsbereich der solarthermischen Energiegewinnung die Brauchwassererwärmung ist und sich Solar-Luft-Kollektoren nur bedingt für dieses Anwendungsgebiet eignen [52]. Bei den auf dem Markt angebotenen SolarLuft-Kollektoren handelt es sich nahezu ausschließlich um abgedeckte Kollektorbauformen. Eine Ausnahme stellt ein perforierter, durchströmter 
Absorber dar, der unter dem Namen „SolarWall“ auf dem Markt vertrieben wird. Neben einer Integration in die Gebäudefassade wird die "SolarWall“ ebenfalls als Solardach-Luft-Kollektor angeboten [13, 27, 28, 39].

In der Literatur gibt es einige Studien zu durchströmten Absorbern. Gunnewiek et al. (1996) entwickelte mit Hilfe einer kommerziellen numerischen Strömungssimulation ein analytisches Werkzeug, das die Strömungsverteilung eines nicht abgedeckten Absorbers vorhersagen kann. Diese Untersuchungen basieren auf einem, an einer senkrechten Wand angebrachten, Absorberblech mit gleichmäßig verteilten Löchern. Durch diese Löcher wurde mit Hilfe eines Ventilators Außenluft angesaugt. Die Studie hat gezeigt, dass die Wärmeübertragung an der Rückseite des Absorbers eine entscheidende Rolle spielt und eine turbulente Strömung einen höheren Kollektorwirkungsgrad gegenüber einer linearen Strömung erzielt. In dieser Studie konnte weiterhin die Auswirkung einer nicht einheitlichen Luftströmung hinter dem Absorber dargestellt werden. Die Art des Strömungsprofils ist von der „Polarität“ des Profils abhängig. Je nach Profilart dominiert der natürliche Auftrieb oder die durch den Ventilator hervorgerufene Luftbewegung. Ist die Strömungsgeschwindigkeit im unteren Absorberteil höher als im oberen Teil, ist bei einer turbulenten Strömung eine Wirkungsgraderhöhung zu erwarten. Tritt der umgekehrte Fall ein, ist ein niedriger Kollektorwirkungsgrad wahrscheinlich. Bei der höheren Strömungsgeschwindigkeit im unteren Teil dominiert der natürliche Auftrieb. Um diesen Effekt positiv zu nutzen, sollte der Luftkanal relativ breit, der hydraulische Widerstand des Absorbers relativ gering und die durchschnittliche Saugleistung der Ventilatoren niedrig sein. In der Praxis sollten jedoch Strömungsgeschwindigkeiten unter $0,0125 \mathrm{~m} / \mathrm{s}$ vermieden werden, damit die erwärmte Luft nicht aus dem Kollektor entweichen kann. Bei dieser Studie handelt es sich um ein Modell, welches in experimentellen Untersuchungen noch bestätigt werden muss. In dieser Studie wurde Windstille vorausgesetzt [36].

In einer weiterführenden Studie wurde anhand des zuvor beschriebenen Modells der Einfluss des Windes und der Gebäudeorientierung bei einer 
Windgeschwindigkeit von $5 \mathrm{~m} / \mathrm{s}$ in Gebäudehöhe untersucht. Die Studie hat gezeigt, dass der Einfluss des Windes auf den Rückstrom der erwärmten Luft durch die Anhebung der Strömungsgeschwindigkeit verhindert werden kann. Je nach Gebäude wurde die Strömungsgeschwindigkeit von etwa 0,0125 m/s auf bis zu 0,03 m/s angehoben. Zudem werden mögliche Strategien diskutiert, die eine Verringerung der benötigten Saugleistung durch die Ventilatoren hervorrufen könnten. Als eine Strategie wird angeführt, den Bereich knapp unterhalb der Dachlinie zu verlassen, da dort der größte Einfluss bei zunehmender Windgeschwindigkeit lokalisiert wurde. Eine weitere Möglichkeit wäre, die Windgeschwindigkeit zu erfassen und die Strömungsgeschwindigkeit den jeweiligen Bedingungen anzupassen. Für die abschließenden Beurteilungen dieser Strategien sind weitere Untersuchungen notwendig [37].

Deans und Weerakoon (2008) untersuchten ebenfalls einen perforierten, durchströmten Absorber. In ihrer experimentellen Studie kommen sie zu dem Ergebnis, dass die Vermischung der warmen Luft an der Rückseite des Absorbers die Wärmeübertragung steuert. Als die wichtigsten Variablen in Bezug auf den Kollektorwirkungsgrad nennen Deans und Weerakoon (2008) die Strömungsgeschwindigkeit und die in Kollektorebene vorhandene Globalstrahlung. Dabei ist die Strömungsgeschwindigkeit die einzige steuerbare Variable, um einen höheren Energietransfer oder eine höhere Temperaturdifferenz zu erzielen [22]. Daraus resultiert: Je höher die Luftgeschwindigkeit im Kollektor ist, desto höher ist der Wirkungsgrad. Höhere Temperaturdifferenzen können hingegen durch möglichst geringe Luftgeschwindigkeiten erzielt werden.

Für durchströmte Absorber sind weitere Studien bekannt, diese haben jedoch für diese Arbeit keine größere Bedeutung [1, 51, 50, 73].

Belusko et al. (2004) beschäftigt sich in seinen Untersuchungen mit der Fragestellung, ob mit kostengünstig hergestellten Solardach-Luft-Kollektoren eine ökonomisch sinnvolle Energieeinsparung möglich ist. Dazu wurde in einem mathematischen Modell die Effizienz von einem nicht verglasten und 
einem verglasten Solardach-Luft-Kollektorsystem einer Wärmepumpe gegenübergestellt. Ein Ergebnis war, dass ohne Verglasung keine sinnvolle Energieeinsparung generiert werden konnte. Bei der verglasten Variante hingegen konnte die Leistung gegenüber einer Wärmepumpe bis zu $22 \%$ verbessert werden. Stellt man jedoch die Kapitalkosten der Leistung gegenüber, zeigt sich, dass das verglaste Solardach-Luft-Kollektorsystem eine niedrigere Leistung besitzt. Die Studie zeigt auf, dass weiterer Forschungsbedarf notwendig ist. In einer experimentellen Studie sind die Ergebnisse zu validieren und Möglichkeiten der Kostenreduktion sowie Optimierung der thermischen Leistung zu prüfen [2].

Keller und Kyburz (1987) untersuchten fünf verschiedene Solardach-LuftKollektoren an einem Freiluftprüfstand. Dabei fanden sie u. a. heraus, dass der Wirkungsgrad bei Erhöhung des Luftvolumenstroms steigt. Dagegen wurde ein abnehmender Wirkungsgrad bei zunehmenden Kollektorlängen und Kanalhöhen festgestellt. Weiterhin fanden sie heraus, dass der zunehmende hydraulische Druck bei geringen Kanalhöhen und einem hohen Luftvolumenstrom die Wirkungsgradzunahme begrenzt. Kollektoren mit transparenter Abdeckung wiesen bei ihren Untersuchungen einen höheren Wirkungsgrad gegenüber nicht abgedeckten Kollektoren auf. Umwelteinflüsse wurden bei diesen Untersuchungen durch parallele Messungen an einem Referenzkollektor eliminiert [42].

Die experimentellen und analytischen Studien zeigen, dass im Bereich der Solardach-Luft-Kollektoren weiterer Forschungsbedarf besteht. Bei den meisten Studien wurde das komplexe System durch vorher festgelegte Parameter stark vereinfacht. Umfangreiche wissenschaftliche Studien, die die Betriebscharakteristika der verschiedenen Solar-Luft-Kollektoren in der praktischen Anwendung bei unterschiedlichen Witterungsbedingungen gegenüberstellen, fehlen. Einheitliche Prüfverfahren unter standardisierten Bedingungen sind nicht vorhanden. Ein Vergleich ist deshalb nur schwer möglich, da angegebene Wirkungsgrade und Temperaturdifferenzen demzufolge einer großen Schwankungsbreite unterliegen. 
Unverglaste Solar-Luft-Kollektoren erreichen je nach Globalstrahlungsangebot und Luftvolumenstrom Wirkungsgrade von über $60 \%$ und eine Temperaturdifferenz von über 30 Kelvin $[35,39,56]$. Verglaste Solar-LuftKollektoren sind aufgrund geringerer Wärmeverluste effektiver und erreichen hingegen Wirkungsgrade von über $75 \%$ und Temperaturerhöhungen von bis zu 80 Kelvin [38, 39]. Diese Kollektorbauformen sind jedoch im Gegensatz zu unverglasten Solar-Luft-Kollektoren erheblich kostenintensiver [57].

Das Anwendungsgebiet von Solardach-Luft-Kollektoren erstreckt sich vornehmlich auf die Konditionierung von Luftheizungssystemen, die zur Deckung des Heizwärmebedarfes von Gebäuden genutzt werden [26, 52]. Ein weiteres Anwendungsgebiet ist die Trocknung von landwirtschaftlichen Produkten [52, 76]. Neben der direkten Nutzung der erwärmten Luft ist die indirekte Nutzung mittels eines Luft-/Wasserwärmetauschers zur Brauchwassererwärmung möglich [27, 39]. Für dieses Anwendungsgebiet müssen jedoch einige Anforderungen erfüllt werden.

Laut der Trinkwasserverordnung $\S 4$ Absatz 1 gilt für öffentliche Einrichtungen: „Wasser für den menschlichen Gebrauch muss frei von Krankheitserregern, genusstauglich und rein sein. Dieses Erfordernis gilt als erfült, wenn bei der Wassergewinnung, der Wasseraufbereitung und der Verteilung die allgemein anerkannten Regeln der Technik eingehalten werden und das Wasser für den menschlichen Gebrauch den Anforderungen der $\S \S 5$ bis 7 entspricht" [5]. Die allgemein anerkannten Regeln der Technik besagen, dass für den Legionellenschutz in Brauchwasserspeichern eine Mindesttemperatur von $60^{\circ} \mathrm{C}$ eingehalten werden muss. Weiterhin ist gefordert, dass die Brauchwassertemperatur in keiner Stelle des Systems unter $55^{\circ} \mathrm{C}$ fallen darf. Dies bedeutet, dass die entnommene Warmwassermenge erst an der Mischbatterie der Entnahmestelle abgemischt werden darf [19, 20].

Bei Solar-Luft-Kollektoren reicht demzufolge das durchschnittlich zu realisierende Temperaturniveau nicht aus, um den Anforderungen der Trinkwasserverordnung zu genügen. Mit einer Wärmepumpe ist es jedoch möglich, die bereitgestellte Energie des Solardach-Luft-Kollektors, unter Auf- 
wendung von Arbeit, auf ein höheres nutzbares Temperaturniveau anzuheben $[45,56]$.

Die elektrisch angetriebene Kompressionswärmepumpe ist die zurzeit am weitesten verbreitete Art der Wärmepumpe, die für Heizzwecke in Deutschland genutzt wird [10,62]. Die Kompressionswärmepumpe arbeitet auf der Grundlage eines thermodynamischen Kreisprozesses, der sich im Idealfall weitestgehend dem Carnotschen Kreisprozess annähert. Als Wärmeträger sind Sole, Luft und Wasser möglich [48]. Bei Wärmepumpen mit Direktverdampfung entfällt der Wärmeträger auf der kalten Seite der Wärmepumpe und das Arbeitsmittel steht im direkten Kontakt mit der Wärmequelle [62].

Das Verhältnis zwischen der abgegebenen Nutzwärmeleistung und der Antriebsleistung des Verdichters der Wärmepumpe wird als Leistungszahl bezeichnet [48]. Die Leistungszahl wird vornehmlich durch die Temperaturdifferenz zwischen der Wärmequelle und der Vorlauftemperatur der Wärmenutzungsanlage beeinflusst [62]. Je geringer die zu überwindende Temperaturdifferenz ist, desto höher ist die Leistungszahl der Wärmepumpe.

Neben der aus der Literatur bekannten Verbesserung der Effektivität des Wärmepumpenprozesses durch mehrstufige Wärmepumpen bieten die verschiedenen Hersteller von elektrisch betriebenen Kompressionswärmepumpen unterschiedliche Modifikationen und Weiterentwicklungen auf dem Wärmepumpenmarkt an [45]. Dazu zählt unter anderem die Heißgasabschöpfung, auch Topladetechnik oder Hot Gas Water Heater Technologie genannt [21,41]. Bei dieser Technik verfügt die Wärmepumpe über drei separate Heizkreisläufe, die durch eine schrittweise Abschöpfung des heißen Gases unterschiedlich hohe Temperaturen realisieren können. Vorteil dieser Technik ist, dass trotz höherer Temperaturen die Leistungszahl der Wärmepumpe nicht negativ beeinflusst wird [55]. Weitere Modifikationen und Weiterentwicklungen werden auf dem Wärmepumpenmarkt angeboten, haben für diese Arbeit jedoch keine größere Bedeutung [59, 70]. 


\section{$3 \quad$ Material und Methoden}

\subsection{Standortbeschreibung}

Die Pilotanlage wurde in dem sonnenreichen Ferienort Dahme (N54 ${ }^{\circ} 12^{\prime}$ 5.911" E11 ${ }^{\circ} 5^{\prime}$ 29.36") an der schleswig-holsteinischen Ostseeküste errichtet. Der Standort gehört dem Landkreis Ostholstein an und liegt einen Meter über dem Meeresspiegel. Die im Folgenden beschriebenen klimatischen Bedingungen des Standortes beziehen sich auf dreijährige Jahresmittelwerte. Der Standort hat eine mittlere Lufttemperatur von 9,8 ${ }^{\circ} \mathrm{C}$. Die Sonnenscheindauer pro Jahr beträgt $1884 \mathrm{~h}$ mit einer mittleren täglichen Globalstrahlung von $120 \mathrm{~W} / \mathrm{m}^{2}$. Der Mittelwert für die Windgeschwindigkeit beläuft sich auf $5,8 \mathrm{~m} / \mathrm{s}$ und die mittlere relative Luftfeuchte beträgt $88 \%$. Die Niederschlagssumme pro Jahr ist $631 \mathrm{l} / \mathrm{m}^{2}$ [23].

\subsection{Aufbau der Pilotanlage}

Für die Praxiserprobung der Pilotanlage wurde der über große Dachflächen verfügende Neubau einer Jugendherberge gewählt. Es handelt sich um ein zweiflügeliges Gebäude (Abb. 3), das mit einer anthrazitgrauen Dacheindeckung aus polyesterbeschichtetem Aluminium versehen ist (50/429 1,0 mm, RAL 7016, Kunstoff-Clip E10, Kalzip GmbH, Koblenz, Deutschland). Die südlich des Turms gelegenen Dachflächen wurden zur Stauwärmenutzung vorbereitet. Zur Praxiserprobung der Stauwärmenutzung wird jedoch lediglich der nach Südosten angestellte Teil des Daches mit einer Fläche von rund $150 \mathrm{~m}^{2}$ genutzt. 


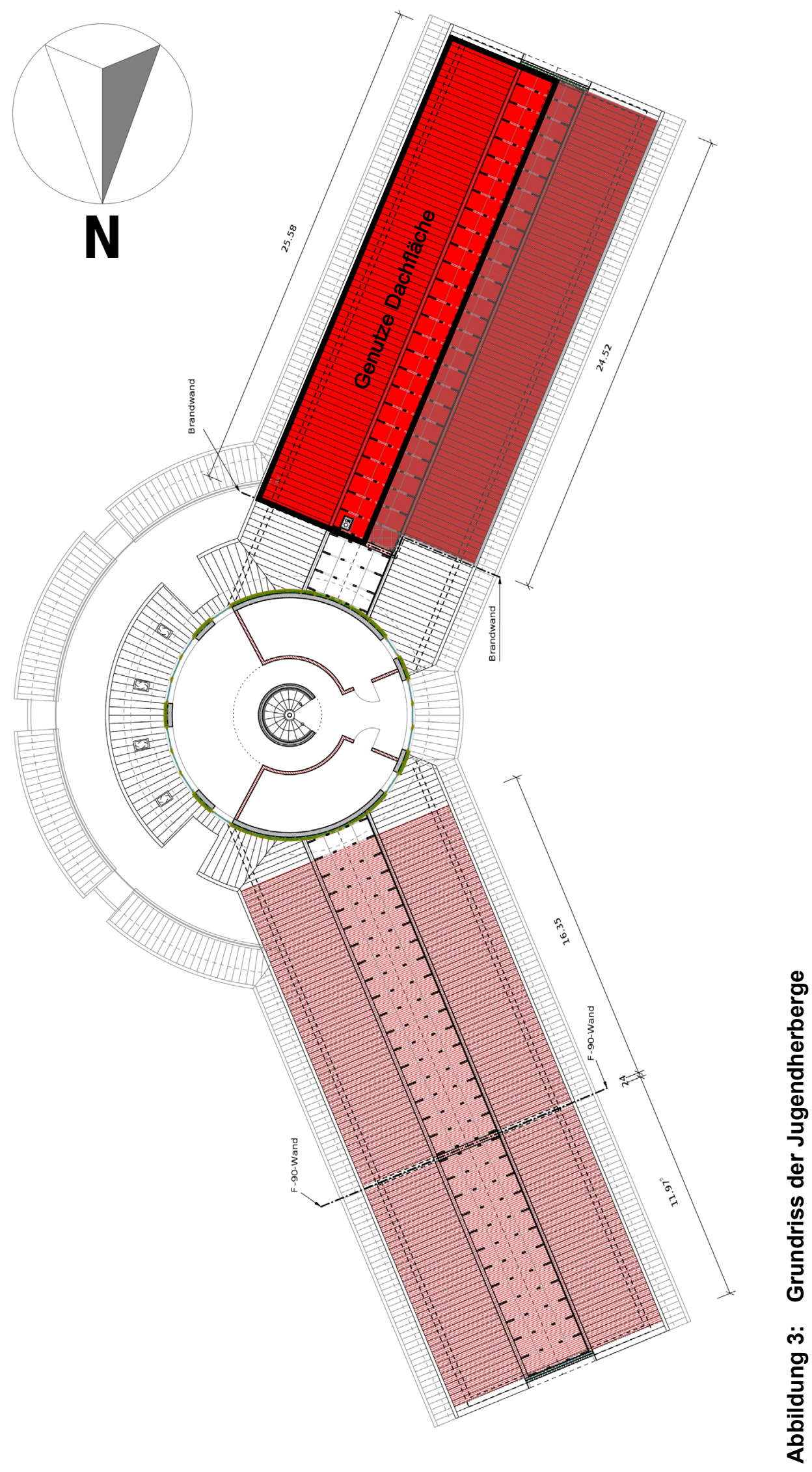


Diese Dachfläche besitzt eine Neigung von $25^{\circ}$ und wurde in fünf voneinander seitlich weitgehend luftdicht abgegrenzte Felder $(5 \times 6 \mathrm{~m})$ aufgeteilt. Jedes dieser Felder (Abb.4) besitzt auf der Unterseite des Daches eine diffusionsoffene Holzwerkstoffplatte (16 mm Agepan DWD N+F, Glunz AG, Meppen, Deutschland), auf deren Oberfläche eine Unterspannbahn (Delta Vent S Plus, Dörken GmbH \& Co. KG, Herdecke, Deutschland) aufgeklebt wurde. Auf dieser Holzwerkstoffplatte wurden in Längsrichtung zur seitlichen Abdichtung und Erzwingung eines geeigneten Luftstromes Konterlatten $(24 \times 60 \mathrm{~mm})$ befestigt. Im Anschluss wurde die eigentliche Dachlattung $(40 \times 80 \mathrm{~mm})$ montiert und das Dach mit Aluminium-Profiltafeln eingedeckt. Der so entstandene ca. 80 mm hohe Strömungskanal wurde in Richtung First verschlossen und an der Traufe mit einem Insektenschutzgitter versehen. Das Insektenschutzgitter besitzt einen Lochanteil von $30 \%$ und soll neben der Schädlingsabwehr eine gleichmäßige Luftdurchströmung im Kollektor gewährleisten.

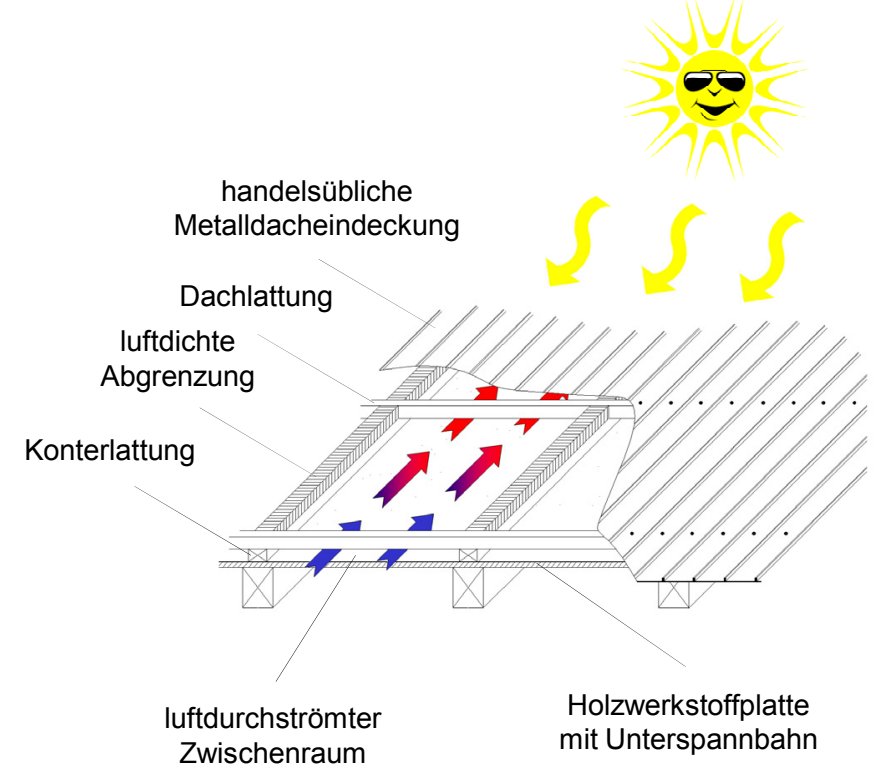

Abbildung 4: Schematischer Aufbau eines Kollektorfeldes des Solardach-LuftKollektors mit Metalleindeckung 
Für die Luftabsaugung wurde jeweils in der Mitte des oberen Drittels der fünf Dachfelder ein Rechteck $(490 \times 100 \mathrm{~mm})$ aus der Holzwerkstoffplatte geschnitten und auf diesen Durchlass anschließend je ein Luftsammeltrichter aufgeschraubt (Abb. 5). Diese Trichter münden jeweils in ein aus flexiblen Schläuchen bestehendes Rohrsystem (DN 200 Clip-Spark, Masterflex AG, Gelsenkirchen, Deutschland), das die erwärmte Luft in das in der Dachspitze verlegte Sammelrohr leitet (DN 200 KG-Rohr, Rehau AG + Co, Rehau, Deutschland). Zwischen die Luftsammeltrichter und das Rohrsystem wurden Lüftungsklappen für die Möglichkeit der Luftstromregulation in den einzelnen Kollektorfeldern integriert.

Das Sammelrohr transportiert die erwärmte Luft der fünf Dachfelder zu einem Wärmetauscher (KS 3228 2,5/700-22RL-5RR-6K-CUAL/111, Wätas Wärmetauscher Sachsen $\mathrm{GmbH}$, Olbernhau, Deutschland), der für einen Luftvolumenstrom von $2.800 \mathrm{~m}^{3} / \mathrm{h}$ mit einer Solespreizung von drei Kelvin ausgelegt ist und zwischen den Dachsparren montiert wurde. Die Luft verlässt den Wärmetauscher über zwei nebeneinander angebrachte Trichter, die jeweils in ein Rohrsystem übergehen (DN 200 KG-Rohr, Rehau AG + Co, Rehau, Deutschland). In Richtung Giebel münden die Rohre in je einen drehzahlregulierbaren Ventilator (CE 670/E 25, Fischbach Luft- und Ventilatorentechnik $\mathrm{GmbH}$, Neunkirchen, Deutschland), der die erwärmte Luft ansaugt und anschließend die abgekühlte Luft über ein Rohrleitungssystem (DN 200 KG-Rohr, Rehau AG + Co, Rehau, Deutschland) dem in der Giebelseite des Dachbodens eingearbeiteten Luftauslass mit Wetterschutzgitter (ø $600 \mathrm{~mm}$ ) zuführt. Die verwendeten Ventilatoren fördern nach Herstellerangaben jeweils einen Luftvolumenstrom von $2.100 \mathrm{~m}^{3} / \mathrm{h}$ bei einer zu überwindenden Gesamtdruckdifferenz von $200 \mathrm{~Pa}$ und bei einer Druckdifferenz von $350 \mathrm{~Pa} 1.545 \mathrm{~m}^{3} / \mathrm{h}$ [29]. In die Rohre vor dem Wärmetauscher wurden zwei Rückschlagklappen (RSK 200, Helios Ventilatoren GmbH + Co KG, Villingen-Schwenningen, Deutschland) eingebaut. Diese Rückschlagklappen sollen verhindern, dass bei nur einem eingeschalteten Ventilator die Luft aus dem gegenüberliegenden Rohr angesaugt wird. 


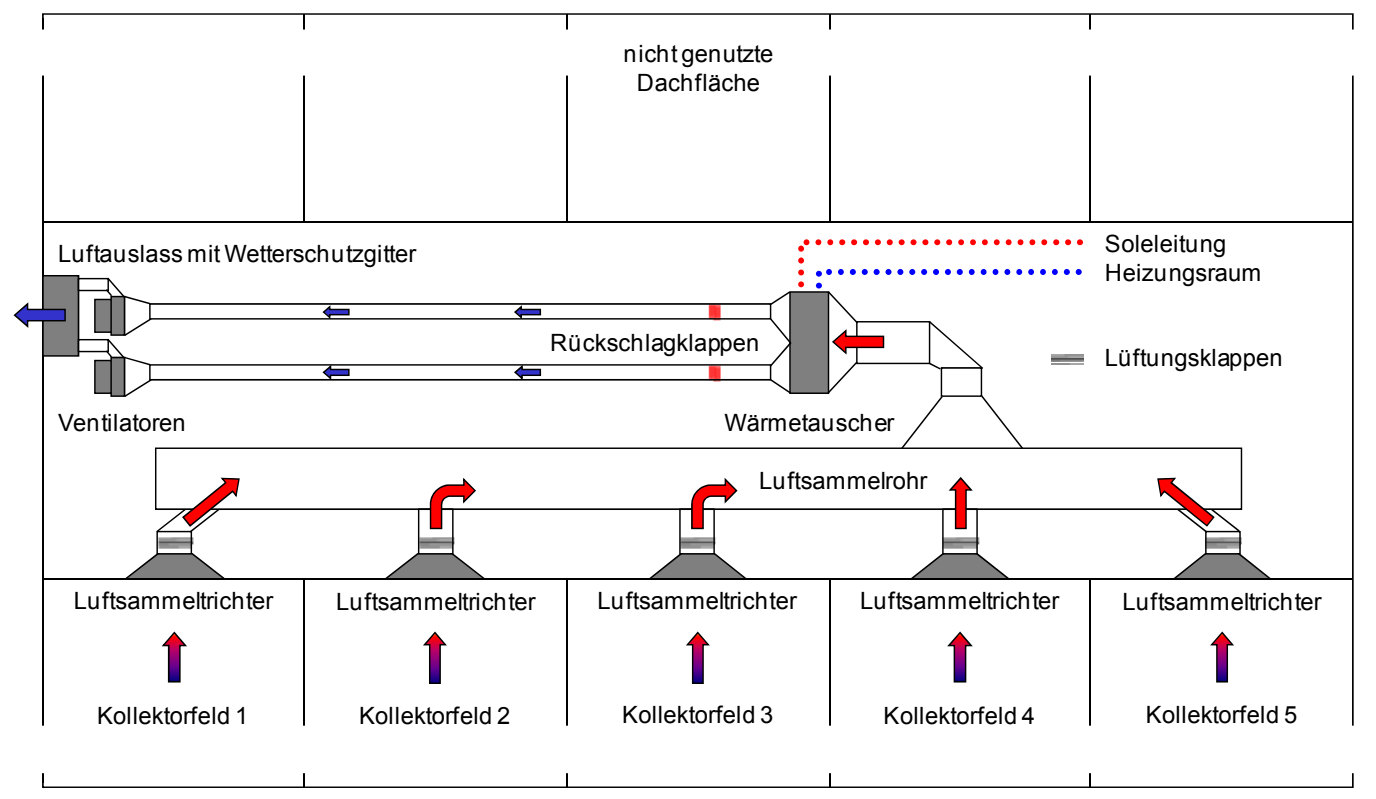

Abbildung 5: Schematische Darstellung der Luftabsaugung

Das gesamte Luftleitsystem wurde zur Vermeidung von Kondensatbildung gedämmt. Zum Abführen des Kondensats aus dem Inneren des Wärmetauschers wurde an der tiefsten Stelle des Wärmtauschers ein Spiralschlauch angebracht. Das Kondensat wird nach dem Schwerkraftprinzip in eine Blechwanne geleitet. Um zu vermeiden, dass das System Fremdluft zieht, muss das Kondensat eine Wassersäule in einem Zylinder überwinden, bevor es in die Blechwanne gelangt. Durch einen Spiralschlauch wird das Kondenswasser über einen integrierten Geruchsverschluss aus der Blechwanne in die Strangentlüftung des Abwassersystems geführt.

Die Ventilatoren wurden zur Schwingungsdämpfung vom Rohrleitungssystem entkoppelt und auf Gummipuffern gelagert. Zusätzlich wurden die Dachsparren durch das Anbringen von Querverstrebungen versteift, um Schwingungsübertragungen in das Gebäude zu vermeiden.

Zur Schallreduktion wurden Geräuschdämmmatten unter den Ventilatoren ausgelegt und die Ventilatorengehäuse von außen gedämmt. Der Wärmetauscher im Dachboden wurde über einen Solekreislauf (Antifrogen $\mathrm{N}$, Clariant Produkte $\mathrm{GmbH}$, Sulzbach, Deutschland) mit einer zweistufigen sauggasgekühlten $13 \mathrm{~kW}$ Sole/Wasser Wärmepumpe mit Heißgas- 
abschöpfung (WPZ 13/2 G S, R 407 C, Karl Santore GmbH, Neuendorf, Deutschland) im Heizungsraum des Kellers verbunden, um die Energie dort auf einem höheren Temperaturniveau an das Heizungssystem abzugeben. Zur Vermeidung einer möglichen Schwingungsübertragung in das Gebäude wurde die Wärmepumpentechnik von der Soleleitung entkoppelt. Die Abbildung 6 zeigt einen vereinfachten Gesamtaufbau der Pilotanlage, der im Weiteren systematisch erläutert wird.

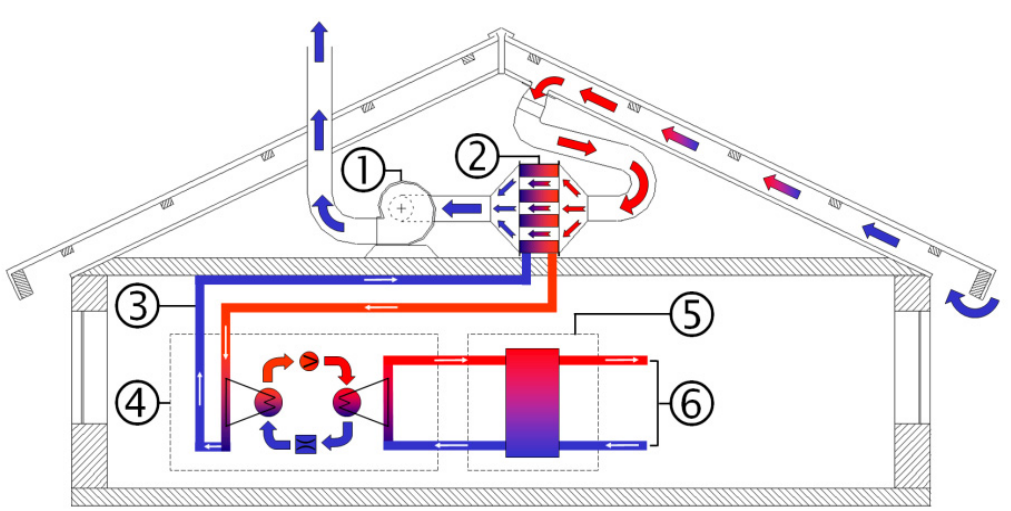

(1) Ventilator

(2) Wärmetauscher

(3) Solekreislauf

(4) Wärmepumpe

(5) Pufferspeicher

(6) Heizungssystem

Abbildung 6: Vereinfacht dargestellter Gesamtaufbau der Pilotanlage

Im Gegensatz zu der vereinfachten Darstellung in Abbildung 6 verfügt die eingesetzte Wärmepumpe über drei separate Heizkreisläufe, die durch eine schrittweise Abschöpfung des heißen Gases unterschiedlich hohe Temperaturen realisieren können. Der erste Heizkreislauf mit dem höchsten Temperaturniveau wurde über einen Pufferspeicher mit 1.000 I (PSM 1000, Austria Email AG, Knittelfeld, Österreich) Fassungsvermögen in das konventionelle Heizungssystem der Jugendherberge integriert (Abb. 7). Die Beladung dieses Heizungspufferspeichers ist jedoch nur möglich, wenn auch das niedrigste Temperaturniveau des letzten Heizkreislaufes der Wärmepumpe abgenommen wird [65]. Um diese Abnahme zu gewährleisten, wurde eine Wärmesenke benötigt. Zu diesem Zweck sind zwei Pufferspeicher (KSW 1051, Huch GmbH Behälterbau, Werder, Deutschland) mit einem Fassungsvermögen von insgesamt 2.100 I zur Brauchwasservorerwärmung installiert worden. Diese beiden Pufferspeicher wurden brauchwasserseitig in 
Serie geschaltet und mit dem zweiten und dritten Heizungskreislauf der Wärmepumpe verbunden. Hydraulisch sind diese Pufferspeicher zur Brauchwassererwärmung so eingebunden, dass bei einer Brauchwasserabnahme das ankommende Frischwasser vom Versorger $\left(10^{\circ} \mathrm{C}\right)$ in einer Spirale zuerst durch das Heizungswasser des Pufferspeichers mit dem niedrigsten Temperaturniveau strömt, bevor die Brauchwassertemperatur im nachfolgenden Pufferspeicher weiter angehoben wird. Im Anschluss hebt das konventionelle Speicherladesystem der Heizungsanlage das Brauchwasser auf die für den Legionellenschutz vorgeschriebene Temperatur von $60^{\circ} \mathrm{C}$ an [20]. 


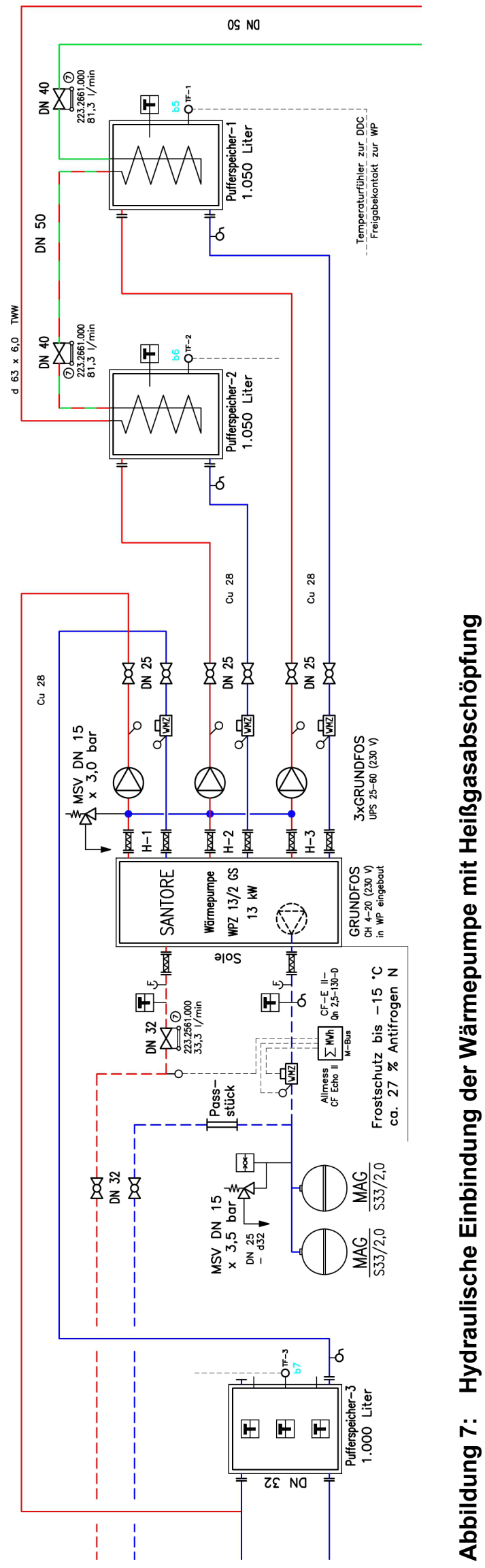


Die konventionelle Heizungsanlage der Jugendherberge besteht aus einer zentralen Pumpenwarmwasserheizung mit Radiatoren (Auslegung $70 / 50{ }^{\circ} \mathrm{C}$ ) und einem Speicherladesystem zur Brauchwassererwärmung (ThermoDualS-1000-77, FWT Wärmetechnik AG, Hamburg, Deutschland). Die benötigte Wärmemenge wird von einem 225 kW Gas-Brennwertkessel (Vitocrossal 300 Typ CT3, Viessmann Werke GmbH \& Co KG, Allendorf, Deutschland) bereitgestellt. Die Wärmeverteilung im Heizungsraum erfolgt über sechs Heizgruppen. Das Speicherladesystem setzt sich aus einem 1000 I Brauchwasserspeicher mit einem $130 \mathrm{~kW}$ Plattenwärmetauscher zusammen. Neben der vorgeschriebenen thermischen Legionellenprophylaxe wird dem Brauchwasser zusätzlich ein chemischer Zusatzstoff über eine Dosiervorrichtung (Berkoin AW 2-45, Berkados AM 3-40-V, VWS Deutschland GmbH, Celle, Deutschland) zugemischt.

Die Regelung der Stauwärmenutzungsanlage wird ausschließlich durch die Wärmepumpenregelung gesteuert (Abb. 8). Für die Steuerung der Wärmepumpe wurde jeweils ein Temperaturfühler in dem kältesten Pufferspeicher zur Brauchwasservorerwärmung und in den Rücklauf der Soleleitung installiert. Über diese beiden Temperaturfühler steuert die Wärmepumpenregelung den Wärmepumpenbetrieb. Für einen Wärmepumpenbetrieb wurde eine Mindest-Rücklauftemperatur der Sole von $10^{\circ} \mathrm{C}$ festgelegt. Auf der warmen Seite der Wärmepumpe wurde eine Pufferspeichertemperatur von $30{ }^{\circ} \mathrm{C}$ vorgegeben. Diese beiden Parameter wurden ausgewählt, um eine möglichst hohe Arbeitszahl zu erzielen. Die Ventilatoren wurden in den Regelkreislauf der Wärmepumpe integriert und laufen bei einer Anforderung aus dem System mit den Sole- und Heizungsumwälzpumpen an. Neben der elektronischen Wärmepumpenregelung reguliert ein eingebautes MOPExpansionsventil (MOP, Maximum Operating Pressure) bei zu hohen Soletemperaturen den Druck der durchströmenden Sole im Wärmetauscher, so dass der Wärmepumpenbetrieb auf Grund zu hoher Soletemperaturen nicht eingestellt werden muss.

Die konventionelle Heizungsanlage der Jugendherberge besitzt eine eigenständige Heizungsregelung (DDC 3000, Kieback \& Peter GmbH \& Co. 
KG, Berlin, Deutschland). Durch ein Steuersignal ist die Wärmepumpe mit der Heizungsregelung der konventionellen Heizungsanlage verbunden und im Regelablauf des Heizungssystems vor den Heizungskessel geschaltet (Abb. 8). Der Kessel wartet generell drei Minuten auf ein Signal von der Wärmepumpe und schaltet sich nur ein, wenn die Wärmepumpe aufgrund eines nicht ausreichenden Wärmeangebotes oder einer Störung kein Signal sendet. Schafft die Wärmepumpe innerhalb einer Stunde nicht, die Heizungsanforderung aus dem System zu bedienen, wird der Kessel freigegeben. Weiterhin erfasst die konventionelle Heizungsregelung die Temperaturen in den Pufferspeichern zur Brauchwasservorerwärmung und dem Heizungspufferspeicher, um die von der Stauwärmenutzungsanlage bereitgestellte Energie sinnvoll in das Heizungssystem einbinden zu können. Die Regelungstechnik des konventionellen Heizungssystems wurde weiterhin über eine Modemverbindung mit der Gebäudeleittechnik (GLT, Kieback \& Peter GmbH \& Co. KG, Berlin, Deutschland) der Universität Göttingen verbunden. Mit Hilfe der Gebäudeleittechnik ist es möglich, im Bedarfsfall von Göttingen aus in das konventionelle Heizungssystem einzugreifen und Trendkurven abzufragen. 


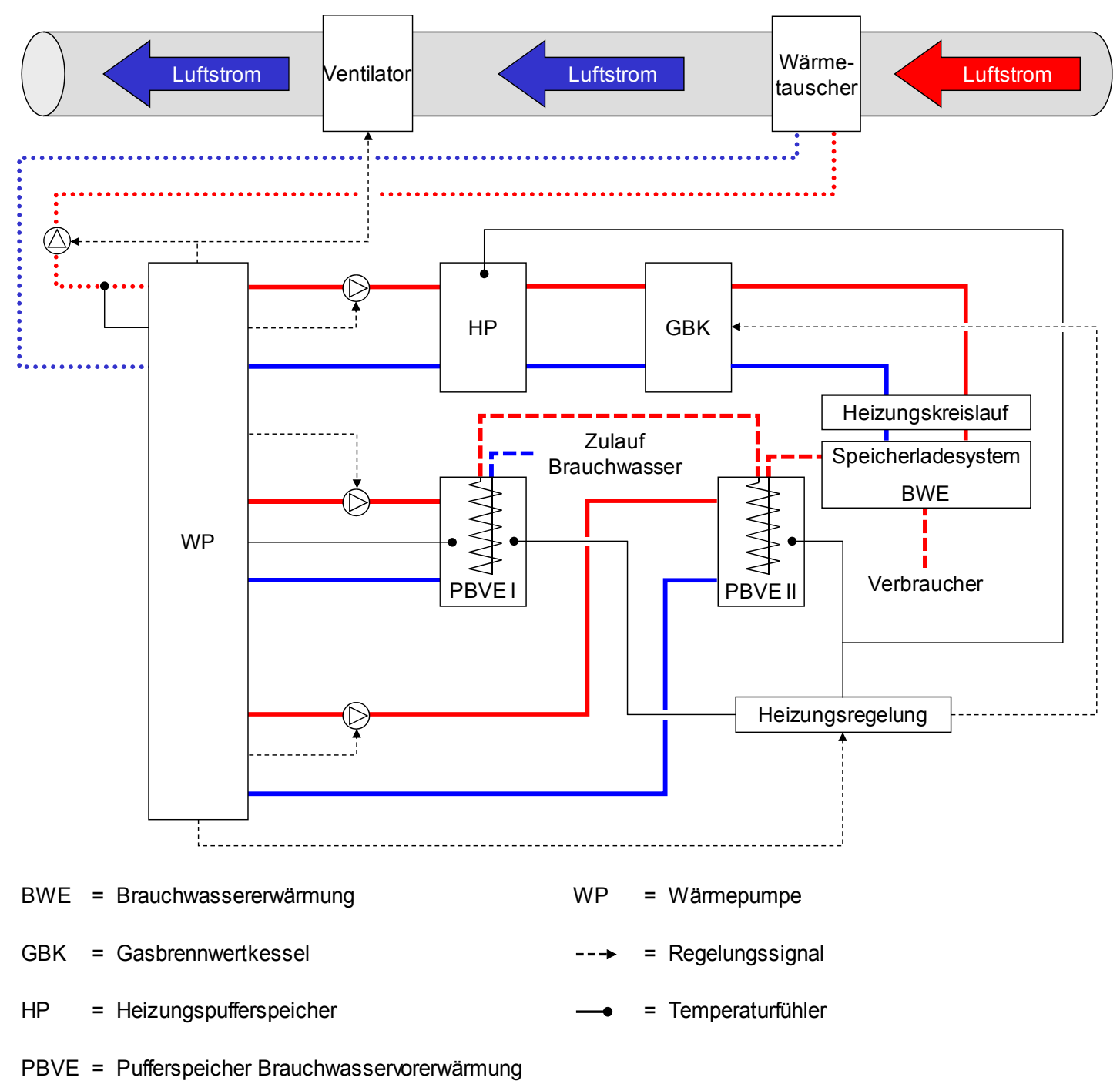

Abbildung 8: Einbindung in den Heizungsregelungsprozess

\subsection{Messtechnische Gestaltung}

\subsubsection{Dachraum}

Zur Messung des Strahlungsangebotes wurde ein Pyranometer (Pyranometer CM 6 B, Kipp \& Zonen B. V., Delft, Niederlande) in der Mitte der genutzten Dachfläche montiert. Die für die Temperaturmessungen verwendeten Widerstandsthermometer (W-SZK (0) PT 100, Klasse B, Heraeus Holding $\mathrm{GmbH}$, Hanau, Deutschland) wurden in einem Eis-Wärme-Bad mit einer Messgenauigkeit von $\pm 0,2 \mathrm{~K}$ kalibriert. Für die Ermittlung der Temperaturänderung durch den Solardach-Luft-Kollektor wurden im 
Strömungskanal des Daches die Temperaturen der durchströmenden Luft ermittelt (Abb. 9). Die Lufteintrittstemperatur wurde für jedes Kollektorfeld unmittelbar hinter dem Insektenschutzgitter erfasst. Weitere Messungen erfolgten in den jeweiligen Luftsammeltrichtern an der Luftaustrittseite der Kollektoren. Kurz vor dem Wärmetauscher wurde die Lufteintrittstemperatur in den Wärmetauscher gemessen. Die Luftaustrittstemperatur aus dem Wärmetauscher wurde mit jeweils einem Widerstandsthermometer pro Rohrleitung erfasst.

Die Raumtemperatur im Dachboden wurde durch ein zusätzliches Widerstandsthermometer aufgezeichnet, um auf mögliche Einflüsse des Wärmeangebotes im Dachraum und der Stauwärmenutzung schließen zu können.

Um eine Verfälschung der Messergebnisse durch stehende Luft im Solardach-Luft-Kollektor während der Untersuchungen zu vermeiden, wurden die Ventilatoren im Dauerbetrieb gefahren und lediglich die von der Wärmepumpensteuerung übermittelten theoretischen Ein- und Ausschaltzeiten für spätere Berechnungen registriert.

Für die Messung des Luftvolumenstroms wurden zwei Venturidüsen (KurzVenturirohr MVR 200, Druck-Messumformer Peritact 2000-K, Arthur Grillo $\mathrm{GmbH}$, Ratingen, Deutschland) zur Volumenstrommessung verwendet (Abb. 9). Die Venturidüsen wurden nach den für Messungen nach dem Venturi-Prinzip vorgeschriebenen Beruhigungslängen zwischen dem Wärmetauscher und den Ventilatoren eingebaut [15].

Die elektrische Leistungsaufnahme der Gebläse wurde mit einem Leistungsmessgerät erfasst (OC35-W Wattumformer, Orbit Controls AG, Schlieren, Schweiz). Die installierten Messgeräte aus dem Dachraum wurden über ein Datenlogger-System (ADL-MX Advanced Datalogger MX, ADL-MXE Advanced Datalogger Extension, e.bloxx A3-1, Meier-NT, Zwönitz, Deutschland) abgefragt. Die Messwertaufnahme im Dachraum ist in Abbildung 9 schematisch mit den einzelnen Messpunkten dargestellt. 


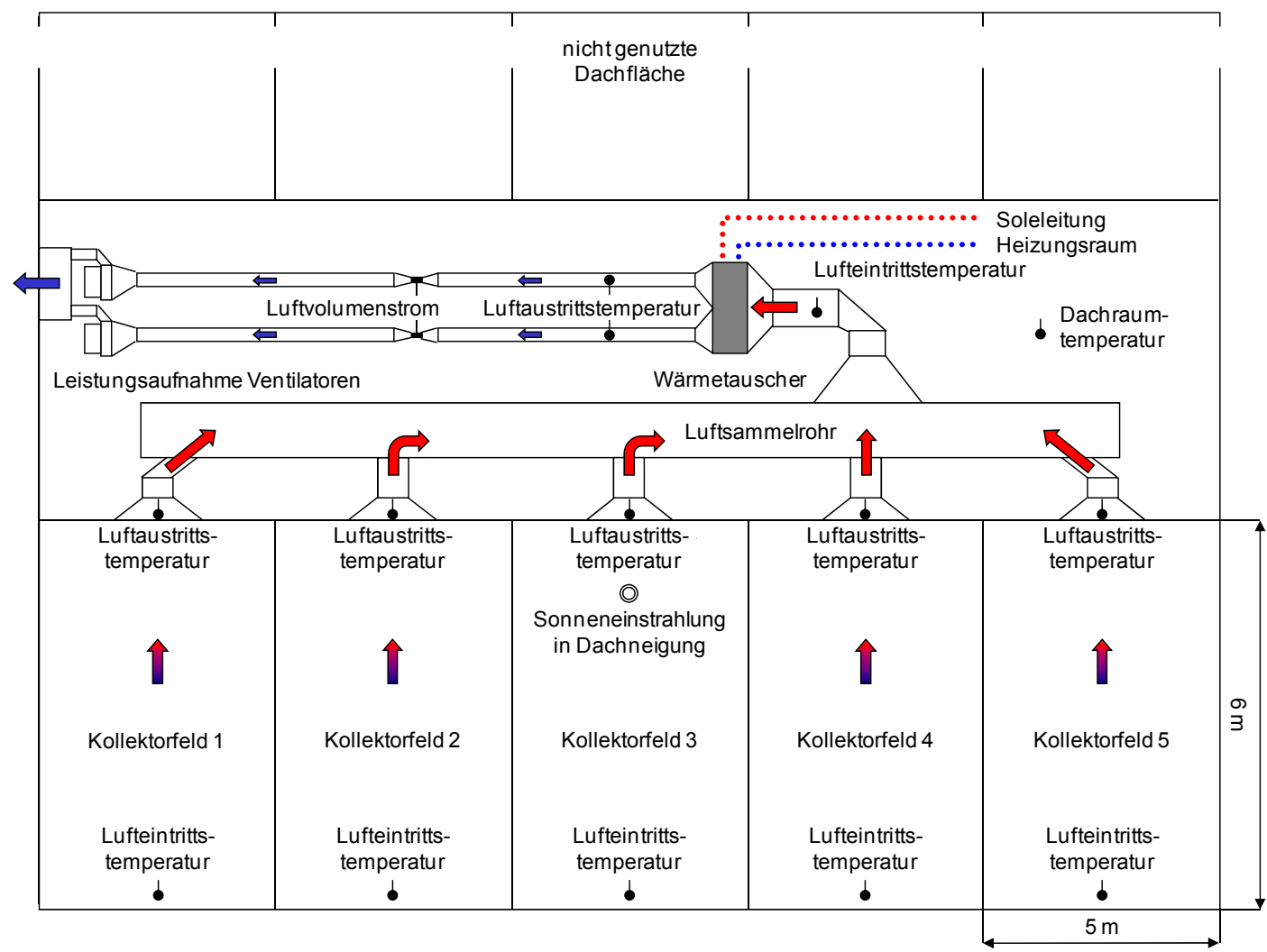

Abbildung 9: Schematische Darstellung der Messwertaufnahme im Dachraum/Dach

\subsubsection{Heizungsraum}

Zur Ermittlung der bereitgestellten Energie durch den Wärmetauscher wurde ein Wärmemengenzähler (CF-Echo II, Allmess GmbH, Oldenburg, Deutschland) auf der Soleseite der Wärmepumpe eingebaut. Auf der Wasserseite der Wärmepumpe wurde für jeden Heizkreislauf je ein Wärmemengenzähler installiert. In Verbindung mit der elektrischen Leistungsaufnahme kann anhand dieser Wärmemengenzähler die Leistungsziffer bzw. Arbeitszahl der Wärmepumpe berechnet werden. Ein weiterer Wärmemengenzähler erfasst den gesamten Wärmebedarf der Jugendherberge. Dieser wird benötigt, um den Anteil der Stauwärmenutzungsanlage am Gesamtwärmebedarf der Jugendherberge zu berechnen. Für die Ermittlung der elektrischen Leistungsaufnahme der Wärmepumpe, Sole- und Umwälzpumpen wurde ein Leistungsmessgerät (Kamstrup 382, Kamstrup A/S, Mannheim, Deutschland) installiert. Die installierten Messgeräte im 
Heizungsraum sind alle M-Bus-fähig (M-Bus, Meter-Bus) und werden über eine Zentraleinheit (M-Bus Mini ZE 20, Allmess $\mathrm{GmbH}$, Oldenburg, Deutschland) abgefragt. Die einzelnen Messpunkte im Heizungsraum sind in Abbildung 10 schematisch dargestellt.

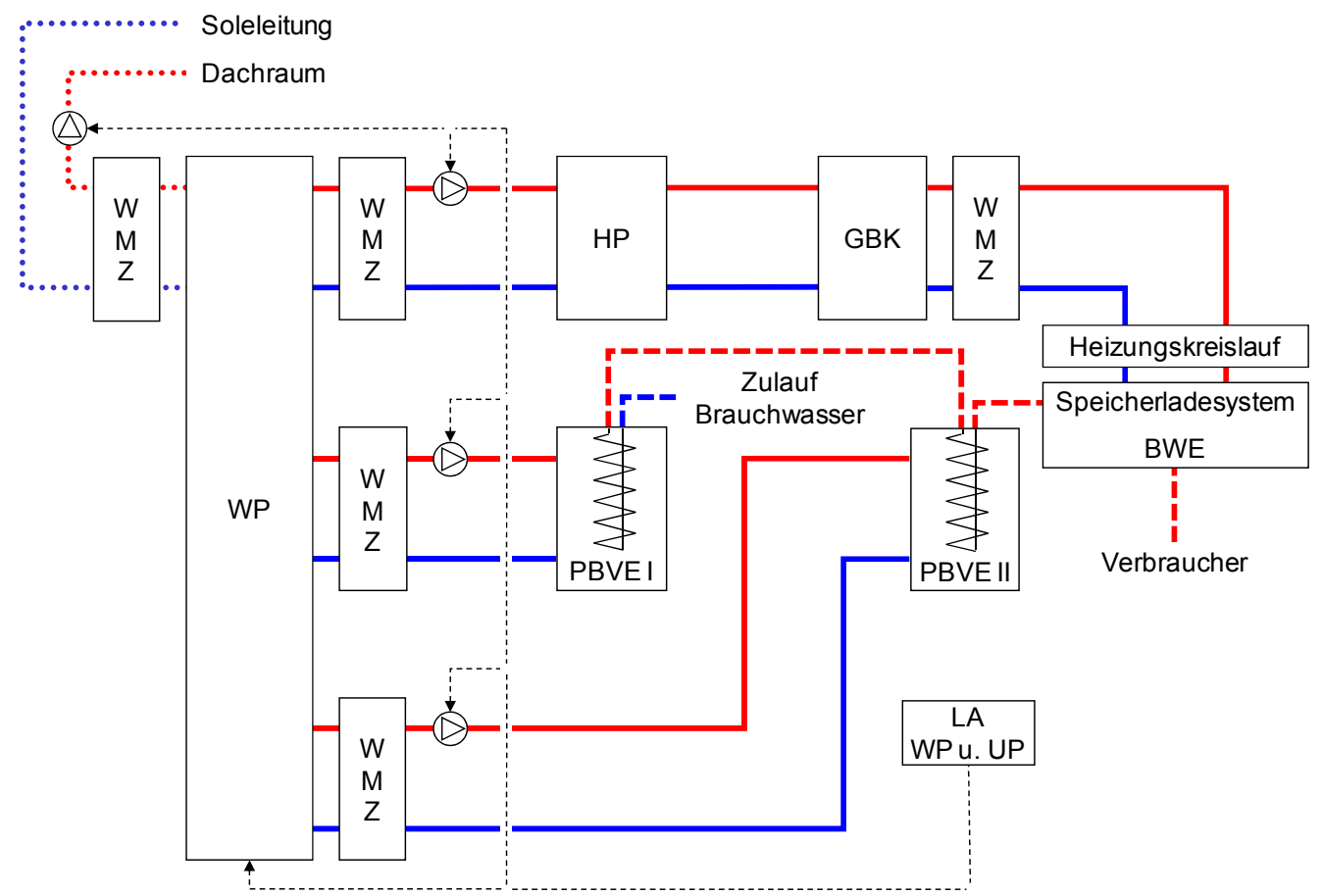

$$
\begin{array}{ll}
\text { BWE }=\text { Brauchwassererwärmung } & \text { PBVE }=\text { Pufferspeicher Brauchwasservorerwärmung } \\
\text { GBK }=\text { Gasbrennwertkessel } & \text { UP }=\text { Umwälzpumpen } \\
\mathrm{HP}=\text { Heizungspufferspeicher } & \text { WMZ }=\text { Wärmemengenzähler } \\
\text { LA }=\text { Leistungsaufname } & \text { WP }=\text { Wärmepumpe }
\end{array}
$$

Abbildung 10: Schematische Darstellung der Messwertaufnahme im Heizungsraum 


\subsubsection{Wetterstation}

Neben der eigenen Erfassung des Globalstrahlungsangebotes und der Lufteintrittstemperatur in den Kollektor wurden zusätzlich meteorologische Daten von einer Wetterstation (Meteomedia Messstation Variante 4, Adolf Thies $\mathrm{GmbH}$ \& Co. KG, Göttingen, Deutschland) aufgezeichnet. Die Messung der meteorologischen Daten orientierte sich an den Vorgaben der Meteomedia AG. Ziel war es, die Vergleichbarkeit zu anderen MeteomediaMessstationen in Deutschland herzustellen, um anhand der gewonnenen Erkenntnisse der Pilotanlage zukünftig auch Aussagen über andere Standorte in Deutschland treffen zu können.

Die Messstationen wurden aus versuchstechnischen Gründen in eine Dachund Bodenstation aufgeteilt. Die Dachstation registrierte über einen am Gebäude befestigten Messmast Globalstrahlung, Sonnenscheindauer, Windrichtung und Windgeschwindigkeit. Durch die Bodenstation wurden Lufttemperatur, Erdoberflächentemperatur, relative Luftfeuchte, Niederschlagssumme und der barometrische Luftdruck erfasst.

\subsubsection{Technikraum}

Für die automatische Erfassung der Messdaten wurden zwei Prozessrechner im Technikraum der Jugendherberge mit Internetzugang installiert. Ein Rechner speicherte die Messwerte der Abteilung Agrartechnik und der zweite Rechner die meteorologischen Daten der Meteomedia AG Wetterstation. Der Prozessrechner der Abteilung Agrartechnik ist mit dem Datenlogger - System aus dem Dachraum und der Zentraleinheit aus dem Heizungsraum verbunden und speichert die abgefragten Messwerte. Mit Hilfe einer LAN Schnittstelle (LAN, Local Area Network) wurden die nicht selbst aufgezeichneten meteorologischen Daten der Wetterstation auf den Prozessrechner der Abteilung Agrartechnik übertragen. Ein FTP - Programm (FTP, File Transfer Protocol) sendete die gespeicherten Daten automatisch über eine Internetverbindung auf den Server der Abteilung Agrartechnik in 
Göttingen (Abb. 11). Die Prozessrechner wurden neben der Messdatenerfassung für die Darstellung aktueller Messwerte des Energiedaches sowie für die Illustration meteorologischer Daten der Wetterstation in der Jugendherberge genutzt.

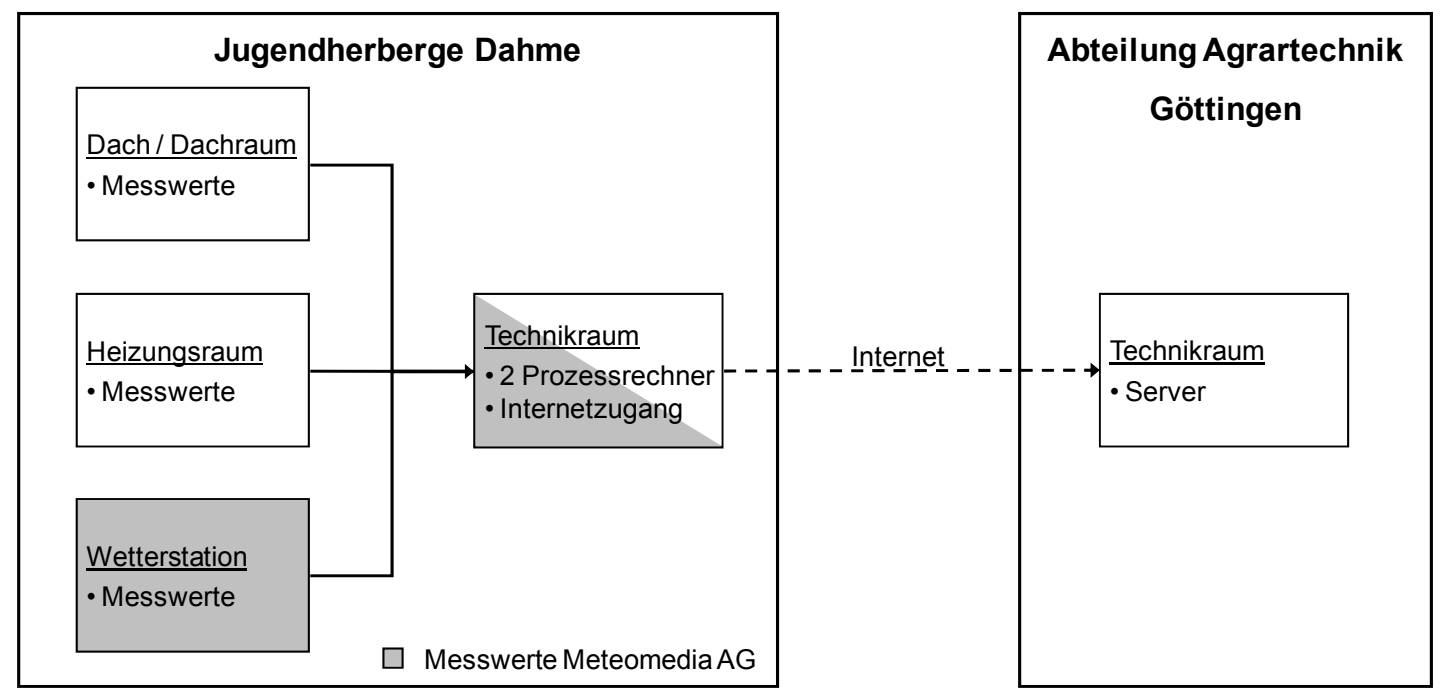

Abbildung 11: Übersicht der Messdatenerfassung

\subsection{Durchführung der Versuche}

Die Versuche fanden in dem Zeitraum von Dezember 2008 bis November 2009 statt. Es wurden Versuche zum Betriebsverhalten der Gesamtanlage und zur Bestimmung möglicher Einflussfaktoren auf den geförderten Luftvolumenstrom durchgeführt, die im Folgenden weiter beschrieben werden.

\section{Kontinuierliche Aufzeichnung des Betriebsverhaltens der Gesamtanlage}

Für die Bestimmung des Betriebsverhaltens der Stauwärmenutzungsanlage wurden sämtliche Messwerte der Stauwärmenutzungsanlage mit dem Computerprogramm TestPoint 3.4 (TestPoint 3.4, Capital Equipment Corp (CEC), Bedford, USA) abgefragt und in einem Abstand von zwei Minuten gespeichert. Eine höhere Speicherrate war aufgrund der Vielzahl der Messwerte technisch nicht möglich. Für die Globalstrahlung und Windstärke 
wurde aufgrund der großen Schwankungsbreite der errechnete Mittelwert über zwei Minuten gespeichert. Zusätzlich wurden für die Windstärke die minimalen und maximalen Messwerte registriert. Diese Messwerte wurden dann mit den übrigen Momentanmesswerten aus dem Dachraum, der Wetterstation und dem Heizungsraum in zwei zeitlich synchronisierte Datendateien gespeichert. Dies war notwendig, da das Messprogramm nur eine begrenzte Anzahl von Messwerten in einer Datei speichern konnte.

\section{Bestimmung der Einflussfaktoren auf den geförderten Luftvolumenstrom}

Neben der kontinuierlichen Messwertaufnahme wurden zusätzlich zwei zeitlich voneinander getrennte Versuche für die Bestimmung der Einflussfaktoren auf den geförderten Luftvolumenstrom $\left(\dot{V}_{L}\right)$ durchgeführt. Ziel dieser Versuche war es, herauszufinden, wie stark die einzelnen „statischen“ Faktoren der Pilotanlage den Luftvolumenstrom beeinflussen.

Folgende Faktoren wurden untersucht:

- Einfluss der Kollektorfelder (KF)

- Einfluss des Wärmetauschers (WT)

- Einfluss des Rohrleitungssystems (RS)

- Einfluss des Insektenschutzgitters (ISG)

Im ersten Versuch wurde der Einfluss der Kollektorfelder (KF) auf den Luftvolumenstrom $\left(\dot{V}_{L}\right)$ untersucht. Hierzu wurden die einzelnen Kollektorfelder nacheinander geschlossen, bis alle möglichen Kollektorfeldkombinationen getestet waren. Für jeden Betriebszustand wurde der Luftvolumenstrom an den Digitalanzeigen der zwei Venturidüsen abgelesen und zusammen mit der Lufttemperatur $\left(t_{L}\right)$ protokolliert. 
Im zweiten Versuch wurden der Luftvolumenstrom $\left(\dot{V}_{L}\right)$ und die Lufttemperatur $\left(t_{L}\right)$ für den Betriebszustand „alle Kollektorfelder geöffnet“ und „nur Kollektorfeld 5 geöffnet “als Referenzwert vermerkt. Im Anschluss wurde der Einfluss des Insektenschutzgitters (ISG) auf den geförderten Luftvolumenstrom untersucht. Für diesen Versuchsabschnitt wurde das Insektenschutzgitter des Kollektorfeldes 5 (Abb. 9) auf einer Länge von $62 \mathrm{~cm}$ um $5 \mathrm{~cm}$ geöffnet und die Messwerte wurden für den Betriebszustand „alle Kollektorfelder geöffnet“ registriert. Dies wurde ebenfalls für den Betriebszustand „nur Kollektorfeld 5 geöffnet“ wiederholt. Dieser Versuch konnte aufgrund der baulichen Gegebenheiten nur an Kollektorfeld 5 durchgeführt werden, da die übrigen Insektenschutzgitter der einzelnen Kollektorfelder nicht zugänglich waren.

Für die Bestimmung des Einflusses des Solardach-Luft-Kollektors auf den Luftvolumenstrom wurde das Rohrleitungssystem (RS) nach dem Wärmetauscher (WT) abgetrennt und die Messwerte wurden protokolliert. Die Luft wurde in diesem Versuchsabschnitt lediglich aus dem Dachraum über die runde Rohraufnahmeöffnung des Trichters angesaugt. Die Abnahme des Trichters gestaltete sich schwierig, da dieser mit dem Wärmetauschergehäuse verschraubt und verklebt war. Aus diesem Grund wurde der Trichter in diesem Versuchsabschnitt nicht entfernt, da dies nicht ohne Beschädigung der Bauteile möglich gewesen wäre.

In der letzten Versuchsanordnung wurde das Rohrleitungssystem vor dem Wärmetauscher abgetrennt, um auf den Einfluss des Wärmetauschers und des verwendeten Rohrleitungssystems nach dem Wärmetauscher schließen zu können. Hierzu wurde ebenfalls die Luft aus dem Dachraum angesaugt und die Messwerte wurden notiert. 


\subsection{Versuchsauswertung}

Die jetzt bestehende Pilotanlage ist in mehreren Entwicklungsstufen entstanden. Die Auswertung der aufgezeichneten Messwerte der Stauwärmenutzungsanlage ist aus diesem Grund nur für den Solardachluftkollektor möglich. Für eine erste Einschätzung der gesamten Stauwärmenutzungsanlage wurden die Messwerte des Solardachluftkollektors anhand von Simulationsrechnungen auf das dahinter stehende Nutzungssystem bezogen. Für die Versuchsauswertung wurden deshalb nur die Messwerte aus dem Dachraum und der Messwert des Gesamtwärmemengenzählers aus dem Heizungsraum herangezogen. Von der meteorologischen Wetterstation wurden ausschließlich die Messwerte der relativen Luftfeuchte, Lufttemperatur und Windgeschwindigkeit für die Ergebnisauswertung verwendet. Die Datensätze wurden in das Datenbankprogramm Microsoft Offices Access 2007 eingelesen und auf ihre Plausibilität überprüft. Datensätze mit nicht plausiblen Messwerten wurden gelöscht. Die nicht plausiblen Messwerte lagen unter einem Prozent und wurden in den folgenden Auswertungen nicht weiter berücksichtigt. Tage mit größeren Datensatzlücken, die durch Stromausfälle entstanden sind, wurden gelöscht. Für fehlende Tagesdatensätze wurde der Mittelwert aus den vorhandenen Tagesdatensätzen innerhalb eines Monates gebildet und eingesetzt.

Die Messwerte der Volumenstrommessungen $\left(\dot{V}_{L}\right)$ wurden anhand der gemessenen Luftemperaturen $\left(t_{L}\right)$ in den beiden Rohrleitungen vor dem Wärmetauscher nach der Formel (1) korrigiert [53]. Dies ist notwendig, da der Messwertumformer werkseitig auf eine Luftemperatur von $30^{\circ} \mathrm{C}$ kalibriert wurde. 
$I_{A}=I_{A_{\left(30^{\circ} \mathrm{C}\right)}} \sqrt{\frac{303}{273+t_{L}}}$

Darin sind:

$\mathrm{I}_{\mathrm{A}} \quad=$ Ausgangsstrom $\quad[\mathrm{A}]$

$\mathrm{I}_{\mathrm{A}_{\left(30^{\circ} \mathrm{C}\right)}}=$ Ausgangsstrom bei $30^{\circ} \mathrm{C} \quad[\mathrm{A}]$

$\mathrm{t}_{\mathrm{L}}=$ Lufttemperatur $\quad\left[{ }^{\circ} \mathrm{C}\right]$

Um den Einfluss des jahreszeitlichen Temperaturverlaufes herauszuarbeiten, wurde der Jahresdatensatz für die Auswertungen den meteorologischen Jahreszeiten zugeordnet.

Weiterhin wurden die Tage anhand des höchsten Globalstrahlungswertes in den Mittagsstunden in eine erste und zweite Tageshälfte geteilt, um mögliche Unterschiede zwischen den Tageshälften identifizieren zu können.

Die Berechnung der Temperaturdifferenz $(\Delta \mathrm{T})$ zwischen der Kollektoraustrittsluft $\left(\mathrm{T}_{\mathrm{a}}\right)$ und der Kollektoreintrittsluft $\left(\mathrm{T}_{\mathrm{e}}\right)$ wurde anhand der Formel (2) berechnet [35].

$\Delta \mathrm{T}=\mathrm{T}_{\mathrm{a}}-\mathrm{T}_{\mathrm{e}}$

Darin sind:

$$
\begin{array}{lll}
\Delta \mathrm{T} & =\text { Temperaturdifferenz } & {[\mathrm{K}]} \\
\mathrm{T}_{\mathrm{a}} & =\text { Austrittstemperatur } & {[\mathrm{K}]} \\
\mathrm{T}_{\mathrm{e}} & =\text { Eintrittstemperatur } & {[\mathrm{K}]}
\end{array}
$$


Der Kollektorwirkungsgrad $(\eta)$ ist definiert als das Verhältnis zwischen der Nutzleistung und der in Kollektorebene eingestrahlten Globalstrahlung $[35,60]$. Für die Berechnung des Kollektorwirkungsgrades wurde zunächst die Kollektorfläche (A ) nach Formel (3) bestimmt.
$\mathrm{A}=1 \cdot \mathrm{b}$

Darin sind:
$\mathrm{A}=$ Fläche $\left[\mathrm{m}^{2}\right]$
$1=$ Länge $[\mathrm{m}]$
$\mathrm{b}=$ Breite $[\mathrm{m}]$

Wirkungsgradberechnungen wurden nur für positive Temperaturdifferenzen zwischen der Kollektoraustrittsluft und der Kollektoreintrittsluft nach Formel (4) durchgeführt [25, 35, 61]. 


$$
\eta=\frac{\dot{\mathrm{m}}_{\mathrm{L}} \cdot \mathrm{c}_{\mathrm{pL}}\left(\mathrm{T}_{\mathrm{a}}-\mathrm{T}_{\mathrm{e}}\right)}{\mathrm{A} \cdot \dot{\mathrm{G}}_{\mathrm{g}}}
$$

Darin sind:

$$
\begin{array}{lll}
\eta & \text { Wirkungsgrad } & {[-]} \\
\dot{\mathrm{m}}_{\mathrm{L}}=\text { Massenstrom der Luft } & {[\mathrm{kg} / \mathrm{s}]} \\
\mathrm{c}_{\mathrm{pL}}=\text { spezifische Wärmekapazität der Luft } & {[1,005 \mathrm{~kJ} /(\mathrm{kg} \cdot \mathrm{K})]} \\
\mathrm{T}_{\mathrm{a}}=\text { Austrittstemperatur } & {[\mathrm{K}]} \\
\mathrm{T}_{\mathrm{e}}=\text { Eintrittstemperatur } & {[\mathrm{K}]} \\
\mathrm{A}=\text { Kollektorfläche } & {\left[\mathrm{m}^{2}\right]} \\
\dot{\mathrm{G}}_{\mathrm{g}}=\text { Globalstrahlung in der Kollektorebene } & {\left[\mathrm{kW} / \mathrm{m}^{2}\right]}
\end{array}
$$

Zur Bestimmung der möglichen Einflussfaktoren auf den Luftvolumenstrom wurde für jede Versuchsgruppe der Mittelwert (MW) gebildet und die Standardabweichung (STAW) der Messwerte berechnet. Für die Berechnung der Luftgeschwindigkeiten $\left(\mathrm{V}_{\mathrm{L}}\right)$ in den einzelnen Anlagenabschnitten wurden zunächst die rechteckigen Querschnittsflächen $\left(A_{r}\right)$ nach Formel (5) bestimmt.

$$
\mathrm{A}_{\mathrm{r}}=\mathrm{h} \cdot \mathrm{b}
$$

Darin sind:
$\mathrm{A}_{\mathrm{r}} \quad=$ rechteckige Querschnittsfläche $\left[\mathrm{m}^{2}\right]$
$\mathrm{h}=$ Höhe
[m]
$\mathrm{b}=$ Breite
[m] 
Die durch die Dachlattung und Wärmetauscherlamellen verursachten Querschnittsverengungen wurden nicht berücksichtigt. Nach Formel (6) wurde die Rohrquerschnittsfläche $\left(A_{R}\right)$ berechnet [26].

$$
\mathrm{A}_{\mathrm{R}}=\pi \frac{\mathrm{d}^{2}}{4}
$$

Darin sind:
$A_{R}=$ Rohrquerschnittsfläche $\left[\mathrm{m}^{2}\right]$
$\pi \quad=\mathrm{Pi}$
$[3,141592654]$
$\mathrm{d} \quad=$ Rohrdurchmesser
$[\mathrm{m}]$

Nach der Berechnung der Lufteintrittsflächen wurden die Luftgeschwindigkeiten für die einzelnen Bauteile nach Formel (7) berechnet [26, 76].

$$
\mathrm{v}_{\mathrm{L}}=\frac{\dot{\mathrm{V}}_{\mathrm{L}}}{\mathrm{A}_{\mathrm{Q}}}
$$

Darin sind:

$$
\begin{array}{ll}
\mathrm{V}_{\mathrm{L}}=\text { Luftgeschwindigkeit } & {[\mathrm{m} / \mathrm{s}]} \\
\dot{\mathrm{V}}_{\mathrm{L}}=\text { spezifischer Luftvolumenstrom } & {\left[\mathrm{m}^{3} / \mathrm{s}\right]} \\
\mathrm{A}_{\mathrm{Q}}=\text { Querschnittsfläche } & {\left[\mathrm{m}^{2}\right]}
\end{array}
$$

Nachdem zunächst der Solardach-Luft-Kollektor ohne das dahinter stehende Nutzungssystem ausgewertet wurde, wird der Kollektor im Folgenden mit dem dahinter stehenden Nutzungssystem in Beziehung gebracht. Zunächst wurde die abgegebene thermische Energie $\left(\mathrm{E}_{\text {th }}\right)$ an den Wärmetauscher berechnet. Für die Berechnung wurde zuerst der Sättigungsdampfdruck $\left(\mathrm{p}_{\mathrm{s}}\right)$ 
mit der vereinfachten Approximationsformel (8) nach Glück (1991) bestimmt [33].

$\mathrm{p}_{\mathrm{s}}=611 \exp \left(-1,91275 \cdot 10^{-4}+7,258 \cdot 10^{-2} \mathrm{t}_{\mathrm{L}}-2,939 \cdot 10^{-4} \mathrm{t}_{\mathrm{L}}{ }^{2}+\right.$

$\left.9,841 \cdot 10^{-7} \mathrm{t}_{\mathrm{L}}^{3}-1,92 \cdot 10^{-9} \mathrm{t}_{\mathrm{L}}{ }^{4}\right)$ in Pa

Darin sind:

$$
\begin{array}{ll}
\mathrm{p}_{\mathrm{s}}=\text { Sättigungsdampfdruck } & {[\mathrm{Pa}]} \\
\mathrm{t}_{\mathrm{L}}=\text { Lufttemperatur } & {\left[{ }^{\circ} \mathrm{C}\right]}
\end{array}
$$

Nach Formel (9) lässt sich im Anschluss der Feuchtegehalt ( $\left.\mathrm{x}_{\mathrm{L}}\right)$ der Luft berechnen [58].

$$
\mathrm{x}_{\mathrm{L}}=0,622 \frac{\mathrm{kg}}{\mathrm{kg}} \frac{\varphi \cdot \mathrm{p}_{\mathrm{s}}}{\mathrm{p}_{\mathrm{L}}-\varphi \cdot \mathrm{p}_{\mathrm{s}}}
$$

Darin sind:

$$
\begin{aligned}
& x_{L}=\text { Feuchtegehalt der Luft } \quad\left[\mathrm{kg}_{\mathrm{H}_{2} \mathrm{O}} / \mathrm{kg}_{\text {Luft }}\right] \\
& \varphi \quad=\text { relative Luftfeuchtigkeit }[-] \\
& \mathrm{p}_{\mathrm{s}}=\text { Sättigungsdampfdruck }[\mathrm{Pa}] \\
& \mathrm{p}_{\mathrm{L}}=\text { barometrischer Luftdruck }[\mathrm{Pa}]
\end{aligned}
$$

Mit der Formel (10) wurden die Enthalpien der Luft $\left(\mathrm{h}_{1+\mathrm{x}}\right)$ vor und nach dem Wärmetauscher berechnet [30,63]. Für die Berechnung der Austrittsenthalpie $\left(\mathrm{h}_{1+\mathrm{x}_{\text {Aus }}}\right)$ wurde die Luftaustrittstemperatur (LAT) am Wärmetauscher auf $10^{\circ} \mathrm{C}$ festgelegt, da die Wärmepumpentechnik die Lufttemperatur aufgrund des verwendeten Wärmetauschers auf maximal $10^{\circ} \mathrm{C}$ abkühlen kann. 
Es gelten folgende Annahmen:

$\mathrm{h}=0$ für trockene Luft von $0^{\circ} \mathrm{C}$

$\mathrm{h}=0$ für flüssiges Wasser bei $0^{\circ} \mathrm{C}$

Enthalpie des ungesättigten Gemisches $(\varphi<1)$

$\mathrm{h}_{1+\mathrm{x}}=\mathrm{c}_{\mathrm{pL}}^{0} \mathrm{t}_{\mathrm{L}}+\mathrm{x}_{\mathrm{L}}\left(\Delta \mathrm{h}_{\mathrm{v} 0}+\mathrm{c}_{\mathrm{pD}}^{0} \mathrm{t}_{\mathrm{L}}\right)$

Darin sind:

$\mathrm{h}_{1+\mathrm{x}}=$ Enthalpie des ungesättigten Gemisches

$[\mathrm{kJ} / \mathrm{kg}]$

$\mathrm{c}_{\mathrm{pL}}^{0} \quad=\quad$ spezifische Wärmekapazität der trockenen Luft $\quad[1,007 \mathrm{~kJ} /(\mathrm{kg} \cdot \mathrm{K})]$

$\mathrm{t}_{\mathrm{L}} \quad=\quad$ Lufttemperatur

$\left[{ }^{\circ} \mathrm{C}\right]$

$\mathrm{x}_{\mathrm{L}}=$ Feuchtegehalt der Luft

$\left[\mathrm{kg}_{\mathrm{H}_{2} \mathrm{O}} \mathrm{kg}\right.$ Luft $]$

$\Delta \mathrm{h}_{\mathrm{v} 0}=$ Verdampfungsenthalpie bei $0{ }^{\circ} \mathrm{C}$

[2501 kJ/kg]

$\mathrm{c}_{\mathrm{pD}}^{0} \quad=$ spezifische Wärmekapazität Wasserdampf

$[1,865 \mathrm{~kJ} /(\mathrm{kg} \cdot \mathrm{K})]$

Nach der Berechnung der Ein- und Austrittsenthalpie wurde die abgegebene thermische Leistung $\left(\mathrm{P}_{\text {th }}\right)$ nach Formel (11) berechnet. 
$\mathrm{P}_{\text {th }}=\dot{\mathrm{m}}_{\mathrm{L}} \mathrm{h}_{1+\mathrm{x}_{\text {Ein }}}-\dot{\mathrm{m}}_{\mathrm{L}} \mathrm{h}_{1+\mathrm{x}_{\text {Aus }}}$

Darin sind:

$\mathrm{P}_{\mathrm{th}} \quad=$ abgegebene thermische Leistung $\quad[\mathrm{kW}]$

$\dot{\mathrm{m}}_{\mathrm{L}} \quad=$ Massenstrom der Luft $\quad[\mathrm{kg} / \mathrm{s}]$

$\mathrm{h}_{1+\mathrm{x}_{\text {Ein }}}=$ Eintrittsenthalpie der Luft $\quad[\mathrm{kJ} / \mathrm{kg}]$

$\mathrm{h}_{1+\mathrm{x}_{\mathrm{Aus}}}=$ Austrittsenthalpie der Luft $\quad[\mathrm{kJ} / \mathrm{kg}]$

Mit der Formel (12) wurde anschließend die abgegebene thermische Energie $\left(E_{\text {th }}\right)$ berechnet.

$\mathrm{E}_{\mathrm{th}}=\mathrm{P}_{\mathrm{th}} \cdot \mathrm{Z}$

Darin sind:

$\mathrm{E}_{\mathrm{th}}=$ abgegebene thermische Energie $\quad[\mathrm{kWh}]$

$\mathrm{P}_{\mathrm{th}}=$ abgegebene thermische Leistung $\quad[\mathrm{kW}]$

$\mathrm{z}=$ Messintervall

[h]

Die abgegebene thermische Energie des jeweiligen Betrachtungszeitraumes setzt sich aus der Summe der Einzelenergien aus Formel (12) zusammen. Die bereinigte Energie ist das Ergebnis der Subtraktion der Ventilatorantriebsenergie von der verfügbaren thermischen Energie der Luft. Unter der Nutzenergie ist die Energiemenge zu verstehen, die für einen Wärmepumpenbetrieb zur Verfügung steht (Abb. 12). Für den Wärmepumpenbetrieb wurde eine Mindestenergiemenge der Luft $>0,1 \mathrm{kWh}$ festgelegt. Dieser Vorgabe liegt eine zu erreichende Arbeitszahl $(\beta)$ von 
mindestens drei zugrunde. Der Mindestwert wurde über das Verhältnis der abgegebenen Wärmemenge (aWM) und der dafür benötigten elektrischen Antriebsenergie (eAE) ermittelt.

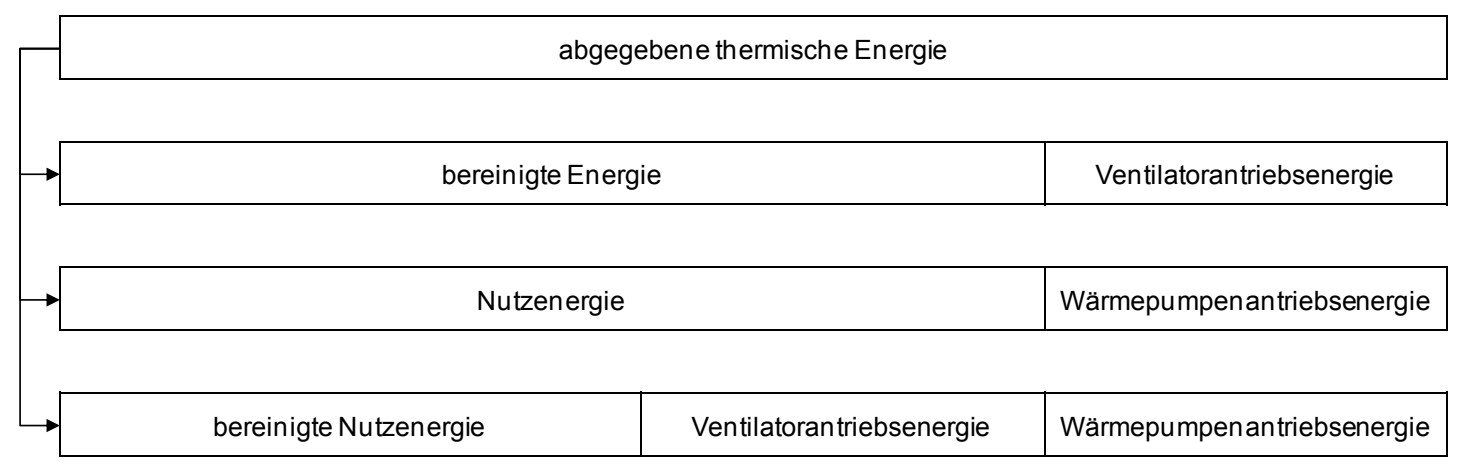

Abbildung 12: Definition der Energiemengen

Die in Abbildung 12 definierten Energiemengen gelten analog für die bereinigte Energie der Umgebungsluft, die bereinigte Nutzenergie der Umgebungsluft, die Kollektornutzenergie, die bereinigte Kollektornutzenergie und die bereinigte Gesamtnutzenergie. Für die Umgebungsluft ist bei der Betrachtung der Energien die mittlere Lufteintrittstemperatur in die Kollektorfelder angenommen worden. Die zusätzliche, durch den Kollektor gewonnene, Energie wurde durch Subtraktion des Energiegehaltes der Umgebungsluft von dem Energiegehalt der Kollektoraustrittsluft ermittelt. Der mögliche Nutzungszeitraum der Luft ergibt sich aus der Summe der Messwerte bei einem bestimmten Energiegehalt und der Multiplikation des Messintervalls $(z)$. Für eine effiziente Kollektornutzung (effiziente bereinigte Kollektornutzenergie) wurde unterstellt, dass der Energiegehalt zwischen der Kollektoreintrittsluft und der Kollektoraustrittsluft größer als die aufgewendete Antriebsenergie für die Ventilatoren ist. Ist dies nicht der Fall, soll Umgebungsluft über einen Bypass angesaugt werden, wenn der Energiegehalt der Umgebungsluft größer als die aufgewendete Antriebsenergie für die Ventilatoren ist (effiziente bereinigte Nutzenergie der Umgebungsluft). Die benötigte Antriebsenergie für die Ventilatoren wurde für das Ansaugen 
der Umgebungsluft aufgrund des verwendeten Rohrsystems gleich dem Kollektorbetrieb gesetzt.

Für die Bestimmung des thermischen Energiebedarfs der Jugendherberge wurden nur Messwerte des Gesamtwärmemengenzählers verwendet. Der anteilige Wärmebedarf $\left(\mathrm{E}_{\mathrm{W}}\right)$ für die Brauchwassererwärmung wurde nach Formel (13) berechnet [40]. Es wurde angenommen, dass das ankommende Brauchwasser eine Temperatur von $10^{\circ} \mathrm{C}$ besitzt und auf eine Temperatur von $40^{\circ} \mathrm{C}$ erwärmt wird. Als mittlerer Warmwasserverbrauch wurden 31 I pro Person und Tag unterstellt. Diese Annahmen wurden in Anlehnung an die Richtlinie VDI 2067 (VDI, Verein Deutscher Ingenieure e. V.) getroffen [72].

$$
\mathrm{E}_{\mathrm{w}}=\mathrm{m} \cdot \mathrm{c}_{\mathrm{pW}} \cdot \Delta \mathrm{T}
$$

Darin sind:

$$
\begin{array}{lll}
\mathrm{E}_{\mathrm{W}}=\text { Wärmebedarf Warmwasserbereitung } & {[\mathrm{Wh}]} \\
\mathrm{m}=\text { Masse } & {[\mathrm{kg} / \mathrm{d}]} \\
\mathrm{c}_{\mathrm{pW}}=\text { spezifische Wärmekapazität Wasser } & {[1,163 \mathrm{Wh} /(\mathrm{kg} \cdot \mathrm{K})]} \\
\Delta \mathrm{T}=\text { Temperaturdifferenz } & {[\mathrm{K}]}
\end{array}
$$

Anhand der Übernachtungszahlen (ÜN) der Jugendherberge wurde der monatliche Wärmebedarf für die Warmwasserbereitung der Jugendherberge ermittelt. Durch Subtraktion des Wärmebedarfes der Warmwasserbereitung von dem gemessenen Gesamtwärmebedarf der Jugendherberge wurde der Anteil des Heizungsbedarfs bestimmt. Der Heizungsbedarf beinhaltet, je nach Jahreszeit, sowohl den benötigten Energiebedarf für die Raumheizung als auch den Bedarf für die Aufrechterhaltung der Brauchwassertemperatur im Brauchwasserspeicher und der Zirkulationsleitung. 
Die Jahresarbeitszahl $\left(\beta_{\mathrm{a}}\right)$ ist nach der Richtlinie VDI 4650 „Das Verhältnis der im Jahr abgegebenen Nutzwärme bezogen auf die eingesetzte elektrische Energie für den Antrieb des Verdichters und der Hilfsantriebe“ [71]. Grundlage dieser Berechnung ist die Ermittlung der Leistungszahl ( $\varepsilon$ ) nach DIN EN 14511 und DIN EN 255-3 (DIN, Deutsches Institut für Normung e. V.; EN, Europäische Norm) [17, 18]. Da für die eingesetzte Wärmepumpe nur das technische Datenblatt des Verdichters zur Verfügung steht, wurde eine stark vereinfachte Berechnungsmethode angewendet [66]. Um die Leistungszahl $\left(\varepsilon_{\mathrm{V}}\right)$ der Wärmepumpe bestimmen zu können, mussten zunächst die Soletemperaturen berechnet werden. Hierzu wurden Mittelwerte von den gemessenen Lufttemperaturen nach dem Wärmetauscher, der gemessenen relativen Luftfeuchte (rLF) und dem gemessenen Luftvolumenstrom (LVS) für die einzelnen Jahreszeiten gebildet. In die Mittelwertbildung gingen nur Messwerte mit einer abgegebenen thermischen Energie $>0,1 \mathrm{kWh}$ (Kollektornutzenergie) ein. Dieser Vorgabe liegt wiederum eine zu erreichende Arbeitszahl ( $\beta$ ) von mindestens drei zugrunde. Der erforderliche Mindestwert wurde ebenfalls über das Verhältnis der abgegebenen Wärmemenge (aWM) der Wärmepumpe und der dafür benötigten elektrischen Antriebsenergie (eAE) ermittelt. Die Solevorlauftemperatur (SVT) wurde auf $6{ }^{\circ} \mathrm{C}$ mit einer Solespreizung (SSP) von mindestens $4 \mathrm{~K}$ festgelegt. Diese vorgegebenen Parameter resultieren aus der Auslegung des Wärmetauschers im Dachraum und wurden von dem Wärmepumpenhersteller vorgegeben [65]. Weiterhin wurde eine temperaturabhängige Steuerung des Solevolumenstromes unterstellt. Für die Ermittlung der theoretisch möglichen Wärmepumpenlaufzeit (WLZ) wurde die Anzahl der Messwerte mit einer abgegebenen thermischen Energie $>1 \mathrm{kWh}$ (Kollektornutzenergie) für die jeweilige Jahreszeit mit dem Messintervall (z) multipliziert. Die Berechnung der übrigen Kennwerte erfolgte durch den Wärmetauscherhersteller [74]. Anhand der Soletemperatur ist es jetzt möglich, die Verdampfungstemperatur des Kältemittels zu bestimmen. Laut Herstellerangaben liegt die Verdampfungstemperatur bei den durchschnittlich errechneten Soletemperaturen von $10^{\circ} \mathrm{C}$ bei $0{ }^{\circ} \mathrm{C}$ und für die Soletemperaturen $12{ }^{\circ} \mathrm{C}$ und $14{ }^{\circ} \mathrm{C}$ zwischen $0{ }^{\circ} \mathrm{C}$ und 
$5{ }^{\circ} \mathrm{C}$ [65]. Mit Hilfe des technischen Datenblattes des Verdichters ist es jetzt möglich, die Verdampferleistung (VL) und die elektrische Antriebsleistung (EAL) für die verschiedenen abgegebenen Temperaturniveaus $\mathrm{zu}$ bestimmen [66]. Für die Verdampferleistung und die elektrische Antriebsleistung wurde ein linearer Zusammenhang mit der Verdampfungstemperatur des Kältemittels unterstellt und die Leistungen für die Soletemperatur von $12{ }^{\circ} \mathrm{C}$ und $14{ }^{\circ} \mathrm{C}$ interpoliert. Für eine erste Einschätzung wurde in den folgenden Berechnungen lediglich ein Verdichter betrachtet. Die Leistungszahl ist definiert als das Verhältnis zwischen der abgegebenen Nutzleistung $\left(\dot{\mathrm{Q}}_{0}+\mathrm{P}_{\mathrm{el}}\right)$ und der aufgenommenen elektrischen Antriebsleistung des Verdichters $\left(\mathrm{P}_{\mathrm{el}}\right)$ [63]. Bei einer Wärmepumpe mit Heißgasabschöpfung ist das Temperaturniveau im kältesten Pufferspeicher für die Berechnung der Leistungszahl auschlaggebend [65]. Für sauggasgekühlte Verdichter lässt sich nach Formel (14) die Leistungszahl berechnen [63].

$\varepsilon_{\mathrm{V}}=\frac{\dot{\mathrm{Q}}_{0}+\mathrm{a}_{\mathrm{f}} \cdot \mathrm{P}_{\mathrm{el}}}{\mathrm{P}_{\mathrm{el}}}$

Darin sind:

$\varepsilon_{\mathrm{V}}=$ Leistungszahl

$[-]$

$\dot{\mathrm{Q}}_{0}=$ Verdampferleistung

$[\mathrm{W}]$

$\mathrm{a}_{\mathrm{f}}=$ Anlagenfaktor

$[-]$

$\mathrm{P}_{\mathrm{el}} \quad=$ elektrische Antriebsleistung

[W]

Bei der Berechnung ist der Anlagenfaktor $\left(a_{f}\right)$ laut Wärmepumpenhersteller mit 0,99 anzusetzen und wurde deshalb bei der Berechnung vernachlässigt [65]. Demnach ergeben die Verdampferleistung und die elektrische Antriebsleistung des Verdichters die Wärmeleistung (WL) der Wärmepumpe [45]. 
Die folgende Berechnung der Jahresarbeitszahl sieht nach der Richtlinie VDI 4650 Korrekturfaktoren vor [71]. Da die Grundlage dieser Berechnung die ermittelte Leistungszahl der Wärmepumpe nach DIN EN 14511 und DIN EN 255-3 ist und die Korrekturfaktoren sich auf diese Messungen beziehen, konnten diese nicht berücksichtigt werden. Ebenfalls unberücksichtigt blieb die Trennung der Arbeitszahlen für die Raumheizung und Wasserbereitung, da die Wärmepumpe im Jahresverlauf mit derselben Vorlauftemperatur betrieben werden soll. Entsprechend der Richtlinie wurden Wärmeverluste der Pufferspeicher nicht berücksichtigt. Für die vereinfachte Ermittlung der Jahresarbeitszahl wurden zunächst getrennte Arbeitszahlen für die einzelnen Jahreszeiten berechnet. Zu der elektrischen Antriebsleistung des Verdichters wurden für die Umwälzpumpen die vom Hersteller angegebenen Leistungsaufnahmen herangezogen. Die drei Heizungsumwälzpumpen sind auf Stufe zwei eingestellt und wurden mit je $75 \mathrm{~W}$ berücksichtigt. Die temperaturgesteuerte Solepumpe wurde mit der maximalen Leistungsaufnahme von $258 \mathrm{~W}$ hinzugerechnet. Für die Ventilatorantriebsleistung wurde die mittlere Leistungsaufnahme im Betrachtungszeitraum angerechnet. Die Berechnung der abgegebenen Wärmemenge und aufgenommenen elektrischen Antriebsenergie erfolgte durch Multiplikation mit der theoretisch möglichen Wärmepumpenlaufzeit im jeweiligen Betrachtungszeitraum. Bei dieser Betrachtung wurde eine vollständige Wärmeabnahme durch die Jugendherberge unterstellt. Im Anschluss wurden nach der Formel (15) die Arbeitszahlen für die jeweilige Jahreszeit berechnet [47]. 
$\beta=\frac{\mathrm{W}_{\text {Nutz }}}{\mathrm{W}_{\mathrm{el}}}$

Darin sind:

$\beta=$ Arbeitszahl $[-]$

$\mathrm{W}_{\text {Nutz }}=$ abgegebene Nutzwärme $\quad[\mathrm{kWh}]$

$\mathrm{W}_{\mathrm{el}} \quad=$ gesamte elektrische Antriebsenergie $[\mathrm{kWh}]$

Die Ermittlung der Jahresarbeitszahl erfolgte durch Addieren der abgegebenen Wärmemengen und aufgenommenen Antriebsenergien in den unterschiedlichen Jahreszeiten. Die Jahresarbeitszahl wurde im Anschluss nach Formel (15) berechnet.

Laut der Trinkwasserverordnung darf die angenommene Warmwassertemperatur von $40{ }^{\circ} \mathrm{C}$ erst an der Mischbatterie der Entnahmestelle abgemischt werden $[19,20]$. Aus diesem Grund muss für die Berechnung der Wärmeabnahme der Jugendherberge die abgenommene $60^{\circ} \mathrm{C}$ warme Wassermenge bestimmt werden. Für die Berechnung des Mischverhältnisses bei $40^{\circ} \mathrm{C}\left(\mathrm{T}_{\text {Misch }}\right)$ wurde die Temperatur des warmen Wassers $\left(\mathrm{T}_{\text {warm }}\right)$ mit $60^{\circ} \mathrm{C}$ und die des kalten Wassers $\left(\mathrm{T}_{\text {kalt }}\right)$ mit $10^{\circ} \mathrm{C}$ angenommen. Der Warmwasserverbrauch von 31 I pro Person und Tag wurde für die Masse des warmen Wassers eingesetzt. Die anteilige Kaltwassermenge wurde durch Umstellen der Formel (16) nach $\mathrm{m}_{\text {kalt }}$ ermittelt [40]. 


$$
\mathrm{T}_{\text {Misch }}=\frac{\mathrm{m}_{\text {warm }} \cdot \mathrm{T}_{\text {warm }}+\mathrm{m}_{\text {kalt }} \cdot \mathrm{T}_{\text {kalt }}}{\mathrm{m}_{\text {kalt }}+\mathrm{m}_{\text {warm }}}
$$

Daraus ergibt sich:

$\mathrm{m}_{\text {kalt }}=\frac{\mathrm{m}_{\text {warm }} \cdot \mathrm{T}_{\text {warm }}-\mathrm{T}_{\text {Misch }} \cdot \mathrm{m}_{\text {warm }}}{\mathrm{T}_{\text {Misch }}-\mathrm{T}_{\text {kalt }}}$

Darin sind:

$\mathrm{T}=$ Temperatur $[\mathrm{K}]$

$\mathrm{m}=$ Masse $\quad[\mathrm{kg}]$

Anhand der berechneten Wassermengen wurde das Mischverhältnis bestimmt. Die jeweiligen Wassermengen wurden für einen Warmwasserverbrauch von 31 I pro Person ermittelt. Im Anschluss wurden die Mengenanteile mit den Übernachtungen in der Jugendherberge für den jeweiligen Betrachtungszeitraum multipliziert. Mit dieser Berechnung konnte die Wassermenge bestimmt werden, die durch die Pufferspeicher zur Brauchwasservorerwärmung fließt. Den folgenden Berechnungen liegt aufgrund einer möglichst hohen Arbeitszahl eine Temperatur im kältesten Pufferspeicher (kPS) von $30^{\circ} \mathrm{C}$ zugrunde. Es wurde unterstellt, dass das Brauchwasser nach dem Durchströmen der beiden Pufferspeicher eine Brauchwassertemperatur von $46{ }^{\circ} \mathrm{C}$ erreicht [65]. Diese Annahme beruht auf einem Erfahrungswert des Wärmepumpenherstellers, da der gegenwärtige Zustand der Anlage noch keine Messung der Brauchwassertemperatur zulässt. Mit der berechneten Warmwassermenge und den getroffenen Annahmen wurde nach Formel (13) die theoretisch abgenommene Wärmemenge für die Brauchwasservorerwärmung berechnet. Laut Wärmepumpenhersteller ist erfahrungsgemäß bei einer Wärmepumpe mit drei Heizkreisläufen eine Wärmemengenverteilung von zwei Dritteln für die Brauchwassererwärmung und einem Drittel für die Heizungsunterstützung zu erwarten [65]. Die Gesamtwärmeabnahme (GWA) der Jugendherberge ergibt sich demnach aus der abgenommenen Wärmemenge für die Brauchwasservorerwärmung und 
der mit der halben Wärmemenge der Brauchwasservorerwärmung anzurechnenden Heizungsunterstützung. Es wurde unterstellt, dass die Wärmemenge zur Heizungsunterstützung vollständig von dem konventionellen Heizungssystem abgenommen wird. Für die Ermittlung der Wärmepumpenlaufzeiten wurde die berechnete abgegebene Wärmemenge für eine vollständige Wärmeabnahme dem Abnahmeverhalten der Jugendherberge gegenübergestellt. Durch Subtrahieren der Gesamtwärmeabnahme von der möglichen abgegebenen Wärmemenge wurde die Wärmebedarfsdeckung (WBD) ermittelt. Bei einer negativen Wärmebedarfsdeckung wurde die komplette Wärmepumpenlaufzeit für den Betrachtungszeitraum angerechnet. Bei einem Angebot, das über der Wärmebedarfsdeckung lag, wurde die Gesamtwärmeabnahme der Jugendherberge durch die Wärmeleistung der Wärmepumpe im Betrachtungszeitraum geteilt. Anhand der berechneten Wärmepumpenlaufzeiten wurden analog für den Betrieb bei einer vollständigen Wärmeabnahme die Arbeitszahlen und die Jahresarbeitszahl bei einer Temperatur von $30^{\circ} \mathrm{C}$ im kältesten Pufferspeicher berechnet.

Um die Vorteile einer möglichen technischen Optimierung der Stauwärmenutzungsanlage beurteilen zu können, wurden zusätzliche Berechnungen durchgeführt. Bei der Berechnung des optimierten Wärmepumpenbetriebes wurden eine Direktverdampfung des Kältemittels und eine vollständige Wärmeabnahme durch die Jugendherberge unterstellt. Wird der Kältekreislauf der Wärmepumpe direkt zum Wärmetauscher geleitet, kann aufgrund der herrschenden Druckdifferenz hinter dem Expansionsventil auf eine Pumpe für die Wärmequelle verzichtet werden. Es wurde davon ausgegangen, dass neben der eingesparten Pumpenleistung das Kältemittel bei der Temperatur verdampft, die zuvor für die Sole errechnet wurde. Weiterhin wurde unterstellt, dass die Ventilatorantriebsleistung durch eine Optimierung der Luftabsaugung um $15 \%$ gesenkt werden kann. Die Berechnung der Leistungszahlen, Arbeitszahlen und Jahresarbeitszahlen erfolgte entsprechend den Berechnungen eines Wärmepumpenbetriebes bei einer vollständigen Wärmeabnahme. 
Für die ökonomische Betrachtung des Systems wurden zunächst die Energiebezugskosten ermittelt. Anhand der aufgenommenen elektrischen Antriebsenergie und des Strompreises sind die Energiebezugskosten für den Wärmepumpenbetrieb (SKWP) errechnet worden. Der kalkulierte Strompreis von 13,98 ct/kWh entspricht dem Mittelwert des Haupt- und Nebentarifes der Jugendherberge [64]. Bei der Berechnung der Energiebezugskosten des konventionellen Heizungssystems ist die abgegebene Wärmemenge der Wärmepumpe zugrunde gelegt worden. Zunächst wurde über den NormNutzungsgrad des Kessels und den Gas-Brennwert der benötigte Gasmengenbedarf (GMB) bestimmt. Der Norm-Nutzungsgrad beträgt bei einer Heiztemperatur von $75 / 60{ }^{\circ} \mathrm{C} 95 \%$ und wurde aus dem technischen Datenblatt des Gas-Brennwertkessels entnommen [14, 75]. Für den Brennwert des Gases wurde mit 11,4 kWh/m³ der mittlere Gasbrennwert des örtlichen Gasversorgers angenommen [64]. Die Bemessungsgrundlage für die Gasabrechnung (BGGA) errechnet sich aus der verbrauchten Gasmenge multipliziert mit dem Brennwert des Gases. Durch die Multiplikation mit dem Gaspreis pro Kilowattstunde ergeben sich die Gaskosten (GK) der Jugendherberge. Der Gaspreis der Jugendherberge beträgt 5,12 ct/kWh [64]. Durch das Subtrahieren der Stromkosten für den Wärmepumpenbetrieb von den Gaskosten des konventionellen Heizungssystems wurden die eingesparten Energiebezugskosten (eEBK) für den jeweiligen Betrachtungszeitraum ermittelt.

Für die Berechnung des Amortisationszeitraumes (AZR) wurden die Investitionskosten (IK) der Stauwärmenutzungsanlage ermittelt. Auf die Berücksichtigung weiterer Kostenansätze wurde verzichtet, da es sich bei dieser Anlage um eine Pilotanlage zur Praxiserprobung handelt. Es wurde unterstellt, dass die Investitionskosten für alle drei Betriebsmodelle gleich sind. Der Amortisationszeitraum wurde durch Dividieren der eingesparten Energiebezugskosten durch die Investitionskosten ermittelt. Diese ökonomische Betrachtung wurde für alle drei Betriebsmodelle bei einer Temperatur von $30^{\circ} \mathrm{C}$ im kältesten Pufferspeicher durchgeführt. Alle Ergebnisse wurden nach der Berechnung auf zwei Stellen hinter dem Komma gerundet. 


\section{$4 \quad$ Ergebnisse}

Bei der Ergebnisauswertung wird zunächst nur der Solardach-Luft-Kollektor ohne das dahinter stehende Nutzungssystem betrachtet. Für die Darstellung der Ergebnisse wurden repräsentative Tage ausgewählt. Im zweiten Schritt werden bei den Berechnungen sämtliche aufgezeichneten Messwerte des Solardach-Luft-Kollektors mit dem dahinter stehenden Nutzungssystem in Beziehung gebracht.

\subsection{Solardach-Luft-Kollektor}

\subsubsection{Globalstrahlungsverlauf in Kollektorebene}

In Abbildung 13 ist die Globalstrahlung in der Kollektorebene in Abhängigkeit von der Zeit für ausgewählte sonnige Tage im Jahresverlauf dargestellt. Die abgebildeten Messwerte zeigen einen Glockenkurvenverlauf der Globalstrahlung im Tagesgang. Der Vergleich der Globalstrahlungsverläufe verdeutlicht die unterschiedliche Sonnenscheindauer in den einzelnen Jahreszeitabschnitten. Auffällig ist, dass der Verlauf der Globalstrahlung an allen Tagen in der ersten Tageshälfte steiler verläuft als in der zweiten Tageshälfte. Dagegen ist bei Sonnenaufgang und Sonnenuntergang im Vergleich zum restlichen Tagesgang ein deutlich flacherer Globalstrahlungsverlauf zu beobachten. Diese Beobachtung ist bei Sonnenuntergang deutlich ausgeprägter als bei Sonnenaufgang. Die dargestellten Globalstrahlungswerte haben für den Sommer- und Frühlingstag eine identische Höhe. Besonders auffallend sind die Ausreißer über der Globalstrahlungskurve des sonnigen Sommertages in der ersten Tageshälfte. Der Herbsttag weist im Vergleich zum Frühlingstag deutlich niedrigere Globalstrahlungswerte auf. Die niedrigsten Globalstrahlungswerte besitzt der sonnige Wintertag. 


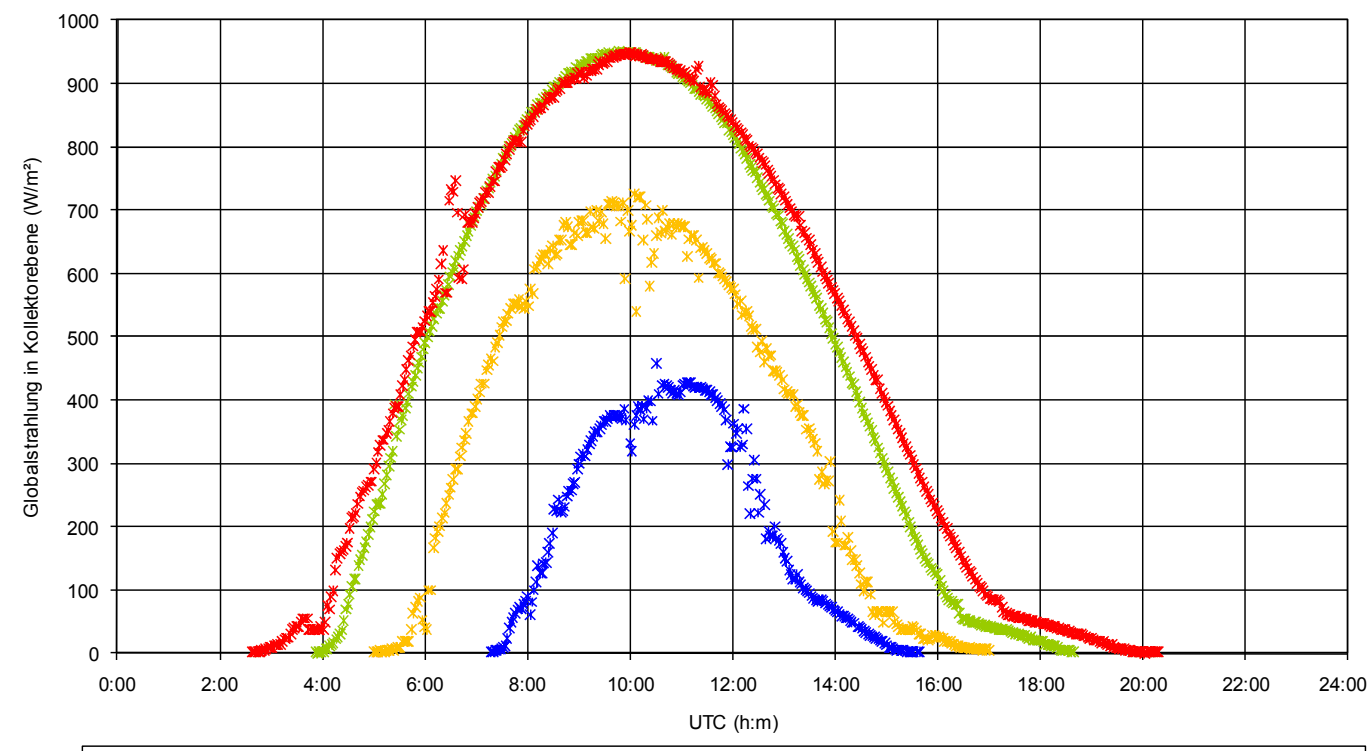

* sonniger Wintertag (22.01.2009) * sonniger Frühlingstag (21.04.2009) * sonniger Sommertag (02.07.2009) $*$ sonniger Herbsttag (06.10.2009)

Abbildung 13: Globalstrahlungsverläufe in der Kollektorebene für ausgewählte sonnige Tage im Jahresverlauf in Abhängigkeit von der Zeit

\subsubsection{Temperaturerhöhung}

Die Temperaturerhöhung der Kollektoreintrittsluft ist in Abbildung 14 als Temperaturdifferenz $\left(T_{a}-T_{e}\right)$ zwischen der Kollektoraustrittsluft $\left(T_{a}\right)$ und Kollekoreintrittsluft $\left(T_{e}\right)$ in Abhängigkeit von der Zeit für ausgewählte sonnige Tage im Jahresverlauf dargestellt. Der Vergleich von Abbildung 13 und Abbildung 14 zeigt einen ähnlichen Verlauf der Temperaturerhöhung und des Globalstrahlungsverlaufes. Die Beobachtung des flacheren Verlaufes der Globalstrahlung bei Sonnenaufgang und Sonnenuntergang spiegelt sich bei allen Temperaturdifferenzverläufen wider. Die Ausreißer über der Globalstrahlungskurve des sonnigen Sommertages in der ersten Tageshälfte sind auch im Verlauf der Temperaturdifferenz wiederzufinden. Auffällig ist der Verlauf der Temperaturdifferenz des sonnigen Frühlings- und Sommertages. Trotz der zuvor in Abbildung 13 beschriebenen gleichen Globalstrahlungsintensität ist die Temperaturdifferenz des sonnigen Sommertages am Nachmittag deutlich höher als die des sonnigen Frühlingstages. Es ist weiterhin auffällig, dass bei diesen beiden Tagen im Bereich der maximalen Messwerte im Gegensatz zum Globalstrahlungsverlauf Unregelmäßigkeiten 
auftreten. Besonders auffällig ist, dass bei allen dargestellten Tagen in den Nachmittagsstunden ein höheres Temperaturniveau als in den Vormittagsstunden erreicht wird. Besonders deutlich wird dies dadurch, dass bei Sonnenuntergang eine deutlich höhere Temperaturdifferenz als bei Sonnenaufgang abzulesen ist. Die mittlere Umgebungstemperatur zwischen Sonnenaufgang und Sonnenuntergang betrug für den Wintertag $2{ }^{\circ} \mathrm{C}$ und für den Frühlingstag $10^{\circ} \mathrm{C}$. Für den Sommertag belief sich die mittlere Umgebungstemperatur auf $22^{\circ} \mathrm{C}$ und für den Herbsttag auf $14{ }^{\circ} \mathrm{C}$. Die mittlere Windgeschwindigkeit für den Wintertag lag bei $6,4 \mathrm{~m} / \mathrm{s}$ bei einer Schwankungsbreite zwischen $2 \mathrm{~m} / \mathrm{s}$ und $10 \mathrm{~m} / \mathrm{s}$. Die Schwankungsbreite für den Frühlingstag lag zwischen $1 \mathrm{~m} / \mathrm{s}$ und $6 \mathrm{~m} / \mathrm{s}$ bei einer mittleren Windgeschwindigkeit von $3,6 \mathrm{~m} / \mathrm{s}$. Für den Sommertag wurde eine mittlere Windgeschwindigkeit von $3,0 \mathrm{~m} / \mathrm{s}$ mit einer Schwankungsbreite zwischen $1 \mathrm{~m} / \mathrm{s}$ und $5 \mathrm{~m} / \mathrm{s}$ gemessen. Der Herbsttag hatte eine mittlere Windgeschwindigkeit von $4,9 \mathrm{~m} / \mathrm{s}$ mit einer Schwankungsbreite zwischen $1 \mathrm{~m} / \mathrm{s}$ und $10 \mathrm{~m} / \mathrm{s}$. Aus Übersichtlichkeitsgründen sind in Abbildung 14 und den folgenden Abbildungen die möglichen negativen und positiven Temperaturdifferenzen an klaren und bewölkten Nächten nicht dargestellt. 


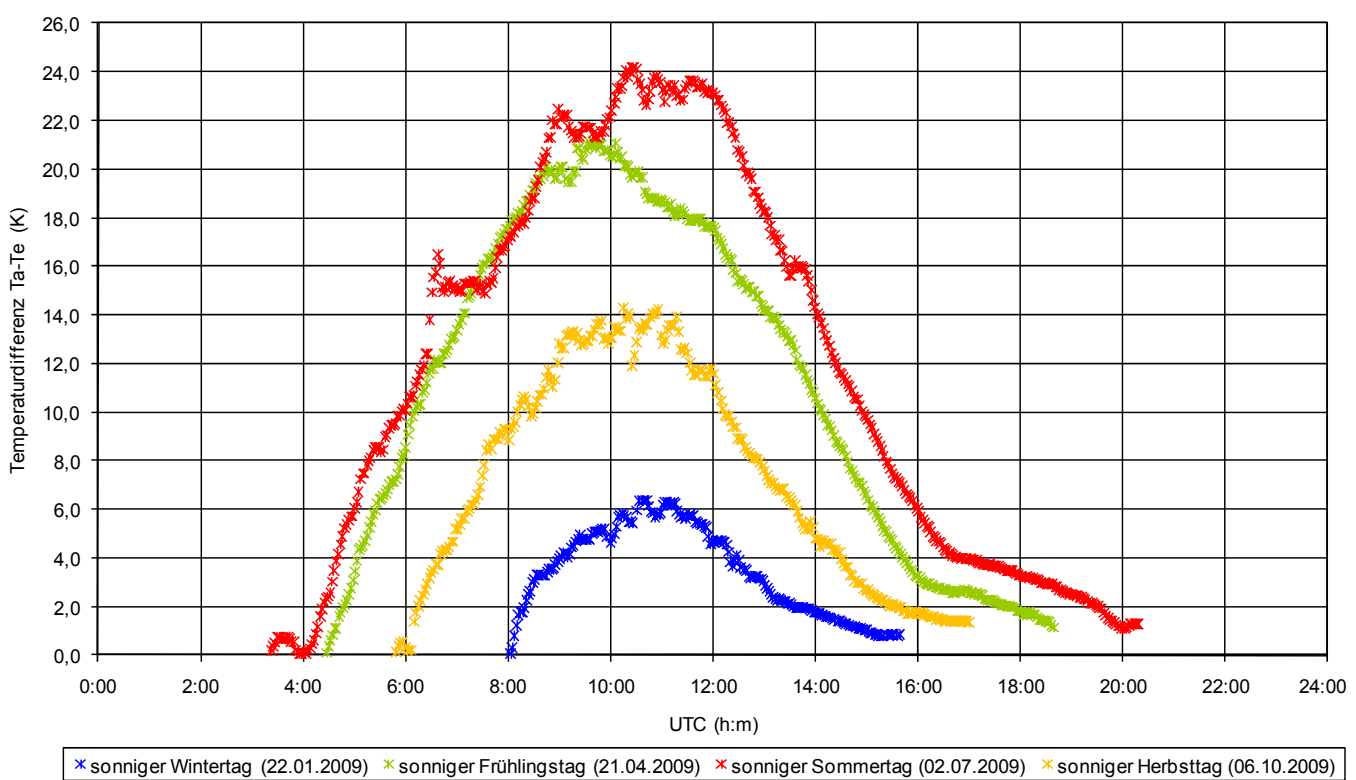

Abbildung 14: Verlauf der Temperaturdifferenzen des Solardach-Luft-Kollektors für ausgewählte sonnige Tage im Jahresverlauf von Sonnenaufgang bis Sonnenuntergang in Abhängigkeit von der Zeit $T_{a}$, Austrittstemperatur; $T_{e}$, Eintrittstemperatur.

Der Verlauf der Temperaturerhöhung der Kollektoreintrittsluft ist in Abbildung 15 exemplarisch für einen sonnigen Frühlingstag dargestellt. Die Abbildung zeigt die Temperaturdifferenz $\left(T_{a}-T_{e}\right)$ zwischen der Kollektoraustrittsluft $\left(T_{a}\right)$ und Kollektoreintrittsluft $\left(\mathrm{T}_{\mathrm{e}}\right)$ in Abhängigkeit von der Globalstrahlung. Aus der Abbildung ist zu entnehmen, dass in der ersten Tageshälfte mit einer Globalstrahlungsintensität unterhalb von $70 \mathrm{~W} / \mathrm{m}^{2}$ keine positive Temperaturdifferenz erzielt wird. Der Vergleich mit Abbildung 14 bestätigt die höhere Temperaturdifferenz in der zweiten Tageshälfte zwischen der Kollektoraustrittsluft und Kollektoreintrittsluft. Die Temperaturdifferenz in der zweiten Tageshälfte liegt zunächst unterhalb der ersten Tageshälfte. Ab einer Globalstrahlungsintensität von $850 \mathrm{~W} / \mathrm{m}^{2}$ nimmt die Temperaturdifferenz der zweiten Tageshälfte mit Abnahme der Globalstrahlung tendenziell gegenüber der ersten Tageshälfte zu. In Abbildung 16 werden aus diesem Grund die Tage zur Darstellung in eine erste und zweite Tageshälfte aufgeteilt. 


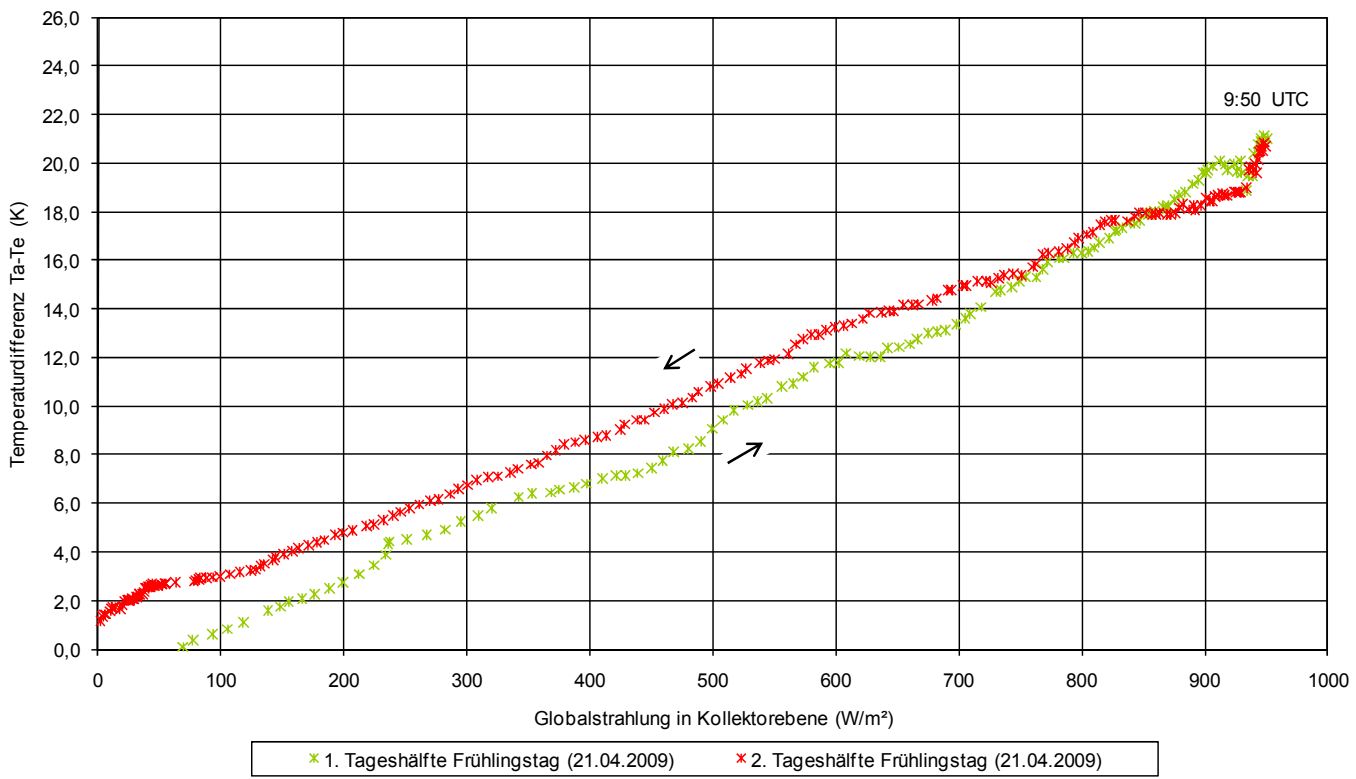

Abbildung 15: Verlauf der Temperaturdifferenz des Solardach-Luft-Kollektors für einen ausgewählten sonnigen Frühlingstag (21.04.2009) in Abhängigkeit von der Globalstrahlung in Kollektorebene $T_{a}$, Austrittstemperatur; $T_{e}$, Eintrittstemperatur.

Der Zusammenhang zwischen der Temperaturerhöhung der Kollektoreintrittsluft wird in Abbildung 16 in Abhängigkeit von der Globalstrahlung verdeutlicht. Die Temperaturdifferenzen $\left(T_{a}-T_{e}\right)$ für ausgewählte sonnige Tage im Jahresverlauf wurden in Form von linearen Regressionsgeraden für die erste und zweite Tageshälfte grafisch dargestellt. Das hohe Bestimmtheitsmaß der Regressionsgeraden bestätigt den linearen Zusammenhang zwischen der Temperaturdifferenz und der Globalstrahlungsintensität. Die Steigung der Geraden zeigt, dass mit Zunahme der Globalstrahlungsintensität die Temperaturdifferenz zwischen der Kollektoraustrittsluft und Kollektoreintrittsluft proportional ansteigt. In der ersten Tageshälfte liegen die Temperaturdifferenzen bei Sonnenaufgang relativ dicht zusammen. Positive Temperaturdifferenzen werden bereits bei niedrigen Globalstrahlungsintensitäten erzielt. Auffällig ist, dass sich bis auf den sonnigen Sommertag die Regressionsgeraden mit Zunahme der Globalstrahlungsintensität annähern. Mit Abnahme der Globalstrahlungs- 
intensität in der zweiten Tageshälfte laufen die Regressionsgeraden tendenziell auf dieselbe Temperaturdifferenz zu. Dieses Temperaturniveau liegt bei allen Geraden deutlich höher als in der ersten Tageshälfte.

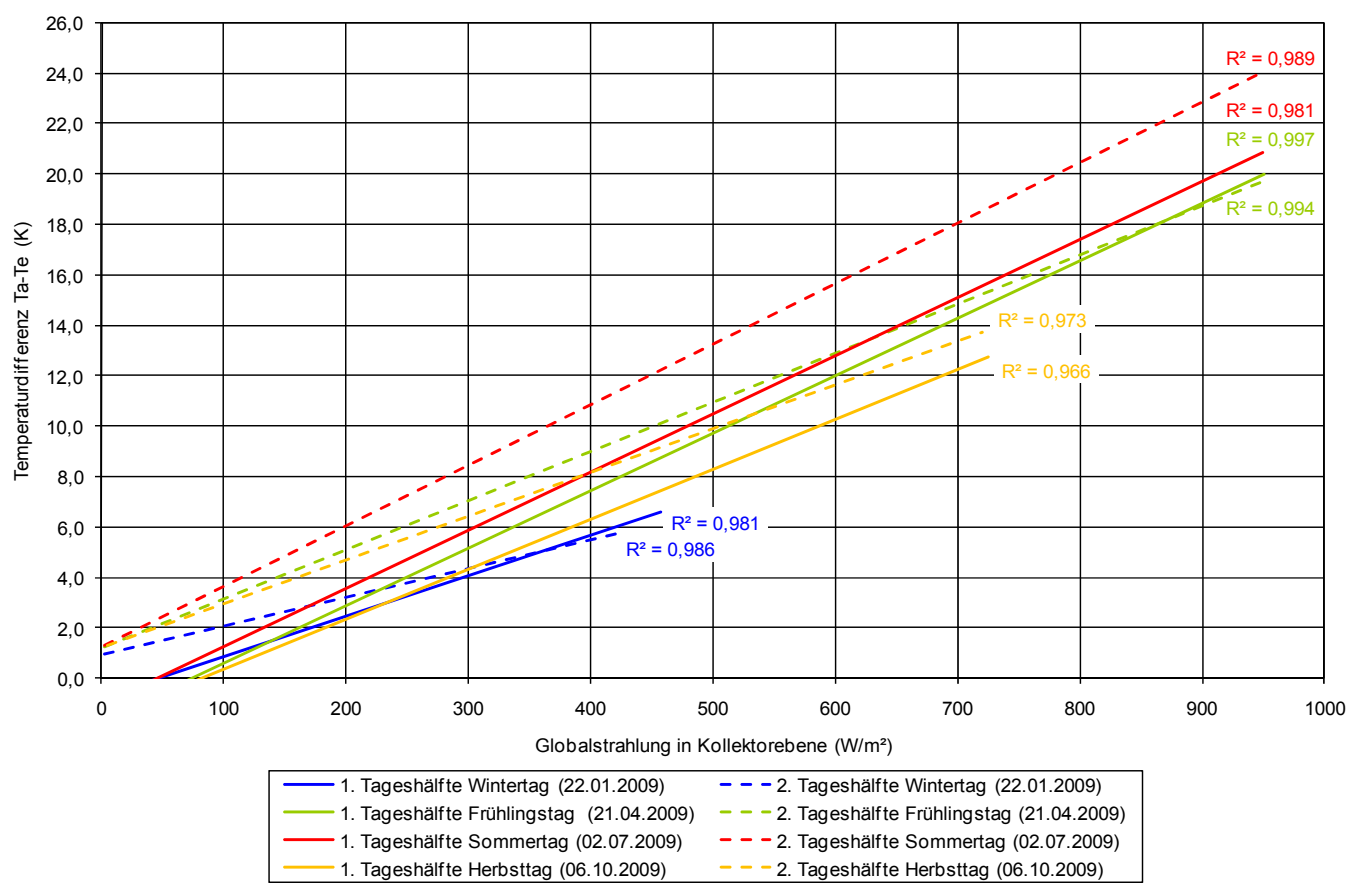

Abbildung 16: Temperaturdifferenzen des Solardach-Luft-Kollektors, dargestellt als lineare Regressionsgeraden, für ausgewählte sonnige Tage im Jahresverlauf in der ersten und zweiten Tageshälfte in Abhängigkeit von der Globalstrahlung in Kollektorebene

$\mathrm{T}_{\mathrm{a}}$, Austrittstemperatur; $\mathrm{T}_{\mathrm{e}}$, Eintrittstemperatur.

\subsubsection{Betriebsverhalten}

Anhand eines wolkigen und eines sonnigen Tages im Frühling wird im Folgenden exemplarisch das Betriebsverhalten des Solardach-Luft-Kollektors beschrieben. In Abbildung 17 sind die Temperaturdifferenzen und der Globalstrahlungsverlauf in Abhängigkeit von der Zeit dargestellt. Die Abbildung zeigt den bereits dargestellten Globalstrahlungsverlauf des sonnigen Frühlingstages (Abb. 13) mit den dazugehörigen Temperaturdifferenzen $\left(T_{a}-T_{e}\right)$ zwischen der Kollektoraustrittsluft $\left(T_{a}\right)$ und Kollektor- 
eintrittsluft $\left(T_{e}\right)$ (vgl. Abb. 14). Zum Vergleich der Temperaturdifferenzen $\left(\mathrm{T}_{\mathrm{a}}-\mathrm{T}_{\mathrm{e}}\right)$ zwischen der ersten und zweiten Tageshälfte wurden die Messwerte der ersten Tageshälfte gespiegelt dargestellt. Diese gespiegelten Werte sind in Abbildung 17 in dem Zeitfenster der zweiten Tageshälfte rot gekennzeichnet. Die gespiegelten Temperaturdifferenzen der ersten Tageshälfte verdeutlichen die höheren Temperaturdifferenzen in der zweiten Tageshälfte. Die Differenzen aus der zweiten Tageshälfte und der gespiegelten ersten Tageshälfte sind in grau dargestellt und bilden den Zeitraum der höheren Temperaturdifferenzen $\left(T_{a}-T_{e}\right)$ in der zweiten Tageshälfte ab. Anhand der Globalstrahlungswerte ist der Beginn der höheren Temperaturdifferenz $\left(\mathrm{T}_{\mathrm{a}}-\mathrm{T}_{\mathrm{e}}\right)$ in der zweiten Tageshälfte mit dem Temperaturdifferenzverlauf in Abbildung 15 zu vergleichen. Der Vergleich der Globalstrahlungswerte zeigt, dass die höheren Temperaturdifferenzen in beiden Abbildungen nicht unmittelbar nach dem maximalen Globalstrahlungswert beginnen. Aus Abbildung 15 ist zu entnehmen, dass bei einer Abnahme der Globalstrahlungsintensität die Spreizung der ersten und zweiten Tageshälfte ab einem Globalstrahlungswert von $850 \mathrm{~W} / \mathrm{m}^{2}$ (11:40 UTC) zunimmt. Der Globalstrahlungswert des Startzeitpunktes der grau dargestellten Kurve aus Abbildung 17 liegt dagegen mit $880 \mathrm{~W} / \mathrm{m}^{2}$ (11:20 UTC) knapp über dem Globalstrahlungswert aus Abbildung 15. Demnach befindet sich der in Abbildung 17 dargestellte Startzeitpunkt 20 min vor dem Beginn der höheren Temperaturdifferenz $\left(T_{a}-T_{e}\right)$ aus Abbildung 15. Die in Abbildung 17 dargestellten Temperaturdifferenzen zwischen den Dachraum- und Umgebungstemperaturen zeigen, dass die Dachraumtemperatur deutlich über der Umgebungstemperatur liegt und mit Zunahme der Globalstrahlungsintensität zeitverzögert ansteigt. Die Verringerung der Temperaturdifferenz mit Abnahme der Strahlungsintensität verläuft deutlich langsamer und erreicht bis Mitternacht nicht ihren ursprünglichen Ausgangswert. Auffällig ist, dass der Verlauf der höheren Temperaturdifferenz $\left(T_{a}-T_{e}\right)$ in der zweiten Tageshälfte dem Anstieg der Differenz zwischen Dachraumund Umgebungstemperatur folgt. 


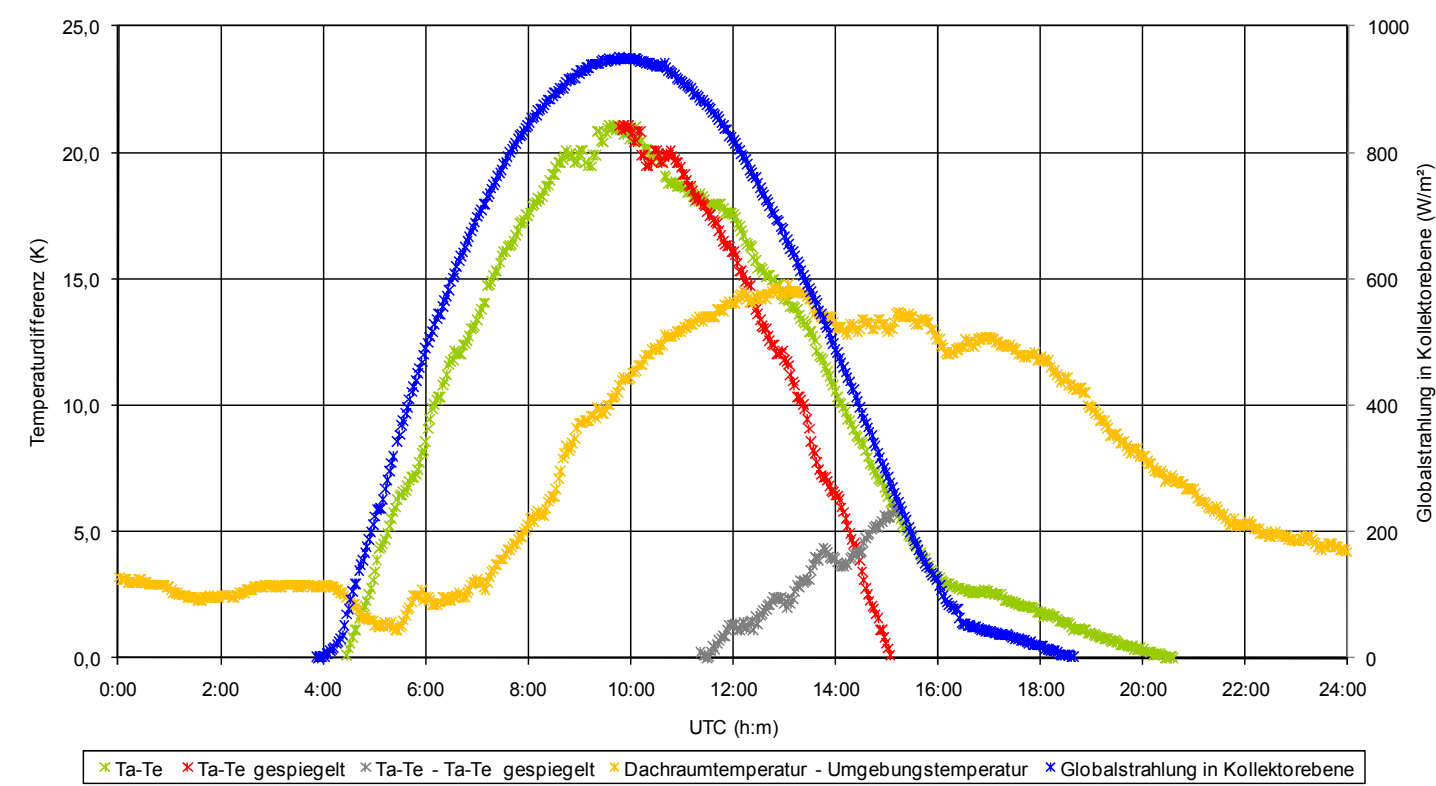

Abbildung 17: Temperaturdifferenzen des Solardach-Luft-Kollektors, der Dachraumund Umgebungstemperatur mit dem Globalstrahlungsverlauf in Kollektorebene und der Spiegelung der Temperaturdifferenz der ersten Tageshälfte an einem sonnigen Frühlingstag (21.04.2009) in Abhängigkeit von der Zeit

$T_{a}$, Austrittstemperatur; $T_{e}$, Eintrittstemperatur.

Die Abbildung 18 zeigt die Temperaturdifferenzen und den Globalstrahlungsverlauf in Abhängigkeit von der Zeit für einen wolkigen Frühlingstag. Die Temperaturdifferenzen und die Globalstrahlungsintensitäten sind im Vergleich zu Abbildung 17 weniger ausgeprägt und deutlich unregelmäßiger im Verlauf. Auffällig ist, dass bereits zwei Stunden nach Sonnenuntergang die Temperaturdifferenzen zwischen den Dachraum- und Umgebungstemperaturen ihre Ausgangswerte vor Sonnenaufgang annähernd erreicht haben. Wie in Abbildung 17 liegt die Dachraumtemperatur auch an einem wolkigen Frühlingstag über der Umgebungstemperatur. 


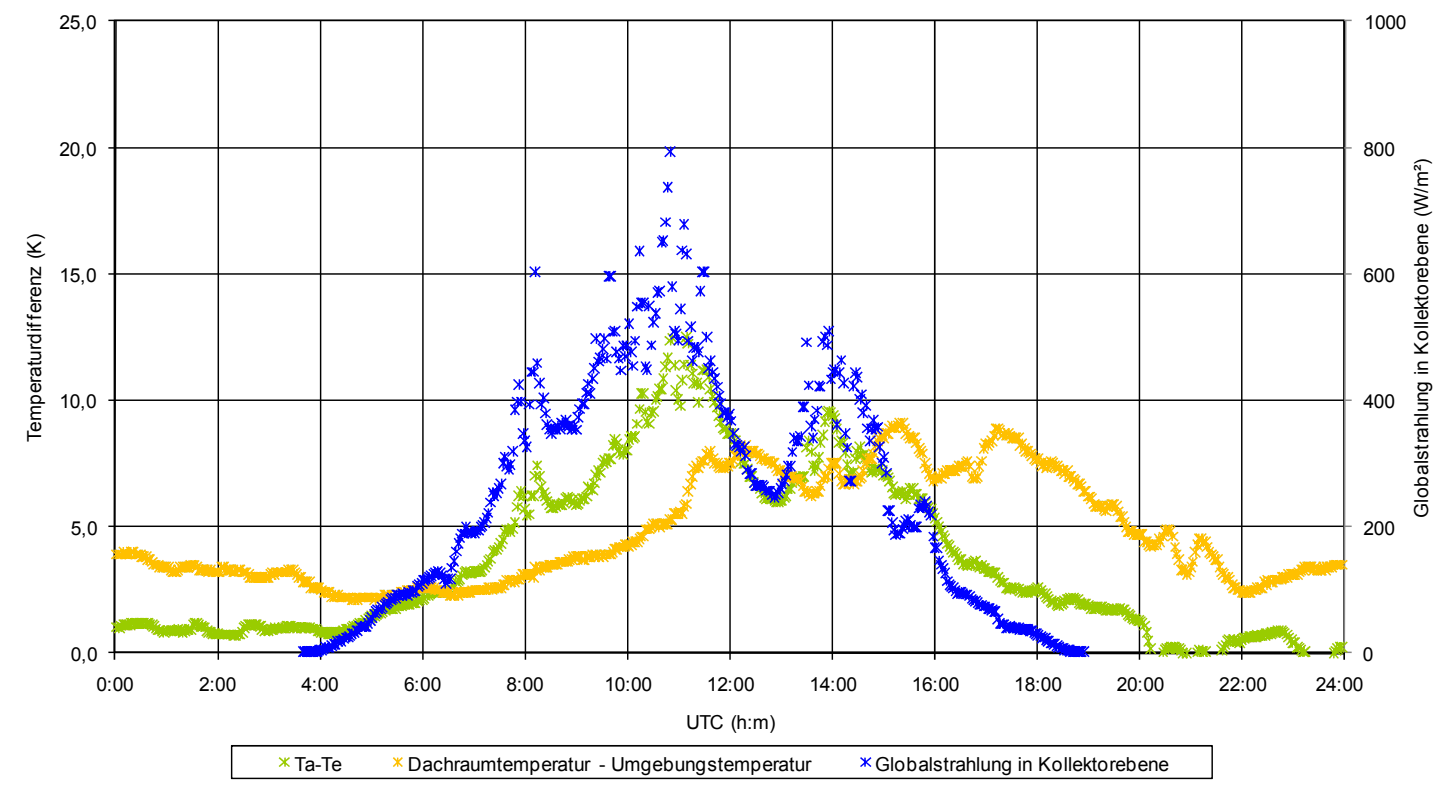

Abbildung 18: Temperaturdifferenzen des Solardach-Luft-Kollektors, der Dachraumund Umgebungstemperatur mit dem Globalstrahlungsverlauf in Kollektorebene an einem wolkigen Frühlingstag (28.04.2009) in Abhängigkeit von der Zeit

$\mathrm{T}_{\mathrm{a}}$, Austrittstemperatur; $\mathrm{T}_{\mathrm{e}}$, Eintrittstemperatur.

Abbildung 19 zeigt den Verlauf der Temperaturdifferenz $\left(\mathrm{T}_{\mathrm{a}}-\mathrm{T}_{\mathrm{e}}\right)$ zwischen der Kollektoraustrittsluft $\left(T_{a}\right)$ und Kollektoreintrittsluft $\left(T_{e}\right)$ sowie den Verlauf der Globalstrahlung in Abhängigkeit von der Zeit für einen wolkigen Frühlingstag. Für die Verlaufsdarstellung wurden die gleitenden Mittelwerte der Periode zwei in die Abbildung 19 eingefügt. Den Verläufen ist zu entnehmen, dass die Temperaturdifferenz annähernd parallel dem Globalstrahlungsverlauf folgt. Der geringe zeitliche Versatz der Maxima zeigt die schnelle Reaktionszeit des Solardach-Luft-Kollektors. 


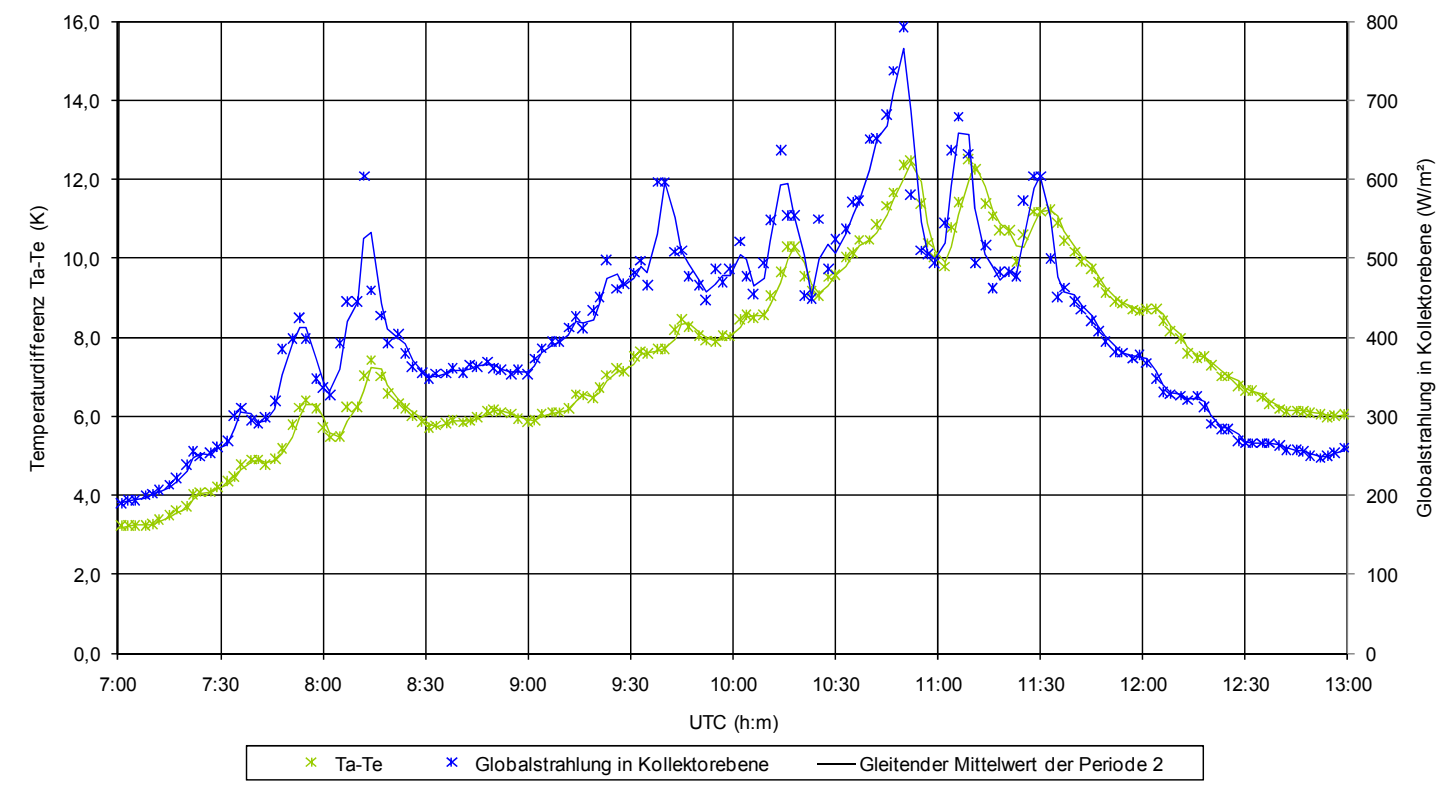

Abbildung 19: Verlauf der Temperaturdifferenz des Solardach-Luft-Kollektors und der Globalstrahlung in Kollektorebene mit gleitenden Mittelwerten der Periode zwei an einem wolkigen Frühlingstag (28.04.2009) in Abhängigkeit von der Zeit

$\mathrm{T}_{\mathrm{a}}$, Austrittstemperatur; $\mathrm{T}_{\mathrm{e}}$, Eintrittstemperatur.

\subsubsection{Kollektorwirkungsgrad}

Der Wirkungsgrad $(\eta)$ des Solardach-Luft-Kollektors ist in Abbildung 20 in Abhängigkeit von der Zeit für ausgewählte sonnige Tage im Jahresverlauf dargestellt. Der Vergleich mit Abbildung 13 zeigt, dass die Wirkungsgrade mit Zunahme der Globalstrahlungsintensität steil ansteigen. Herauszustellen ist, dass die Wirkungsgrade für den Frühlingstag von 6:30 bis 13:00 UTC und für den Wintertag von 8:30 bis 12:00 UTC relativ waagerecht verlaufen. Die Wirkungsgrade des Sommer- und Herbsttages steigen dagegen im Tagesverlauf kontinuierlich an. Im Vergleich mit Abbildung 13 ist besonders auffällig, dass an allen Tagen der Wirkungsgrad mit Abnahme der Strahlungsintensität deutlich zunimmt. 


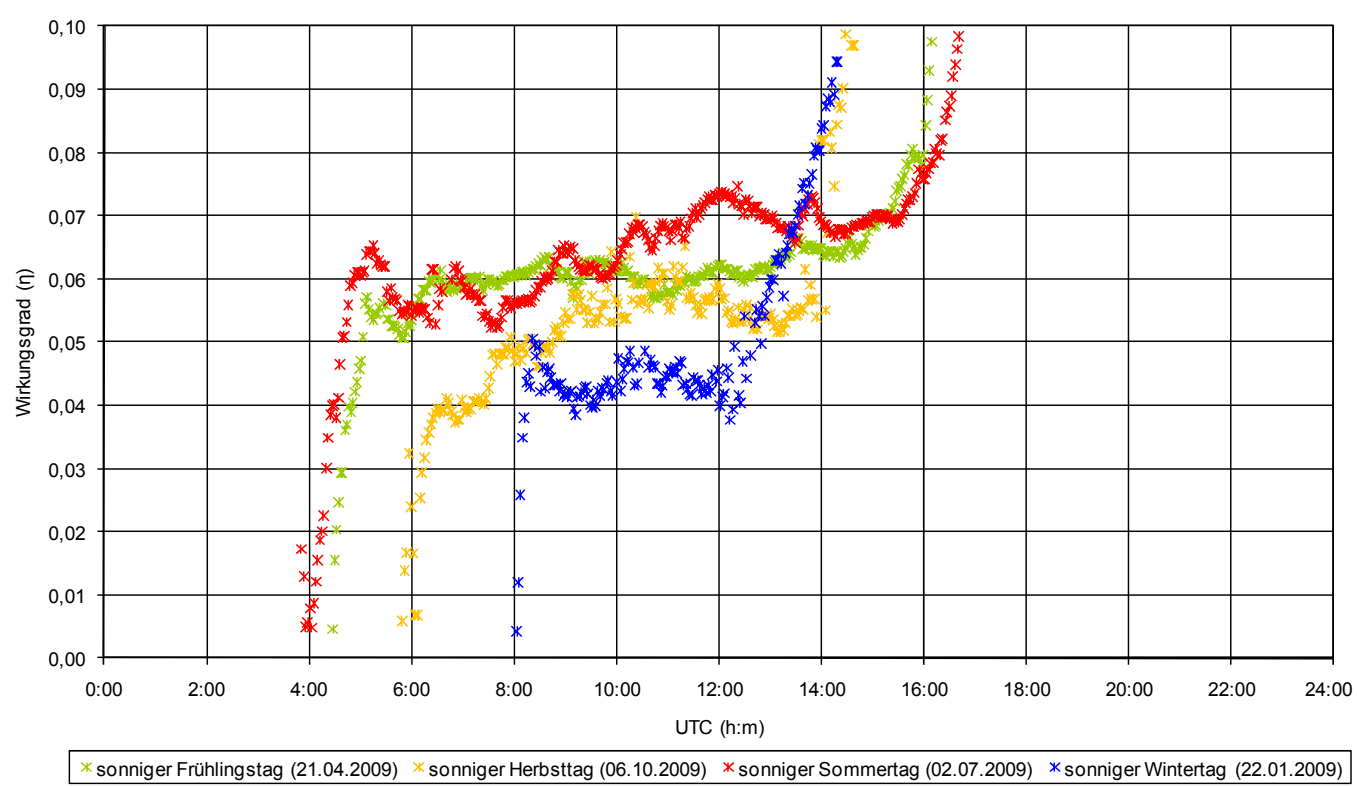

Abbildung 20: Wirkungsgradverläufe des Solardach-Luft-Kollektors für ausgewählte sonnige Tage im Jahresverlauf in Abhängigkeit von der Zeit

In Abbildung 21 ist der Wirkungsgradverlauf $(\eta)$ exemplarisch für einen sonnigen Frühlingstag in Abhängigkeit von der Globalstrahlung dargestellt. Der Wirkungsgradverlauf zeigt ebenfalls wie bei der zuvor dargestellten Temperaturdifferenz $\left(T_{a}-T_{e}\right.$ ) (Abb. 15) eine deutliche Abweichung zwischen den Tageshälften. Die Unterschiede zwischen den einzelnen Tageshälften sind jedoch in Abbildung 21 deutlich ausgeprägter. Der Wirkungsgrad steigt in der ersten Tageshälfte mit zunehmender Globalstrahlungsintensität an. Zwischen den Globalstrahlungswerten von $940 \mathrm{~W} / \mathrm{m}^{2}$ und $850 \mathrm{~W} / \mathrm{m}^{2}$ liegt der Wirkungsgrad der zweiten Tageshälfte unter dem Wirkungsgrad der ersten Tageshälfte. Mit der Abnahme der Globalstrahlungsintensität nimmt der Wirkungsgrad in der zweiten Tageshälfte ab einem Globalstrahlungswert von $850 \mathrm{~W} / \mathrm{m}^{2}$ kontinuierlich $\mathrm{zu}$ und erreicht sein Maximum bei einem Globalstrahlungswert unter $100 \mathrm{~W} / \mathrm{m}^{2}$. Aufgrund der Wirkungsgradzunahme mit abnehmender Globalstrahlungsintensität in der zweiten Tageshälfte ist kein plausibler Zusammenhang zwischen dem Wirkungsgrad und der Globalstrahlung erkennbar. Die Wirkungsgrade der zweiten Tageshälfte sind 
aus diesem Grund nicht bestimmbar und es wird an dieser Stelle auf die Diskussion in Kapitel 5.1.3 verwiesen.

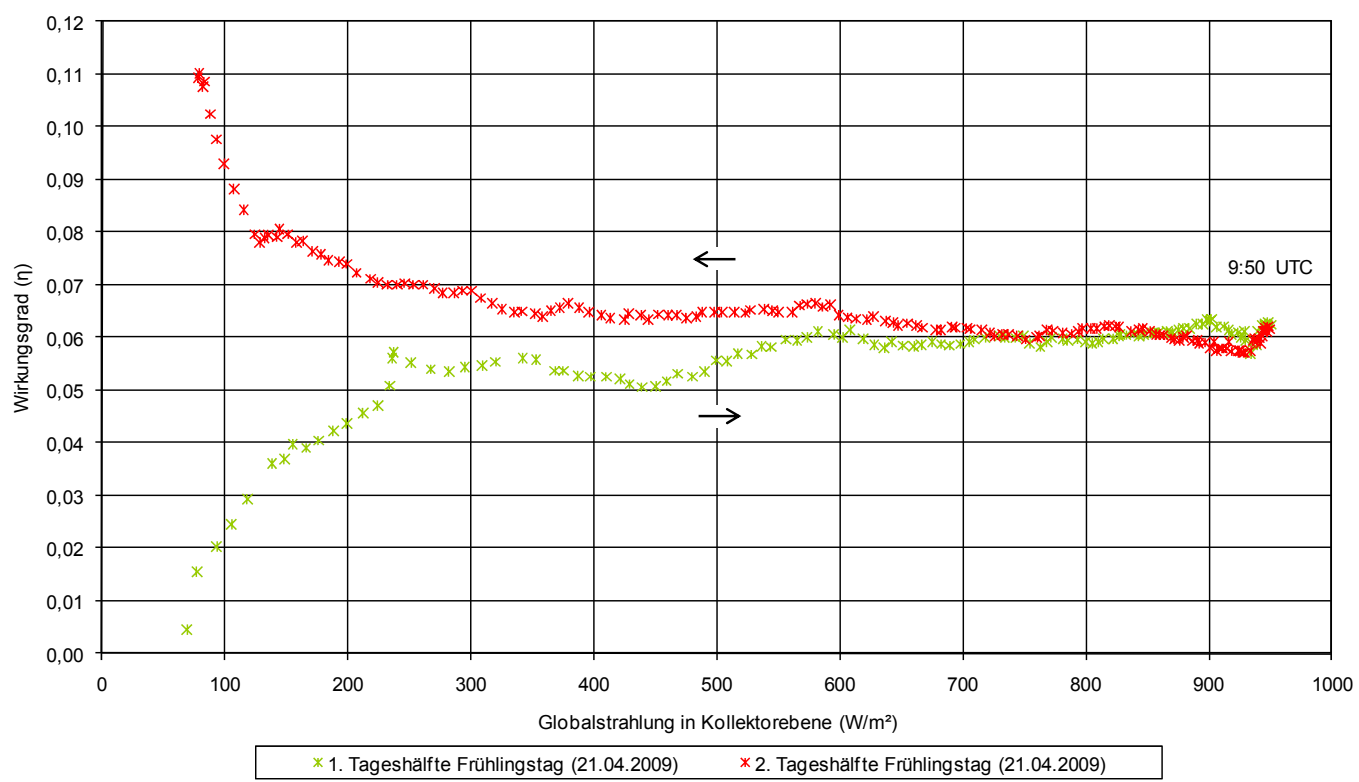

Abbildung 21: Wirkungsgradverlauf des Solardach-Luft-Kollektors für einen ausgewählten sonnigen Tag im Frühling (21.04.2008) in Abhängigkeit von der Globalstrahlung in Kollektorebene

Die Abhängigkeit des Wirkungsgradverlaufes von der Globalstrahlung zeigt Abbildung 22. Die Wirkungsgrade $(\eta)$ für ausgewählte sonnige Tage im Jahresverlauf wurden in Form von logarithmischen Regressionsgeraden für die erste Tageshälfte grafisch abgebildet. Für diese Darstellung wurden die Ausreißer entfernt. Die erzielten Wirkungsgrade in der ersten Tageshälfte bewegen sich zwischen 0,08 und 6,4\%. Mit Zunahme der Strahlungsintensität steigen auch die Wirkungsgrade an. Bei niedrigeren Globalstrahlungsintensitäten steigen die Wirkungsgrade steiler an als bei höheren Globalstrahlungsintensitäten. Für den Frühlings- und Herbsttag konnten höhere Bestimmtheitsmaße als für den Sommer- und Wintertag erzielt werden. Auffällig ist, dass der Wirkungsgradverlauf des Wintertages über dem Wirkungsgradverlauf des Herbsttages liegt. 


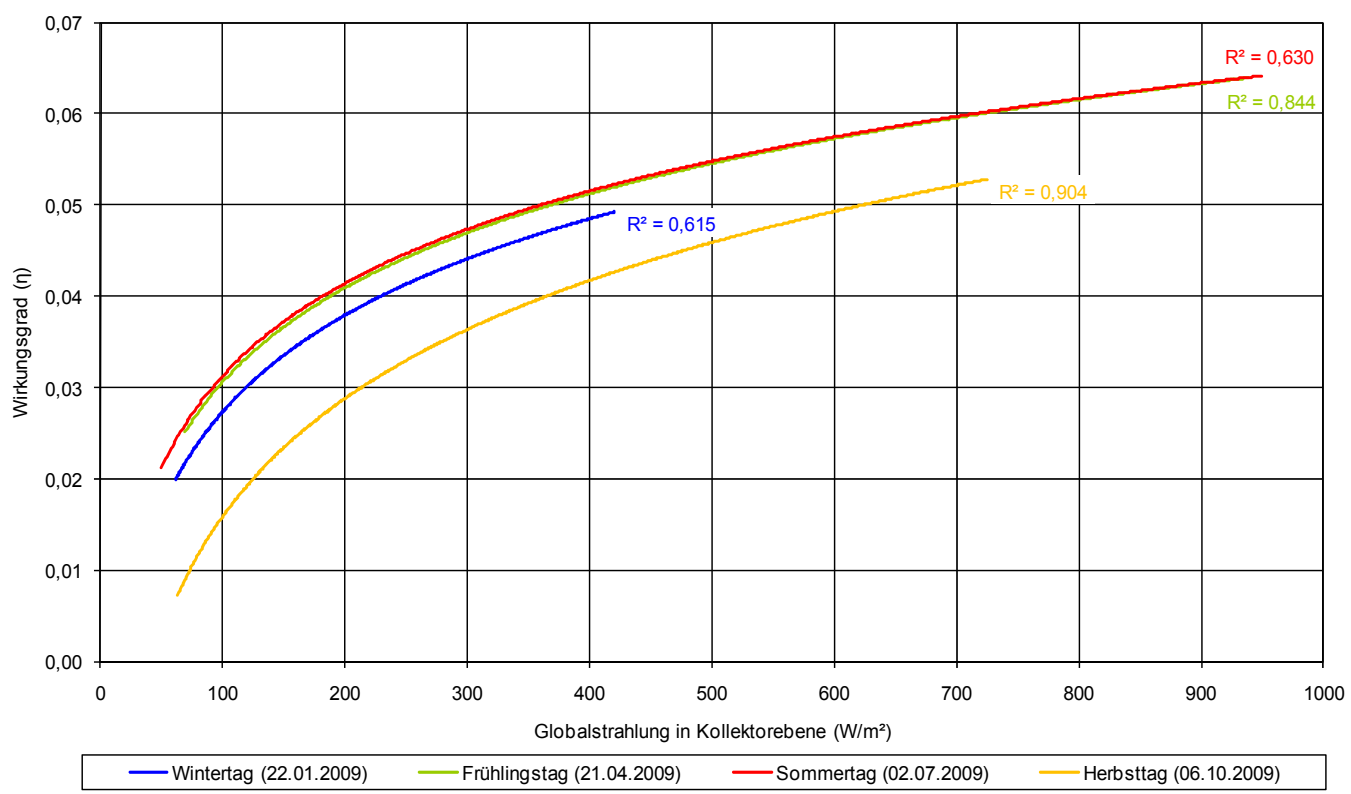

Abbildung 22: Wirkungsgradverläufe des Solardach-Luft-Kollektors, dargestellt als logarithmische Regressionsgeraden, für ausgewählte sonnige Tage im Jahresverlauf in der ersten Tageshälfte in Abhängigkeit von der Globalstrahlung in Kollektorebene

\subsubsection{Einflussfaktoren auf den Luftvolumenstrom}

In der Abbildung 23 werden die Ergebnisse des Versuches zur Bestimmung der Einflussfaktoren auf den geförderten Luftvolumenstrom $\left(\dot{V}_{L}\right)$ in Abhängigkeit von den Kollektorfeldern (KF) dargestellt. Für die Darstellung wurde innerhalb einer Versuchsgruppe der Mittelwert (MW) gebildet. Die Abbildung zeigt, dass der Luftvolumenstrom durch das Schließen einzelner Kollektorfelder abnimmt. Tendenziell ist der Abbildung zu entnehmen, dass innerhalb einer Versuchsgruppe relativ geringe Luftvolumenstromschwankungen auftreten. Der Vergleich mit den dazugehörigen Standardabweichungen (STAW) in Tabelle 1 bestätigt die geringe Schwankungsbreite von maximal $36 \mathrm{~m}^{3} / \mathrm{h}$ um den Mittelwert. Besonders herauszustellen ist, dass mit nur einem geöffneten Kollektorfeld der Mittelwert des geförderten Luftvolumenstromes um $28 \%$ vom Ausgangswert (AW) abweicht, während in den anderen Versuchsgruppen die Abweichungen unter 9,5\% liegen. 


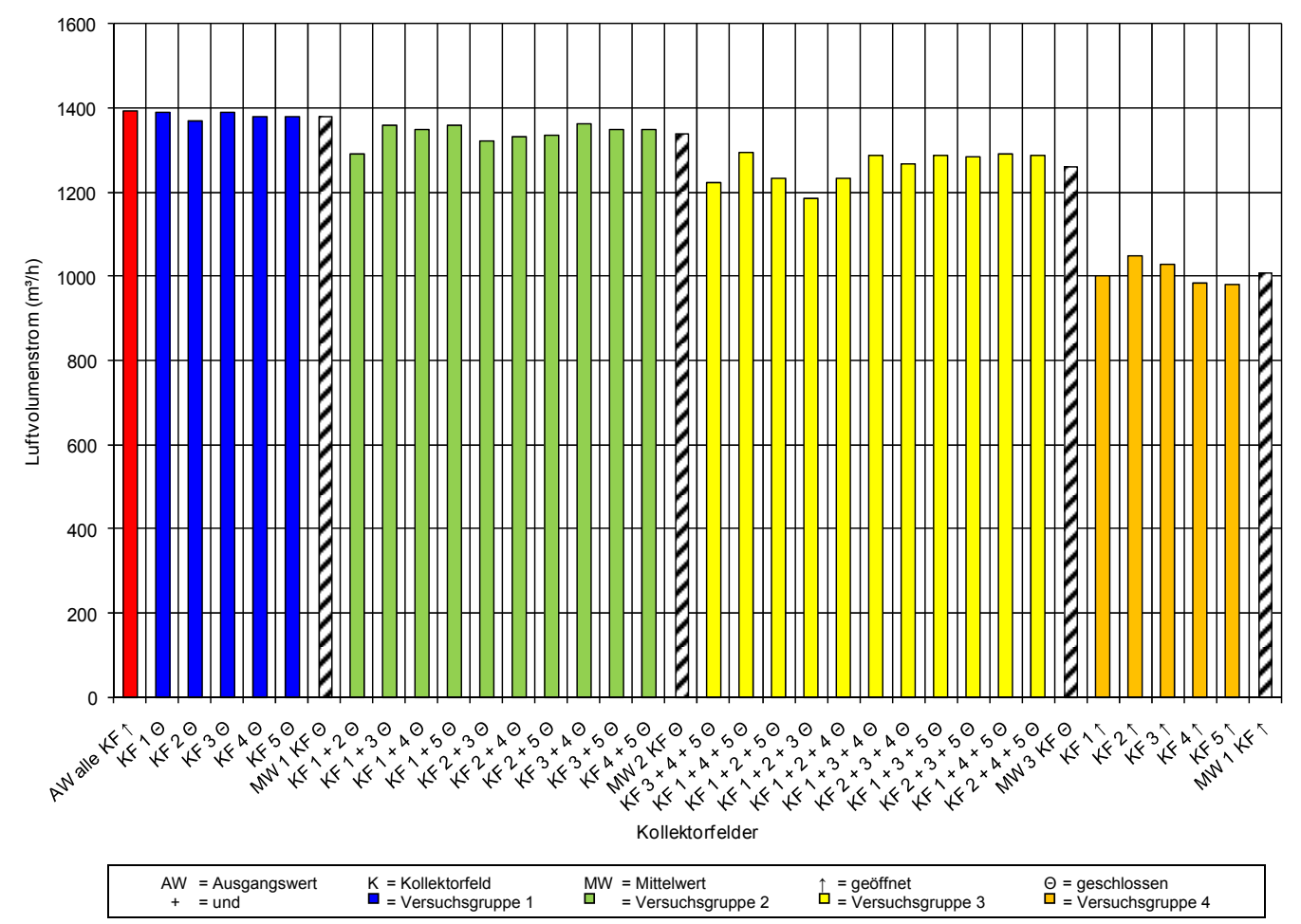

Abbildung 23: Geförderter Luftvolumenstrom der Kollektorfelder des Solardach-LuftKollektors mit Mittelwerten der einzelnen Versuchsgruppen

Tabelle 1: Mittlere Luftvolumenströme der Kollektorfelder

\begin{tabular}{lrrrrr}
\hline & alle $\mathrm{KF} \uparrow\left(\mathrm{m}^{3} / \mathrm{h}\right)$ & $1 \mathrm{KF} \Theta\left(\mathrm{m}^{3} / \mathrm{h}\right)$ & $2 \mathrm{KF} \Theta\left(\mathrm{m}^{3} / \mathrm{h}\right)$ & $3 \mathrm{KF} \Theta\left(\mathrm{m}^{3} / \mathrm{h}\right)$ & $1 \mathrm{KF} \uparrow\left(\mathrm{m}^{3} / \mathrm{h}\right)$ \\
\hline AW & 1392,69 & 1381,42 & 1340,07 & 1260,39 & 1009,34 \\
MW & & 7,76 & 21,69 & 36,16 & 29,14 \\
STAW & & & & & \\
\hline
\end{tabular}

AW, Ausgangswert; KF, Kollektorfeld; MW, Mittelwert;

STAW, Standardabweichung; $\uparrow$, geöffnet; $\ominus$, geschlossen.

Der Einfluss auf den geförderten Luftvolumenstrom $\left(\dot{V}_{\mathrm{L}}\right)$ wird in Abbildung 24 in Abhängigkeit von den untersuchten Faktoren dargestellt. Der Abbildung ist zu entnehmen, dass durch das Öffnen des Insektenschutzgitters (ISG) der geförderte Luftvolumenstrom selbst bei nur einem geöffneten Kollektorfeld (KF) unverändert bleibt. Weiterhin ist der Abbildung zu entnehmen, dass durch das Abtrennen der Kollektorfelder vor dem Wärmetauscher ebenfalls keine wesentliche Veränderung des Luftvolumenstromes eintritt. Eine deutliche Veränderung des geförderten Luftvolumenstromes tritt erst nach 
Abtrennung des Wärmetauschers mit Trichter (WT) auf. Anhand dieser Abbildung kann die Schlussfolgerung gezogen werden, dass das Insektenschutzgitter und die Kollektorfelder bei einem gleichzeitigen Betrieb der fünf Kollektorfelder keinen Einfluss auf den geförderten Luftvolumenstrom haben.

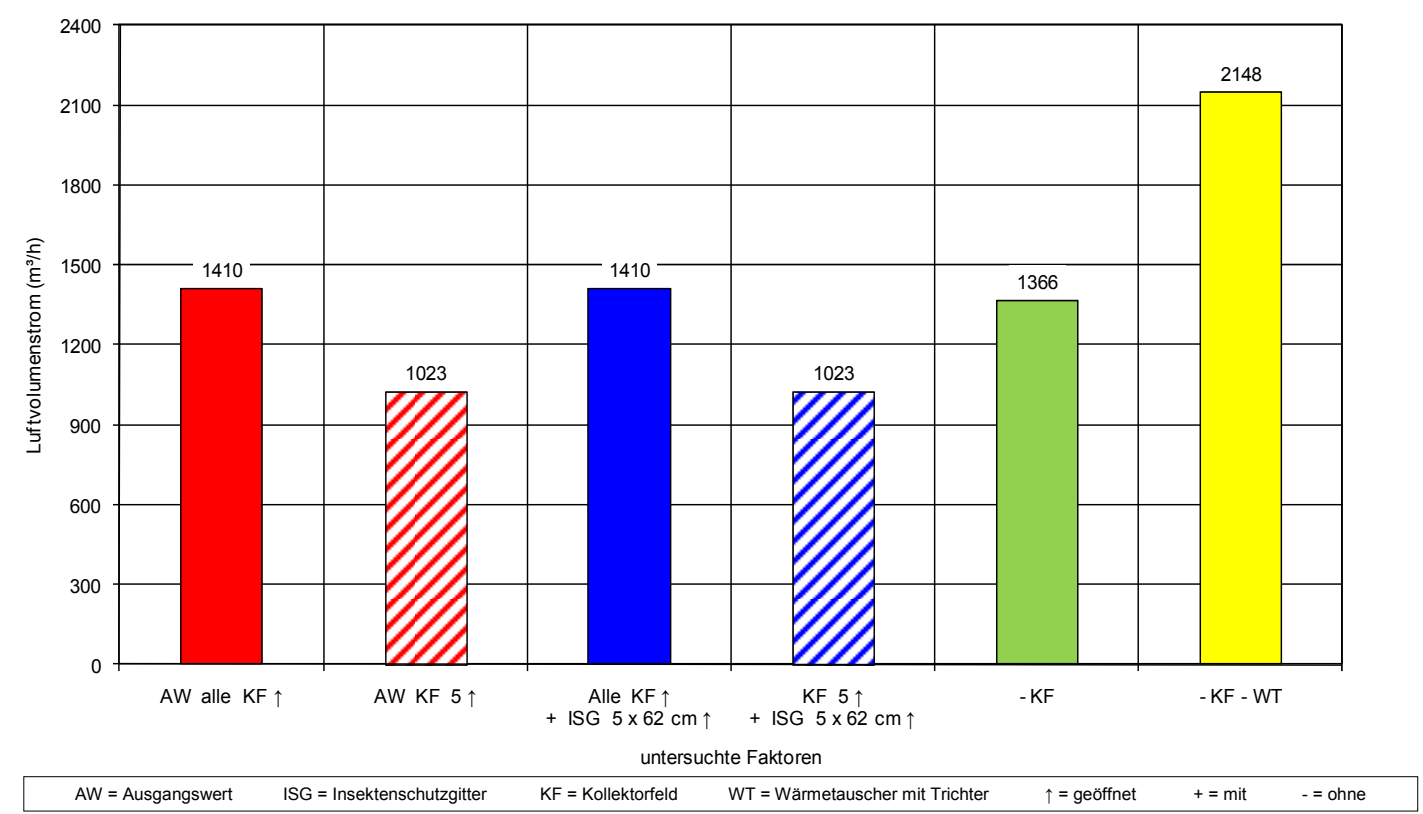

Abbildung 24: Einfluss der untersuchten Faktoren des Solardach-Luft-Kollektors auf den geförderten Luftvolumenstrom

\subsubsection{Luftgeschwindigkeiten}

Die errechneten Luftgeschwindigkeiten $\left(\mathrm{V}_{\mathrm{L}}\right)$ sind in Abbildung 25 in Abhängigkeit von dem jeweils betrachteten Anlagenabschnitt für den Zustand, dass alle Kollektorfelder (KF) geöffnet sind, dargestellt. Aus der Abbildung ist zu entnehmen, dass die niedrigsten Luftgeschwindigkeiten in den Kollektorfeldern mit rund $0,2 \mathrm{~m} / \mathrm{s}$ berechnet wurden. Die Luftgeschwindigkeit in dem Rohrsystem nach dem Wärmetauscher (RS nach WT) ist mit $15 \mathrm{~m} / \mathrm{s}$ doppelt so hoch wie die Luftgeschwindigkeit in den Rohren vor dem Wärmetauscher (RS1 u. RS2). Am Wärmetauscher (am WT) ergibt sich eine Luftgeschwindigkeit von rund $0,8 \mathrm{~m} / \mathrm{s}$. Diese Luftgeschwindigkeit entspricht der vierfachen Luftgeschwindigkeit der Kollektorfelder. Werden die 
Kollektorfelder und der Wärmetauscher mit dem Trichter (WT) von dem Rohrsystem abgetrennt, ergeben sich Luftgeschwindigkeiten von rund $11 \mathrm{~m} / \mathrm{s}$ in den Rohren vor dem Wärmetauscher (RS1 u. RS2).

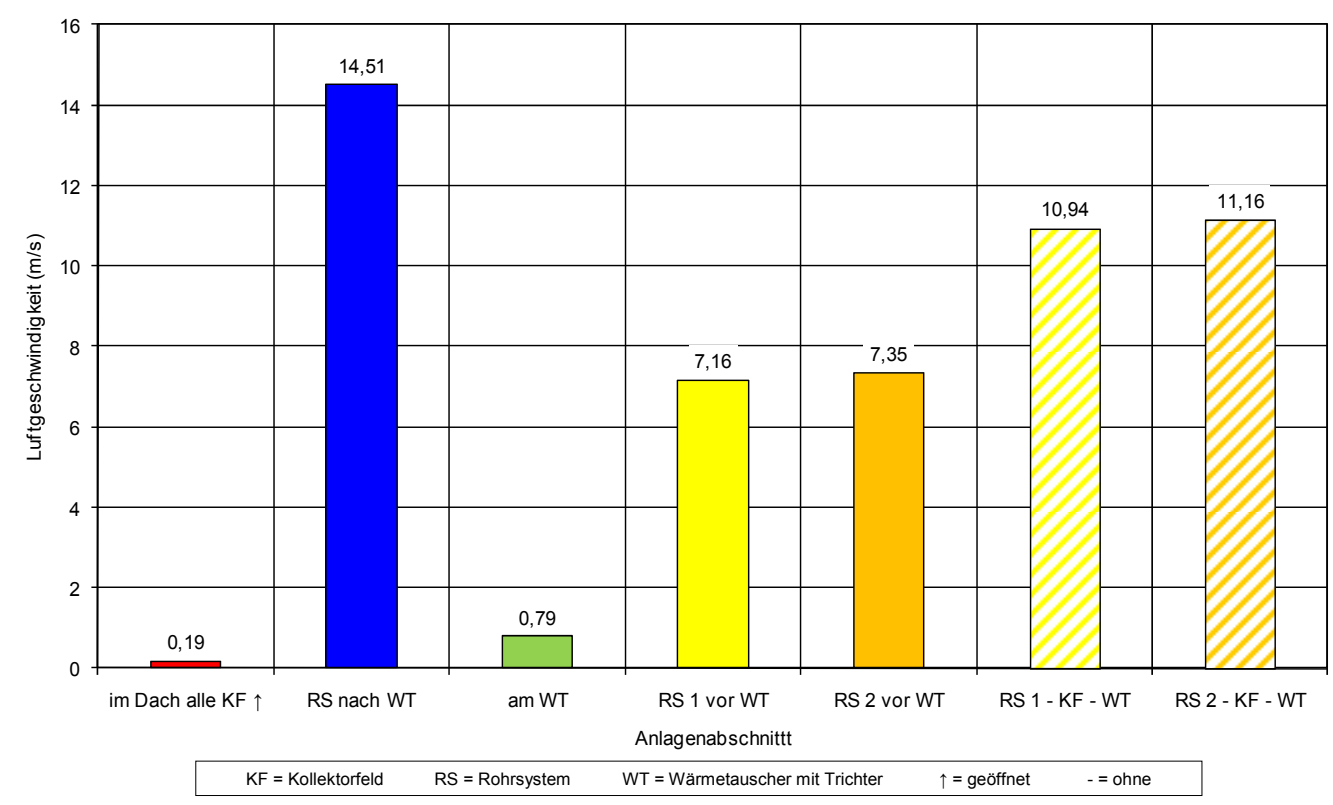

Abbildung 25: Luftgeschwindigkeiten in den Anlagenabschnitten des Solardach-LuftKollektors

In Abbildung 26 sind die errechneten Luftgeschwindigkeiten $\left(\mathrm{V}_{\mathrm{L}}\right)$ in Abhängigkeit von dem jeweils betrachteten Anlagenabschnitt für den Zustand, dass nur ein Kollektorfeld (KF) geöffnet ist, dargestellt. Die Abbildung zeigt, dass mit nur einem geöffneten Feld eine Luftgeschwindigkeit von rund $0,7 \mathrm{~m} / \mathrm{s}$ erzielt werden kann. Damit liegt die Luftgeschwindigkeit im Vergleich zur Abbildung 25 um 0,5 m/s höher. In dem Rohrsystem nach dem Wärmetauscher (RS nach WT) stellt sich eine Luftgeschwindigkeit von rund $11 \mathrm{~m} / \mathrm{s}$ ein. Diese Luftgeschwindigkeit liegt im Gegensatz zur berechneten Luftgeschwindigkeit aus Abbildung 25 um rund $4 \mathrm{~m} / \mathrm{s}$ niedriger. Am Wärmetauscher (am WT) fällt die Luftgeschwindigkeit von rund $0,8 \mathrm{~m} / \mathrm{s}$ auf rund $0,6 \mathrm{~m} / \mathrm{s}$. Die Luft in den Rohren vor dem Wärmetauscher (RS1 u. RS2) erreicht eine um $2 \mathrm{~m} / \mathrm{s}$ niedrigere Geschwindigkeit von rund $5 \mathrm{~m} / \mathrm{s}$. Nach der Abtrennung der Kollektorfelder und des Wärmetauschers (WT) ergeben sich 
die identischen Luftgeschwindigkeiten, die zuvor in Abbildung 25 ermittelt wurden. Der Vergleich mit Abbildung 25 zeigt, dass bei einem Kollektorbetrieb mit nur einem geöffneten Feld die Luftgeschwindigkeit im Kollektor steigt und die Luftgeschwindigkeit in dem folgenden Rohrsystem sinkt.

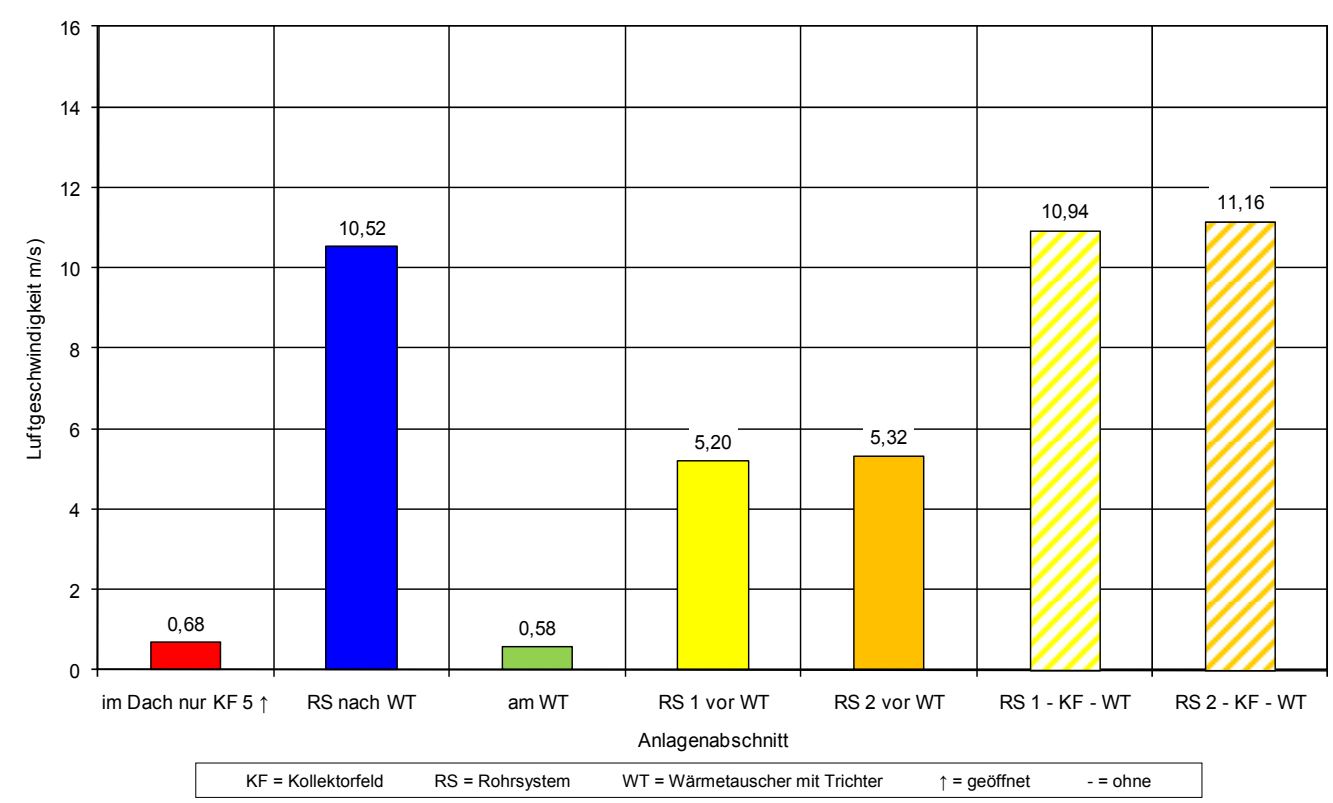

Abbildung 26: Luftgeschwindigkeiten in den unterschiedlichen Anlagenabschnitten bei nur einem geöffneten Kollektorfeld

\subsection{Berechnungen}

\subsubsection{Energetische Betrachtung des Daches}

Abbildung 27 stellt die zur Verfügung stehende thermische Nutzenergie des Kollektors bei einer Abkühlung der Lufttemperatur auf $10^{\circ} \mathrm{C}$ für die unterschiedlichen Monate im Jahresverlauf dar. In den Monaten Dezember, Januar und Februar liegen die Lufttemperaturen im Kollektor permanent unter $10^{\circ} \mathrm{C}$. In diesen Monaten ist kein Nutzenergiegewinn möglich. Im März und November ist fast kein Nutzen der Kollektorluft zu entziehen. Der Nutzenergiegewinn im Oktober liegt geringfügig über diesen beiden Monaten. Die zur Verfügung stehende Nutzenergie steigt von April bis August stetig an und erreicht im August ihren Höhepunkt. Im September fällt die Nutzenergie und 
erreicht ein Niveau knapp über dem Juni. Der Vergleich der Nutzenergie mit der bereinigten Nutzenergie zeigt, dass die Ventilatorantriebsenergie im Jahresverlauf leichten Schwankungen unterliegt. Die durchschnittlich aufgewendete Energie für die Ventilatoren betrug 7,62 \% der Nutzenergie. Zusammenfassend lässt sich feststellen, dass eine Verwertung der Kollektornutzenergie in den Monaten April bis Oktober möglich ist.

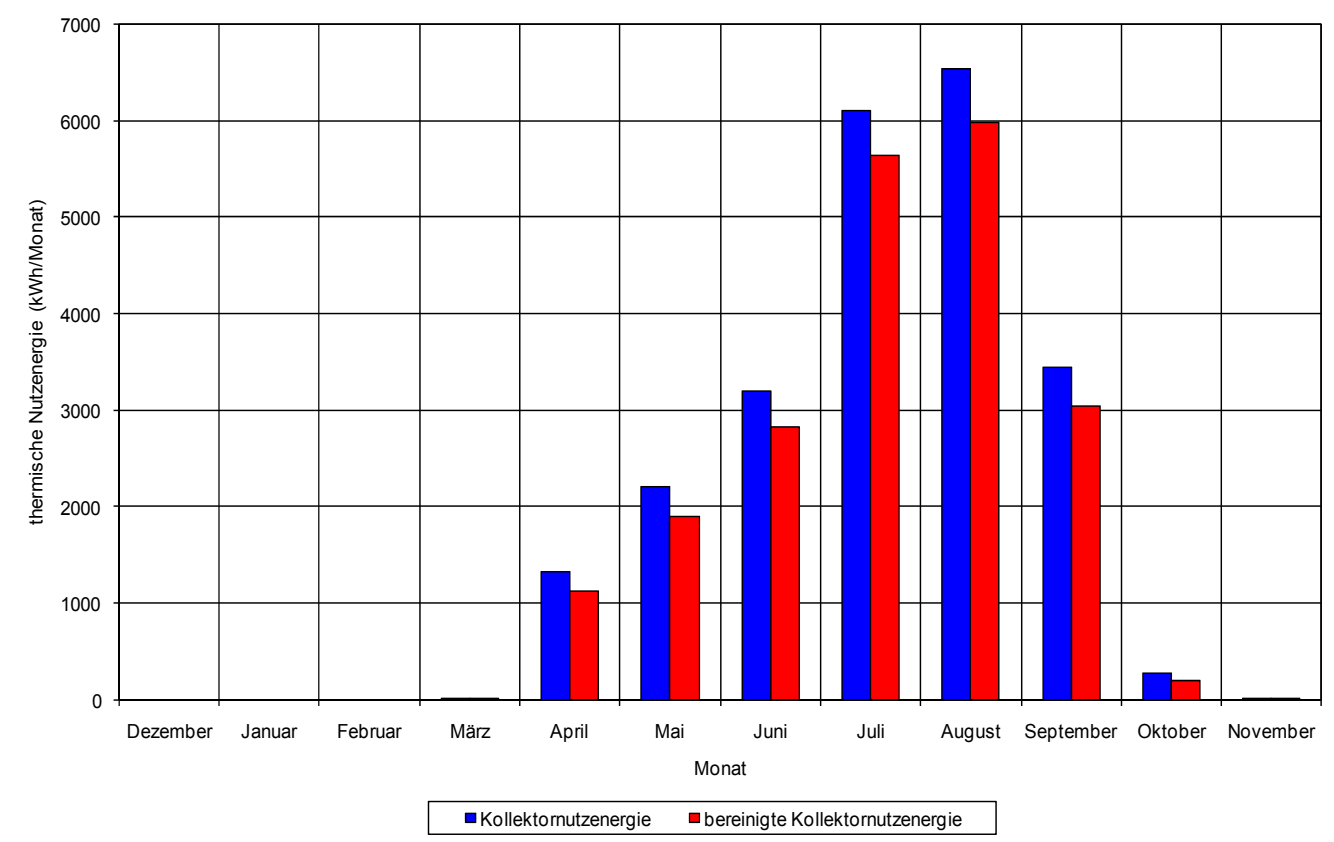

Abbildung 27: Thermische Nutzenergie des Solardach-Luft-Kollektors im Jahresverlauf bei einer Abkühlung der Lufttemperatur auf $10^{\circ} \mathrm{C}$

In Abbildung 28 sind die zur Verfügung stehende bereinigte thermische Energie und die bereinigte Nutzenergie der Umgebungsluft bei einer Abkühlung der Lufttemperatur auf $10^{\circ} \mathrm{C}$ für die unterschiedlichen Monate im Jahresverlauf dargestellt. Aus der Abbildung lässt sich feststellen, dass in den Monaten Dezember bis März keine Energie aus der Umgebungsluft entnommen werden kann. Vergleichbar mit Abbildung 27 steigt der Verlauf der zur Verfügung stehenden Nutzenergie in Abbildung 28 von April bis August stetig an und erreicht im August ihren Höhepunkt. Im September fällt die verfügbare Nutzenergie und erreicht ein deutlich höheres Nutzniveau als 
im Juni. Der Vergleich der absoluten Höhen mit Abbildung 27 zeigt, dass bei dem Ansaugen von Umgebungsluft deutlich niedrigere Nutzenergieniveaus erreicht werden. Bei der Betrachtung des Energiegehaltes der Umgebungsluft zeigt sich, dass durch die Nutzung niedriger Energiegehalte keine zusätzlichen Monate zur Energiegewinnung genutzt werden können (Abb. 28).

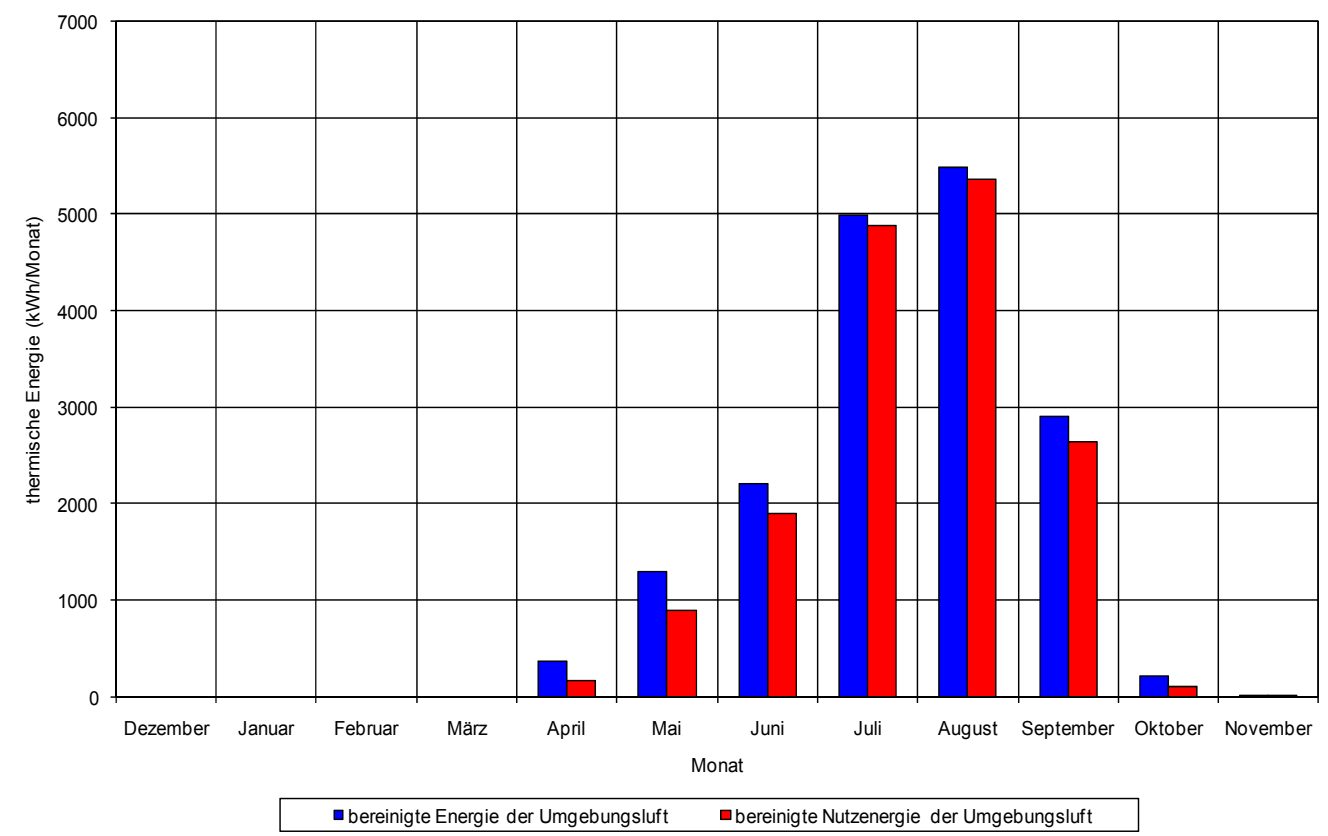

Abbildung 28: Bereinigter thermischer Energiegehalt der Umgebungsluft im Jahresverlauf bei einer Abkühlung der Lufttemperatur auf $10^{\circ} \mathrm{C}$

Die Abbildung 29 stellt den Nutzungszeitraum der bereinigten thermischen Nutzenergie des Kollektors und der Umgebungsluft bei einer Abkühlung der Lufttemperatur auf $10^{\circ} \mathrm{C}$ für die unterschiedlichen Monate im Jahresverlauf dar. Der Abbildung ist zu entnehmen, dass sich für die Monate April bis Juni und im Oktober bei der Kollektornutzenergie längere Nutzungszeiten ergeben. Für die Monate Juli bis September sind für die Kollektornutzenergie kürzere Nutzungszeiten zu entnehmen. Anhand der in Abbildung 27 und Abbildung 28 dargestellten thermischen Nutzenergien kann die Aussage getroffen werden, dass sich in den Monaten April bis Juni und im Oktober 
durch die Kollektornutzung ein längerer Nutzungszeitraum mit höheren thermischen Energien gegenüber der Umgebungsluft ergibt. Weiterhin kann die Aussage getroffen werden, dass in den Monaten Juli bis September bei kürzeren Nutzungszeiten höhere thermische Energien erzielt werden.

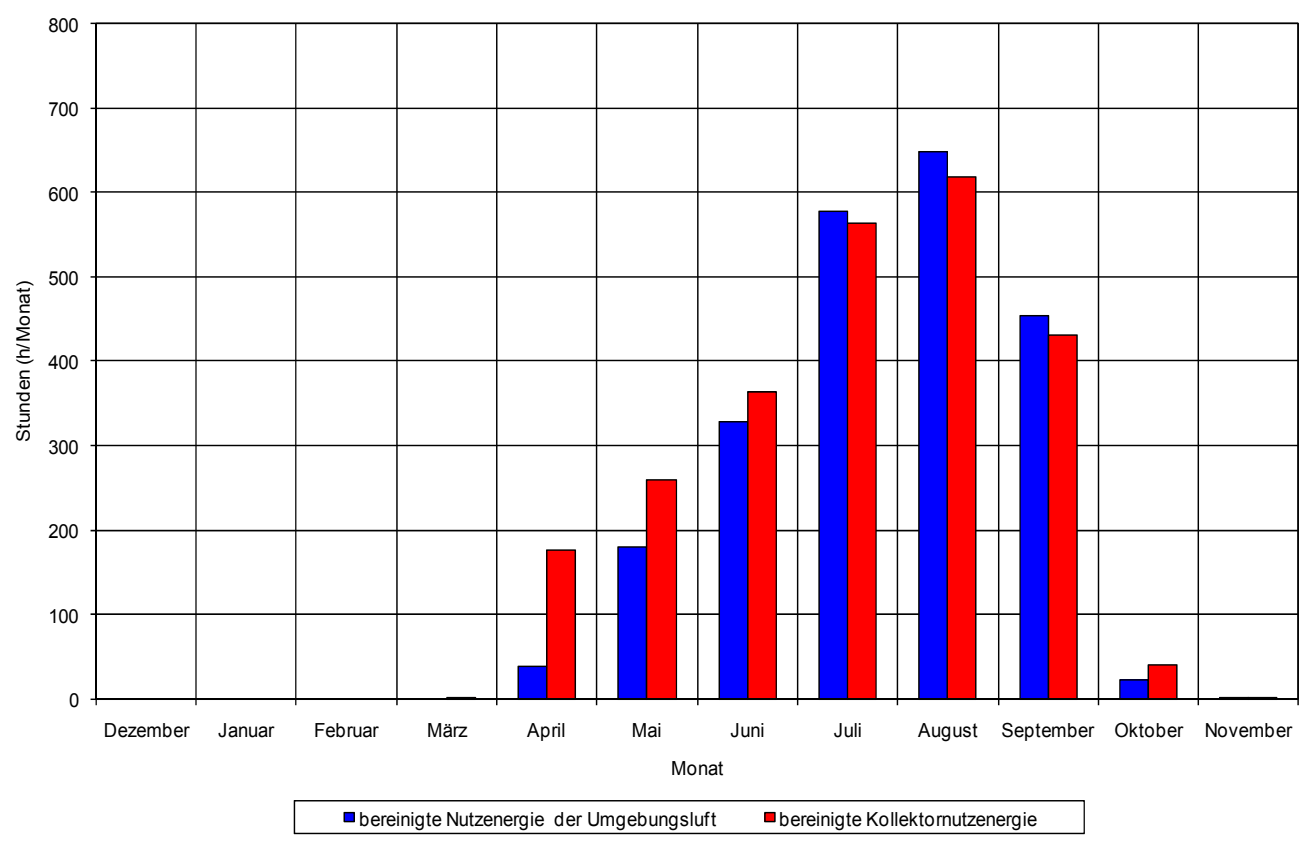

Abbildung 29: Nutzungszeitraum der bereinigten thermischen Nutzenergie des Solardach-Luft-Kollektors und der bereinigten thermischen Nutzenergie der Umgebungsluft bei einer Abkühlung der Lufttemperatur auf $10^{\circ} \mathrm{C}$

Die Abbildung 30 stellt die unterschiedlichen Energieanteile der bereinigten thermischen Nutzenergie des Kollektors bei einer Abkühlung der Lufttemperatur auf $10^{\circ} \mathrm{C}$ für verschiedene Monate im Jahresverlauf dar. Der Abbildung kann entnommen werden, dass sich das Verhältnis zwischen den Energieanteilen im Jahresverlauf verschiebt. Im April ist der durch den Kollektor gewonnene Energieanteil am höchsten. Der Energieanteil der Umgebungsluft nimmt von April bis Mai kontinuierlich zu, während der Anteil des Kollektors sinkt. Im September ist der Energieanteil des Kollektors im Verhältnis zur Umgebungsluft am geringsten. Die Energieanteile im Oktober sind dagegen relativ ausgeglichen. 


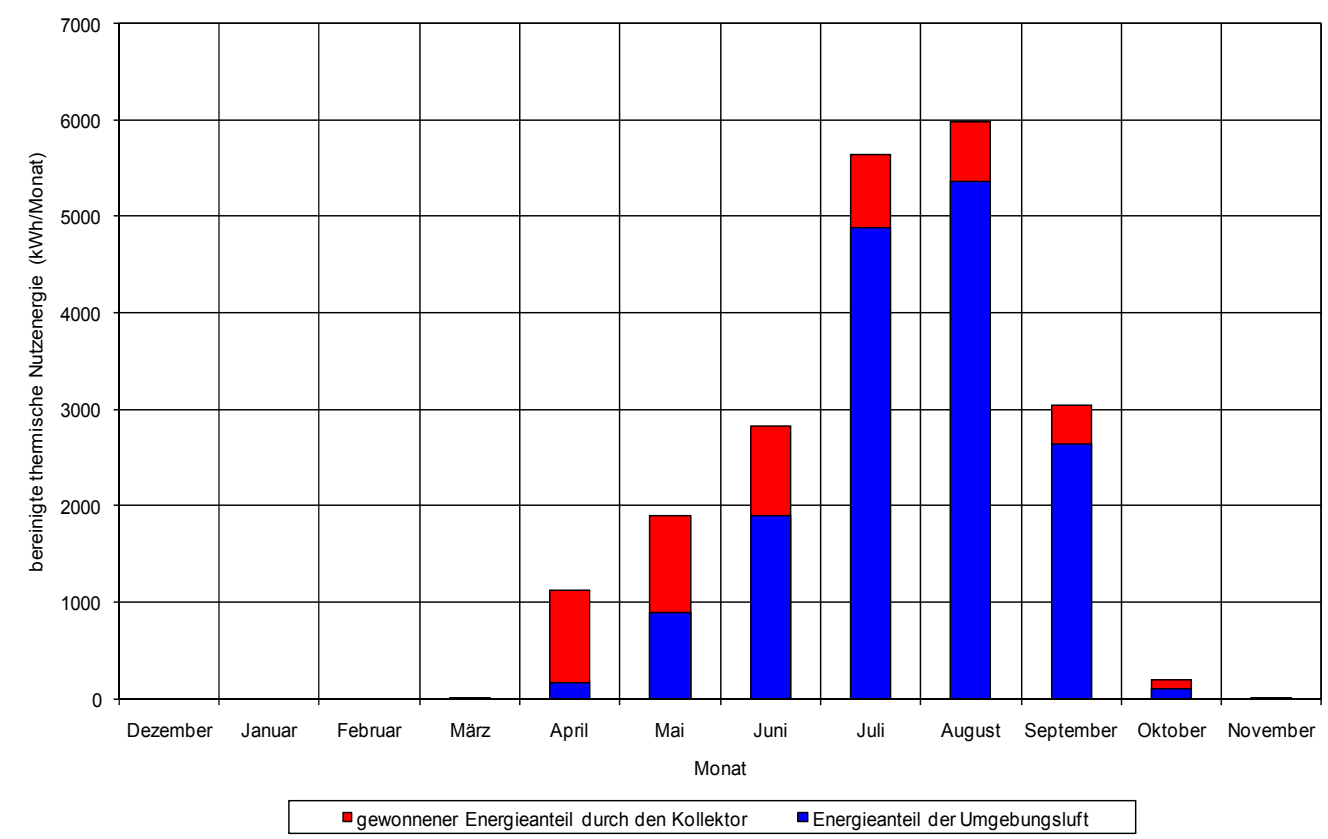

Abbildung 30: Energieanteile an der bereinigten thermischen Nutzenergie des Solardach-Luft-Kollektors im Jahresverlauf bei einer Abkühlung der Lufttemperatur auf $10^{\circ} \mathrm{C}$

In Abbildung 31 ist im Gegensatz zu Abbildung 30 die gesamte zusätzliche durch den Kollektor gewonnene Energie bei einer Abkühlung der Lufttemperatur auf $10^{\circ} \mathrm{C}$ für die verschiedenen Monate im Jahresverlauf dargestellt. Die Abbildung zeigt, dass der Kollektor über den gesamten Jahresverlauf einen thermischen Energiegewinn erzielt. Bei der Betrachtung der bereinigten Energie offenbart sich, dass in den Wintermonaten und im November ein nur sehr geringer Energiegewinn erzielt wird. Der Energiegewinn steigt von Dezember kontinuierlich an und erreicht im April seinen Höhepunkt. Besonders auffällig ist, dass der Energiegewinn in den Monaten Mai bis August konstant ist. Von September bis November nimmt der Energiegewinn wieder kontinuierlich ab. 


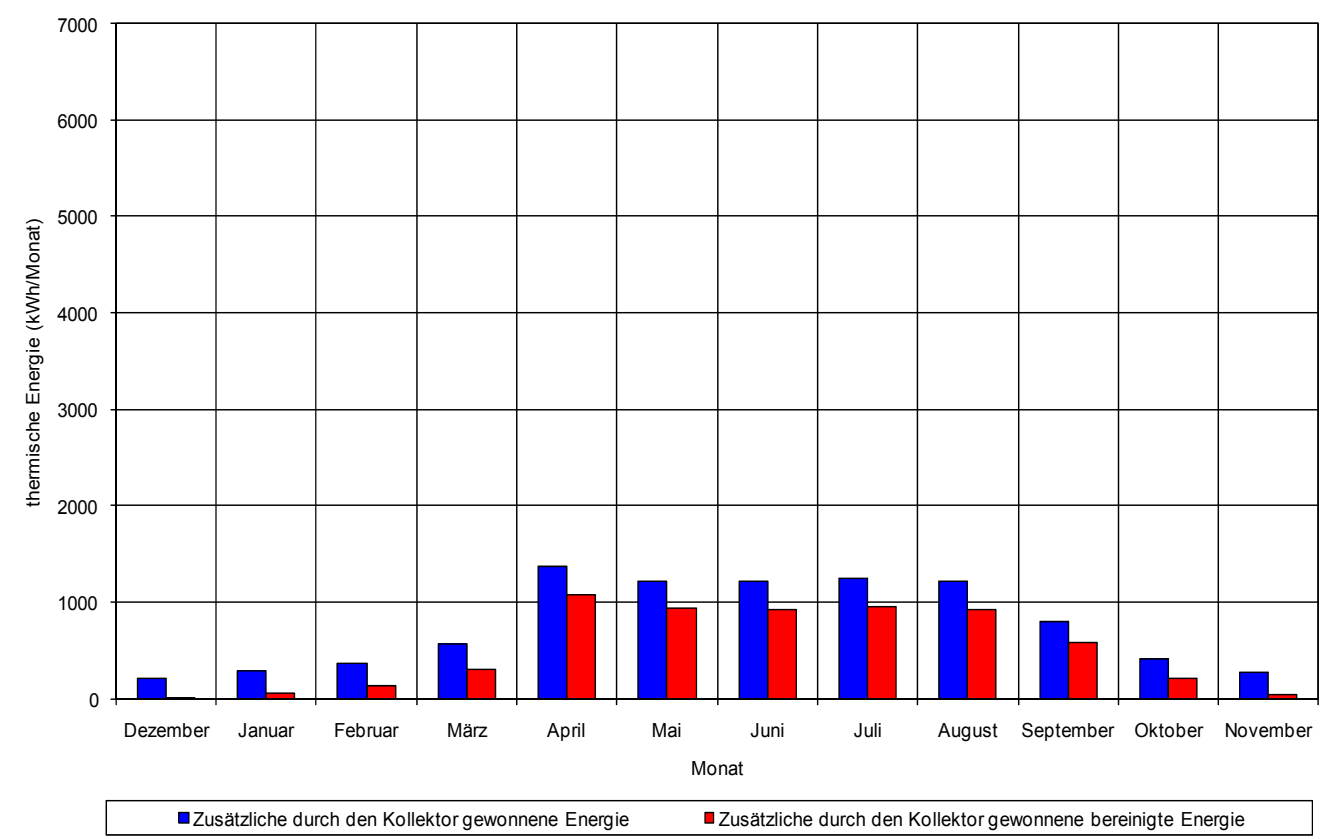

Abbildung 31: Zusätzlich gewonnene thermische Energie durch den Solardach-LuftKollektor ohne den Energiegehalt der Umgebungsluft im Jahresverlauf bei einer Abkühlung der Lufttemperatur auf $10^{\circ} \mathrm{C}$

Die Abbildung 32 zeigt die effiziente bereinigte Nutzenergie der Kollektorund Umgebungsluft bei einer Abkühlung der Lufttemperatur auf $10^{\circ} \mathrm{C}$ für die verschiedenen Monate im Jahresverlauf. Der Abbildung kann entnommen werden, dass es in den Monaten Juni bis September unter bestimmten Betriebsbedingungen effizienter ist, anstelle der Kollektorluft die Umgebungsluft zu nutzen. Im Juni ist die Nutzenergie aus der Umgebungsluft sehr gering. Dagegen ist für die Monate Juli bis August ein deutlicher Anstieg der Nutzenergie aus der Umgebungsluft abzulesen. Die höchste Nutzenergie wird der Umgebungsluft im August entzogen. Im September ist die Nutzenergie der Umgebungsluft geringer als im Juli. Die dargestellte Gesamtnutzenergie ergibt sich aus der Kollektornutzenergie und der Nutzenergie der Umgebungsluft. 


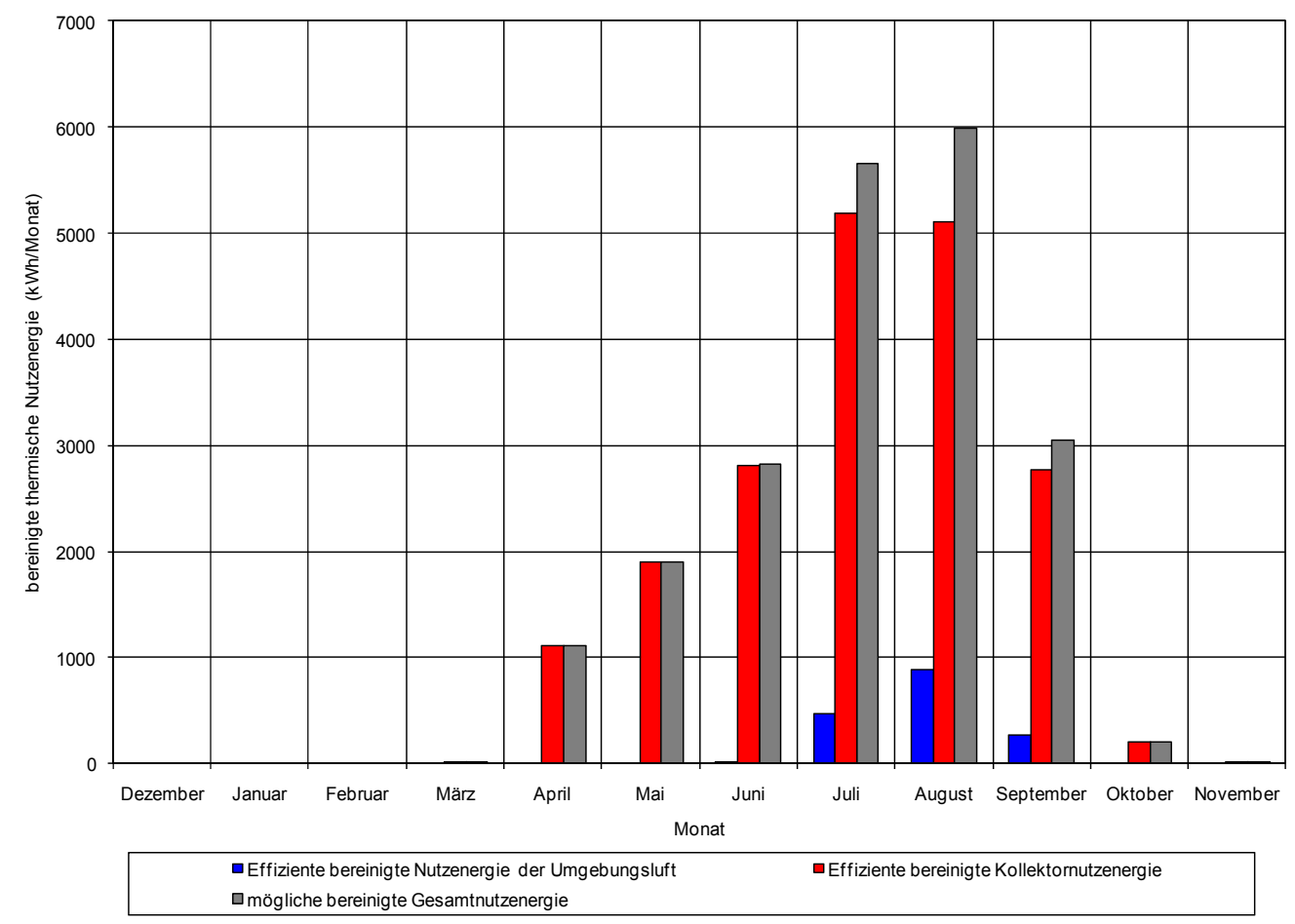

Abbildung 32: Bereinigte theoretisch mögliche Gesamtnutzenergie im Jahresverlauf

In Tabelle 2 sind die bereinigte Gesamtnutzenergie aus Abbildung 32 und die bereinigte Kollektornutzenergie aus Abbildung 27 gegenübergestellt. Die Gegenüberstellung zeigt, dass die Nutzungsdauer der Kollektornutzenergie im Mai und Juni mit rund 41 min länger ist als bei der Gesamtnutzenergie. Der Vergleich der Nutzenergien offenbart, dass in diesem Zeitraum eine höhere Nutzenergie von rund 2,8 kWh erzielt wurde. Der Tabelle kann weiterhin entnommen werden, dass bei der Gesamtnutzenergie (GNE) in den Monaten Juli bis September eine Energiedifferenz (ED) von rund $21 \mathrm{kWh}$ gegenüber der Kollektornutzenergie (KNE) erzielt wird. Die Tabelle zeigt, dass diese Energiedifferenz (ED) in einer kürzeren Nutzungszeit von $6 \mathrm{~h}$ und 42 min erzielt wurde. 
Tabelle 2: Vergleich der bereinigten möglichen Gesamtnutzenergie mit der bereinigten Kollektornutzenergie für verschiedene Monate

\begin{tabular}{|c|c|c|c|c|c|c|c|}
\hline Jahreszeit & Monat & GNE (kWh) & KNE (kWh) & ED (kWh) & NZGNE (h) & NZKNE (h) & DNZ (h) \\
\hline \multirow{3}{*}{ Winter } & Dezember & 0,00 & 0,00 & 0,00 & 0,00 & 0,00 & 0,00 \\
\hline & Januar & 0,00 & 0,00 & 0,00 & 0,00 & 0,00 & 0,00 \\
\hline & Februar & 0,00 & 0,00 & 0,00 & 0,00 & 0,00 & 0,00 \\
\hline \multirow{3}{*}{ Frühling } & März & 0,80 & 0,80 & 0,00 & 0,27 & 0,27 & 0,00 \\
\hline & April & 1117,49 & 1117,49 & 0,00 & 175,93 & 175,93 & 0,00 \\
\hline & Mai & 1895,83 & 1896,06 & $-0,23$ & 259,77 & 259,83 & $-0,06$ \\
\hline \multirow{3}{*}{ Sommer } & Juni & 2822,28 & 2824,82 & $-2,54$ & 362,90 & 363,53 & $-0,63$ \\
\hline & Juli & 5657,20 & 5646,21 & 10,99 & 560,37 & 562,77 & $-2,40$ \\
\hline & August & 5991,50 & 5987,21 & 4,29 & 615,03 & 618,43 & $-3,40$ \\
\hline \multirow{3}{*}{ Herbst } & September & 3048,93 & 3043,54 & 5,39 & 429,53 & 430,43 & $-0,90$ \\
\hline & Oktober & 200,15 & 200,15 & 0,00 & 39,90 & 39,90 & 0,00 \\
\hline & November & 4,31 & 4,31 & 0,00 & 0,07 & 0,07 & 0,00 \\
\hline
\end{tabular}

ED, Energiedifferenz; GNE, bereinigte Gesamtnutzenergie; NZGNE, Nutzungszeitraum bereinigte Gesamtnutzenergie; NZKNE, Nutzungszeitraum bereinigte Kollektornutzenergie; DNZ, Differenz Nutzungszeitraum; $\mathrm{KNE}$, bereinigte Kollektornutzenergie.

\subsubsection{Thermischer Energiebedarf der Jugendherberge}

Die Abbildung 33 stellt den thermischen Energiebedarf der Jugendherberge für die verschiedenen Monate im Jahresverlauf dar. Der Abbildung ist zu entnehmen, dass außer im Juli und August der höchste thermische Energiebedarf durch die Heizung der Jugendherberge verursacht wird. Dieser Energiebedarf steigt im Dezember und Januar an, fält im Februar und steigt im März wiederum an. Von März bis August fällt der Energiebedarf der Heizung in der Jugendherberge kontinuierlich. Im September steigt der Energiebedarf erneut bis zum Oktober an, bevor er im November leicht abfällt. Der Energiebedarf für die Brauchwassererwärmung ist von Januar bis März und im November relativ gering. Dieser geringe Energiebedarf steigt ab März kontinuierlich an und erreicht seinen Höhepunkt im Juli. Nach diesem Höhepunkt fällt der Energiebedarf bis zum Jahresende kontinuierlich ab. Auffällig ist, dass die Jugendherberge im Februar keinen Energiebedarf zur Brauchwassererwärmung hat. Zusammenfassend lässt sich feststellen, dass der Energiebedarf für die Brauchwassererwärmung in der Jugendherberge hauptsächlich von April bis Oktober besteht. Bei dem Vergleich der 
Abbildung 27 und Abbildung 33 zeigt sich, dass der Energiebedarf für die Brauchwassererwärmung der Jugendherberge und der mögliche Verwertungszeitraum der Kollektornutzenergie zusammenfallen.

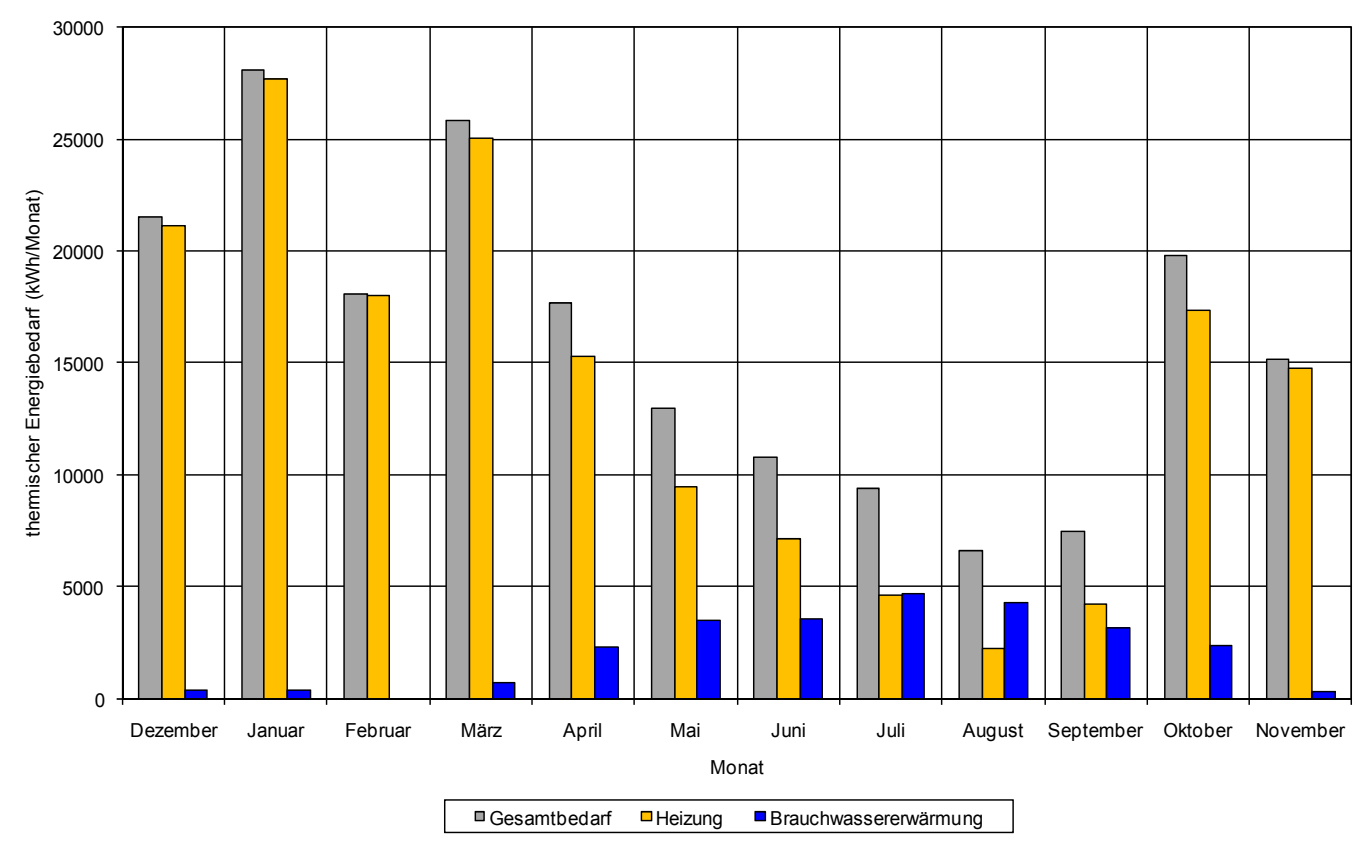

Abbildung 33: Thermischer Energiebedarf der Jugendherberge im Jahresverlauf 2008/2009

\subsubsection{Simulation der Wärmetauschertemperaturen}

Wie zuvor in Abbildung 27 dargestellt, ist in den Wintermonaten keine Nutzung des Kollektors möglich. In Tabelle 3 sind die berechneten Wärmetauschertemperaturen für die Jahreszeiten Frühling, Sommer und Herbst dargestellt. Die durchschnittliche Lufteintrittstemperatur (LET) in den Wärmetauscher ist im Frühling und Sommer annähernd identisch. Im Vergleich zum Frühling ist die Lufteintrittstemperatur im Herbst um rund $4 \mathrm{~K}$ niedriger. Die durchschnittliche relative Luftfeuchte (rLF) ist im Sommer mit $80 \%$ am höchsten. Diese relative Luftfeuchte fällt im Frühling um $1 \%$ und im Herbst um $2 \%$. Im Frühling beträgt der geförderte Luftvolumenstrom (LVS) $1314 \mathrm{~m}^{3} / \mathrm{h}$. Ausgehend von diesem Luftvolumenstrom sinkt im Sommer der Volumenstrom um $9 \mathrm{~m}^{3} / \mathrm{h}$ und steigt um diesen Volumenstrom im Herbst. Die 
Luftaustrittstemperaturen (LAT) aus dem Wärmetauscher liegen ungefähr zwischen $11^{\circ} \mathrm{C}$ und $14{ }^{\circ} \mathrm{C}$. Die Solevorlauftemperatur (SVT) ist bei allen Jahreszeiten gleich. Bei der Solerücklauftemperatur (SRT) wird mit einer Solespreizung (SSP) von rund $8 \mathrm{~K}$ im Sommer die höchste Soletemperatur erzielt. Mit einer Spreizung von $4 \mathrm{~K}$ ist die Soletemperatur im Frühling am niedrigsten. Die Werte der Sole im Herbst liegen im Gegensatz zum Frühling um rund $2 \mathrm{~K}$ über diesen Werten. Für den Herbst und Frühling ergeben sich annähernd identische Laufzeiten (LZ). Die Laufzeit des Wärmetauschers im Sommer ist im Gegensatz zum Frühling und Herbst mehr als dreimal so hoch.

Tabelle 3: Simulierte Wärmetauschertemperaturen für verschiedene Jahreszeiten

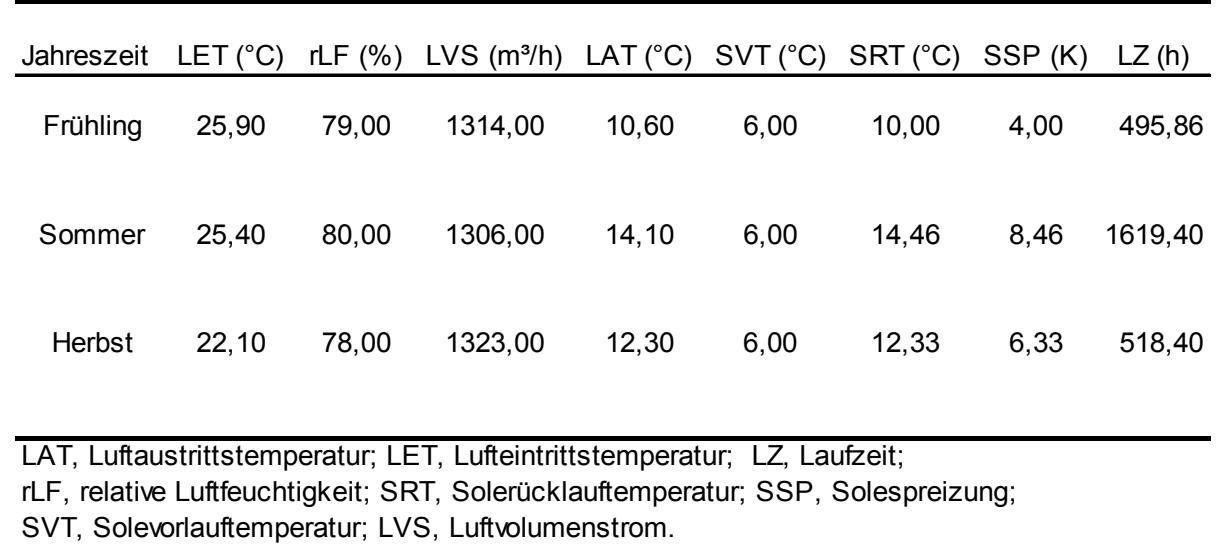

\subsubsection{Simulation des Wärmepumpenbetriebes}

\subsubsection{Betrieb bei vollständiger Wärmeabnahme}

Die Tabelle 4 zeigt die Leistungszahlen des Verdichters $\left(\varepsilon_{\mathrm{V}}\right)$ bei verschiedenen Temperaturniveaus auf der warmen Seite der Wärmepumpe (kPS, kältester Pufferspeicher) für die unterschiedlichen Jahreszeiten. Neben den Leistungszahlen wurden die Verdampferleistung (VL), die elektrische Antriebsleistung des Verdichters (EAL) und die Wärmeleistung des Verdichters (WL) dargestellt. Der Tabelle ist $\mathrm{zu}$ entnehmen, dass die 
Verdampferleistung und die Wärmeleistung des Verdichters mit der Zunahme des Temperaturniveaus auf der warmen Seite sinken und die Elektroantriebsleistung steigt. Die Tabelle zeigt weiterhin, dass die Leistungszahlen des Verdichters mit der Zunahme des Temperaturniveaus auf der warmen Seite stetig abnehmen. Der Vergleich der Jahreszeiten zeigt, dass im Sommer und Herbst die höchsten Leistungszahlen erzielt werden. Es ist festzustellen, dass im Frühling bei einem Temperaturniveau von $55^{\circ} \mathrm{C}$ auf der warmen Seite kein Betrieb der Wärmepumpe möglich ist. Die Gegenüberstellung der Soletemperaturen aus der Tabelle 3 mit dem Temperaturniveau auf der warmen Seite des Verdichters zeigt: Je kleiner die Differenz zwischen der warmen (kPS) und kalten Seite (SRT) ist, umso höher ist die Leistungszahl. 
Tabelle 4: Leistungszahlen des Verdichters für verschiedene Jahreszeiten und Temperaturniveaus

\begin{tabular}{|c|c|c|c|c|c|}
\hline Jahreszeit & $\operatorname{kPS}\left({ }^{\circ} \mathrm{C}\right)$ & VL (kW) & EAL $(\mathrm{kW})$ & WL (kW) & $\varepsilon_{\mathrm{v}}$ \\
\hline Frühling & & 5,27 & 1,30 & 6,57 & 5,05 \\
\hline Sommer & 30 & 6,76 & 1,28 & 8,04 & 6,28 \\
\hline Herbst & & 6,75 & 1,28 & 8,03 & 6,27 \\
\hline Frühling & & 4,83 & 1,52 & 6,35 & 4,18 \\
\hline Sommer & 35 & 6,28 & 1,50 & 7,78 & 5,19 \\
\hline Herbst & & 6,27 & 1,50 & 7,77 & 5,18 \\
\hline Frühling & & 4,38 & 1,80 & 6,18 & 3,43 \\
\hline Sommer & 40 & 5,79 & 1,76 & 7,55 & 4,29 \\
\hline Herbst & & 5,78 & 1,76 & 7,54 & 4,28 \\
\hline Frühling & & 3,92 & 2,12 & 6,04 & 2,85 \\
\hline Sommer & 45 & 5,27 & 2,07 & 7,34 & 3,55 \\
\hline Herbst & & 5,27 & 2,07 & 7,34 & 3,55 \\
\hline Frühling & & 3,43 & 2,48 & 5,91 & 2,38 \\
\hline Sommer & 50 & 4,74 & 2,43 & 7,17 & 2,95 \\
\hline Herbst & & 4,74 & 2,43 & 7,17 & 2,95 \\
\hline Frühling & & 0,00 & 0,00 & 0,00 & 0,00 \\
\hline Sommer & 55 & 4,20 & 2,83 & 7,03 & 2,48 \\
\hline Herbst & & 4,19 & 2,83 & 7,02 & 2,48 \\
\hline
\end{tabular}

In Tabelle 5 sind die Arbeitszahlen $(\beta)$ bei verschiedenen Temperaturniveaus auf der warmen Seite der Wärmepumpe (kPS) für die unterschiedlichen Jahreszeiten dargestellt. Neben den Arbeitszahlen bei einer vollständigen Wärmeabnahme wurden die Wärmepumpenlaufzeiten (WLZ), die abgegebene Wärmemenge (aWM) und die elektrische Antriebsenergie (eAE) angegeben. Die anhand der Leistungszahl $\left(\varepsilon_{\mathrm{V}}\right)$ beschriebenen grundlegenden Zusammenhänge wiederholen sich in Tabelle 5. Der Vergleich mit den Leistungszahlen des Verdichters aus Tabelle 4 zeigt, dass die Arbeitszahlen der Wärmepumpe deutlich unter den Leistungszahlen des Verdichters liegen. 
Tabelle 5: Arbeitszahlen bei vollständiger Wärmeabnahme für verschiedene Jahreszeiten und Temperaturniveaus

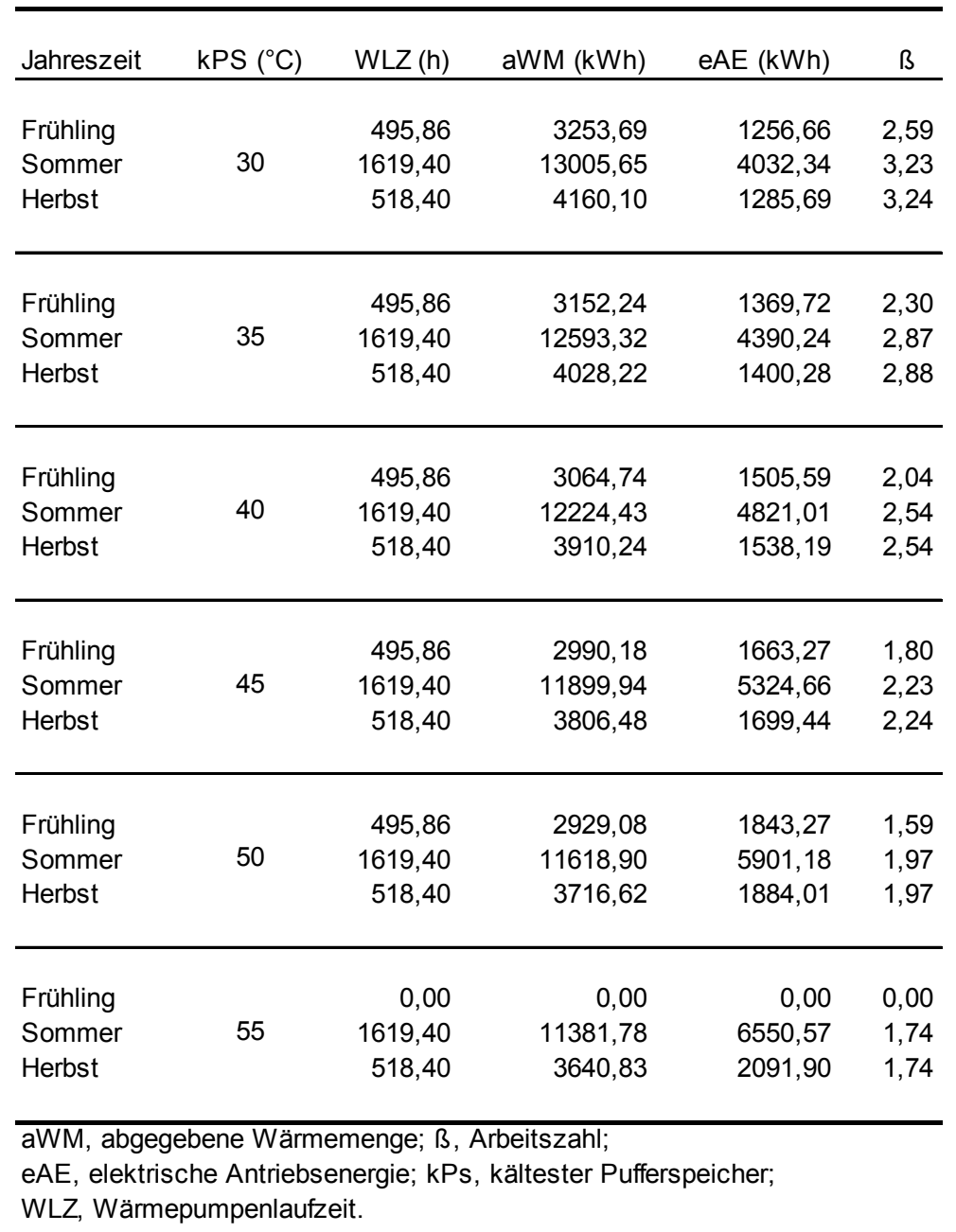

Die Tabelle 6 zeigt die Jahresarbeitszahlen $\left(\beta_{a}\right)$ bei verschiedenen Temperaturniveaus auf der warmen Seite der Wärmepumpe (kPS). Neben den Jahresarbeitszahlen bei einer vollständigen Wärmeabnahme wurden die Wärmepumpenlaufzeiten (WLZ), die abgegebene Wärmemenge (aWM) und die elektrische Antriebsenergie (eAE) dargestellt. Der Tabelle ist zu entnehmen, dass die Wärmepumpe je nach Temperaturniveau eine Laufzeit von $2.138 \mathrm{~h}$ und $2.634 \mathrm{~h}$ erreicht. Innerhalb dieser Laufzeit erlangt die Wärmepumpe je nach Temperaturniveau eine Jahresarbeitszahl zwischen 1,74 und 3,11. Die temperaturabhängige abgegebene Wärmemenge beläuft sich zwischen $20.419 \mathrm{kWh}$ und $15.023 \mathrm{kWh}$. Die aufgenommene elektrische 
Antriebsenergie bewegt sich von $6.575 \mathrm{kWh}$ im Minimum bis hin zu einem Maximum von 8.642 kWh. Zusammenfassend kann gesagt werden, dass die längste Wärmepumpenlaufzeit mit der höchsten Jahresarbeitszahl und der größten abgegebenen Wärmemenge bei einer Temperatur auf der warmen Seite von $30{ }^{\circ} \mathrm{C}$ mit der geringsten elektrischen Antriebsenergie erzielt wird.

Tabelle 6: Jahresarbeitszahlen bei vollständiger Wärmeabnahme für verschiedene Temperaturniveaus

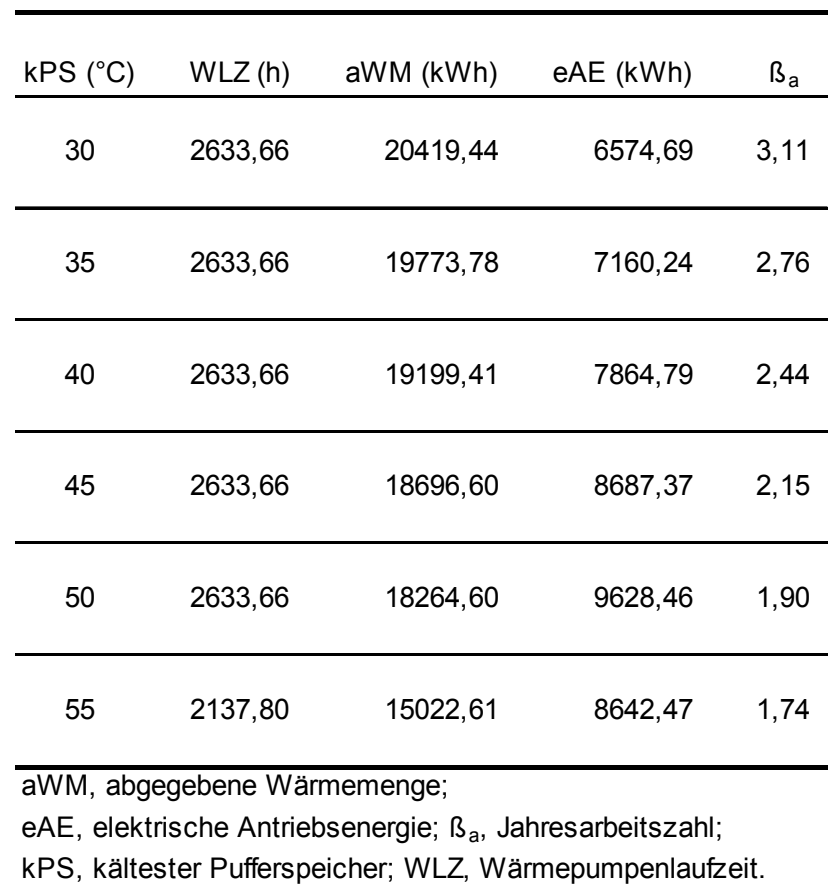

\subsubsection{Betrieb bezogen auf die Wärmeabnahme in der Jugendherberge}

In der Tabelle 7 sind für die einzelnen Monate im Jahresverlauf die Übernachtungen (ÜN) in der Jugendherberge und der temperaturbezogene Wasserverbrauch dargestellt. Der Tabelle kann entnommen werden, dass die $60^{\circ} \mathrm{C}$ warme Mischwassermenge (MWV $60{ }^{\circ} \mathrm{C}$ ) nur noch $60 \%$ der angenommenen $40^{\circ} \mathrm{C}$ warmen Wassermenge (WWV $40{ }^{\circ} \mathrm{C}$ ) ausmacht. Die benötigte Wassermenge wird durch Zumischen von $40 \% 10{ }^{\circ} \mathrm{C}$ kalten Wassers (MWV $10^{\circ} \mathrm{C}$ ) erreicht. Anhand dieser Tabelle lässt sich feststellen, 
dass das Temperaturniveau bei der Brauchwassererwärmung in der Jugendherberge um $20 \mathrm{~K}$ höher ist und dass sich dadurch die Warmwassermenge (MWV $60{ }^{\circ} \mathrm{C}$ ) auf $60 \%$ reduziert.

Tabelle 7: Warmwasserverbrauch in der Jugendherberge 2008/2009

\begin{tabular}{rlrrrr}
\hline \multirow{2}{*}{ Jahreszeit } & Monat & ÜN & WWV $40{ }^{\circ} \mathrm{C}(\mathrm{I})$ & MWV 60 ${ }^{\circ} \mathrm{C}(\mathrm{I})$ & MWV $10{ }^{\circ} \mathrm{C}(\mathrm{I})$ \\
\hline \multirow{3}{*}{ Winter } & Dezember & 321 & 9951,00 & 5970,60 & 3980,40 \\
& Januar & 346 & 10726,00 & 6435,60 & 4290,40 \\
& Februar & 0 & 0,00 & 0,00 & 0,00 \\
\hline \multirow{3}{*}{ Frühling } & März & 660 & 20460,00 & 12276,00 & 8184,00 \\
& April & 2113 & 65503,00 & 39301,80 & 26201,20 \\
& Mai & 3209 & 99479,00 & 59687,40 & 39791,60 \\
\hline \multirow{2}{*}{ Sommer } & Juni & 3317 & 102827,00 & 61696,20 & 41130,80 \\
& Juli & 4339 & 134509,00 & 80705,40 & 53803,60 \\
& August & 3983 & 123473,00 & 74083,80 & 49389,20 \\
\hline \multirow{2}{*}{ Herbst } & September & 2949 & 91419,00 & 54851,40 & 36567,60 \\
& Oktober & 2190 & 67890,00 & 40734,00 & 27156,00 \\
& November & 314 & 9734,00 & 5840,40 & 3893,60
\end{tabular}

MWV, Mischwasserverbrauch; ÜN, Übernachtungen; WWV, Warmwasserverbrauch.

Die Abbildung 34 zeigt die abgenommene Wärmemenge der Jugendherberge für die verschiedenen Monate im Jahresverlauf. Der Abbildung ist zu entnehmen, dass im Februar keine und in den Monaten Dezember, Januar und November nur eine sehr geringe Energieabnahme erfolgt. Ab März steigt die Energieabnahme der Jugendherberge stetig an und erreicht ihren Höhepunkt im Juli. Im August beginnend, fällt die Energieabnahme der Jugendherberge stetig bis zum Jahresende. Anhand der Abbildung 34 lässt sich feststellen, dass die Hauptenergieabnahme der Jugendherberge in den Monaten April bis Oktober liegt. 


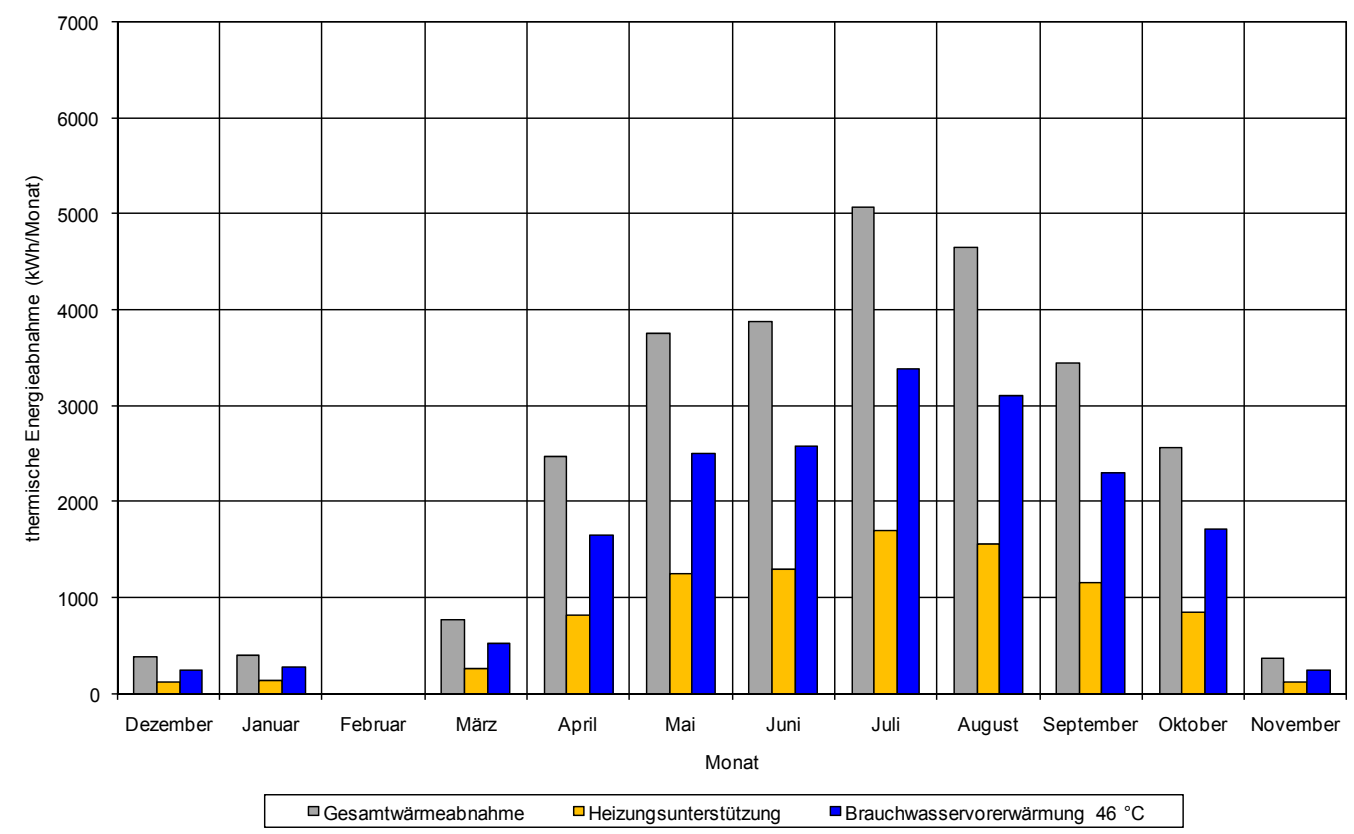

Abbildung 34: Abgenommene Wärmemenge der Jugendherberge 2008/2009

Die Darstellungen der folgenden Ergebnisse beziehen sich aufgrund der höheren Arbeitszahl ( $\beta$ ) nur auf die niedrigste Temperatur der warmen Seite der Wärmepumpe (kPS). In Tabelle 8 sind die abgegebene Wärmemenge (aWM) bei einem Temperaturniveau von $30{ }^{\circ} \mathrm{C}$ auf der warmen Seite der Wärmepumpe und die Gesamtwärmeabnahme der Jugendherberge (GWA) für die einzelnen Monate im Jahresverlauf gegenübergestellt. Weiterhin sind der Tabelle die Wärmepumpenlaufzeiten (WLZ) und die Wärmebedarfsdeckungen (WBD) zu entnehmen. Aus der Tabelle ist abzulesen, dass in den Wintermonaten die Gesamtwärmeabnahme der Jugendherberge einem bereits in Abbildung 27 dargestellten Energiemangel der Kollektorluft gegenübersteht. Dies bedeutet, dass trotz einer möglichen Energieabnahme keine Wärmepumpenlaufzeit realisiert werden kann. Der Tabelle ist weiterhin zu entnehmen, dass bis auf die Monate August und September die abgegebene Wärmemenge unter der möglichen Gesamtwärmeabnahme der Jugendherberge liegt. 
Für die Monate August und September kann dagegen abgelesen werden, dass die Wärmepumpe aufgrund einer geringeren Energieabnahme nicht nach ihren Möglichkeiten betrieben werden kann.

Tabelle 8: Wärmepumpenlaufzeiten bezogen auf die Wärmeabnahme in der Jugendherberge für verschiedene Jahreszeiten

\begin{tabular}{rlrrrrr}
\hline \multirow{2}{*}{ Jahreszeit } & Monat & $\mathrm{mWLZ}(\mathrm{h})$ & $\mathrm{aWM} 30^{\circ} \mathrm{C}(\mathrm{kWh})$ & $\mathrm{GWA}(\mathrm{kWh})$ & WBD $(\mathrm{kWh})$ & WLZ $(\mathrm{h})$ \\
\hline \multirow{2}{*}{ Winter } & Dezember & 0,00 & 0,00 & 374,97 & $-374,97$ & 0,00 \\
& Januar & 0,00 & 0,00 & 404,18 & $-404,18$ & 0,00 \\
& Februar & 0,00 & 0,00 & 0,00 & 0,00 & 0,00 \\
\hline \multirow{2}{*}{ Frühling } & März & 3,30 & 21,66 & 770,96 & $-749,30$ & 3,30 \\
& April & 197,03 & 1292,88 & 2468,24 & $-1175,36$ & 197,03 \\
& Mai & 295,53 & 1939,21 & 3748,49 & $-1809,28$ & 295,53 \\
\hline \multirow{2}{*}{ Sommer } & Juni & 396,30 & 3182,75 & 3874,65 & $-691,90$ & 396,30 \\
& Juli & 578,03 & 4642,27 & 5068,46 & $-426,19$ & 578,03 \\
& August & 645,07 & 5180,63 & 4652,61 & 528,02 & 579,32 \\
\hline \multirow{2}{*}{ Herbst } & September & 459,27 & 3685,56 & 3444,78 & 240,78 & 429,26 \\
& Oktober & 55,13 & 442,44 & 2558,18 & $-2115,74$ & 55,13 \\
& November & 4,00 & 32,10 & 366,80 & $-334,70$ & 4,00
\end{tabular}

aWM; abgegebene Wärmemenge; WBD, Wärmebedarfsdeckung; GWA, Gesamtwärmeabnahme; mWLZ, mögliche Wärmepumpenlaufzeit; WLZ, Wärmepumpenlaufzeit.

Die Tabelle 9 zeigt die Arbeitszahlen $(\beta$ ) bei einem Temperaturniveau von $30{ }^{\circ} \mathrm{C}$ auf der warmen Seite der Wärmepumpe (kPS) für die unterschiedlichen Jahreszeiten. Neben den Arbeitszahlen für die Energieabnahme der Jugendherberge wurden die Wärmepumpenlaufzeiten (WLZ), die abgegebene Wärmemenge (aWM) und die elektrische Antriebsenergie (eAE) dargestellt. Der Vergleich mit der Tabelle 5 zeigt, dass sich durch die verringerte Laufzeit (WLZ) im Sommer und Herbst die abgegebene Wärmemenge (aWM) und die elektrische Antriebsenergie (eAE) im gleichen Verhältnis verringert haben. Demzufolge stimmen die Arbeitszahlen bei der Energieabnahme der Jugendherberge mit den Arbeitszahlen bei einer vollständigen Energieabnahme überein. 
Tabelle 9: Arbeitszahlen bezogen auf die Wärmeabnahme in der Jugendherberge für verschiedene Jahreszeiten

\begin{tabular}{lrrrrr}
\hline & & & & & \\
Jahreszeit & $\mathrm{kPS}\left({ }^{\circ} \mathrm{C}\right)$ & $\mathrm{WLZ}(\mathrm{h})$ & $\mathrm{aWM}(\mathrm{kWh})$ & $\mathrm{eAE}(\mathrm{kWh})$ & $\beta$ \\
\hline & & & & & \\
Frühling & \multirow{2}{*}{30} & 1553,86 & 3253,69 & 1256,66 & 2,59 \\
Sommer & & 488,39 & 12477,60 & 3868,62 & 3,23 \\
Herbst & & 3919,27 & 1211,26 & 3,24
\end{tabular}

aWM, abgegebene Wärmemenge; $ß$, Arbeitszahl;

eAE, elektrische Antriebsenergie; kPs, kältester Pufferspeicher;

WLZ, Wärmepumpenlaufzeit.

In der Tabelle 10 ist die Jahresarbeitszahl $\left(\beta_{\mathrm{a}}\right)$ bei einem Temperaturniveau von $30^{\circ} \mathrm{C}$ auf der warmen Seite der Wärmepumpe (kPS) für die Energieabnahme der Jugendherberge dargestellt. Neben der Jahresarbeitszahl wurden die Wärmepumpenlaufzeit (WLZ), die abgegebene Wärmemenge (aWM) und die elektrische Antriebsenergie (eAE) angegeben. Der Vergleich mit Tabelle 6 zeigt, dass sich durch die Energieabnahme in der Jugendherberge die Wärmepumpenlaufzeit (WLZ) um rund $96 \mathrm{~h}$ verringert hat. Aufgrund dieser Verringerung gibt die Wärmepumpe eine um rund $769 \mathrm{kWh}$ niedrigere Wärmemenge ab und benötigt eine um rund $238 \mathrm{kWh}$ niedrigere elektrische Antriebsenergie. Die Jahresarbeitszahl ist im Vergleich zu einer vollständigen Energieabnahme um 0,01 niedriger. Anhand dieser Ergebnisse kann zusammenfassend gesagt werden, dass sich durch die verringerte Wärmepumpenlaufzeit im Sommer und Herbst die Jahresarbeitszahl geringfügig verschlechtert hat. 
Tabelle 10: Jahresarbeitszahl bezogen auf die Wärmeabnahme in der Jugendherberge

\begin{tabular}{|c|c|c|c|c|}
\hline $\mathrm{kPS}\left({ }^{\circ} \mathrm{C}\right)$ & WLZ (h) & aWM (kWh) & eAE (kWh) & $\beta_{a}$ \\
\hline 30 & 2537,90 & 19650,56 & 6336,54 & 3,10 \\
\hline$a W E, a b$ & bene Wä & emenge; & & \\
\hline $\begin{array}{l}\text { eAE, elek } \\
\text { kPS, kältt }\end{array}$ & $\begin{array}{l}\text { che Antrie } \\
\text { er Puffersp }\end{array}$ & $\begin{array}{l}\text { energie; } B_{\mathrm{a}}, \mathrm{J} \\
\text { cher; WLZ, W }\end{array}$ & $\begin{array}{l}\text { esarbeitsza } \\
\text { epumpenla }\end{array}$ & \\
\hline
\end{tabular}

\subsubsection{Optimierung bei vollständiger Wärmeabnahme}

Die Tabelle 11 zeigt die Leistungszahlen des Verdichters $\left(\varepsilon_{\mathrm{V}}\right)$ für einen optimierten Wärmepumpenbetrieb bei verschiedenen Temperaturniveaus auf der warmen Seite der Wärmepumpe (kPS). Neben den Leistungszahlen für die unterschiedlichen Jahreszeiten wurden die Verdampferleistung (VL), die elektrische Antriebsleistung des Verdichters (EAL) und die Wärmeleistung des Verdichters $(\mathrm{WL})$ dargestellt. Der Vergleich mit den Leistungszahlen $\left(\varepsilon_{\mathrm{V}}\right)$ aus Tabelle 4 zeigt, dass die Verdichterleistungen (VL) erheblich ansteigen und die elektrischen Antriebsleistungen des Verdichters (EAL) sinken. Die Wärmeleistungen $(\mathrm{WL})$ und die Leistungsziffern des Verdichters $\left(\varepsilon_{\mathrm{V}}\right)$ liegen im Vergleich zu Tabelle 4 bedeutend höher. Im Gegensatz zu Tabelle 4 ist festzustellen, dass auch im Frühling bei einem Temperaturniveau von $55^{\circ} \mathrm{C}$ auf der warmen Seite ein Betrieb der Wärmepumpe möglich ist. 
Tabelle 11: Leistungszahlen des Verdichters bei einem optimierten Wärmepumpenbetrieb für verschiedene Jahreszeiten und Temperaturniveaus

\begin{tabular}{|c|c|c|c|c|c|}
\hline Jahreszeit & $\operatorname{kPS}\left({ }^{\circ} \mathrm{C}\right)$ & $\mathrm{VL}(\mathrm{kW})$ & EAL (kW) & $\mathrm{WL}(\mathrm{kW})$ & $\varepsilon_{\mathrm{v}}$ \\
\hline Frühling & & 8,43 & 1,25 & 9,68 & 7,74 \\
\hline Sommer & 30 & 10,30 & 1,22 & 11,52 & 9,44 \\
\hline Herbst & & 10,29 & 1,22 & 11,51 & 9,43 \\
\hline Frühling & & 7,92 & 1,47 & 9,39 & 6,39 \\
\hline Sommer & 35 & 9,74 & 1,43 & 11,17 & 7,81 \\
\hline Herbst & & 9,73 & 1,43 & 11,16 & 7,80 \\
\hline Frühling & & 7,38 & 1,73 & 9,11 & 5,27 \\
\hline Sommer & 40 & 9,16 & 1,68 & 10,84 & 6,45 \\
\hline Herbst & & 9,15 & 1,68 & 10,83 & 6,45 \\
\hline Frühling & & 6,82 & 2,03 & 8,85 & 4,36 \\
\hline Sommer & 45 & 8,56 & 1,98 & 10,54 & 5,32 \\
\hline Herbst & & 8,55 & 1,98 & 10,53 & 5,32 \\
\hline Frühling & & 6,25 & 2,38 & 8,63 & 3,63 \\
\hline Sommer & 50 & 7,94 & 2,32 & 10,26 & 4,42 \\
\hline Herbst & & 7,94 & 2,32 & 10,26 & 4,42 \\
\hline Frühling & & 5,66 & 2,77 & 8,43 & 3,04 \\
\hline Sommer & 55 & 7,31 & 2,71 & 10,02 & 3,70 \\
\hline Herbst & & 7,30 & 2,71 & 10,01 & 3,69 \\
\hline
\end{tabular}

In Tabelle 12 sind die Arbeitszahlen $(\beta)$ bei verschiedenen Temperaturniveaus auf der warmen Seite der Wärmepumpe (kPS) für die unterschiedlichen Jahreszeiten dargestellt. Neben den Arbeitszahlen bei einem optimierten Wärmepumpenbetrieb und einer vollständigen Wärmeabnahme wurden die Wärmepumpenlaufzeiten (WLZ), die abgegebene Wärmemenge (aWM) und die elektrische Antriebsenergie (eAE) angegeben. Der Vergleich mit Tabelle 5 verdeutlicht, dass bei identischen Wärmepumpenlaufzeiten (WLZ) die abgegebenen Wärmemengen (aWM) erheblich steigen und die elektrischen Antriebsenergien (eAE) deutlich sinken. Es ist weiterhin festzustellen, dass auch im Frühling bei einem Temperaturniveau von $55^{\circ} \mathrm{C}$ 
auf der warmen Seite die mögliche Wärmepumpenlaufzeit realisierbar ist. Die Gegenüberstellung der Arbeitszahlen ( $\beta$ ) zeigt eine deutlich höhere Effizienz des optimierten Wärmepumpenbetriebes.

Tabelle 12: Arbeitszahlen bei einem optimierten Wärmepumpenbetrieb für verschiedene Jahreszeiten und Temperaturniveaus

\begin{tabular}{|c|c|c|c|c|c|}
\hline Jahreszeit & $\operatorname{kPS}\left({ }^{\circ} \mathrm{C}\right)$ & WLZ (h) & aWM (kWh) & eAE (kWh) & $ß$ \\
\hline Frühling & & 495,86 & 4803,22 & 1051,83 & 4,57 \\
\hline Sommer & 30 & 1619,40 & 18658,27 & 3353,34 & 5,56 \\
\hline Herbst & & 518,40 & 5968,80 & 1069,13 & 5,58 \\
\hline Frühling & & 495,86 & 4652,10 & 1157,94 & 4,02 \\
\hline Sommer & 35 & 1619,40 & 18082,78 & 3688,57 & 4,90 \\
\hline Herbst & & 518,40 & 5784,69 & 1176,46 & 4,92 \\
\hline Frühling & & 495,86 & 4514,29 & 1286,37 & 3,51 \\
\hline Sommer & 40 & 1619,40 & 17551,69 & 4096,67 & 4,28 \\
\hline Herbst & & 518,40 & 5614,79 & 1307,12 & 4,30 \\
\hline Frühling & & 495,86 & 4389,92 & 1437,11 & 3,05 \\
\hline Sommer & 45 & 1619,40 & 17064,51 & 4577,64 & 3,73 \\
\hline Herbst & & 518,40 & 5458,94 & 1461,10 & 3,74 \\
\hline Frühling & & 495,86 & 4279,01 & 1610,16 & 2,66 \\
\hline Sommer & 50 & 1619,40 & 16619,17 & 5129,87 & 3,24 \\
\hline Herbst & & 518,40 & 5316,49 & 1637,90 & 3,25 \\
\hline Frühling & & 495,86 & 4180,90 & 1805,04 & 2,32 \\
\hline Sommer & 55 & 1619,40 & 16219,36 & 5756,59 & 2,82 \\
\hline Herbst & & 518,40 & 5188,62 & 1838,53 & 2,82 \\
\hline
\end{tabular}

aWM, abgegebene Wärmemenge; $ß$, Arbeitszahl;

eAE, elektrische Antriebsenergie; kPS, kältester Pufferspeicher;

WLZ, Wärmepumpenlaufzeit.

Der Tabelle 13 kann man die Jahresarbeitszahlen $\left(\beta_{\mathrm{a}}\right)$ bei verschiedenen Temperaturniveaus auf der warmen Seite der Wärmepumpe (kPS) für die unterschiedlichen Jahreszeiten entnehmen. Neben den Jahresarbeitszahlen bei einem optimierten Wärmepumpenbetrieb und einer vollständigen Wärmeabnahme wurden die Wärmepumpenlaufzeiten (WLZ), die 
abgegebene Wärmemenge (aWM) und die elektrische Antriebsenergie (eAE) dargestellt. Aus der Gegenüberstellung mit der Tabelle 6 ist ersichtlich, dass die abgegebenen Wärmemengen (aWM) je nach Temperaturniveau auf der warmen Seite (kPS) mindestens um rund $7.950 \mathrm{kWh}$ und maximal um rund 10.566 kWh ansteigen. Der maximale Anstieg von rund $10.566 \mathrm{kWh}$ ist neben der Optimierung das Ergebnis des im Frühling realisierbaren Wärmepumpenbetriebes bei einem Temperaturniveau auf der warmen Seite von $55^{\circ} \mathrm{C}(\mathrm{kPS})$. Bei dem Vergleich der elektrischen Antriebsenergien (eAE) zeigt sich, dass im Minimum rund $1.100 \mathrm{kWh}$ und im Maximum rund $1.251 \mathrm{kWh}$ Energie eingespart werden. Die Zunahme der elektrischen Antriebsenergie um rund $758 \mathrm{kWh}$ bei einem Temperaturniveau von $55^{\circ} \mathrm{C}$ auf der warmen Seite (kPS) resultiert wiederum aus dem Ergebnis, dass im Frühling bei diesem Temperaturniveau ein Wärmepumpenbetrieb realisierbar ist. Der Vergleich mit den Jahresarbeitszahlen $\left(\beta_{\mathrm{a}}\right)$ aus Tabelle 6 zeigt, dass die Jahresarbeitszahlen bei dem niedrigsten Temperaturniveau auf der warmen Seite der Wärmepumpe (kPS) um rund 2,3 und bei einem Temperaturniveau von $55^{\circ} \mathrm{C}$ um rund 1,0 höher liegen. 
Tabelle 13: Jahresarbeitszahlen bei einem optimierten Wärmepumpenbetrieb für verschiedene Temperaturniveaus

\begin{tabular}{ccccc}
\hline kPS $\left({ }^{\circ} \mathrm{C}\right)$ & WLZ $(\mathrm{h})$ & $\mathrm{aWM}(\mathrm{kWh})$ & $\mathrm{eAE}(\mathrm{kWh})$ & $\beta_{\mathrm{a}}$ \\
\hline 30 & 2633,66 & 29430,29 & 5474,30 & 5,38 \\
\hline 35 & 2633,66 & 28519,57 & 6022,97 & 4,74 \\
\hline 40 & 2633,66 & 27680,77 & 6690,16 & 4,14 \\
\hline 45 & 2633,66 & 26913,37 & 7475,85 & 3,60 \\
\hline 50 & 2633,66 & 26214,67 & 8377,93 & 3,13 \\
\hline & & & \\
\hline & & & \\
\hline
\end{tabular}

\subsection{5 Ökonomische Betrachtung}

\subsubsection{Investitionskosten der solaren Energiegewinnung}

In Tabelle 14 sind die Investitionskosten (IK) der solaren Energiegewinnung inklusive der Mehrwertsteuer dargestellt. Der Tabelle kann entnommen werden, dass die größte Kostenposition durch die Wärmepumpentechnik und die Pufferspeicher verursacht wurde. Stellt man den gesamten Investitionskosten die Kosten für die energetische Nutzung der Dachfläche gegenüber, zeigt sich, dass diese Investitionskosten lediglich 6,36\% der gesamten Investitionssumme ausmachen. 
Tabelle 14: Investitionskosten der solaren Energiegewinnung

\begin{tabular}{lr}
\hline & \\
Konterlattung + Firstverschluss & $400,00 €$ \\
Rohrsystem + Ventilatoren & $2.500,00 €$ \\
Wärmetauscher & $870,00 €$ \\
Verrohrung Wärmetauscher (55 Meter) + Glykolgemisch & $3.250,00 €$ \\
Wärmepumpe & $17.270,00 €$ \\
1 Pufferspeicher Heizungsseite & $1.350,00 €$ \\
Installation Pufferspeicher Heizungsseite & $3.480,00 €$ \\
2 Pufferspeicher Brauchwasservorerwärmung & $6.150,00 €$ \\
Installation 2 Pufferspeicher Brauchwasservorerwärmung & $9.050,00 €$ \\
Elektroinstallation & $1.300,00 €$ \\
\hline Gesamtkosten inkl. MwSt. & $45.620,00 €$ \\
\end{tabular}

\subsubsection{Energiebezugskosten und Amortisationszeitraum bei einer vollständigen Wärmeabnahme}

Die Tabelle 15 zeigt die Energiebezugskosten bei verschiedenen Temperaturniveaus auf der warmen Seite der Wärmepumpe (kPS) für die unterschiedlichen Jahreszeiten. Neben den Energiebezugskosten bei einer vollständigen Wärmeabnahme für Strom (SKWP) und Gas (GK) wurden der Gasmengenbedarf (GMB), die Bemessungsgrundlage für die Gasabrechnung (BGGA) und die eingesparten Energiebezugskosten (eEBK) dargestellt. Aus der Tabelle ist ersichtlich, dass ab einem Temperaturniveau von $40^{\circ} \mathrm{C}$ auf der warmen Seite der Wärmepumpe die Energiebezugskosten des konventionellen Heizungssystems preiswerter sind als die Energiebezugskosten der Wärmepumpe. Der Tabelle kann weiterhin entnommen werden, dass auch bei einem niedrigeren Temperaturniveau auf der warmen Seite die Energiebezugskosten des konventionellen Heizungssystems im Frühling unter den Energiebezugskosten der Wärmepumpe liegen. Die Tabelle zeigt, dass die eingesparten Energiebezugskosten im Sommer und Herbst bei einem Temperaturniveau von $30^{\circ} \mathrm{C}$ auf der warmen Seite der Wärmepumpe höher ausfallen als bei einem Temperaturniveau von $35^{\circ} \mathrm{C}$. 
Tabelle 15: Energiebezugskosten für verschiedene Jahreszeiten und Temperaturniveaus bei einer vollständigen Wärmeabnahme

\begin{tabular}{|c|c|c|c|c|c|c|}
\hline Jahreszeit & $\operatorname{kPS}\left({ }^{\circ} \mathrm{C}\right)$ & SKWP $(€)$ & $\operatorname{GMB}\left(\mathrm{m}^{3}\right)$ & BGGA (kWh) & GK $(€)$ & eEBK $(€)$ \\
\hline Frühling & & 175,68 & 300,43 & 3424,93 & 175,36 & $-0,32$ \\
\hline Sommer & 30 & 563,72 & 1200,89 & 13690,16 & 700,94 & 137,22 \\
\hline Herbst & & 179,74 & 384,13 & 4379,05 & 224,21 & 44,47 \\
\hline Frühling & & 191,49 & 291,07 & 3318,15 & 169,89 & $-21,60$ \\
\hline Sommer & 35 & 613,76 & 1162,82 & 13256,12 & 678,71 & 64,95 \\
\hline Herbst & & 195,76 & 371,95 & 4240,23 & 217,10 & 21,34 \\
\hline Frühling & & 210,48 & 282,99 & 3226,04 & 165,17 & $-45,31$ \\
\hline Sommer & 40 & 673,98 & 1128,76 & 12867,82 & 658,83 & $-15,15$ \\
\hline Herbst & & 215,04 & 361,06 & 4116,05 & 210,74 & $-4,30$ \\
\hline Frühling & & 232,53 & 276,10 & 3147,56 & 161,16 & $-71,37$ \\
\hline Sommer & 45 & 744,39 & 1098,79 & 12526,26 & 641,34 & $-103,05$ \\
\hline Herbst & & 237,58 & 351,48 & 4006,82 & 205,15 & $-32,43$ \\
\hline Frühling & & 257,69 & 270,46 & 3083,24 & 157,86 & $-99,83$ \\
\hline Sommer & 50 & 824,98 & 1072,84 & 12230,42 & 626,20 & $-198,78$ \\
\hline Herbst & & 263,38 & 343,18 & 3912,24 & 200,31 & $-63,07$ \\
\hline Frühling & & 0,00 & 0,00 & 0,00 & 0,00 & 0,00 \\
\hline Sommer & 55 & 915,77 & 1050,95 & 11980,82 & 613,42 & $-302,35$ \\
\hline Herbst & & 292,45 & 336,18 & 3832,45 & 196,22 & $-96,23$ \\
\hline
\end{tabular}

BGGA, Bemessungsgrundlage Gasabrechnung; eEBK, eingesparte Energiebezugskosten; GK, Gaskosten; GMB, Gasmengenbedarf; kPS, kältester Pufferspeicher; SKWP, Stromkosten Wärmepumpe.

Aus der Tabelle 16 können die eingesparten Jahresenergiebezugskosten bei einem Temperaturniveau von $30^{\circ} \mathrm{C}$ auf der warmen Seite der Wärmepumpe (kPS) entnommen werden. Neben den Jahresenergiebezugskosten bei einer vollständigen Wärmeabnahme für Strom (SKWP) und Gas (GK) wurden der Gasmengenbedarf (GMB), die Bemessungsgrundlage für die Gasabrechnung (BGGA) und die eingesparten Energiebezugskosten (eEBK) dargestellt. Der Tabelle ist zu entnehmen, dass der Wärmepumpenbetrieb Jahresenergiebezugskosten in Höhe von rund $919 €$ verursacht hat. In diesem Zeitraum wären für die gleiche Wärmemenge rund $1.885 \mathrm{~m}^{3}$ Gas durch das konventionelle Heizungssystem verbraucht worden. Dieser 
Gasverbrauch hätte Kosten in Höhe von rund 1.101€ verursacht. Die jährlich eingesparten Energiebezugskosten betragen rund $181 €$. Dies entspricht einer Energiebezugskosteneinsparung von rund 16,48 \% gegenüber dem konventionellen Heizungssystem.

Tabelle 16: Eingesparte Jahresenergiebezugskosten bei einer vollständigen Wärmeabnahme

\begin{tabular}{cccccr}
\hline $\mathrm{kPS}\left({ }^{\circ} \mathrm{C}\right)$ & $\mathrm{SKWP}(€)$ & $\mathrm{GMB}\left(\mathrm{m}^{3}\right)$ & $\mathrm{BGGA}(\mathrm{kWh})$ & $\mathrm{GK}(€)$ & eEBK $(€)$ \\
\hline 30 & 919,14 & 1885,45 & 21494,14 & 1100,51 & 181,37
\end{tabular}

BGGA, Bemessungsgrundlage Gasabrechnung; eEBK, eingesparte Energiebezugskosten; GK, Gaskosten; GMB, Gasmengenbedarf; kPS, kältester Pufferspeicher; SKWP, Stromkosten Wärmepumpe.

Die Tabelle 17 zeigt den Amortisationszeitraum (AZR) der Investitionskosten (IK) bei einem Temperaturniveau von $30^{\circ} \mathrm{C}$ auf der warmen Seite der Wärmepumpe (kPS). Neben dem Amortisationszeitraum bei einer vollständigen Wärmeabnahme wurden die Investitionskosten und die jährlich eingesparten Energiebezugskosten (eEBK) dargestellt. Aus der Tabelle ist abzulesen, dass sich die Investitionskosten in einem Zeitraum von rund 252 Jahren amortisieren.

Tabelle 17: Amortisationszeitraum der Investitionskosten für die solare Energiegewinnung bei einer vollständigen Wärmeabnahme

\begin{tabular}{lcrr} 
IK $(€)$ & kPS $\left({ }^{\circ} \mathrm{C}\right)$ & eEBK $(€)$ & AZR (a) \\
\hline 45620 & 30 & 181,37 & 251,53 \\
& & \\
\hline & AZR, Amortisationszeitraum; \\
eEBK, eingesparte Energiebezugskosten; \\
IK, Investitionskosten; \\
kPS, kältester Pufferspeicher.
\end{tabular}




\subsubsection{Energiebezugskosten und Amortisationszeitraum bezogen auf die Wärmeabnahme in der Jugendherberge}

In Tabelle 18 sind die Energiebezugskosten bei einem Temperaturniveau von $30^{\circ} \mathrm{C}$ auf der warmen Seite der Wärmepumpe (kPS) für die unterschiedlichen Jahreszeiten dargestellt. Neben den Energiebezugskosten für Strom (SKWP) und Gas (GK), bezogen auf die Wärmeabnahme in der Jugendherberge, wurden der Gasmengenbedarf (GMB), die Bemessungsgrundlage für die Gasabrechnung (BGGA) und die eingesparten Energiebezugskosten (eEBK) dargestellt. Der Vergleich mit Tabelle 15 offenbart, dass sich die eingesparten Energiebezugskosten (eEBK) sowohl im Sommer als auch im Herbst aufgrund der in Tabelle 8 dargestellten geringeren Wärmeabnahme (WBD) der Jugendherberge geringfügig verringern.

Tabelle 18: Energiebezugskosten für verschiedene Jahreszeiten bezogen auf die Wärmeabnahme in der Jugendherberge

\begin{tabular}{lcrrrrr}
\hline & & & & & & \\
Jahreszeit & $\mathrm{kPS}\left({ }^{\circ} \mathrm{C}\right)$ & $\mathrm{SKWP}(€)$ & $\mathrm{GMB}\left(\mathrm{m}^{3}\right)$ & $\mathrm{BGGA}(\mathrm{kWh})$ & $\mathrm{GK}(€)$ & eEBK $(€)$ \\
\hline & & & & & & \\
Frühling & & 175,68 & 300,43 & 3424,93 & 175,36 & $-0,32$ \\
Sommer & \multirow{2}{*}{30} & 540,83 & 1152,13 & 13134,32 & 672,48 & 131,65 \\
Herbst & & 169,33 & 361,89 & 4125,55 & 211,23 & 41,90
\end{tabular}

BGGA, Bemessungsgrundlage Gasabrechnung; eEBK, eingesparte Energiebezugskosten; GK, Gaskosten; GMB, Gasmengenbedarf; kPS, kältester Pufferspeicher; SKWP, Stromkosten Wärmepumpe.

Die Tabelle 19 zeigt die eingesparten Jahresenergiebezugskosten bei einem Temperaturniveau von $30^{\circ} \mathrm{C}$ auf der warmen Seite der Wärmepumpe (kPS). Neben den Jahresenergiebezugskosten für Strom (SKWP) und Gas (GK), bezogen auf die Wärmeabnahme in der Jugendherberge, wurden der Gasmengenbedarf (GMB), die Bemessungsgrundlage für die Gasabrechnung (BGGA) und die eingesparten Energiebezugskosten (eEBK) dargestellt. Der Vergleich mit Tabelle 16 zeigt, dass sich die jährlich eingesparten Energiebezugskosten (eEBK) um rund $8 €$ reduzieren. Die Gegenüberstellung der Energiebezugskosten (eEBK) aus Tabelle 19 zeigt, dass durch den 
Wärmepumpenbetrieb eine Energiebezugskosteneinsparung von rund $16,36 \%$ gegenüber dem konventionellen Heizungssystem (GK) erzielt wurde. Damit verschlechtert sich die Energiebezugskosteneinsparung um rund $0,12 \%$ gegenüber der Einsparung bei einer vollständigen Wärmeabnahme.

Tabelle 19: Eingesparte Jahresenergiebezugskosten bezogen auf die Wärmeabnahme in der Jugendherberge

\begin{tabular}{crrrrr}
\hline $\operatorname{kPS}\left({ }^{\circ} \mathrm{C}\right)$ & $\mathrm{SKWP}(€)$ & $\mathrm{GMB}\left(\mathrm{m}^{3}\right)$ & $\mathrm{BGGA}(\mathrm{kWh})$ & GK $(€)$ & eEBK $(€)$ \\
\hline 30 & 885,84 & 1814,45 & 20684,80 & 1059,07 & 173,23
\end{tabular}

BGGA, Bemessungsgrundlage Gasabrechnung;

eEBK, eingesparte Energiebezugskosten; GK, Gaskosten;

GMB, Gasmengenbedarf; kPS, kältester Pufferspeicher;

SKWP, Stromkosten Wärmepumpe.

In der Tabelle 20 ist der Amortisationszeitraum (AZR) der Investitionskosten (IK) bei einem Temperaturniveau von $30^{\circ} \mathrm{C}$ auf der warmen Seite der Wärmepumpe (kPS) dargestellt. Neben dem Amortisationszeitraum, bezogen auf die Wärmeabnahme der Jugendherberge, wurden die Investitionskosten und die jährlich eingesparten Energiebezugskosten (eEBK) dargestellt. Aus der Tabelle ist abzulesen, dass sich der Amortisationszeitraum (AZR) im Vergleich zu Tabelle 17 um etwa 12 Jahre (Faktor 1,05) verlängert.

Tabelle 20: Amortisationszeitraum der Investitionskosten für die solare Energiegewinnung bezogen auf die Wärmeabnahme in der Jugendherberge

\begin{tabular}{cccc}
$\mathrm{IK}(€)$ & $\mathrm{kPS}\left({ }^{\circ} \mathrm{C}\right)$ & $\mathrm{eEBK}(€)$ & AZR $(\mathrm{a})$ \\
\hline 45620 & 30 & 173,23 & 263,35
\end{tabular}

AZR, Amortisationszeitraum;

eEBK, eingesparte Energiebezugskosten;

IK, Investitionskosten;

kPS, kältester Pufferspeicher. 


\subsubsection{Energiebezugskosten und Amortisationszeitraum für die Optimierung bei vollständiger Wärmeabnahme}

In Tabelle 21 sind die Energiebezugskosten bei verschiedenen Temperaturniveaus auf der warmen Seite der Wärmepumpe (kPS) für die unterschiedlichen Jahreszeiten dargestellt. Neben den Energiebezugskosten bei einem optimierten Wärmepumpenbetrieb und einer vollständigen Wärmeabnahme für Strom (SKWP) und Gas (GK) wurden der Gasmengenbedarf (GMB), die Bemessungsgrundlage für die Gasabrechnung (BGGA) und die eingesparten Energiebezugskosten (eEBK) dargestellt. Aus der Tabelle ist abzulesen, dass die Energiebezugskosten der Wärmepumpe außer im Frühling bei einem Temperaturniveau von $55^{\circ} \mathrm{C}$ auf der warmen Seite der Wärmepumpe gegenüber dem konventionellen Heizungssystem preiswerter sind. Die Tabelle zeigt, dass die eingesparten Energiebezugskosten im Frühling, Sommer und Herbst bei einem Temperaturniveau von $30^{\circ} \mathrm{C}$ auf der warmen Seite der Wärmepumpe am höchsten sind. 
Tabelle 21: Energiebezugskosten für verschiedene Jahreszeiten und Temperaturniveaus bei einer Optimierung des Wärmepumpenbetriebes bezogen auf eine vollständige Wärmeabnahme

\begin{tabular}{|c|c|c|c|c|c|c|}
\hline Jahreszeit & $\mathrm{kPS}\left({ }^{\circ} \mathrm{C}\right)$ & SKWP $(€)$ & GMB $\left(m^{3}\right)$ & BGGA (kWh) & GK $(€)$ & eEBK $(€)$ \\
\hline Frühling & & 147,05 & 443,51 & 5056,02 & 258,87 & 111,82 \\
\hline Sommer & 30 & 468,80 & 1722,83 & 19640,29 & 1005,58 & 536,78 \\
\hline Herbst & & 149,46 & 551,14 & 6282,95 & 321,69 & 172,23 \\
\hline Frühling & & 161,88 & 429,56 & 4896,95 & 250,72 & 88,84 \\
\hline Sommer & 35 & 515,66 & 1669,69 & 19034,51 & 974,57 & 458,91 \\
\hline Herbst & & 164,47 & 534,14 & 6089,15 & 311,76 & 147,29 \\
\hline Frühling & & 179,83 & 416,83 & 4751,88 & 243,30 & 63,47 \\
\hline Sommer & 40 & 572,71 & 1620,65 & 18475,46 & 945,94 & 373,23 \\
\hline Herbst & & 182,73 & 518,45 & 5910,30 & 302,61 & 119,88 \\
\hline Frühling & & 200,91 & 405,35 & 4620,97 & 236,59 & 35,68 \\
\hline Sommer & 45 & 639,95 & 1575,67 & 17962,65 & 919,69 & 279,74 \\
\hline Herbst & & 204,26 & 504,06 & 5746,26 & 294,21 & 89,95 \\
\hline Frühling & & 225,10 & 395,11 & 4504,22 & 230,62 & 5,52 \\
\hline Sommer & 50 & 717,16 & 1534,55 & 17493,86 & 895,69 & 178,53 \\
\hline Herbst & & 228,98 & 490,90 & 5596,31 & 286,53 & 57,55 \\
\hline Frühling & & 252,34 & 386,05 & 4400,95 & 225,33 & $-27,01$ \\
\hline Sommer & 55 & 804,77 & 1497,63 & 17073,01 & 874,14 & 69,37 \\
\hline Herbst & & 257,03 & 479,10 & 5461,70 & 279,64 & 22,61 \\
\hline
\end{tabular}

BGGA, Bemessungsgrundlage Gasabrechnung; eEBK, eingesparte Energiebezugskosten; GK, Gaskosten; GMB, Gasmengenbedarf; kPS, kältester Pufferspeicher; SKWP, Stromkosten Wärmepumpe.

Die Tabelle 22 zeigt die eingesparten Jahresenergiebezugskosten bei einem Temperaturniveau von $30^{\circ} \mathrm{C}$ auf der warmen Seite der Wärmepumpe (kPS). Neben den Jahresenergiebezugskosten für Strom (SKWP) und Gas (GK) bei einem optimierten Wärmepumpenbetrieb und einer vollständigen Wärmeabnahme wurden der Gasmengenbedarf (GMB), die Bemessungsgrundlage für die Gasabrechnung (BGGA) und die eingesparten Energiebezugskosten (eEBK) dargestellt. Der Vergleich mit Tabelle 16 zeigt, dass sich die jährlich eingesparten Energiebezugskosten um 639,46€ erhöhen. Die Gegenüberstellung der Energiebezugskosten aus Tabelle 22 zeigt, dass 
durch den Wärmepumpenbetrieb eine Energiebezugskosteneinsparung von rund $51,75 \%$ gegenüber dem konventionellen Heizungssystem erzielt wurde. Damit verbessert sich die Energiebezugskosteneinsparung gegenüber dem nicht optimierten Wärmepumpenbetrieb um 35,27 \%.

Tabelle 22: Eingesparte Jahresenergiebezugskosten für die Optimierung bei vollständiger Wärmeabnahme

\begin{tabular}{cccccc}
\hline $\operatorname{kPS}\left({ }^{\circ} \mathrm{C}\right)$ & $\mathrm{SKWP}(€)$ & $\mathrm{GMB}\left(\mathrm{m}^{3}\right)$ & $\mathrm{BGGA}(\mathrm{kWh})$ & GK $(€)$ & eEBK $(€)$ \\
\hline \multirow{2}{*}{30} & 765,31 & 2717,48 & 30979,26 & 1586,14 & 820,83
\end{tabular}

BGGA, Bemessungsgrundlage Gasabrechnung; eEBK, eingesparte Energiebezugskosten; GK, Gaskosten; GMB, Gasmengenbedarf; kPS, kältester Pufferspeicher; SKWP, Stromkosten Wärmepumpe.

In der Tabelle 23 ist der Amortisationszeitraum (AZR) der Investitionskosten (IK) bei einem Temperaturniveau von $30^{\circ} \mathrm{C}$ auf der warmen Seite der Wärmepumpe (kPS) dargestellt. Neben dem Amortisationszeitraum bei einem optimierten Wärmepumpenbetrieb und einer vollständigen Wärmeabnahme wurden die Investitionskosten und die jährlich eingesparten Energiebezugskosten (eEBK) dargestellt. Aus der Tabelle ist abzulesen, dass sich der Amortisationszeitraum im Vergleich zu Tabelle 17 in etwa um 196 Jahre (Faktor 4,53) verringert. 
Tabelle 23: Amortisationszeitraum der Investitionskosten für die solare Energiegewinnung bei einer Optimierung des Wärmepumpenbetriebes bezogen auf eine vollständige Wärmeabnahme

\begin{tabular}{cccc}
$\mathbb{I K}(€)$ & $\mathrm{kPS}\left({ }^{\circ} \mathrm{C}\right)$ & $\mathrm{eEBK}(€)$ & $\mathrm{AZR}(\mathrm{a})$ \\
\hline 45620 & 30 & 820,83 & 55,58
\end{tabular}

AZR, Amortisationszeitraum;

eEBK, eingesparte Energiebezugskosten; IK, Investitionskosten;

kPS, kältester Pufferspeicher. 


\section{$5 \quad$ Diskussion}

\subsection{Solardach-Luft-Kollektor}

Es wird darauf hingewiesen, dass die dargestellten einzelnen Tage das Ergebnis einer getroffenen Auswahl innerhalb der entsprechenden Jahreszeit sind und dem Aufzeigen von Tendenzen dienen. Die Messwertabstände zwischen den dargestellten Tagen sind aus diesem Grund nicht eindeutig zu interpretieren.

\subsubsection{Globalstrahlungsverlauf in Kollektorebene}

Die in Abbildung 13 dargestellten Globalstrahlungsverläufe entsprechen dem in der Literatur beschriebenen typischen Glockenkurvenverlauf der Globalstrahlung $[34,38,56]$. Die Beobachtung der steileren Anstiege der Globalstrahlungsverläufe in der ersten Tageshälfte gegenüber der zweiten Tageshälfte ist durch die um $25^{\circ}$ angestellte Kollektorfläche und die sich daraus ergebenden günstigeren Sonneneinstrahlungswinkel in der ersten Tageshälfte zu erklären [38,40]. Der beobachtete flachere Anstieg der Globalstrahlungsverläufe bei Sonnenaufgang und Sonnenuntergang gegenüber dem restlichen Tagesgang ist anhand des Sonnenverlaufes herzuleiten [40]. Die Sonne geht auf der Seite der genutzten Dachfläche auf und das Solarimeter erfasst nach relativ kurzer Zeit die ersten direkten Sonnenstrahlen. Bei Sonnenuntergang verlängert sich der Zeitraum zwischen den letzten erfassten direkten Sonnenstrahlen und dem tatsächlichen Sonnenuntergang am Horizont aufgrund des Kollektoranstellungswinkels. Die flacheren Globalstrahlungsverläufe bei Sonnenaufgang und Sonnenuntergang lassen sich somit als Zeitraum zwischen Sonnenaufgang und Sonnenuntergang und der durch das Solarimeter erfassten direkten Sonnenstrahlung in Kollektorebene beschreiben. Als Ursache für die Ausreißer bei der Globalstrahlungskurve des Sommertages werden Reflektionen vermutet. Eine mögliche Begründung ist die Reflektion der Strahlung durch eine 
vereinzelte vorbeiziehende weiße Schleierwolke. Eine weitere mögliche Erklärung ist die Reflektion der Strahlung durch die Wasseroberfläche des Meeres [68]. Da diese Ausreißer sowohl bei der Globalstrahlung als auch bei der Lufttemperaturerhöhung wiederzufinden sind, kann eine fehlerhafte Messung an dieser Stelle ausgeschlossen werden. Die genaue Ursache der Ausreißer kann im Nachhinein nicht eindeutig bestimmt werden. Aufgrund der getroffenen Auswahl innerhalb der Jahreszeit sind die absoluten Höhen der Globalstrahlungswerte nicht eindeutig interpretierbar. Deshalb ist es möglich, dass bei einer anderen Auswahl die Messwerte gegenüber den dargestellten abweichen können.

\subsubsection{Temperaturerhöhung und Betriebsverhalten}

Der Vergleich der Abbildung 13 mit Abbildung 14 hat gezeigt, dass die Temperaturdifferenz für den Sommertag trotz gleicher Globalstrahlungsintensität in der zweiten Tageshälfte deutlich höher als für den Frühlingstag ist. Bei der Betrachtung der mittleren Umgebungstemperatur zwischen Sonnenaufgang und Sonnenuntergang zeigt sich, dass die Lufttemperatur des Frühlingstages mit $10^{\circ} \mathrm{C}$ deutlich unter der Lufttemperatur des Sommertages mit $22{ }^{\circ} \mathrm{C}$ liegt. Die niedrigen Temperaturdifferenzen des Frühlingstages lassen sich somit durch Wärmeverluste, die durch Konvektion und Wärmestrahlung verursacht wurden, erklären. Die bei diesen beiden Tagen aufgetretenen Unregelmäßigkeiten im Bereich der maximalen Temperaturdifferenzen lassen ebenfalls Wärmeverluste vermuten. Die Betrachtungen der Windgeschwindigkeiten im Tagesgang haben keine auffälligen Unterschiede ergeben. Die minimalen und maximalen Windgeschwindigkeiten schwanken relativ gleichbleibend im Tagesverlauf. Der Schwankungsbereich liegt für den Sommertag zwischen $1 \mathrm{~m} / \mathrm{s}$ und $5 \mathrm{~m} / \mathrm{s}$ und für den Frühlingstag zwischen $1 \mathrm{~m} / \mathrm{s}$ und $6 \mathrm{~m} / \mathrm{s}$. Anhand der schwankenden Windgeschwindigkeiten lässt sich vermuten, dass dieser Einfluss über den gesamten Tagesverlauf gleichbleibend ist. Die Unregelmäßigkeiten sind jedoch nur bei hohen Temperaturdifferenzen deutlich ausgeprägt. Ein 
denkbarer Erklärungsansatz ist, dass die wechselhaften Windgeschwindigkeiten bei höheren Temperaturdifferenzen größere Konvektionsverluste hervorrufen als bei niedrigeren Temperaturdifferenzen. Die Literatur bestätigt eine Einflussnahme der Windgeschwindigkeiten auf die erzielbare Temperaturdifferenz bei nicht abgedeckten Absorbern. Untersuchungen, die den Erklärungsansatz bestätigen, sind jedoch nicht bekannt $[25,34,37,40,56]$.

Die in Abbildung 14 beschriebenen höheren Temperaturdifferenzen in der zweiten Tageshälfte werden durch Abbildung 15 untermauert und deuten auf eine Pufferwirkung des Solardach-Luft-Kollektors hin. Die in Abbildung 16 dargestellten linearen Regressionsgeraden zeigen den Temperaturverlauf der ersten und zweiten Tageshälfte für ausgewählte Jahreszeiten in Abhängigkeit von der Globalstrahlung. Auffällig ist, dass die Regressionsgeraden des Sommertages sich nicht wie die übrigen Geraden mit Zunahme der Globalstrahlungsintensität annähern. Dies liegt darin begründet, dass bei dem dargestellten Sommertag die Temperaturdifferenzspreizung im Vergleich zu Abbildung 15 bereits mit Abnahme der Globalstrahlung deutlich ausgeprägter ist. Die Begründung für die höhere Temperaturdifferenz liegt wiederum in der vermuteten Pufferwirkung, die in den Sommermonaten aufgrund der höheren erzielbaren Temperaturen deutlich ausgeprägter zu sein scheint.

In Abbildung 17 sind die Temperaturdifferenzen in Abhängigkeit von der Zeit dargestellt. Durch die Spiegelung der Temperaturdifferenzen der ersten Tageshälfte sind die höheren Temperaturdifferenzen in der zweiten Tageshälfte veranschaulicht worden. Es ist jedoch davon auszugehen, dass sich die vermutete Pufferwirkung des Solardach-Luft-Kollektors bereits in der ersten Tageshälfte aufbaut. Die gebildete Differenz aus der Temperaturdifferenz in der zweiten Tageshälfte und der gespiegelten ersten Tageshälfte kann daher nur eine Tendenz aufzeigen und ist nicht weiter quantifizierbar. Es wird weiterhin vermutet, dass zwischen der Pufferwirkung und der Dachraumtemperatur ein Zusammenhang besteht. Es ist denkbar, dass sich durch die geringe Luftgeschwindigkeit der Kollektorboden mit Zunahme der 
Globalstrahlung aufheizt und durch Wärmeleitung, Wärmestrahlung und Konvektion die Dachraumtemperatur erhöht. Mit Abnahme der Globalstrahlung kehrt sich dieses Verhältnis um und die Dachraumluft erwärmt den Kollektorboden, der die übertragene Wärme an die Kollektorluft abgibt. Die in Abbildung 17 und Abbildung 18 dargestellte Temperaturdifferenz zwischen Dachraum- und Umgebungstemperatur stützt diese Annahme. Um eine exakte Aussage über die Pufferwirkung des Solardaches treffen zu können, muss anhand der Temperaturen der Kollektorbauteile der genaue Zeitraum und die Pufferwirkung bestimmt werden. Da bei diesem Versuchsaufbau keine Temperaturmessungen an den Bauteilen des Solardach-Luft-Kollektors vorgesehen waren, ist dieser Zeitpunkt und die Höhe der Pufferwirkung nicht genauer quantifizierbar. Es ist jedoch davon auszugehen, dass sich dieser Effekt bei einem reinen Bedarfsbetrieb des Solardaches durch die ruhende Luft im Kollektor an sonnigen Tagen deutlich verstärkt.

Die Annahme einer Pufferwirkung bietet ebenfalls einen Erklärungsansatz für die aufgetretenen positiven Temperaturdifferenzen nach Sonnenuntergang. Eine mögliche Erklärung ist, dass in bewölkten Nächten durch geringere Wärmeverluste die Pufferwirkung des Solardach-Luft-Kollektors ausreicht, um eine geringfügige Temperaturerhöhung bis in die Morgenstunden zu realisieren. Eine im Nachhinein gesicherte Aussage über den Bewölkungsgrad in der Nacht ist auf der Grundlage der aufgezeichneten Daten nicht möglich. Für eine Aussage über den Bewölkungsgrad muss die Himmelstemperatur bestimmt werden. Der Bewölkungsgrad kann anhand des Vergleiches der Himmelstemperatur mit der Umgebungstemperatur ermittelt werden $[3,12,34]$.

Anhand der Verläufe der Temperaturdifferenz und der Globalstrahlung in Kollektorebene wurde in Abbildung 19 die Reaktionszeit des Solardach-LuftKollektors hergeleitet. Auffällig ist, dass der Temperaturverlauf trotz der vermuteten Pufferwirkung relativ unmittelbar dem Globalstrahlungsverlauf folgt. Die schnelle Reaktionszeit des Solardach-Luft-Kollektors ist zum einen auf die gute Wärmeleitfähigkeit des Absorbers zurückzuführen, zum anderen ermöglicht die geringe spezifische Wärmekapazität der Luft eine schnelle 
Lufttemperaturerhöhung [23, 32]. Durch diese physikalischen Eigenschaften ist es möglich, bereits kurze Sonnenscheinphasen zur Energiegewinnung zu nutzen (vgl. Abb. 21).

Der in der Literatur beschriebene lineare Zusammenhang zwischen der Globalstrahlungsintensität und der Temperaturerhöhung bestätigt die Ergebnisse aus Abbildung 16 [34, 56]. Die von Grimm (1984) beschriebene negative Temperaturdifferenz nach Sonnenaufgang bei Globalstrahlungswerten unter $50 \mathrm{~W} / \mathrm{m}^{2}$ wird durch die eigenen Untersuchungen untermauert [34]. Die einschlägige Fachliteratur weist darauf hin, dass bei Kollektoren mit größerer Eigenmasse ein geringfügiges Nachlaufen der Kollektoraustrittsluft vorhanden sein kann, geht jedoch nicht explizit auf eine Pufferwirkung in der zweiten Tageshälfte ein [76]. Dies kann zum einen den Grund haben, dass die in der Literatur beschriebenen Versuche mit deutlich höheren Luftgeschwindigkeiten durchgeführt wurden. Zum anderen wurde bei den Untersuchungen an Freilandprüfständen lediglich eine freistehende Dachhälfte untersucht. Hinzu kommt, dass bei einigen Untersuchungen der Kollektorboden zusätzlich isoliert wurde. Die erzielten Temperaturdifferenzen liegen im Vergleich mit den in der Literatur angegebenen Daten im für SolarLuft-Kollektoren typischen Bereich. Grimm (1984) erläutert in seinen Untersuchungen, dass sich mit zunehmender Kollektorlänge die Lufttemperatur der Absorbertemperatur annähert und die Wärmeverluste mit der Kollektorlänge überproportional zunehmen. In seinen Untersuchungen konnte Grimm (1984) nachweisen, dass dieses Verhältnis bei höheren Strömungsgeschwindigkeiten weniger ausgeprägt ist [34]. Anhand dieser Erkenntnisse ist davon auszugehen, dass sich durch die geringe Luftgeschwindigkeit und bei einer Kollektorlänge von $6 \mathrm{~m}$ der von Grimm (1984) beschriebene überproportionale Wärmeverlust deutlich verstärkt. Aus der Literatur konnte entnommen werden, dass mit Zunahme der Luftgeschwindigkeit die Temperaturerhöhung sinkt [34,56]. Grimm (1984) und Lücke (1984) weisen in ihren Untersuchungen darauf hin, dass durch eine Erhöhung der Luftgeschwindigkeit und durch Verwirbelungseinbauten im Strömungskanal eine höhere Turbulenz des Luftstromes den Wärmeabtrag 
vom Absorber verbessern $[34,56]$. Es bleibt jedoch zu bedenken, dass durch diese Maßnahmen der statische Druck des Kollektors zunimmt und damit die benötigte Ventilatorantriebsenergie ansteigt. Aus der Literatur wird deutlich, dass sich der grundlegende Zusammenhang zwischen der Temperaturerhöhung und der Globalstrahlungsintensität bei den verglichenen Solar-LuftKollektoren widerspiegelt. Ein direkter Vergleich ist jedoch aufgrund der unterschiedlichen Kollektorbauformen und Umweltbedingungen nicht möglich.

\subsubsection{Kollektorwirkungsgrad}

Der in der Literatur angegebene Wirkungsgradverlauf in Abhängigkeit von der Zeit unterscheidet sich deutlich von dem in Abbildung 20 dargestellten Verlauf. Grimm (1984) beschreibt anhand seiner Untersuchungen den charakteristischen Wirkungsgradverlauf wie folgt: „Nach einer Anlaufphase, die etwa ein bis zwei Stunden andauert und durch einen ansteigenden Kollektorwirkungsgrad gekennzeichnet ist, verläuft der Kollektorwirkungsgrad für die folgenden 6 bis 8 Stunden nahezu konstant. [ ...] Gegen Abend nimmt der Kollektorwirkungsgrad mit sinkender Strahlungsintensität über einen Zeitraum von ein bis zwei Stunden erheblich ab" [34]. Die in Abbildung 20 dargestellten Wirkungsgradverläufe dagegen steigen zwar mit Zunahme der Globalstrahlungsintensität (Abb. 13) an, schwanken jedoch zum Teil erheblich im Tagesgang und nehmen mit Abnahme der Globalstrahlungsintensität deutlich zu. Die Schwankungen innerhalb des Tagesganges lassen sich auf die in Abbildung 14 dargestellten Schwankungen bei den Temperaturdifferenzen zurückführen. Der Vergleich mit den Temperaturdifferenzverläufen zeigt, dass die charakteristischen Verläufe sich in den Wirkungsgradverläufen annähernd widerspiegeln. Mit Hilfe der Formel (4) zur Wirkungsgradberechnung und der zuvor erläuterten höheren Temperaturdifferenz in der zweiten Tageshälfte lässt sich die Ursache für den unterschiedlichen Wirkungsgradverlauf herleiten. Durch die erzielten höheren Temperaturdifferenzen in der zweiten Tageshälfte erhöht sich die Nutz- 
leistung des Kollektors bei einer gleichzeitigen Verringerung der in der Kollektorebene eingestrahlten Globalstrahlung. Anhand dieses verschobenen Verhältnisses ist der unterschiedliche Wirkungsgradverlauf zu erklären. Nach der Definition ist der Wirkungsgrad das Verhältnis zwischen der abgegebenen Nutzleistung und der in Kollektorebene einstrahlenden Globalstrahlung [35]. Da die aufgetretenen höheren Temperaturdifferenzen in der zweiten Tageshälfte auf keinen logischen Zusammenhang mit der Globalstrahlungsintensität zurückzuführen sind, ist für diesen Zeitraum die Wirkungsgradberechnung zu verwerfen. Die in Abbildung 22 dargestellten Wirkungsgradverläufe der ersten Tageshälfte liegen erheblich niedriger als die in der Literatur beschriebenen Wirkungsgradverläufe $[34,42,56]$. Die Ursache für die sehr niedrigen Wirkungsgrade ist die in Abbildung 25 dargestellte geringe Luftgeschwindigkeit im Kollektor. Zum einen ist der geförderte Massenstrom erheblich geringer als bei höheren Luftgeschwindigkeiten und zum anderen ist zu vermuten, dass sich der bereits bei der Temperaturerhöhung beschriebene überproportionale Wärmeverlust zusätzlich negativ auf den Wirkungsgrad auswirkt. Die Betrachtung der Formel (4) zur Wirkungsgradberechnung verdeutlicht, dass durch die Erhöhung der Luftgeschwindigkeit der Massenstrom steigt und trotz einer zu erwartenden geringeren Temperaturdifferenz eine deutliche Wirkungsgraderhöhung zu erzielen ist [34, 56].

\subsubsection{Luftabsaugung}

Der in Abbildung 23 dargestellte geförderte Luftvolumenstrom in Abhängigkeit der Kollektorfelder hat gezeigt, dass durch das Schließen einzelner Kollektorfelder der Luftvolumenstrom abnimmt und die Luftvolumenstromschwankungen innerhalb einer Versuchsgruppe relativ gering sind. Es konnte weiterhin gezeigt werden, dass sich erst mit nur einem geöffneten Kollektorfeld der Luftvolumenstrom erheblich verringert. Die Begründung für diese geringen Abweichungen ist anhand der Ergebnisse aus Abbildung 24 herzuleiten. Aus den Ergebnissen geht hervor, dass bei 
einem gleichzeitigen Betrieb der fünf Kollektorfelder kein Einfluss von den Kollektorfeldern auf den geförderten Luftvolumenstrom ausgeht. Es wurde nachgewiesen, dass erst nach der Abtrennung des Wärmetauschers eine Luftvolumenstromzunahme realisiert werden kann. Die Ursache für die in Abbildung 24 dargestellten Ergebnisse ist im verwendeten Rohrsystem zur Luftabsaugung zu suchen. Der in Abbildung 5 dargestellten schematischen Luftabsaugung kann entnommen werden, dass die zwei Rohre vor dem Wärmetauscher auf ein Rohr hinter dem Wärmetauscher reduziert worden sind und somit der Rohrquerschnitt halbiert wurde. Das Ergebnis aus Abbildung 24 zeigt, dass auch nach dem Abtrennen dieses Rohres keine Luftvolumenstromzunahme eingetreten ist. Dies liegt darin begründet, dass der an dem Wärmetauscher angebrachte Trichter nicht entfernt werden konnte und die Rohraufnahmeöffnung dem Rohrquerschnitt nach dem Wärmetauscher entspricht. Es wird vermutet, dass der geringfügig niedrigere Luftvolumenstrom durch Verwirbelungen an der Rohraufnahmeöffnung entstanden ist. Durch die Ergebnisse könnte geschlussfolgert werden, dass durch das Schließen einzelner Kollektorfelder der Einfluss der Kollektorfelder auf das geförderte Luftvolumen zunimmt. Diese Einflusszunahme wäre durch das entstehende günstigere Verhältnis der hydraulischen Durchmesser zwischen dem Kollektor und dem Rohrleitungssystem begründbar. Auffällig ist jedoch, dass der erwartete negative Einfluss des Insektenschutzgitters auf den Strömungswiderstand durch die Untersuchungen mit nur einem geöffneten Kollektorfeld nicht nachgewiesen werden konnte. Der Grund der Luftvolumenstromabnahme wird deshalb in den durch die Luftsammeltrichter verursachten Querschnittsverengungen vermutet. Um eine gesicherte Aussage über diese Annahme treffen zu können, wäre es notwendig gewesen, das Insektenschutzgitter auf der gesamten Lufteintrittsfläche eines Kollektorfeldes zu entfernen. Dies war jedoch aufgrund der baulichen Gegebenheiten nicht möglich und sollte Bestandteil weiterer Untersuchungen werden.

Die in Abbildung 25 und Abbildung 26 dargestellten Luftgeschwindigkeiten für die jeweiligen Anlagenabschnitte zeigen, dass die Luftgeschwindigkeit in den Rohren zur Luftabsaugung um ein Vielfaches höher ist als die Luft- 
geschwindigkeit im Kollektor. Es wurde gezeigt, dass sich durch die Halbierung des Rohrquerschnittes nach dem Wärmetauscher die Luftgeschwindigkeit im Vergleich zu den beiden Rohren vor dem Wärmetauscher verdoppelt. Bei den Untersuchungen mit nur einem betriebenen Kollektorfeld wurde erwartet, dass sich die Luftgeschwindigkeit in dem Rohr nach dem Wärmetauscher erheblich verringert. Die Ergebnisse der Abbildung 26 haben jedoch gezeigt, dass sich die Luftgeschwindigkeit zwar verringert hat, das Niveau aber immer noch höher als erwartet war. Eine durch Querschnittsverengungen verursachte Zunahme der Luftgeschwindigkeit wurde bei der Berechnung der Luftgeschwindigkeit in den Strömungskanälen nicht berücksichtigt.

Die Literaturangaben beziehen sich hauptsächlich auf den Druckabfall und den Energiebedarf für die Luftströmung in Solar-Luft-Kollektoren [34, 42, 56]. Grimm (1984) hat jedoch in seinen Untersuchungen bereits darauf hingewiesen, dass der verwendete Luftkanal vom Solar-Luft-Kollektor bis zum Ventilator einen möglichst großen Querschnitt haben muss, damit der Strömungswiderstand möglichst gering ist. Für einen möglichst geringen Strömungswiderstand gibt Grimm (1984) eine Luftgeschwindigkeit von $5 \mathrm{~m} / \mathrm{s}$ im Luftkanal an. Der anteilige Energieaufwand beträgt bei einer Luftgeschwindigkeit von $3 \mathrm{~m} / \mathrm{s}$ je nach Kollektorbauform zwischen 6 bis $11 \%$ der Kollektornutzenergie [34]. Für den getesteten Solardach-Luft-Kollektor liegt der anteilige Energieaufwand bei einer Luftgeschwindigkeit von $0,2 \mathrm{~m} / \mathrm{s}$ dagegen bei $7,62 \%$ der Nutzenergie. Dieser prozentuale Anteil ist im Verhältnis zu den in der Literatur angegebenen Anteilen höher als erwartet. Bei den Angaben der Literatur ist anzumerken, dass der zusätzliche Strömungswiderstand des Wärmetauschers in den Berechnungen nicht enthalten ist [34]. Die eigenen Untersuchungen haben gezeigt, dass bei der Auslegung des Rohrleitungssystems zur Luftabsaugung eine Optimierungsmöglichkeit besteht. Aus diesem Grund werden in den nachfolgenden Ausführungen Möglichkeiten einer Optimierung des Rohrleitungssystems zur Luftabsaugung diskutiert. Für die Optimierungsmöglichkeiten kann auf die Erkenntnisse aus der Lüftungstechnik zurückgegriffen werden. Im Folgenden 
werden zwei unterschiedliche Ansätze zur Dimensionierung von Rohrsystemen erörtert.

Der erste Ansatz basiert auf der computergestützten Berechnung eines konstanten statischen Druckes im gesamten Rohrsystem. Ziel dieses Ansatzes ist es, durch unterschiedliche Rohrdurchmesser einen Druckabfall im Rohrsystem zu vermeiden. Hierdurch wird erreicht, dass an allen Luftentnahmestellen die gleiche Luftmenge ohne Drosselung gefördert wird [54].

Auf einer vorgegebenen Luftgeschwindigkeit im Rohrsystem basiert der zweite Ansatz. Der benötigte Rohrdurchmesser wird aus der vorgegebenen Luftgeschwindigkeit und dem geförderten Luftvolumenstrom errechnet [26]. Die Regulierung des Luftvolumenstromes in den einzelnen Feldern kann durch einen Volumenstromregler oder mechanisch erfolgen. Der Luftvolumenstromregler wird auf eine bestimmte Luftgeschwindigkeit eingestellt und regelt durch Luftgeschwindigkeitsmessungen die Luftmenge selbstständig. Da es das Ziel ist, den Anlagenwiderstand zu reduzieren, und der Volumenstromregler zur Regulierung eine zusätzliche Druckerhöhung von mindestens 30 - 50 Pa benötigt, scheidet dieser als Regulierungseinrichtung aus [63]. Unter Zuhilfenahme einer Irisblende ist eine rein mechanische Regulierung des Volumenstromes möglich. Die Irisblenden werden für jedes einzelne Kollektorfeld durch einen hydraulischen Abgleich auf die vorgegebene Luftgeschwindigkeit eingestellt und gewährleisten dadurch eine gleichmäßige Luftmengenförderung. [28,63]. Für die Praxis erscheint der zweite Ansatz zur Dimensionierung des Rohrleitungssystems am praktikabelsten. Diese Dimensionierung benötigt keine aufwändigen und teuren Rechenprogramme und ist mit einfachen Mitteln realisierbar. Für die Bestimmung der Auslegungsluftgeschwindigkeit des Rohrsystems sind folgende Zusammenhänge zu beachten: Mit zunehmender Luftgeschwindigkeit steigt der Strömungswiderstand im Rohrsystem im Quadrat zur Luftgeschwindigkeit und erhöht dadurch den Energieaufwand für den Lufttransport. Aus wirtschaftlichen Gesichtspunkten ist nach Literaturangaben eine maximale Luftgeschwindigkeit in Lüftungsanlagen von $5 \mathrm{~m} / \mathrm{s}$ 
nicht zu überschreiten. Die in der Literatur empfohlene Luftgeschwindigkeit in Luftkanälen mit Luftvolumenströmen bis zu $2.000 \mathrm{~m}^{3} / \mathrm{h}$ liegt zwischen $1-4 \mathrm{~m} / \mathrm{s}$. Je geringer die Luftgeschwindigkeit ist, desto niedriger ist der Druckverlust und desto geringer ist der Energieaufwand für den Lufttransport. Geringe Luftgeschwindigkeiten haben zur Folge, dass große Kanalquerschnitte erforderlich sind, die die Investitionskosten erhöhen $[24,26,28,32,67]$. Die Auswahl der Auslegungsluftgeschwindigkeit sollte demnach zwischen dem eingesparten Energieaufwand für den Lufttransport und den zu tätigenden Investitionskosten abgewogen werden. Neben der richtigen Auswahl der Luftgeschwindigkeit verringern möglichst kurze Rohrleitungen und das Vermeiden von scharfen Ein- und Ausströmkanten den Energieaufwand für den Luftransport [28,67]. Aus diesen Erkenntnissen lässt sich anhand der in Abbildung 25 dargestellten Luftgeschwindigkeiten ableiten, dass der Rohrdurchmesser für die Luftabsaugung bei einer Luftgeschwindigkeit von $5 \mathrm{~m} / \mathrm{s}$ um circa das Dreifache nach dem Wärmetauscher vergrößert werden müsste, wenn der geförderte Luftvolumenstrom beibehalten wird. Weiterhin scheint es aus strömungstechnischer Sicht sinnvoller zu sein, auf die Luftsammeltrichter zu verzichten und die Kollektorluft direkt durch eine zu ermittelnde Anzahl von Rohranschlüssen abzusaugen. Ebenfalls strömungstechnisch ineffizient sind die verwendeten flexiblen Schläuche, die aufgrund ihrer inhomogenen Oberfläche einen hohen Druckverlust verursachen. Eine Reduzierung des Strömungswiderstandes des Wärmetauschers ist bei gleichbleibender Kanalquerschnittsfläche durch einen schrägen Einbau möglich. Die dadurch verursachte Querschnittsvergrößerung der luftdurchströmten Fläche reduziert den Druckverlust am Wärmetauscher. Weiterhin ist abzuwägen, ob es sinnvoll ist, den Wärmetauscher mit einem leicht zu reinigenden Filter zum Schutz vor Verschmutzungen zu versehen [28, 32, 61, 67]. Bei der Planung ist ebenfalls der von den Ventilatoren zu überwindende Strömungswiderstand des Wetterschutzgitters zu beachten.

Neben den Druckverlusten hat die richtige Auswahl der eingesetzten Ventilatoren einen entscheidenden Einfluss auf den Energieaufwand für den 
Lufttransport. Die Ventilatorenantriebe werden in Wirkungsgradklassen unterteilt; je nach verwendeten Elektromotoren unterscheiden sich diese Wirkungsgrade zum Teil erheblich. Ausschlaggebend ist neben einem möglichst hohen Wirkungsgrad die richtige Auswahl der Baugröße. Die Auswahl der Baugröße ist so zu treffen, dass der Arbeitspunkt des Ventilators im Bereich des maximalen Wirkungsgrades liegt [24, 67].

\subsection{Berechnungen}

\subsubsection{Energetische Betrachtung des Daches}

Bei der Betrachtung der thermischen Energien ist zu erwähnen, dass diese aufgrund von unterschiedlichen Witterungsbedingungen von Jahr zu Jahr Schwankungen unterliegen können. Es ist weiterhin zu erwähnen, dass es durch eine Mittelwertbildung für fehlende Tage zu geringfügigen Abweichungen gekommen sein kann. Für die Mittelwertbildung wurde unterstellt, dass bei fehlenden Tagen diese Tage dem Mittelwert der übrigen Tage in einem Monat entsprechen.

Die in der Abbildung 27 dargestellte thermische Nutzenergie gibt die maximal mögliche Wärmeentzugsmenge durch die Wärmepumpe bei einer Abkühlung der Luft auf $10^{\circ} \mathrm{C}$ an. Der Abbildung ist zu entnehmen, dass es in den Monaten Dezember, Januar und Februar nicht möglich ist, einen Nutzenergiegewinn zu erzielen. Der Grund dafür ist, dass die Lufttemperaturen in diesen Monaten permanent unter $10^{\circ} \mathrm{C}$ liegen. Die eingesetzte Wärmepumpe ist zwar in der Lage, latente Wärme der Luft bis zu einer Temperatur von $-10^{\circ} \mathrm{C} z u$ entziehen, kann diese niedrigen Lufttemperaturen aufgrund der Auslegung des Wärmetauschers jedoch nicht nutzen [65]. Es bleibt zu bedenken, dass durch ein sinkendes Temperaturniveau der Wärmequelle die Arbeitszahl der Wärmepumpe ebenfalls sinkt. Für die Anhebung der Arbeitszahl muss es aus technischer Sicht daher das Ziel sein, die Lufttemperatur des Solardach-Luft-Kollektors besonders im Frühling und Herbst möglichst dicht an die Absorbertemperatur heranzuführen. Durch die dadurch zu 
realisierenden höheren Soletemperaturen muss der Verdichter eine kleinere Druckdifferenz überwinden und benötigt demzufolge, bezogen auf die Verdampferleistung, eine geringere Elektroantriebsleistung bei einer höheren Wärmeentzugsleistung [47]. Wird eine größere Wärmeentzugsleistung benötigt, so ist diese durch eine Ausdehnung der Kollektorfläche zu erzielen. Dies trifft jedoch nur zu, wenn unterstellt wird, dass der Aufbau des verwendeten Solardach-Luft-Kollektors und die Luftgeschwindigkeit von 0,2 m/s bereits das aus technischer und ökonomischer Sicht zu erzielende Optimum für einen möglichst effizienten Wärmepumpenbetrieb darstellt. Der Aussage liegen folgende Überlegungen zugrunde: Die Umgebungsluft besitzt im Verhältnis ein höheres thermisches Energieangebot als die durch den Solardach-Luft-Kollektor erzielte Temperaturdifferenz. Durch eine Erhöhung des Luftvolumenstromes ist es demnach möglich, eine größere Wärmemenge der Luft zu entziehen. Durch die Erhöhung des Luftvolumenstromes steigen jedoch auch die Luftgeschwindigkeiten im System. Die steigende Luftgeschwindigkeit im Kollektor hat zur Folge, dass die Lufttemperaturerhöhung sinkt $[34,56]$ und somit niedrigere Soletemperaturen erzielt werden. Durch den im Quadrat zur Luftgeschwindigkeit steigenden Strömungswiderstand erhöht sich ebenfalls die benötigte elektrische Antriebsenergie der Ventilatoren. Anhand dieser Überlegungen ist die Erhöhung des Luftvolumenstromes bei gleichbleibender Kollektorfläche kontraproduktiv.

Aus der Literatur sind keine direkten Aussagen darüber zu entnehmen, mit welcher Luftgeschwindigkeit die größte Annäherung an die Absorbertemperatur zu erzielen ist $[34,56]$. Die Empfehlungen aus der Literatur beziehen sich vornehmlich auf zusätzliche Verwirbelungseinbauten, die durch eine verbesserte turbulente Luftströmung den Wärmeabtrag vom Absorber zwar verbessern, aber im Gegenzug auch eine höhere zu überwindende Gesamtdruckdifferenz verursachen [34,56]. Grimm (1984) empfiehlt anhand seiner Untersuchungen für die Brauchwasservorerwärmung mit einer Luft/Wasser Wärmepumpe Luftgeschwindigkeiten zwischen 1,5 und $3,0 \mathrm{~m} / \mathrm{s}$ mit dem zusätzlichen Einbau von Verwirbelungsstegen [34]. Die bei den Untersuchungen von Lücke (1984) 
verwendeten Luftgeschwindigkeiten von 1,2 und 2,4 m/s haben sich als resultierende Größe aus dem benötigten Luftvolumen der Wärmepumpe und der zur Verfügung stehenden Fläche des Freilandprüfstandes ergeben [56]. Die von Grimm (1984) getroffene Aussage, dass höhere Temperaturdifferenzen als 10 bis $15 \mathrm{~K}$ aufgrund einer möglichen Überhitzung des Verdichters zu vermeiden sind, ist aufgrund der in den Jahren stattgefundenen Weiterentwicklung der Wärmepumpentechnik nicht mehr gültig [34]. Die Verdichter der heutigen Wärmepumpen sind deutlich leistungsfähiger und besitzen als Überhitzungsschutz ein eingebautes MOPExpansionsventil. Dieses MOP-Expansionsventil reguliert durch die Anpassung des Solevolumenstromes die, besonders in den Sommermonaten auftretenden, hohen Soletemperaturen und verhindert so ein Überhitzen des Verdichters [65]. In weiterführenden Versuchen ist zunächst die Frage zu klären, mit welcher Luftgeschwindigkeit die größtmögliche Temperaturdifferenz zwischen der Kollektoreintritts- und Kollektoraustrittsluft erzielt werden kann. Es gilt weiterhin zu klären, ab welcher Luftgeschwindigkeit, bezogen auf die jeweilige Kollektorlänge, keine nennenswerte Temperaturerhöhung aufgrund der Zunahme von Wärmeverlusten erzielt werden kann.

Bei der Betrachtung der bereinigten Energie der Umgebungsluft wurde die Ventilatorantriebsenergie des Kollektorbetriebes aufgrund der bei der Luftabsaugung diskutierten Gegebenheiten angenommen. Es wird jedoch erwartet, dass die Ventilatorantriebsenergie bei dem Ansaugen von Umgebungsluft über einen Bypass deutlich unter der des Kollektorbetriebes liegt. Die Voraussetzung für diese Annahme ist allerdings die bereits bei der Luftabsaugung diskutierte Optimierung des Rohrleitungssystems. Demzufolge ist eine Verschiebung des Verhältnisses der in Abbildung 32 dargestellten möglichen bereinigten Gesamtnutzenergie zugunsten der bereinigten Nutzenergie der Umgebungsluft denkbar. Der Vergleich (Tab. 2) der bereinigten Gesamtnutzenergie (GNE) mit der bereinigten Kollektornutzenergie (KNE) hat gezeigt, dass im Mai und Juni eine negative Energiedifferenz (ED) auftritt. Diese negative Energiedifferenz kann wie folgt 
erklärt werden: Bei der bereinigten Kollektornutzenergie ist noch ein thermischer Energiegewinn erzielt worden. Die durch die Temperaturdifferenz gewonnene thermische Energie hat dagegen nicht ausgereicht, um die benötigte Ventilatorantriebsenergie zu kompensieren. Die geringere bereinigte Gesamtnutzenergie gegenüber der bereinigten Kollektornutzenergie ist dadurch zu erklären, dass neben der zuvor genannten Bedingung auch die Bedingung für das Ansaugen von Umgebungsluft nicht erfüllt wurde. Aufgrund der zu erwartenden Verringerung der Ventilatorantriebsenergie für das Ansaugen von Umgebungsluft ist in weiterführenden Untersuchungen zu klären, ab welcher Temperaturdifferenz es energetisch sinnvoller ist, die thermische Energie der Umgebungsluft zu nutzen. Es ist weiterhin zu prüfen, ob der zusätzliche Nutzen die Kosten für den technischen Aufwand rechtfertigt.

Ein ähnlicher Ansatz ist in der Literatur zu finden. Bereits Grimm (1984) hat anhand seiner Untersuchungen empfohlen, bei negativen Temperaturdifferenzen Umgebungsluft für einen Wärmepumpenbetrieb anzusaugen [34].

\subsubsection{Thermischer Energiebedarf der Jugendherberge}

Der in Abbildung 33 dargestellte thermische Energiebedarf beruht auf dem gemessenen Gesamtwärmebedarf und dem berechneten Energiebedarf für die Brauchwassererwärmung. Der abgebildete Heizungsbedarf in den Sommermonaten wird vornehmlich durch die Auskühlung der Brauchwasserzirkulationsleitung und des Brauchwasserpufferspeichers verursacht. Im Februar ist die Jugendherberge geschlossen.

Die für die Berechnung getroffenen Annahmen bei der verbrauchten Warmwassermenge und Warmwassertemperatur unterliegen einem stark nutzerabhängigen Verbraucherverhalten. Aus diesem Grund kann der berechnete Energiebedarf für die Brauchwassererwärmung nur als Annäherung an den tatsächlichen Bedarf betrachtet werden. Für die exakte Bestimmung ist eine kontinuierliche Erfassung des Warmwasserverbrauches 
mit einem Wärmemengenzähler notwendig, da auch das ankommende Brauchwasser einer Temperaturschwankung unterliegt. Neben der Ermittlung des Wärmebedarfes ist es ebenfalls von Vorteil, die Brauchwasserentnahmezeiten zu erfassen, um gegebenenfalls die Wärmepumpenlaufzeiten auf das Verbraucherverhalten abstimmen zu können.

\subsubsection{Simulation der Wärmetauschertemperaturen}

Für die Simulation der Wärmetauschertemperaturen wurde entschieden, das Jahr aufgrund der sich am Tagestemperaturverlauf orientierenden meteorologischen Jahreszeit aufzuteilen. Auf eine reine temperaturbasierte Aufteilung wurde verzichtet, da es das Ziel ist, jahreszeitabhängige Unterschiede herauszuarbeiten. Zum anderen geht durch diese Art der Aufteilung die Tageszugehörigkeit der Messwerte verloren. Ein Nachteil dieser Vorgehensweise ist, dass die im Praxisbetrieb zum Teil erheblichen Schwankungen der Lufttemperatur durch die Zusammenfassung nur noch indirekt für die einzelnen Monate berücksichtigt sind. Dies hat zur Folge, dass die mittlere Lufttemperatur über oder unter der tatsächlichen Temperatur der einzelnen Monate liegen kann und die tatsächlichen Gegebenheiten verzerrt. Hierdurch ist es möglich, dass bei der Simulation des Wärmepumpenbetriebes die abgegebene Wärmemenge über oder unter der theoretisch möglichen Wärmemenge liegt. Um diese monatsspezifischen Schwankungen besser herausarbeiten zu können, hätten für jeden Monat einzeln die Soletemperaturen berechnet werden müssen. Da die Wärmetauscherberechnungen nicht selbst durchgeführt werden konnten, musste dieser Kompromiss eingegangen werden. Neben den durch die gebildeten Mittelwerte verursachten Ungenauigkeiten muss bei der Interpretation der nachfolgenden Berechnungen bedacht werden, dass die Luftemperatur und sämtliche daraus resultierenden Wärmetauschertemperaturen auch im Tagesgang erheblichen Schwankungen unterliegen.

Bei der Betrachtung der berechneten Luftaustrittstemperaturen (LAT) der Tabelle 3 lässt sich vermuten, dass es mit dem vorhandenen Wärmetauscher 
nicht möglich ist, die Luft bis auf $10^{\circ} \mathrm{C}$ abzukühlen. Diese Vermutung wird dadurch bestärkt, dass der Wärmetauscher für einen doppelt so hohen Luftvolumenstrom und eine geringere Solespreizung ausgelegt ist. Demzufolge wird das durch den Solardach-Luft-Kollektor zur Verfügung gestellte Potenzial schlechter genutzt als in den Berechnungen zur thermischen Nutzenergie angenommen. Es ist zu erwarten, dass durch einen an die Bedingungen besser angepassten Wärmetauscher das Potenzial des Solardach-Luft-Kollektors effizienter genutzt werden kann. Durch eine angepasste Auslegung des Wärmetauschers wird weiterhin erwartet, dass auch Luftemperaturen unter $10^{\circ} \mathrm{C}$ von der Wärmepumpe genutzt werden können. Werden Soletemperaturen um den Gefrierpunkt genutzt, ist es denkbar, dass bei einer Vereisung des Wärmetauschers dieser durch die Zirkulation der erwärmten Kollektorluft bei abgeschalteter Wärmepumpe wieder aufgetaut werden kann. Folglich ist von einer positiven Auswirkung auf die Wärmepumpenlaufzeiten auszugehen.

Die besonders in den Sommermonaten erzielten hohen Lufttemperaturen werfen die Frage auf, ob es nicht sinnvoll sein kann, diese Lufttemperaturen direkt zur Brauchwasservorerwärmung ohne die Unterstützung der Wärmepumpe zu nutzen [60]. Um diese Frage zu klären, müssen in weiterführenden Untersuchungen der erzielbare Nutzen und der damit verbundene Aufwand bestimmt werden. Eine weitere Möglichkeit ist, die durch den Wärmetauscher abgekühlte Luft in den Sommermonaten zur Gebäudeklimatisierung zu nutzen. Es wird davon ausgegangen, dass durch diesen zusätzlichen Nutzen die Effizienz des Gesamtsystems gesteigert werden kann.

Der von Grimm (1984) beschriebene Nutzungszeitraum des Solar-LuftKollektors zur Brauchwassererwärmung wird vom 1. Mai bis 30. September angegeben [34]. Dieser Nutzungszeitraum unterscheidet sich von den eigenen Ergebnissen, bei denen auch im April und Oktober ein Beitrag zur Brauchwassererwärmung erzielt werden kann. Der Vergleich der Luftabkühlung durch den Wärmetauscher (LAT) zeigt, dass diese Abkühlung bei den Untersuchungen von Grimm (1984) deutlich geringer ausgefallen ist [34]. Es wird vermutet, dass die Ursachen für diese Abweichung auf die 
unterschiedlichen Dimensionierungen der Wärmetauscher und der Wärmepumpen zurückzuführen sind.

\subsubsection{Simulation des Wärmepumpenbetriebes}

\subsubsection{Vollständige Wärmeabnahme}

Bei der verwendeten Wärmepumpe standen keine nach DIN EN 14511 und DIN EN 255-3 ermittelten Leistungszahlen zur Verfügung $[17,18]$. Die Ermittlung der Leistungszahl wurde daher anhand des technischen Datenblattes des Verdichters durchgeführt [74]. Für die Berechnung wurde ein linearer Zusammenhang zwischen der Verdampfungsleistung und der elektrischen Antriebsleistung unterstellt. Dies entspricht jedoch nur näherungsweise dem realen Zusammenhang, ist jedoch aufgrund eines zu gering aufgelösten Verdichterkennfeldes nicht zu umgehen gewesen. Der für die Berechnung der Leistungszahl in der Formel (14) einzusetzende Anlagenfaktor $\left(a_{f}\right)$ wurde von dem Wärmepumpenhersteller mit 0,99 angegeben und deshalb vernachlässigt [65]. Es gilt jedoch zu prüfen, ob dieser Anlagenfaktor nicht durch Abstrahlverluste niedriger anzusetzen ist [45].

Nach der DIN EN 15450 sind die verschiedenen möglichen Systemgrenzen für die Berechnung der Jahresarbeitszahl definiert [16]. Für die Berechnung wurde sich jedoch an die Vorgaben der VDI Richtlinie 4650 gehalten. Die in der VDI Richtlinie 4650 vorgesehenen Korrekturfaktoren für die Berechnung der Jahresarbeitszahl konnten zum einen aufgrund fehlender Informationen zum Verdichter und zum anderen aufgrund eingeschränkter Zuordnungsmöglichkeiten bei den Betriebsbedingungen nicht berücksichtigt werden [71]. Das von dem Bundesverband Wärmepumpe e. V. (BWP) kostenlos zur Verfügung gestellte Online-Programm zur Berechnung der Jahresarbeitszahl nach VDI Richtlinie 4650 ist aufgrund der verwendeten und nicht aufgeführten Wärmepumpe mit drei separaten Heizkreisläufen nicht anwendbar gewesen [11]. Durch die Ermittlung der Arbeitszahlen für die 
unterschiedlichen Jahreszeiten sind die jahreszeitabhängigen Wärmequellenschwankungen annähernd berücksichtigt worden. Bei der Berücksichtigung der Hilfsenergien wurde für die bedarfsgesteuerte Solepumpe die maximale Leistungsaufnahme angerechnet. Es ist jedoch zu bedenken, dass diese Leistungsaufnahme bei hohen Soletemperaturen auch niedriger ausfallen kann. Unberücksichtigt blieb, dass möglicherweise die Motorabwärme der Solepumpe die Soletemperatur zusätzlich erhöht. Ebenfalls unberücksichtigt blieb die Leistungsaufnahme während der Bereitschaftsperiode. Weiterhin ist zu bedenken, dass bei der Berechnung der Arbeitszahl nur ein Verdichter betrachtet wurde. Schaltet sich bei einem ausreichenden Wärmeangebot der zweite Verdichter ein, wird mit dem gleichen Pumpenund Ventilatoraufwand ein höherer Nutzen erzielt. Das bedeutet, dass sich bei dem Betrieb beider Verdichter die Hilfsenergien jeweils zur Hälfte auf die Verdichter aufteilen und die Arbeitszahl steigt. Eine von vornherein anzurechnende Halbierung der Hilfsenergien wurde aufgrund einer Bevorteilung bei einem nicht ausreichenden Wärmeangebot verworfen. Ab welchem Wärmeangebot sich der zweite Verdichter zuschaltet und wie sich dies auf die Arbeitszahl auswirkt, muss in weiterführenden Untersuchungen geklärt werden. Die angewendete Berechnungsmethode ist eine sehr vereinfachte Form zur Ermittlung der Jahresarbeitszahl und entspricht nur annähernd dem vorgeschriebenen Verfahren. Aufgrund der nicht berücksichtigbaren Korrekturfaktoren ist zu erwarten, dass besonders durch die zum Teil erheblich schwankende Wärmequelle die ermittelte Jahresarbeitszahl im Praxisbetrieb sinkt [46, 71]. Aus den beschriebenen Gründen ist es notwendig, die Jahresarbeitszahl in weiterführenden Untersuchungen anhand der abgegebenen Nutzwärme und der eingesetzten elektrischen Energie für den Antrieb des Verdichters und der Hilfsantriebe im laufenden Betrieb zu bestimmen.

Anhand der Ergebnisse der berechneten Leistungszahlen des Verdichters aus Tabelle 4 kann geschlussfolgert werden, dass die Temperaturniveaus der Wärmequelle (Jahreszeiten) und der warmen Seite der Wärmepumpe einen entscheidenden Einfluss auf die Leistungszahl haben. Dieser 
grundlegende Zusammenhang wurde bereits im Literaturteil erläutert und bestätigt die eigenen Ergebnisse [31,62]. Der Vergleich der Leistungszahlen $\left(\varepsilon_{\mathrm{V}}\right)$ aus Tabelle 4 mit den Arbeitszahlen $(\beta)$ aus Tabelle 5 lässt ebenfalls die Schlussfolgerung zu, dass neben den beschriebenen Einflussfaktoren auf die Leistungszahl die elektrische Antriebsenergie der Hilfsaggregate die Höhe der Arbeitszahl entscheidend beeinflusst. Die Ergebnisse aus Tabelle 6 zeigen, dass selbst bei der niedrigsten Pufferspeichertemperatur lediglich eine Arbeitszahl von 3,11 erzielt wird. Für die Einstufung als effiziente Wärmepumpe sind nach dem ErneuerbareEnergien-Wärmegesetz (EEWärmeG) Mindest-Jahresarbeitszahlen für elektrisch angetriebene Wärmepumpen gefordert. Bei Neubauten ist die geforderte Mindest-Jahresarbeitszahl für Luft/Wasser Wärmepumpen 3,5 und für alle anderen Wärmepumpen 4,0 [6]. Diese Mindest-Jahresarbeitszahl sollte als unterer Richtwert für die Pilotanlage angesehen werden. Ziel muss es sein, die Arbeitszahl gegenüber einer mit reiner Umgebungsluft betriebenen Luft/Wasser Wärmepumpe deutlich anzuheben, um den zusätzlichen Aufwand für die Luftvorerwärmung zu rechtfertigen. Die Einordnung der verwendeten Sole/Wasser Wärmepumpe in die Nomenklatur des EEWärmeG ist schwierig, da dieser Wärmepumpentyp normalerweise die Erdwärme und nicht Luft als Wärmequelle nutzt [6,62]. Es ist jedoch offensichtlich, dass bereits die ohne Korrekturfaktoren ermittelte Jahresarbeitszahl nicht den Anforderungen eines effizienten Wärmepumpenbetriebes nach dem EEWärmeG entspricht [5]. Dies liegt zum größten Teil daran, dass durch die Verwendung eines Zwischenwärmeträgermediums nicht unerhebliche Wärmeverluste entstehen. Weiterhin ist die niedrige Jahresarbeitszahl dem Umstand geschuldet, dass durch bauliche Gegebenheiten ein zweigeteiltes System verwendet werden musste. Durch die räumliche Trennung von Wärmetauscher und Wärmepumpe wird ein höherer Pumpenaufwand als bei einer Kompaktbauweise verursacht. Hinzu kommt, dass aufgrund der vorgeschriebenen hohen Brauchwassertemperatur eine Wärmepumpe mit Heißgasabschöpfung eingesetzt wurde. Hierzu wurden zwei separate Heizkreisläufe für eine stufenweise Anhebung der Brauch- 
wassertemperatur installiert. Weiterhin wurde ein dritter Heizkreislauf zur Heizungsunterstützung eingesetzt, um möglichst lange Wärmepumpenlaufzeiten zu erzielen. Dadurch wurden im Vergleich zu einer herkömmlichen Wärmepumpe zwei zusätzliche Umwälzpumpen benötigt, die sich ebenfalls negativ auf die Jahresarbeitszahl auswirken. Um die Effizienz der Pufferspeicher der drei Heizkreisläufe beurteilen zu können, ist es neben den Wärmemengenzählern der Wärmepumpe notwendig, die Pufferspeichertemperaturen und die Temperaturen der ein- und austretenden Wassermenge sowie die Volumenströme zu bestimmen. Bei der Brauchwasservorerwärmung wird erwartet, dass in dem Pufferspeicher mit der niedrigsten Temperatur eine höhere Brauchwassererwärmung als in dem nachgelagerten Pufferspeicher erzielt wird. Die für alle Berechnungen getroffene Annahme, dass das Heizungssystem die für die Heizungsunterstützung bereitgestellte Energie vollständig nutzt, gilt es zu prüfen. Die in diesem Berechnungsmodell angenommene vollständige Wärmeabnahme wird in den meisten Anwendungsfällen nicht erreicht. Der Grund ist die Divergenz zwischen dem solaren Wärmeangebot und der Wärmenachfrage. Im Sommer ist das solare Wärmeangebot hoch und die Wärmenachfrage gering. Bei einem geringeren solaren Wärmeangebot steigt dagegen die Wärmenachfrage im Winter.

In der Literatur konnte bereits durch die Untersuchungen von Lücke (1984) gezeigt werden, dass es möglich ist, mit der Unterstützung eines Solar-LuftKollektors die Leistungszahl einer Luft/Wasser Wärmepumpe anzuheben. Ein Vergleich der Leistungszahlen ist jedoch aufgrund der unterschiedlichen Betriebsbedingungen nicht möglich. Eine Ermittlung der Arbeitszahl wurde in diesen Untersuchungen nicht durchgeführt [56]. 


\subsubsection{Wärmeabnahme in der Jugendherberge}

Die Berechnung der Wärmeabnahme beruht auf den Annahmen des zuvor diskutierten thermischen Energiebedarfes für die Brauchwassererwärmung und unterliegt deshalb dem gleichen nutzerabhängigen Verbraucherverhalten. Das angenommene erzielbare Temperaturniveau der Brauchwasservorerwärmung ist abhängig von der Differenz zwischen der Verdichtungsendtemperatur der Wärmepumpe und der Heizungspufferspeichertemperatur. Je kleiner die Temperaturdifferenz ist, desto höher ist die zur Verfügung stehende Temperatur in den beiden Pufferspeichern zur Brauchwasservorerwärmung. Die Höhe der Verdichtungsendtemperatur ist von der Soletemperatur abhängig. Bei einer niedrigen Soletemperatur steigt die Verdichtungsendtemperatur. Steigt dagegen die Soletemperatur an, sinkt die Verdichtungsendtemperatur. Diese Beziehung resultiert aus der vom Verdichter zu überwindenden Differenz zwischen dem Verflüssigungs- und Verdampfungsdruck. Je niedriger die zu überwindende Druckdifferenz ist, desto weniger Verdichterarbeit muss, bezogen auf die Verdampferleistung, verrichtet werden und desto niedriger ist die Verdichtungsendtemperatur [4, 63]. Die Höhe des Temperaturniveaus im Heizungspufferspeicher ist von dem Rücklauftemperaturniveau der konventionellen Heizungsanlage abhängig. Das Rücklauftemperaturniveau ist im Sommer aufgrund der einzuhaltenden Brauchwassertemperaturen nahezu konstant auf einem höheren Niveau. Mit dem Beginn des Raumheizungsbedarfes in der Übergangszeit sinkt das Temperaturniveau und steigt nur bedarfsweise für die Brauchwassererwärmung. Der dargestellte Zusammenhang zeigt, dass die als konstant angenommene Temperatur der Brauchwasservorerwärmung während des Wärmepumpenbetriebes Schwankungen unterliegt und von mehreren Parametern abhängt. Aus diesem Grund ist die berechnete Wärmeabnahme der Jugendherberge als ein Näherungswert zu betrachten. Neben diesen Parametern kommt hinzu, dass die Anzahl der Übernachtungen nicht für alle Tage in einem Monat gleich ist, sondern fluktuiert. Dies hat zur Folge, dass es zu einem ungünstigeren Verhältnis zwischen der möglichen abgegebenen Wärmemenge und der 
Wärmeabnahme der Jugendherberge kommen kann. Durch diese Divergenz kann sich die berechnete Wärmepumpenlaufzeit verringern. Für die genaue Bestimmung ist, wie bereits zuvor bei dem thermischen Energiebedarf für die Brauchwassererwärmung diskutiert, ein Wärmemengenzähler einzubauen und neben der entnommenen Wärmemenge sind die Brauchwasserentnahmezeiten zu registrieren. Es gilt weiterhin zu erwähnen, dass nach dem DVGW-Arbeitsblatt W 551 (DVGW, Deutscher Vereinigung des Gasund Wasserfaches e.V.) auch die Pufferspeicher zur Brauchwasservorerwärmung einmal am Tag auf mindestens $60^{\circ} \mathrm{C}$ zu erwärmen sind [20]. Es handelt sich zwar um Pufferspeicher, die mit Heizungswasser beladen sind, diese werden jedoch zur Brauchwasservorerwärmung mit Brauchwasser durchflossen. Bei einer sehr geringen Brauchwasserabnahme kann es daher vorkommen, dass das bereits vorerwärmte Brauchwasser für einen längeren Zeitraum in den Pufferspeichern zum Stehen kommt. Da die Legionellen besonders in dem zu erwartenden Temperaturbereich der Brauchwasservorerwärmung zwischen $30^{\circ} \mathrm{C}$ und $45^{\circ} \mathrm{C}$ wachsen, ist diese technische Regel einzuhalten [20].

Die bei dieser Betrachtung (Tab. 8) berechneten Wärmepumpenlaufzeiten (WLZ) beziehen sich wiederum auf den Betrieb von nur einem Verdichter. Schaltet sich bei einem ausreichenden Wärmeangebot der zweite Verdichter ein, erhöht sich die abgegebene Wärmemenge. Aus diesem Grund ist zu erwarten, dass sich die negativen Wärmebedarfsdeckungen (WBD) im laufenden Betrieb zum Teil verringern. Weiterhin ist es möglich, dass sich durch die höhere abgegebene Wärmemenge die Wärmepumpenlaufzeit (WLZ) aufgrund einer mangeInden Wärmeabnahme verkürzt. Durch das Zuschalten des zweiten Verdichters halbieren sich die Hilfsenergien und demzufolge steigt die Arbeitszahl, wie bereits zuvor diskutiert. Die geringfügige Verschlechterung der Jahresarbeitszahl aus Tabelle 10 gegenüber der zu erzielenden Jahresarbeitszahl bei einer vollständigen Wärmeabnahme aus Tabelle 6 ist anhand der geringeren Wärmeabnahmen in den Monaten August und September zu begründen. Durch diese geringeren Wärmeabnahmen verschieben sich die prozentualen Anteile im 
Jahresverlauf und die Jahresarbeitszahl sinkt geringfügig. Eine Anhebung der Arbeitszahl ist durch die Vorgabe eines Zeitfensters für den Wärmepumpenbetrieb bei einer nicht vollständigen Wärmeabnahme zu erzielen. Dieses Zeitfenster muss so gelegt sein, dass ein Wärmepumpenbetrieb nur bei höheren Lufttemperaturen stattfindet. Das Zeitfenster muss so groß sein, dass die Wärmeabnahme der Jugendherberge gedeckt ist. Die diskutierten Einflussfaktoren zeigen, dass es notwendig ist, die zu erzielende Jahresarbeitszahl in weiterführende Untersuchungen anhand der abgegebenen Wärmemenge und der aufgenommenen elektrischen Antriebsenergie im laufenden Betrieb zu ermitteln.

\subsubsection{Optimierung}

Bei den Berechnungen eines optimierten Wärmepumpenbetriebes wurde der Solekreislauf durch den Kältekreislauf der Wärmepumpe (Direktverdampfung) ersetzt. Es wurde unterstellt, dass durch die Druckdifferenz hinter dem Expansionsventil auf eine Wärmequellenpumpe verzichtet werden kann. Die räumliche Trennung der Wärmepumpe und des Wärmetauschers hat jedoch zur Folge, dass der Verdichter einen höheren Strömungswiderstand überwinden muss [71]. Durch den höheren Strömungswiderstand im Rohrsystem ist es denkbar, dass der Verdichter eine etwas höhere elektrische Antriebsenergie benötigt. Die mit $5 \mathrm{~K}$ angenommene höhere Verdampfungstemperatur des Kältemittels erscheint durch den Wegfall eines mit Wärmeverlusten behafteten Wärmetauschers realisierbar [45]. Es ist jedoch anzumerken, dass für den Kältekreislauf im Gegensatz zum Solekreislauf polierte Rohrleitungen und ein spezieller Wärmetauscher notwendig sind. Weiterhin wurde unterstellt, dass durch eine Optimierung des Rohrsystems zur Luftabsaugung mindestens $15 \%$ der Ventilatorantriebsenergie eingespart werden kann. Diese Überlegung beruht auf der Grundlage der zuvor bei der Luftabsaugung diskutierten Reduktion des Strömungswiderstandes. Es ist weiterhin zu erwarten, dass die Höhe der Reduktion unter Umständen größer ausfallen kann. Liegt die zu überwindende 
Gesamtdruckdifferenz nicht wesentlich höher als $350 \mathrm{~Pa}$, ist es möglich, den identischen Luftvolumenstrom mit nur einem Ventilator zu fördern [29]. Die für die Optimierung getroffenen Annahmen sind anhand weiterer Untersuchungen zu verifizieren. Im Weiteren unterliegt der optimierte Wärmepumpenbetrieb den gleichen Gegebenheiten, die zuvor diskutiert wurden.

Die Ergebnisse aus Tabelle 13 zeigen, dass es durch den Wegfall des Solekreislaufes möglich zu sein scheint, nicht nur die nach dem EEWärmeG geforderten Mindest-Jahresarbeitszahl für Luft/Wasser Wärmepumpen zu erreichen, sondern auch, die für die übrigen Wärmepumpen geltende Jahresarbeitszahl von 4,0 zu überschreiten [6]. Die berechneten Jahresarbeitszahlen aus Tabelle 13 zeigen, dass durch die Optimierung die Jahresarbeitszahlen von über 4,0 bis zu einem Temperaturniveau von $40^{\circ} \mathrm{C}$ auf der warmen Seite bei einer vollständigen Wärmeabnahme realisierbar zu sein scheinen. Es ist davon auszugehen, dass auch bei der Wärmeabnahme der Jugendherberge Jahresarbeitszahlen über 4,0 erzielt werden können. Der Grund für die mögliche Abnahme der Jahresarbeitszahl in der Jugendherberge wurde zuvor anhand der geringeren Wärmepumpenlaufzeiten diskutiert. Diese vereinfachten Simulationsrechnungen sollen eine erste Einschätzung geben. Die tatsächlich erzielbare Jahresarbeitszahl ist in einem Praxisversuch zu bestimmen.

Im Folgenden werden weitere Möglichkeiten einer Jahresarbeitszahlerhöhung durch Senkung der Hilfsenergien diskutiert. Es ist möglich, dass die Beladung der Pufferspeicher mit einer Schwerkraftzirkulation erfolgt. Hierdurch können die drei Heizungsumwälzpumpen mit einer Anschlussleistung von je $75 \mathrm{~W}$ eingespart werden. Der Nachteil dieser Beladung ist, dass diese ständig aktiv ist und bei einer abgeschalteten Wärmepumpe durch den rückwärtigen Schwerkraftumlauf Wärmeverluste verursacht werden. Diese Wärmeverluste können durch den Einbau einer Schwerkraftumlaufsperre verhindert werden [63,69]. Nach dem DVGW-Arbeitsblatt W 551 sind jedoch Schwerkraftzirkulationen aus hygienischer Sicht bei der Trinkwassererwärmung nicht geeignet [20]. Aus diesem Grund ist nur die 
Umwälzpumpe des Heizungspufferspeichers durch eine Schwerkraftzirkulation zu ersetzen. In Abbildung 32 wurde gezeigt, dass es unter bestimmten Bedingungen Sinn macht, Umgebungsluft über einen Bypass anzusaugen. Dadurch ist zu erwarten, dass sich die zu überwindende Gesamtdruckdifferenz verringert und die Ventilatorantriebsenergie sinkt. Anhand dieser Einschätzung ist zu prüfen, ob es nicht im Sommer aufgrund der ohnehin hohen Lufttemperaturen und der Divergenz zwischen Globalstrahlungsangebot und Wärmenachfrage in der Summe effektiver sein kann, in den Mittagsstunden Umgebungsluft anzusaugen. Der Grund für die zu erwartende geringere Druckdifferenz wurde bereits bei der energetischen Betrachtung des Daches diskutiert.

Eine weitere Möglichkeit bietet sich durch das Ansaugen der erwärmten Dachraumluft. In der Abbildung 17 und Abbildung 18 wurde gezeigt, dass die Dachraumtemperatur über der Umgebungstemperatur liegt. Diese positive Temperaturdifferenz gibt Anlass, über die Nutzung der Dachraumluft nachzudenken. In weiteren Untersuchungen muss jedoch zunächst geklärt werden, wie hoch die zu entziehende thermische Energie ist und welche zu überwindende Gesamtdruckdifferenz sich durch die Nutzung der Dachraumluft einstellt. Es wird erwartet, dass diese Druckdifferenz entscheidend von der über die Firstlüftung nachströmenden Luftmenge abhängt. Sollten die Untersuchungen ergeben, dass das thermische Energieangebot der Dachraumluft über einen angemessenen Zeitraum genutzt werden kann, ist es denkbar, zusätzliche Lüftungsklappen für ein möglichst hindernisfreies Nachströmen der Umgebungsluft vorzusehen. Demzufolge wird neben dem zusätzlichen Wärmegewinn eine geringere Ventilatorantriebsenergie gegenüber dem Kollektorbetrieb erwartet.

Bei den relativ niedrigen Luftgeschwindigkeiten im Solardach-Luft-Kollektor stellt sich die Frage, ob es nicht möglich ist, auf eine Ventilatorunterstützung zu verzichten und die natürliche Thermik des Solardach-Luft-Kollektors zu nutzen. Die erwärmte Luft steigt auf der Sonnenseite nach oben, wird durch den in Firsthöhe installierten Wärmetauscher abgekühlt und sinkt auf der sonnenabgewandten Dachfläche wieder nach unten. Über einen am First zu 
installierenden Bypass könnte bei einer negativen Temperaturdifferenz die Umgebungsluft als Wärmequelle genutzt werden. Neben der technischen Realisierbarkeit muss zunächst in weiterführenden Untersuchungen geklärt werden, ob der durch die natürliche Thermik entstehende Luftvolumenstrom und die realisierbare Temperaturerhöhung für einen effektiven Wärmetauscherbetrieb ausreichend sind. Dazu müssen zunächst die erforderliche Höhe des Strömungskanals, die Dachlänge und Dachneigung sowie die erforderliche Dachfläche bestimmt werden. Es wird vermutet, dass im Vergleich zu einem ventilatorunterstützten Betrieb ein geringerer Luftvolumenstrom gefördert wird und dadurch eine erheblich größere Dachfläche erforderlich ist. Die hier diskutierten Möglichkeiten für einen optimierten Wärmepumpenbetrieb in Kombination mit einem Solardach-Luft-Kollektor zeigen, dass weiterer Forschungsbedarf besteht.

\subsection{5 Ökonomische Betrachtung}

\subsubsection{Investitionskosten der solaren Energiegewinnung}

Der Tabelle 14 kann entnommen werden, dass die größte Kostenposition durch die Wärmepumpentechnik und die Pufferspeicher verursacht wurde. Dies liegt zum einen daran, dass trotz eines zu hohen Temperaturniveaus in der Jugendherberge versucht wurde, einen Wärmepumpenbetrieb zu realisieren. Aus diesem Grund wurden eine mit höheren Kosten verbundene Wärmepumpe mit Heißgasabschöpfung und zwei zusätzliche Pufferspeicher benötigt. Für die Pufferspeicher zur Brauchwasservorerwärmung sind aufgrund einer notwendigen Spezialanfertigung erhebliche Kosten entstanden. Zum anderen sind durch die räumliche Trennung der Wärmepumpe und des Wärmetauschers weitere Kosten verursacht worden. Durch die Bindung an einen Generalunternehmer sind für die Installationsarbeiten der Stauwärmenutzungsanlage ebenfalls höhere Kosten entstanden. Die im Verhältnis geringen Investitionskosten für den Solardach-Luft-Kollektor liegen darin begründet, dass nur Kosten, die über den Investitionskosten für die 
eigentliche Dacheindeckung lagen, berücksichtigt wurden. Es ist weiterhin zu erwähnen, dass es Marktanreizprogramme zur Förderung von effizienten Wärmepumpen und Solarkollektoren auf der Grundlage des EEWärmeG gibt [6]. Nicht abgedeckte Solarkollektoren sind jedoch nach den Richtlinien zur Förderung von Maßnahmen zur Nutzung erneuerbarer Energien im Wärmemarkt von einer Förderung ausgeschlossen [7, 8]. Diese Fördermöglichkeiten wurden zum einen aufgrund des Versuchsanlagenstatuses nicht in Anspruch genommen und zum anderen werden in dieser Untersuchung ausschließlich die Kosten für die Bereitstellung der solaren Energiegewinnung betrachtet.

\subsubsection{Energiebezugskosten und Amortisationszeitraum}

Für die Berechnung der Energiebezugskosten der Wärmepumpe wurde der mittlere Strompreis aus dem Haupt- und Nebentarif der Jugendherberge angenommen. Dies ist jedoch nur zutreffend, wenn unterstellt wird, dass die Wärmepumpe in den Haupt- und Nebentarifzeiten zu gleichen Anteilen betrieben wird. Es ist zu erwarten, dass sich der Anteil der Nebentarifzeit zugunsten der Haupttarifzeit aufgrund der niedrigeren Luftemperaturen in der Nacht verschiebt. Die Beantragung eines ebenfalls tageszeitabhängigen Wärmepumpenstromtarifes wurde aufgrund eines zu hohen Aufwandes und einer im Verhältnis zu geringen Kosteneinsparung nicht realisiert [48]. Bei der Berechnung der Energiebezugskosten für das konventionelle Heizungssystem der Jugendherberge wurde der Norm-Nutzungsgrad des GasBrennwertkessels zugrunde gelegt. Dieser unter Prüfbedingungen ermittelte Nutzungsgrad wurde aufgrund der Vergleichbarkeit mit der unter annähernd standardisierten Bedingungen ermittelten Jahresarbeitszahl ausgewählt [14]. Es ist zu erwarten, dass im Praxisbetrieb neben der Jahresarbeitszahl auch der Norm-Nutzungsgrad des Gas-Brennwertkessels sinkt. Diese beiden Parameter haben neben dem aktuellen Strom- und Gaspreis einen entscheidenden Einfluss auf die Energiebezugskosten. Um den im Praxisbetrieb erzielbaren Nutzungsgrad des Gas-Brennwertkessels bestimmen zu 
können, ist es daher notwendig, neben der abgegebenen Wärmemenge auch den Gasverbrauch zu registrieren.

Für die Berechnung des Amortisationszeitraumes wurde unterstellt, dass das konventionelle Heizungssystem auch ohne die solare Energiegewinnung benötigt wird, aus diesem Grund wurden nur die Investitionskosten für die solare Energiegewinnung angesetzt. Es ist darauf hinzuweisen, dass bei einer Wirtschaftlichkeitsbetrachtung die üblichen ökonomischen Kostenansätze zu berücksichtigen sind. Unberücksichtigt blieben auch die um 8-12\% höher anzusetzenden Investitionskosten für die polierten Rohrleitungen und den speziellen Wärmetauscher bei der Direktverdampfung. Aus den bereits bei den Investitionskosten der solaren Energiegewinnung diskutierten Gründen sind die Investitionskosten als überproportional hoch einzuordnen. Infolgedessen wurden für eine erste Einschätzung des Systems die ermittelten Investitionskosten als maximale Investitionskosten für alle Betriebsmodelle angesetzt.

Die berechneten Energiebezugskosten haben gezeigt, dass eine hohe Arbeitszahl und eine möglichst lange Wärmepumpenlaufzeit (WLZ) bei einer hohen Wärmeabnahme sowie ein möglichst geringes Temperaturniveau auf der warmen Seite der Wärmepumpe die eingesparten Energiebezugskosten durch die Wärmepumpe steigern. Zwischen der zu erzielenden maximalen Arbeitszahl und einer möglichst langen Wärmepumpenlaufzeit besteht jedoch ein Zielkonflikt. Die Untersuchungen haben gezeigt: Je höher die zur Verfügung stehenden Lufttemperaturen sind, desto höher ist die zu erzielende Arbeitszahl der Wärmepumpe. Durch eine Nutzungseinschränkung kann die Arbeitszahl bei einer schwankenden Wärmequelle erhöht werden, im Gegensatz verringert sich jedoch dadurch die mögliche Wärmepumpenlaufzeit. In weiterführenden Untersuchungen ist zu klären, bei welchem Verhältnis zwischen Arbeitszahl und Wärmepumpenlaufzeit der größte ökonomische Nutzen erzielt werden kann. Ziel muss es sein, das Wärmeangebot möglichst optimal über einen längeren Zeitraum zu nutzen. 
Bei der Berechnung der Amortisation hat sich, wie zu erwarten, gezeigt, dass sich die überdurchschnittlich hohen Investitionskosten in der Jugendherberge negativ auf den Amortisationszeitraum der bestehenden Anlage auswirken. Diese Berechnung bezieht sich wiederum auf den Betrieb mit nur einem Verdichter. Wird unterstellt, dass das Wärmeangebot im gesamten Betrachtungszeitraum für den Betrieb beider Verdichter ausreichend ist, können sich die eingesparten Energiebezugskosten bei einer vollständigen Wärmeabnahme maximal verdoppeln und so den Amortisationszeitraum bestenfalls halbieren. Auch diese Annahme offenbart, dass ein ökonomisch sinnvoller Betrieb mit der bestehenden Stauwärmenutzungsanlage nicht möglich ist (vgl. Tab. 17). Diese hohen Investitionskosten können zum Teil durch die, bei einer Optimierung des Wärmepumpenbetriebes zu erwartende, höhere Einsparung bei den Energiebezugskosten mit einer angenommenen vollständigen Wärmeabnahme kompensiert werden. Um den Amortisationszeitraum auf einen annähernd vertretbaren Zeitraum zu senken, müssten sich die Investitionskosten, neben der Optimierung des Wärmepumpenbetriebes, mindestens halbieren. Das Verhältnis zwischen den Investitionskosten und den eingesparten Energiebezugskosten der untersuchten Anlage macht deutlich, dass die bestehende Anlage bereits ohne zusätzliche Kostenansätze nicht wirtschaftlich ist.

Der Vergleich mit der Literatur zeigt, dass die bei einem optimierten Wärmepumpenbetrieb zu erwartende Einsparung von rund $52 \%$ gegenüber den Energiebezugskosten des konventionellen Heizungssystems deutlich höher ausfällt. Grimm (1984) beschreibt in seinen Untersuchungen, dass die Energiekosten um 1/3 der konventionellen Ölheizung reduziert werden können. Diese geringere Einsparung ist hauptsächlich damit zu begründen, dass Grimm (1984) in seinen Berechnungen neben den Energiebezugskosten auch eine Abschreibung für die solare Brauchwassererwärmung berücksichtigt hat [34]. Die unterschiedliche ökonomische Beurteilung der annähernd vergleichbaren Systeme ist anhand der erheblich höheren Investitionskosten in der Jugendherberge zu begründen. 


\subsection{Praktische Umsetzung}

In den Untersuchungen hat sich herausgestellt, dass eine der zentralen Fragestellungen die richtige Einbindung der durch die Wärmepumpe bereitgestellten Energie in das Heizungssystem der Jugendherberge ist. Das Ziel ist es, die Wärmepumpe an der Stelle mit dem niedrigsten Temperaturniveau in das vorhandene Heizungssystem einzubinden. Je geringer die Temperaturdifferenz zwischen der Wärmequelle und dem benötigten Temperaturniveau ist, desto besser ist die Leistungszahl $\left(\varepsilon_{\mathrm{V}}\right)$ bzw. die Arbeitszahl ( $\beta$ ) (vgl. Tab. 5 und Tab. 12) [31, 62]. Wärmepumpen eignen sich aus diesem Grund besonders für Vorlauftemperaturen von bis zu $35^{\circ} \mathrm{C}$ $[48,62]$. Die eingebaute Wärmepumpe kann zwar aufgrund einer Heißgasabschöpfung höhere Temperaturen zur Verfügung stellen, die beschriebenen grundlegenden Zusammenhänge gelten jedoch auch für diese Wärmepumpentechnik. Dies bedeutet für den Wärmepumpenbetrieb in der Jugendherberge, dass nur bei einer ausreichenden Wärmeabnahme durch die Wärmesenke hohe Temperaturen zur Verfügung gestellt werden können. Technisch ist es zwar möglich, mit der verwendeten Wärmepumpe höhere Vorlauftemperaturen zu realisieren, dies geht jedoch unweigerlich zu Lasten der Arbeitszahl ( $\beta$ ) (vgl. Tab. 5 und Tab. 12).

Die geforderte Mindest-Jahresarbeitszahl nach dem EEWärmeG kann zwar aller Voraussicht nach durch die Optimierung des Wärmepumpenbetriebes erreicht werden, ob ein ökonomisch sinnvoller Betrieb möglich ist, muss sich jedoch in weiteren Untersuchungen zeigen.

Für einen ökonomisch sinnvollen Betrieb muss es das Ziel sein, aufwändige und kostenintensive Lösungen zu vermeiden. Weiterhin sollte die Wärmepumpennutzungsanlage möglichst geringe Vorlauftemperaturen besitzen und eine kontinuierliche Wärmeabnahme gewährleisten. Die tageszeitabhängigen Schwankungen der Wärmequelle müssen durch einen Pufferspeicher ausgeglichen werden. Aufgrund des schwankenden Wärmeangebotes in den unterschiedlichen Jahreszeiten eignet sich das System zur Unterstützung eines saisonal unabhängigen Heizungssystems. 
Bevor die Stauwärmenutzungsanlage in der Praxis angewendet werden kann, ist das Optimum zwischen dem technisch Machbaren und ökonomisch Sinnvollen in weiterführenden Untersuchungen zu bestimmen. 


\section{$6 \quad$ Zusammenfassung}

Bereits in den 80er Jahren wurde von Grimm (1984) und Lücke (1984) nachgewiesen, dass mit handelsüblichen Metallprofilen eingedeckte Dachflächen in Verbindung mit einer Wärmepumpe zur Brauchwassererwärmung geeignet sind. Die aktuellen Entwicklungen auf dem Energiemarkt gaben Anlass, dieses kostengünstige Konzept der Stauwärmenutzung erneut aufzugreifen und einem Praxistest zu unterziehen. Zur Praxiserprobung wurde der Neubau der Jugendherberge des Deutschen Jugendherbergswerkes in Dahme (Ostsee) mit einem Prototyp des Solardaches mit Metalleindeckung ausgestattet. Das Solardach entspricht systematisch einem Solardach-LuftKollektor ohne Abdeckung (Absorber), der in der Jugendherberge in Verbindung mit einer Wärmepumpe zur Brauchwasservorerwärmung und Heizungsunterstützung eingesetzt wird. Ziel der vorliegenden Untersuchung war es, das Betriebsverhalten der bestehenden Pilotanlage zu bestimmen. Da die jetzt bestehende Pilotanlage in mehreren Entwicklungsstufen entstanden ist und der gegenwärtige Zustand der Anlage noch keine belastbare Auswertung der Stauwärmenutzungsanlage zulässt, wurden zunächst Untersuchungen an dem Solardach-Luft-Kollektor durchgeführt und im Anschluss diese Ergebnisse in Form von Simulationsrechnungen auf das dahinter stehende Nutzungssystem bezogen.

Die aufgestellte Hypothese, dass Dachflächen, die mit handelsüblichen Metalldachprofilen eingedeckt sind, sich zur thermischen Energiegewinnung eignen, konnte durch die Untersuchungen bestätigt werden. Die durch das Solardach erzielte Anhebung der Kollektoreintrittsluft liegt mit bis zu $24 \mathrm{~K}$ im für Solar-Luft-Kollektoren typischen Bereich. Dagegen liegt der erzielte maximale Wirkungsgrad aufgrund des geringen geförderten Luftvolumenstromes, bezogen auf die Kollektorfläche, nur knapp über $6 \%$. Dieser geringe Wirkungsgrad kann jedoch in Kauf genommen werden, da es das Ziel ist, einen möglichst hohen Temperaturanstieg der Kollektoreintrittsluft durch die kostengünstige Nutzung des Daches zu erzielen. 
Die Berechnungen haben gezeigt, dass sich das Solardach in Verbindung mit einer Wärmepumpe zur Brauchwassererwärmung und Heizungsunterstützung eignet. Es konnte nachgewiesen werden, dass bei einer Wärmepumpe mit Direktverdampfung die geforderte Mindest-Jahresarbeitszahl nach dem Erneuerbare-Energien-Wärmegesetz (EEWärmeG) erreicht werden kann.

Eine ökonomische Betrachtung der Stauwärmenutzungsanlage hat gezeigt, dass es möglich ist, die Energiebezugskosten der Jugendherberge zu senken. Durch das hohe Temperaturniveau der konventionellen Heizungsanlage wurden jedoch erhebliche zusätzliche Investitionskosten verursacht. Ein ökonomisch sinnvoller Betrieb der bestehenden Stauwärmenutzungsanlage ist aufgrund dieser Gegebenheit in der Jugendherberge nicht realisierbar.

Es ist jedoch zu erwarten, dass mit einem Heizungssystem, das für einen Wärmepumpenbetrieb geeignet ist, die Investitionskosten erheblich reduziert werden können und ein ökonomisch sinnvoller Betrieb möglich ist. Diese Untersuchungen bestätigen die Aussagen von Grimm(1984) und Lücke (1984), dass sich mit handelsüblichen Metalldachprofilen eingedeckte Dachflächen in Verbindung mit einer Wärmepumpe zur Brauchwassererwärmung eignen. Die Untersuchungen haben aber auch gezeigt, dass für die praktische Umsetzung noch weiterer Forschungsbedarf besteht. In weiterführenden Untersuchungen ist zu klären, wie der Solardach-LuftKollektor technisch und ökonomisch sinnvoll auf einen möglichst effizienten Wärmepumpenbetrieb abzustimmen ist. 


\section{$7 \quad$ Ausblick}

Zukünftig ist es denkbar, die Energiedachlösung insbesondere bei landwirtschaftlichen Gebäuden einzusetzen, da diese Gebäude häufig über große Dachflächen verfügen. Neben der indirekten Nutzung des Solardach-LuftKollektors zur Brauwassererwärmung und Heizungsunterstützung bietet sich hier eine direkte Nutzung der erwärmten Luft bei landwirtschaftlichen Trocknungsanlagen an. Durch den Einsatz der kostengünstigen Energiedachlösung ist es möglich, den Anteil an Fremdenergie zu reduzieren und somit die Betriebskosten der Anlage deutlich zu senken. Im Gegensatz zu den klassischen Heizsystemen ist die Trocknungsluft prinzipbedingt frei von Verbrennungsrückständen. Ein weiterer Vorteil ist der Wegfall der verlustbehafteten thermischen Umwandlung, wie sie bei herkömmlichen Trocknungsverfahren vorhanden ist. Das Solardach eignet sich im besonderen Maße für Trocknungsanlagen mit minimiertem Energieeinsatz. Bei leistungsmaximierten Trocknungsanlagen können die Betriebskosten durch den Einsatz von vorgewärmter Luft gesenkt werden.

Ein zusätzlicher Effekt der Stauwärmenutzung ist die Kühlung des Gebäudes an heißen Sommertagen. Es wird vermutet, dass sich die Nutzung der heißen Dachluft positiv auf das Gebäudeklima auswirkt. Denkbar wäre auch die direkte Nutzung der vorgewärmten Luft zur Regulierung des Stallklimas, z. B. in Ferkelaufzuchtställen.

Es scheint jedoch unabdingbar, dass der Solardach-Luft-Kollektor auf den jeweiligen Verwendungszweck und das notwendige Temperaturniveau sowohl technisch als auch ökonomisch zu optimieren ist. 


\section{$8 \quad$ Literaturverzeichnis}

[1] Arulanandam, S. J., Hollands, K. G. T. und Brundrett, E. (1999): A CFD heat transfer analysis of the transpired solar collector under no-wind conditions. Solar Energy, 67, S. 93-100.

[2] Belusko, M.; Saman, W. und Bruno, F. (2004): Roof integrated solar heating system with glazed collector. Solar Energy, 76, S. 61-69.

[3] Bonnet, C. und Storch, A. (2006): Einfluss der Himmelstemperatur auf abgedeckte und unabgedeckte Kollektoren. http://www.arsenal.ac.at/ downloads/Publikationen/2005/Der\%20Einfluss\%20der\%20Himmels temperatur.pdf, gesehen am 05.02.2010.

[4] Bukau, F. (1983): Wärmepumpen-Technik. Oldenbourg Verlag GmbH, München [u.a.], S. 55-57.

[5] Bundesgesetzblatt (2001): Verordnung über die Qualität von Wasser für den menschlichen Gebrauch (Trinkwasserverordnung - TrinkwV 2001). Bundesgesetzblatt Teil I vom 28.05.2007, Nr. 24, Bonn, S. 959980.

[6] Bundesgesetzblatt (2008): Gesetz zur Förderung Erneuerbarer Energien im Wärmebereich (Erneuerbare-Energien-Wärmegesetz EEWärmeG). Bundesgesetzblatt Teil I vom 18.08.2008, Nr. 36, Bonn, S. 1658-1665.

[7] Bundesministerium für Umwelt Naturschutz und Reaktorsicherheit (2009): Richtlinien zur Förderung von Maßnahmen zur Nutzung erneuerbarer Energien im Wärmemarkt. Bundesministerium für Umwelt, Naturschutz und Reaktorsicherheit, Berlin.

[8] Bundesministerium für Umwelt Naturschutz und Reaktorsicherheit (2010): Änderung der Richtlinien zur Förderung von Maßnahmen zur Nutzung erneuerbarer Energien im Wärmemarkt. Bundesministerium für Umwelt, Naturschutz und Reaktorsicherheit, Berlin. 
[9] Bundesministerium für Wirtschaft und Technologie (BMWi) (2009): Energie in Deutschland - Trends und Hintergründe zur Energieversorgung in Deutschland. http://www.bmwi.de/Dateien/Energieportal/PDF/ energie-in-deutschland, property $=$ pdf, bereich=bmwi, sprache $=\mathrm{de}, \mathrm{rwb}=$ true.pdf, gesehen am 08.04.2010.

[10] Bundesverband Wärmepumpe (BWP) e. V. (2009): BWPBranchenstudie 2009 - Szenarien und politische Handlungsempfehlungen. Bundesverband Wärmepumpe (BWP) e. V., Berlin.

[11] Bundesverband Wärmepumpe e. V. (2009): JAZ-Rechner. http://www. waermepumpe.de/fachpartner/jaz-rechner.html, gesehen am 18.02.2010.

[12] Cattin, R. (2008): Alpine Test Site Gütsch Handbuch und Fachtagung, Schlussbericht. http://www.bfe.admin.ch/php/modules/enet/streamfile. php?file $=000000010091$.pdf\&name $=000000280211$, gesehen am 10.02.2010.

[13] Conserval Engineering Inc. (2009): Dachaufbau eines SolarWall Luftkollektors. http://solarwall.de/html/dachmontage.html, gesehen am 12.01.2009.

[14] DIN Deutsches Institut für Normung e. V. (1990): DIN 4702-8, Heizkessel; Ermittlung des Norm-Nutzungsgrades und des NormEmissionsfaktors. DIN Deutsches Institut für Normung e. V., Berlin.

[15] DIN Deutsches Institut für Normung e. V. (2003): DIN EN ISO 5167-3, Durchflussmessung von Fluiden mit Drosselgeräten in voll durchströmten Leitungen mit Kreisquerschnitt - Teil 3: Düsen und Venturidüsen (ISO 5167-3:2003); Deutsche Fassung EN ISO 51673:2003. DIN Deutsches Institut für Normung e. V., Berlin.

[16] DIN Deutsches Institut für Normung e. V. (2007): DIN EN 15450, Heizungsanlagen in Gebäuden - Planung von Heizungsanlagen mit Wärmepumpen; Deutsche Fassung EN 15450:2007. DIN Deutsches Institut für Normung e. V., Berlin. 
[17] DIN Deutsches Institut für Normung e. V. (2008): DIN EN 255-3 (Entwurf), Luftkonditionierer, Flüssigkeitskühlsätze und Wärmepumpen mit elektrisch angetriebenen Verdichtern - Heizen - Prüfungen und Anforderungen an die Kennzeichnung von Geräten zum Erwärmen von Brauchwarmwasser; Deutsche Fassung prEN 25. DIN Deutsches Institut für Normung e. V., Berlin.

[18] DIN Deutsches Institut für Normung e. V. (2009): DIN EN 14511-3 (Entwurf), Luftkonditionierer, Flüssigkeitsküh/sätze und Wärmepumpen mit elektrisch angetriebenen Verdichtern für die Raumbeheizung und Kühlung - Teil 3: Prüfverfahren; Deutsche Fassung prEN 14511-3:2009. DIN Deutsches Institut für Normung e. V., Berlin.

[19] DVGW Deutsche Vereinigung des Gas- und Wasserfaches e. V. (1998): DVGW Arbeitsblatt W 553, Bemessung von Zirkulationssystemen in zentralen Trinkwassererwärmungsanlagen. DVGW Deutsche Vereinigung des Gas- und Wasserfaches e. V., Bonn.

[20] DVGW Deutsche Vereinigung des Gas- und Wasserfaches e. V. (2004): DVGW Arbeitsblatt W 551, Trinkwasserenwärmungs- und Trinkwasserleitungsanlagen - Technische Maßnahmen zur Verminderung des Legionellenwachstums - Planung, Errichtung, Betrieb und Sanierung von Trinkwasser-Installationen. DVGW Deutsche Vereinigung des Gas- und Wasserfaches e. V., Bonn.

[21] Danfoss GmbH (2009): Wärmepumpenprospekt DHP-H Opti Pro. http://www.at.waermepumpen.danfoss.com/PCMPDF/Buntprospekt\%2 0DHP-H\%200pti\%20Pro_10\%202009.pdf, gesehen am 10.04.2010.

[22] Deans, J. und Weerakoon, A. (2008): The thermal performance of a solar air heater. 5th European Thermal-Sciences Conference, The Netherlands.

[23] Debiel, B. (2010): E-Mail. Meteomedia GmbH, Bochum, 19.04.2010.

[24] Deutsche Energie-Agentur GmbH (2007): Infoblätter Lufttechnik: Planung und Optimierung luftechnischer Anlagen. http://www.industrieenergieeffizienz.de/uploads/media/L08-Planung-Optimierung_01.pdf, gesehen am 04.04.2010. 
[25] Duffie, J. A. und Beckman, W. A. (2006): Solar Engineering of Thermal Processes. John Wiley \& Sons, Inc., Hoboken, S. 148-149, 164-166, 282-292.

[26] Eicker, U. (2001): Solare Technologien für Gebäude. B. G. Teubner $\mathrm{GmbH}$, Stuttgart [u.a.], S. 118-120, 136-138.

[27] Fachinformationszentrum Karlsruhe (2002): Solare Luftsysteme. FIZ, Eggenstein-Leopoldshafen.

[28] Filleux, C.; Gütermann, A. und Primas, A. (2005): Solare Luftheizsysteme. Ökobuch Verlag, Staufen bei Freiburg.

[29] Fischbach Luft- und Ventilatorentechnik GmbH (2004): Datenblatt Compact-Gebläse CE 670/E 25. Fischbach Luft- und Ventilatorentechnik $\mathrm{GmbH}$, Neunkirchen.

[30] Fischer, K. (2005): Taschenbuch der technischen Formeln. Fachbuchverlag Leipzig im Carl Hanser Verlag, München [u.a.], S. 273.

[31] Flade, F. in Zusammenarbeit mit dem Bundesverband Wärmepumpe (BWP) e. V. (2008): Arbeitsordner Wärmepumpe. Marketing + Wirtschaft Verlagsgesellschaft Flade \& Partner mbH, München, S. 10.

[32] Fördergesellschaft Technischer Ausbau e. V. (1981): FTAFachberichte - Wärmepumpen zur Hausheizung. Technischer Verlag Resch KG, Gräfelfing, S. 45-46.

[33] Glück, B. (1991): Zustands- und Stoffwerte. Verlag für Bauwesen, Berlin, S. 12, 49.

[34] Grimm, W. (1984): Anwendung, Aufbau und Betriebscharakteristika von Solardach-Luft-Kollektoren in der Landwirtschaft. MEG-Schrift 71, Dissertation Universität Göttingen.

[35] Grimm, W. (1984): Grundlagen zur Berechnung und Dimensionierung von großflächigen Sonnenkollektoren zur Lufterwärmung. Grundlagen der Landtechnik, 34, Nr. 1, S. 25-32. 
[36] Gunnewiek L. H.; Brundrett E.; Hollands K. G. T. (1996): Flow distribution in unglazed transpired plate solar air heaters of large area. Solar Energy, 58, S. 221-237.

[37] Gunnewiek, L. H.; Hollands, K. G. T. und Brundrett, E. (2002): Effect of wind on flow distribution in unglazed transpired-plate collectors. Solar Energy, 72, S. 317-325.

[38] Hartmann, W. (1979): Trocknung von Getreide und Halmfutter mit solarerwärmter Luft in Satztrocknungsanlagen. MEG-Schrift 34, Dissertation Universität Göttingen, S. 74-75, 93-95.

[39] Hastings, S. R. und Mørck, O. (2000): Solar air systems. James und James, London.

[40] Heidler, K.; Hindenburg, C.; Kasper, B.; Metz, M.; Quaschning, V.; Schnauss, D.; Spitzmüller, P. und Weyres-Borchert, B. (2008): Solarthermische Anlagen. DGS, Deutsche Gesellschaft für Sonnenenergie e. V. Landesverband Berlin Brandenburg e. V. und Landesverband Hamburg/Schleswig-Holstein e. V., Berlin.

[41] ITEC Entwicklungs- und Vertriebsgesellschaft mbH (2007): KönigHochtemperatur-Topladetechnik. http://www.koenig-waermepumpen. de/koenig_waermepumpen/index.pl?PLUG=BasicSite\&act=SP\&PAGE =58, gesehen am 10.04.2010.

[42] Keller, J. und Kyburz, V. (1987): Dimensionierung von Sonnenkollektoren für die Heubelüftung. Eidgenössische Forschungsanstalt für Betriebswirtschaft und Landtechnik (FAT), Tänikon.

[43] Khartchenko, N. V. (1995): Thermische Solaranlagen - Grundlagen, Planung und Auslegung. Springer-Verlag, Berlin [u.a.], S. 34-48, 50.

[44] Khartchenko, N. V. (2004): Thermische Solaranlagen. Verlag für Wissenschaft und Forschung $\mathrm{GmbH}$, Berlin, S. 5-8, 47-48, 70-73.

[45] Kirn, H. (1983): Grundlagen der Wärmepumpentechnik. C. F. Müller $\mathrm{GmbH}$, Karlsruhe, S. 1-84, 198-204. 
[46] Kirn, H. (1983): Wärmequellen und Wärmespeicher. C. F. Müller $\mathrm{GmbH}$, Karlsruhe, S. 83.

[47] Kirn, H. und Hadenfeldt, A. (1976): Wärmepumpen. C. F. Müller $\mathrm{GmbH}$, Karlsruhe, S. 1-13, 24-25, 76, 96.

[48] Kirn, H. und Hadenfeldt, A. (1987): Anwendung der Elektrowärmepumpe. C. F. Müller $\mathrm{GmbH}$, Karlsruhe, S. 21-35, 124-129, 148-151, 169-171, 191-195.

[49] Korschelt, D. und Lackmann, J. (1995): Lehr- und Übungsbuch Strömungsmechanik. Fachbuchverlag Leipzig $\mathrm{GmbH}$, Leipzig, S. 37-38.

[50] Kutscher, C. F. (1994): Heat exchanger effectiveness and pressure drop for air flow through perforated plated with and without crosswind. Journal of Heat Transfer, 116, S. 391-399.

[52] Kutscher, C. F., Christensen, C. B. und Barker, G. M. (1993): Unglazed Transpired Solar Collectors: Heat Loss Theory. Journal of Solar Energy, 115, S. 182-188.

[52] Ladener, H. und Späte, F. (2003): Solaranlagen - Handbuch der thermischen Solarenergienutzung. Ökobuch Verlag, Staufen bei Freiburg, S. 27-31, 60-62.

[53] Lenzen, M. (2007): Schriftliche Mitteilung. Arthur Grillo GmbH, Ratingen, 11.07.2007.

[54] Lindab GmbH (2004): Dimensionieren von Rohrsystemen. http://www.lindab.de/dokumenter/Dimensionieren\%20von\%20Rohr. pdf, gesehen am 02.05.2008.

[55] Lufttechnik Schmeißer GmbH (2010): LTS Wärmepumpe mit Heißgasabschöpfung für Trinkwarmwasser. http://www.lufttechnikschmeisser .de/waermepumpen.html, gesehen am 10.04.2010. 
[56] Lücke, W. (1984): Vergleichende Hallen- und Freilandversuche an Solardach-Kollektoren. MEG-Schrift 108, Dissertation Universität Göttingen.

[57] Lücke, W. und Hörsten, D. v. (2006): Stauwärmenutzung in Dächern Energiegewinnung in landwirtschaftlichen Gebäuden. Landtechnik, 61, Nr. 4, S. 208-209.

[58] Meyer, G. und Schiffner, E. (1989): Technische Thermodynamik. VCH Verlagsgesellschaft mbH, Weinheim, S. 189.

[59] Mitsubishi Electric Europe B. V. (2009): Wärmepumpen-Broschüre. http://www.mitsubishi-electric-aircon.de/pdf/Waermepumpeninfo_36 Seiten.pdf, gesehen am 10.04.2010.

[60] Mørck, O. (2000): Air-to-water heat exchangers. In: S. R. Hastings und O. Mørck (Hrsg.) Solar air systems, James \& James, London, S. 212-214.

[61] Mørck, O. und Fechner, H. (2000): Flat-plate air collectors. In: S. R. Hastings und O. Mørck (Hrsg.) Solar air systems, James \& James, London, S. 133-145.

[62] Ochsner, K. (2005): Wärmepumpen in der Heizungstechnik. C. F. Müller Verlag, Heidelberg, S. 9-12, 18-19, 41.

[63] Recknagel, H.; Sprenger, E. und Schramek, R. (2008): Taschenbuch für Heizung + Klimatechnik 09/10 - auf DVD. Oldenbourg Industrieverlag, München, S. 208-209, 691, 711-714, 970, 1621, 1921, 1953-1957.

[64] Sandmann, A. (2010): Telefonische Mitteilung. Jugendherberge Dahme, Dahme, 06.01.2010.

[65] Santore, K. (2010): Telefonische Mitteilung. Karl Santore GmbH, Neuendorf, 12.08.2009.

[66] Santore, S. (2010): E-Mail. Karl Santore GmbH, Neuendorf, 14.01.2010. 
[67] Schmidt, M.; Richter, W.; Kolarik, F. und Lampert, J. (2005): Bestimmung des Energiebedarfs zur Optimierung von Luftkanalsystemen Raumlufttechnischer Anlagen Teil II. http://www.ige.unistuttgart.de/ fileadmin/ressourcenRedakteure/pdf/Berichte//Bolka2.pdf, gesehen am 29.03.2010.

[68] Schrader, R. (2009): Telefonische Mitteilung. Adolf Thies GmbH \& Co. KG, Göttingen, 07.05.2009.

[69] Schreier, N.; Wagner, A.; Orths, R. und Rotarius, T. (2002): Solarwärme optimal nutzen. Wagner \& Co Solartechnik $\mathrm{GmbH}$, Cölbe, S. 61-62.

[70] Stiebel Eltron GmbH \& Co. KG (2010): Wasser und Wärme im grünen Bereich. http://www.stiebel-eltron.de/imperia/md/content/STIEBELELT RON/de/Fachpartner/Fachinformationen/Prospekte/Bestellung/ee_bro schuere_2010_kl_de.pdf, gesehen am 10.04.2010.

[71] VDI Verein Deutscher Ingenieure e. V. (2008): VDI 4650 Blatt 1, (Entwurf) Berechnungen von Wärmepumpen - Kurzverfahren zur Berechnung der Jahresarbeitszahl von Wärmepumpenanlagen Elektro-Wärmepumpen zur Raumheizung und Warmwasserbereitung. Verein Deutscher Ingenieure e. $V$., Düsseldorf.

[72] VDI Verein Deutscher Ingenieure e. V. - Fachbereich Technische Gebäudeausrüstung (2000): VDI 2067 Blatt 12, Wirtschaftlichkeit gebäudetechnischer Anlagen - Nutzenergiebedarf für die Trinkwassererwärmung. Verein Deutscher Ingenieure e. V., Düsseldorf.

[73] Van Decker, G. W. E.; Hollands, K. G. T. und Brunger, A. P. (2001): Heat exchange relations for unglazed transpired solar collectors with circular holes on a square or triangular pitch. Solar Energy, 71, S. 33-45.

[74] Viertel, J. (2010): Faxmitteilung. Wätas Wärmetauscher Sachsen $\mathrm{GmbH}$, Olbernhau, 16.02.2010.

[75] Viessmann Werke GmbH \& Co KG (2009): Datenblatt Victocrossal 300 Typ CT3, Gas-Brennwertkessel für Erdgas E, LL und Flüssiggas. Viessmann Werke GmbH \& Co KG, Allendorf (Eder). 
[76] Wieneke, F.; Lücke, W. und Grimm, W. (1984): Abschlussbericht zum DFG-Forschungsvorhaben Wi 208/50, Erfassung des Betriebsverhaltens von einfachen Solardach-Luft-Kollektoren für die landwirtschaftliche Trocknung und Warmwasserbereitung. Institut für Agrartechnik der Georg-August-Universität Göttingen, Göttingen. 


\section{Lebenslauf}

\begin{tabular}{|c|c|}
\hline \multicolumn{2}{|l|}{ Angaben zur Person } \\
\hline Nachname, Vorname & Baum, Matthias \\
\hline Staatsangehörigkeit & Deutsch \\
\hline Geburtsdatum und -ort & 10. Mai 1977 in Eschwege \\
\hline Geschlecht & Männlich \\
\hline \multicolumn{2}{|l|}{ Berufserfahrung } \\
\hline Zeitraum & Seit 2010 \\
\hline Beruf oder Funktion & Manager Erneuerbare Energien \\
\hline Name des Arbeitgebers & $\mathrm{R}+\mathrm{V}$ Allgemeine Versicherung AG \\
\hline Zeitraum & 2010 \\
\hline Beruf oder Funktion & Wissenschaftlicher Mitarbeiter \\
\hline Name des Arbeitgebers & $\begin{array}{l}\text { Technische Universität Clausthal, } \\
\text { Energie-Forschungszentrum Niedersachsen }\end{array}$ \\
\hline Zeitraum & $2006-2010$ \\
\hline Beruf oder Funktion & Wissenschaftlicher Mitarbeiter \\
\hline Name des Arbeitgebers & $\begin{array}{l}\text { Georg-August-Universität Göttingen, Department für } \\
\text { Nutzpflanzenwissenschaften, Abteilung Agrartechnik }\end{array}$ \\
\hline Zeitraum & $1996-2000$ \\
\hline Beruf oder Funktion & $\begin{array}{l}\text { Angestellter, } \\
\text { ab } 1999 \text { Deckstellenleiter }\end{array}$ \\
\hline Name des Arbeitgebers & Niedersächsisches Landgestüt Celle \\
\hline \multicolumn{2}{|l|}{$\begin{array}{r}\text { Schul- und } \\
\text { Berufsausbildung }\end{array}$} \\
\hline Zeitraum & $2001-2006$ \\
\hline $\begin{array}{r}\text { Bezeichnung der erworbenen } \\
\text { Qualifikation }\end{array}$ & Master of Science \\
\hline Fachrichtung & Wirtschafts- und Sozialwissenschaften des Landbaus \\
\hline $\begin{array}{l}\text { Name und Art der } \\
\text { Bildungseinrichtung }\end{array}$ & $\begin{array}{l}\text { Fakultät für Agrarwissenschaften der Georg-August- } \\
\text { Universität Göttingen }\end{array}$ \\
\hline Zeitraum & 2001 \\
\hline Art der Ausbildung & Wehrdienst in Fritzlar \\
\hline
\end{tabular}




\begin{tabular}{|c|c|}
\hline Zeitraum & 2000 \\
\hline $\begin{array}{r}\text { Bezeichnung der erworbenen } \\
\text { Qualifikation }\end{array}$ & $\begin{array}{l}\text { Pferdewirtschaftsmeister Schwerpunkt: } \\
\text { Zucht und Haltung }\end{array}$ \\
\hline Zeitraum & 1997 \\
\hline $\begin{array}{r}\text { Bezeichnung der erworbenen } \\
\text { Qualifikation }\end{array}$ & Besamungsbeauftragter für Pferde \\
\hline Zeitraum & $1993-1996$ \\
\hline $\begin{array}{r}\text { Bezeichnung der erworbenen } \\
\text { Qualifikation }\end{array}$ & Pferdewirt Schwerpunkt: Zucht und Haltung \\
\hline $\begin{array}{r}\text { Name und Art der } \\
\text { Bildungseinrichtung }\end{array}$ & Hessisches Landgestüt Dillenburg \\
\hline Zeitraum & $1987-1993$ \\
\hline $\begin{array}{r}\text { Bezeichnung der erworbenen } \\
\text { Qualifikation }\end{array}$ & Mittlere Reife \\
\hline $\begin{array}{r}\text { Name und Art der } \\
\text { Bildungseinrichtung }\end{array}$ & Brüder-Grimm-Schule in Eschwege \\
\hline Zeitraum & $1983-1987$ \\
\hline $\begin{array}{l}\text { Name und Art der } \\
\text { Bildungseinrichtung }\end{array}$ & Grundschule in Eschwege \\
\hline
\end{tabular}




\section{Danksagung}

An dieser Stelle möchte ich mich bei folgenden Personen bedanken, die mich bei der Anfertigung meiner Dissertation unterstützt haben.

Meinem Doktorvater, Herrn Prof. Dr. Wolfgang Lücke, gilt mein besonderer Dank für die Anregung zu dieser Arbeit und das entgegengebrachte Vertrauen.

Für ihre ständige Diskussionsbereitschaft und die Übernahme des Koreferates bedanke ich mich herzlich bei Frau PD Dr. Engel Hessel.

Herrn Dr. Dieter von Hörsten danke ich für seine wertvolle Kritik und Hilfsbereitschaft.

Ein großes Dankeschön geht an die Mitarbeiterinnen und Mitarbeiter der Abteilung Agrartechnik sowie die Familie Santore.

Bei Frau Sabine Kliebisch und Frau Christiane Kunze bedanke ich mich recht herzlich für die vielen Stunden Korrekturlesen.

Ganz besonders herzlich bedanke ich mich bei meinen Eltern und meiner Freundin Juliane, die mich jederzeit unterstützt und mir den Rücken gestärkt haben.

Mein Dank gilt weiterhin der Deutschen Bundesstiftung Umwelt für die finanzielle Förderung des Forschungsprojektes Stauwärmenutzung zur Brauchwassererwärmung mittels Metalldach (Solar-Luft-Kollektor Jugendherberge Dahmeshövd), ohne dieses Forschungsprojekt wäre die vorliegende Arbeit nicht möglich gewesen.

Ebenfalls bedanke ich mich bei dem DJH Landesverband Nordmark e. V. für die Aufgeschlossenheit gegenüber dem Forschungsprojekt und die gute Zusammenarbeit.

Herrn Jörg Kachelmann und dem Team der Meteomedia GmbH danke ich für die zur Verfügung gestellten Wetterdaten.

Ferner bedanke ich mich bei allen hier nicht namentlich aufgeführten Personen, die zum Gelingen dieser Arbeit beigetragen haben. 